\title{
Propriedades estruturais e elásticas de fases lamelares: \\ O efeito da composição da membrana
}

\section{Barbara Bianca Gerbelli}

Orientadora: Profa. Dra. Elisabeth Andreoli de Oliveira

Dissertação apresentada ao Instituto de Física da Universidade de São Paulo para a obtenção do título de Mestre em Ciências.

Comissão Examinadora:

Profa. Dra. Elisabeth Andreoli de Oliveira (Orientadora, IFUSP)

Profa. Dra.Carla Goldman (IFUSP)

Prof. Dr.Amando Siuiti Ito (FFCLRP)

São Paulo 
FICHA CATALOGRÁFICA

Preparada pelo Serviço de Biblioteca e Informação do Instituto de Física da Universidade de São Paulo

Gerbelli, Barbara Bianca

Propriedades estruturais e elásticas de fases lamelares: 0 efeito da composição da membrana. São Paulo, 2012.

Dissertação (Mestrado) - Universidade de São Paulo. Instituto de Física - Depto. de Física Experimental

Orientador: Profa. Dra. Elisabeth Andreoli de Oliveira

Área de Concentração: Biofísica experimental

Unitermos: 1.Física da Materia Condensada; 2. Biofísica estatística; 3.Mudança de fase.

USP/IF/SBI-001/2013 
A minha família... 

Ao men querido Pedro. 

"A tarefa não é tanto ver aquilo que nínguem viu, mas pensar o que ninguém ainda pensou sobre aquilo que todo mundo vê".

Arthur Schopenhauer 



\section{Agradecimentos}

Primeiramente eu agradeço a Deus por estar ao meu lado em todos os instantes, começando desde as pessoas que fui encontrando na minha jornada como minha família, médicos, grandes mestre que me inspiraram para o que sou hoje.

A minha família por aguentar todas as minhas reclamações, estresses e entre outros causos. Agradeço a minha mãe por não ser somente o meu braço esquerdo, mas sim muitas vezes ser o meu braço direito e esquerdo. A você mãe, agradeço pela mulher que sou, aos meus irmãos que me fizeram aprender o que é família e esta ser prioridade para mim. Ao meu pai pelo dom da vida e Denise por todos os conselhos. A todos os meus tios, mas principalmente ao meu tio Ganso que sempre me disse "Bá! Nunca podemos parar de estudar!”; tio cá estou nunca parando!

Agradeço a uma nova família que entrou na minha vida para ficar e agradeço à todos da família Oseliero que junto desta pertence o meu “namorido” Pedro, que agradeço por toda paciência durante nossa história e saiba que sou muito grata a você, parte deste trabalho só se concretizou porque você estava ao meu lado.

Agradeço a Elisabeth pela chance que você me deu desde o início da Iniciação Científica, obrigada por ser uma ótima orientadora, mas o que é mais importante uma grande mãe/amiga companheira desses últimos 6 anos que trabalhamos juntas.

Agradeço aos Professores Cristiano de Oliveira e Fréderic Nallet e a pesquisadora Laurence Navailles por toda a colaboração desde discussões, palpites e até mesmo criar um “software" para que eu pudesse analisar as amostras.

Agradeço aos meus amigos de sala e vizinha Renata, Rafael, Emerson, Vivian, Eraldo, JoernI, Cassio, Bruno, Sandra, Carlos mão no mouse, Daniel, Messias e Fernando que deixaram muitas estórias da convivência do dia a dia e congressos que vou poder sempre

Barbara Bianca Gerbelli - Propriedades estruturais e elásticas de fases lamelares: O efeito da composição da membrana. 
lembrar com muito carinho.

Agradeço ainda o Rafael e o Emerson, por me cederem dados para completar o meu trabalho, além de podermos discutir juntos nossos trabalhos que acredito que isso tenha enriquecido muito o meu mestrado.

Agradeço aos meus amigos de graduação Tassiana, Renata, Mauricio, Texas, Mary entre outros que me acompanharam em todo o árduo percurso da Graduação : festas, conversas, estudos, estudos e estudos.

Tem aqueles amigos que não são da turma dos doidos do IF que tenho que agradecer por toda a paciência quando eu sumia para ir para os laboratórios, congressos e até mesmo escrever a minha dissertação: Joice, Edneuma, Juju, Danilo, Japa, Juliana (GFCx), Carlinha, Livia e Carolzinha obrigada pela compreensão .

Agradeço a todos os técnicos que me ajudaram de alguma forma construir o meu aparato experimental: Odair, Zé Botina, Daniel e Igino. E claro o Serginho, Tarsis e Antonio Carlos da Cristalografia. Agradeço ao Grupo de Fluídos Complexos que me acompanham desde o início da minha Iniciação Científica e sempre ouvem "Uma molécula anfifílica é...”.

Agradeço a Universidade de São Paulo, aos funcionários da secretaria da Pós graduação que me ajudaram muito. Ao INCT de Fluidos Complexos, CAPES e FAPESP pelo auxilio financeiro.

Obrigada a todos que fizeram e fazem parte da minha vida, pois graças a vocês sou o que sou!

Barbara.

Barbara Bianca Gerbelli - Propriedades estruturais e elásticas de fases lamelares: O efeito da composição da membrana. 
Barbara Bianca Gerbelli - Propriedades estruturais e elásticas de fases lamelares: O efeito da composição da membrana. 


\section{RESUMO}

Neste trabalho, é apresentado um estudo sistemático de uma fase lamelar composta

de lecitina á qual é incorporado um co-surfactante de uso comercial (Simulsol ${ }^{\circledR}$ ), que consiste de uma mistura de ácidos graxos etoxilados. Foi realizado um estudo estrutural, utilizando a técnica de espalhamento de raios X em baixo ângulo (SAXS) em amostras inseridas em capilares, variando a composição da membrana, desde 100\% de lecitina até 100\% de Simulsol, ao longo da linha de diluição, para cada composição. Os resultados experimentais de espalhamento foram analisados usando um método que permite o ajuste direto da intensidade de espalhamento e um procedimento de otimização que fornece o perfil de densidade eletrônica da bicamada. Com esse método de análise, além de parâmetros estruturais tais como, periodicidade lamelar e espessura da bicamada obtém-se o parâmetro de Caillé, que relaciona as constantes elásticas que caracterizam a flexibilidade do sistema lamelar. As propriedades termodinâmicas do sistema lamelar foram investigadas para duas composições de membrana, em experimentos onde o potencial químico da água é controlado. A combinação dos resultados obtidos nos estudos estruturais e termodinâmicos permitiu a determinação do módulo de compressibilidade da fase lamelar e da constante de rigidez da membrana. A incorporação do co-surfactante aumenta a contribuição de interações de natureza entrópica relacionada às flutuações da membrana, que resultam na estabilização da fase lamelar para distâncias de separação entre as membranas muito maiores do que as observadas no sistema lamelar composto apenas por lecitina. Para pequenas distâncias de separação entre as bicamadas, observa-se superposição de regiões polares de bicamadas vizinhas, que são acompanhadas de mudanças no comportamento do parâmetro de Caillé, e consequentemente no comportamento das constantes de compressibilidade e na constante de rigidez. No regime diluído, a incorporação de moléculas anfifílicas com apenas uma cadeia carbônica resulta na redução significativa da constante de rigidez da membrana de lecitina de $\approx 20 k_{B} T$ para $\approx 0,5 k_{B} T$.

Barbara Bianca Gerbelli - Propriedades estruturais e elásticas de fases lamelares: O efeito da composição da membrana. 


\begin{abstract}
In this work we present a systematic study of structural properties of a lamelar phase composed of lecithin when a commercial cosurfactant $\left(\operatorname{Simulsol}{ }^{\circledR}\right)$ which is a mixture of etoxylated fatty acids. The study was carried out using small angle $\mathrm{X}$ ray scattering with samples encapsulated in glass capillaries, varying membrane composition from $100 \%$ of lecithin to $100 \%$ of Simulsol, through the dilution line, for each composition. The experimental results were analyzed using a method that fits directly the scattered intensity and, by means of an optimization procedure, gives the electronic density profile of the lipid bilayer. From the analysis of experimental data, in addition to the structural parameters, such as the lamellar period and membrane thickness, we obtain the Caillè parameter, which relates the elastic constants that characterizes the flexibility of the lamellar system. Thermodynamic properties of the lamellar system were also investigated, for two membrane compositions, by controlling the water chemical potential. The combinationof results obtained in structural and thermodynamic studies allowed determination of the compressibility modulus of the lamellar phase and the membrane rigidity constant. The incorporation of co-surfactant to the membrane increases the contribution of entropic interactions arising from membrane fluctuations resulting in the stabilization of lamellar phase for larger membrane separation distances than observed for the lamellar system composed of lecithin only. For small membrane separation distances, a superposition of polar region of neighbor bilayers is observed, with related changes in the behavior of Caillè parameter, and consequently in the behavior of compressibility and membrane rigidity constants. In diluted regime, the incorporation of amphiphilic molecules with a single carbonic chain results in a significant reduction in lecithin membrane rigidity, from $\approx 20 k_{B} T$ to $\approx 0,5 k_{B} T$.
\end{abstract}

Barbara Bianca Gerbelli - Propriedades estruturais e elásticas de fases lamelares: O efeito da composição da membrana. 

1. Introdução

I. . Motivação ........................... s

I.2. Estado da arte e objetivos . . . . . . . . . . . . 7

I.3. Organização . . . . . . . . . . . . . . . . . . 9

2. Fundamentação teórica $\quad 11$

2. I. Fenômenos de auto-organização . . . . . . . . . . . . I I

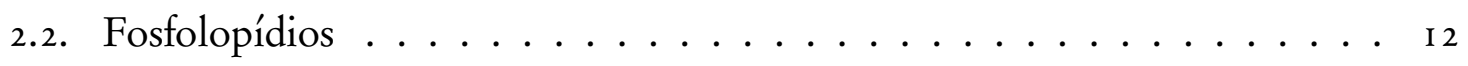

2.3. Fases Lamelares liotrópicas . . . . . . . . . . . . . . . . I 3

2.4. Interações entre bicamadas em fases lamelares . . . . . . . . . . . I4

2.5. Teoria de espalhamento de raios $\mathrm{X}$ em fases lamelares . . . . . . . . . I 8

2.5.I. Fator de estrutura e parâmetro de Caillé . . . . . . . . I 9

2.5.2. Fator de forma ................ 2 I

2.5.3. Propriedades elásticas das fases lamelares . . . . . . . . . 23

Barbara Bianca Gerbelli - Propriedades estruturais e elásticas de fases lamelares: O efeito da composição da membrana. 
3. Materiais e Métodos 29

3. . Preparação das Amostras . . . . . . . . . . . . . . . . . . . . 29

3.ı.ı. Composição das amostras . . . . . . . . . . . . . . . 29

3.I.2. Processo de preparação e estabilização das amostras . . . . . . . 29

3.2. Experimentos de SAXS e WAXS ................ 3 I

3.2.I. Fontes convencionais - Nanostar . . . . . . . . . 3 I

3.3. Métodos de tratamento e ajustes das curvas experimentais de SAXS $\quad \ldots \quad$. 32

3.3. I. Tratamento dos dados ................... 32

3.3.2. Ajuste das curvas de espalhamento . . . . . . . . . 35

3.3.3. Determinação da espessura da bicamada . . . . . . . . . . . 38

3.4. Experimentos de GISAXS ...................... 40

4. Resultados 45

4. I. Estudo Estrutural . . . . . . . . . . . . . . . . . 45

4. I. I. Membranas de lecitina .............. 45

4.I.2. Variação na composição da membrana . . . . . . . . . . . 57

4.I.3. Estudo termodinâmico ............... 7 I

$\begin{array}{ll}\text { 5. Conclusões e perspectivas } & 87\end{array}$

5. I. Conclusões . . . . . . . . . . . . . . . . 87

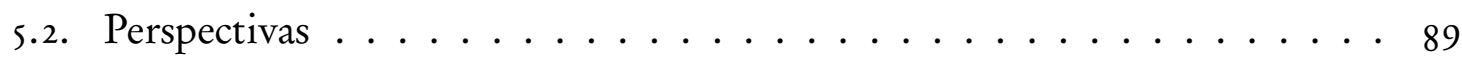

A. I. Adaptações mecânicas para a instalação da técnica de GISAXS . . . . . . . 91

B. I. Potencial químico da água na fase lamelar . . . . . . . . . . . 95

C. . . Modelo de bicamada rígida . . . . . . . . . . . . . . . . . 99

D. I. Artigo publicado no Journal of Applied Crystallography (J. Appl. Cryst.

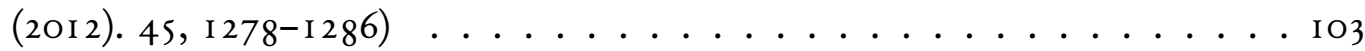

Barbara Bianca Gerbelli - Propriedades estruturais e elásticas de fases lamelares: O efeito da composição da membrana. 


\section{GLOSSÁRIO}

Moléculas anfifílicas: moléculas que apresentam duas regiões com diferentes solubilidades, uma hidrofílica e hidrofóbica.

Hidrofífica: Região que tem afinidade com moléculas polares, tal como à água.

Hidrofóbica: Região que não tem afinidade com moléculas polares, tal como à água.

Bicamada: Dupla camada formada por moléculas lipídicas em presença de um solvente.

Fases Lamelares e esmética: Arranjo de bicamadas periodicamente espaçadas com ordenamento posicional ao longo de uma direção, enquanto nas outras duas direções as moléculas anfifílicas podem apresentar diferentes arranjos.

$\boldsymbol{L}_{\alpha}$ : Fase lamelar que ocorre à temperatura mais alta, e alta hidratação. Nessa fase, as moléculas apresentam uma ordem líquida, com as cadeias carbônicas sem qualquer tipo de ordenamento.

$\boldsymbol{L}_{\boldsymbol{\beta}}$ e $\boldsymbol{P}_{\boldsymbol{\beta}}$ : Fases que as caudas carbôbicas apresentam um arranjo mais ordenado das cadeias carbônicas. Nessas duas fases as cadeias carbônicas estão estendidas e podem apresentar um certo grau de ordenamento posicional $2 \mathrm{D}$ de curto alcance, o que confere maior rigidez à bicamada.

Lipídios Zweteriônicos: Classe de lipídio que apresenta carga total neutra, porém, na região hidrofílica apresenta um momento de dipolo.

Barbara Bianca Gerbelli - Propriedades estruturais e elásticas de fases lamelares: O efeito da composição da membrana. 
$\delta_{M}:$ Espessura da bicamada

$\boldsymbol{\delta}_{\boldsymbol{w}}$ : Distância de separação entre as bicamadas, ou espessura da camada aquosa.

Periodicidade lamelar $(\boldsymbol{D})$ : Definida como: $\boldsymbol{\delta}_{M}+\boldsymbol{\delta}_{\boldsymbol{w}}$, representa o período de repetição de um padrão que é formado pela bicamada + camada aquosa.

$\phi_{l i p}$ : Fração volumétrica de lipídio.

SAXS: Espalhamento de raios X à baixo ângulo.

WAXS: Espalhamento de raios X à alto ângulo.

GISAXS: Espalhamento de raios X pôr incidência rasante.

Fator de estrutura (I(q)): Intensidade de luz espalhada.

Fator de estrutura $(\mathbf{S}(\mathbf{q}))$ : Descreve a repetição de um padrão que produz espalhamento de raios $\mathrm{X}$.

Fator de forma $(\mathbf{P}(\mathbf{q}))$ : Descreve características a forma do padrão que produz o espalhamento.

$\boldsymbol{\eta}$ : Parâmetro de Caillé associado às flutuações das membranas.

$\sigma_{D}$ : Amplitude de flutuação da distância de separação entre as bicamadas.

$\bar{B}$ : Módulo de compressibilidade $[\mathrm{Pa}]$

$\kappa:$ Módulo elástico de uma única membrana $\left[\boldsymbol{k}_{B} \boldsymbol{T}\right]$

$\boldsymbol{K}$ : Módulo de curvatura do "bulk" [J/m]

$\Delta \rho$ : Contraste de densidade eletrônica.

$\Delta \rho_{H}$ e ${ }_{T}$ : Contraste de densidade eletrônica entre cabeça polar e cauda apolar, respectivamente.

$\delta_{H}$ : Dimensão da região polar.

$\delta_{T}$ : Dimensão da região hidrofóbica.

$V$ : Potencial de interação entre as bicamadas.

$\boldsymbol{V}_{\boldsymbol{w}}$ : Potencial atrativo de van der Waals.

$\boldsymbol{V}_{\boldsymbol{H}}$ : Potencial repulsivo de curto alcance, nomeado de Potencial de Hidratação.

Barbara Bianca Gerbelli - Propriedades estruturais e elásticas de fases lamelares: O efeito da composição da membrana. 
$V_{E}$ : Potencial repulsivo de longo alcance, de natureza entrópica relacionado às flutuações das membranas.

$W$ : Constante de Hamaker, esta constante depende diretamente da composição da membrana.

$\boldsymbol{P}_{E}$ : Constante da amplitude do potencial estérico.

$\boldsymbol{P}_{\boldsymbol{H}}$ : Constante da amplitude do potencial de hidratação.

$\lambda_{H}$ : Constante de decaimento do potencial de hidratação.

$\lambda_{E}$ : Constante de decaimento do potencial estérico.

$\boldsymbol{W}_{\boldsymbol{c}}$ : Constante de Hamaker crítico.

$\boldsymbol{L}_{b}$ : Comprimento de Bjerrum do solvente

$I_{\text {amostra: }}$ : Espalhamento da fase lamelar dentro do capilar

$I_{\text {back }}$ : Espalhamento do capilar utilizado no experimento de espalhamento de raios X ("background")

$\boldsymbol{I}_{\text {ruido }}$ e $\boldsymbol{I}_{\text {sombra }}$ : Espalhamento que correspondem a corrente de fundo do equipamento e a sombra do feixe direto incidente no "beamstopper".

$\boldsymbol{I}_{\text {tratada: }}$ Espalhamento da fase lamelar depois da subtração dos "backgrounds".

$\boldsymbol{I}_{0}$ : Padrão secundário de espalhamento usado por ser um padrão cujo espalhamento não depende do ângulo de espalhamento na faixa angular de interesse.

$\boldsymbol{R H}$ : Umidade relativa dentro de um reservatório

$\boldsymbol{\mu}$ : Potencial químico

$\boldsymbol{P}$ : Pressão necessária para retirar uma molécula de água entre as bicamadas

$P_{0}$ : Pressão de vapor saturado.

$v_{w}$ : Volume parcial molar da água.

Barbara Bianca Gerbelli - Propriedades estruturais e elásticas de fases lamelares: O efeito da composição da membrana. 

CAPÍTULO 1

INTRODUÇÃO

\subsection{Motivação}

A arquitetura e função da membrana celular tem um papel fundamental para a regulação de suas funções e comunicação com o meio exterior. A formação de protrusões ou invaginações na membrana, poros e a formação de estruturas mais complexas como aparato de Golgi são controladas por forças elásticas e transições de fase estruturais [I], [2], [3], [4], [5]]. (Figura I.I)

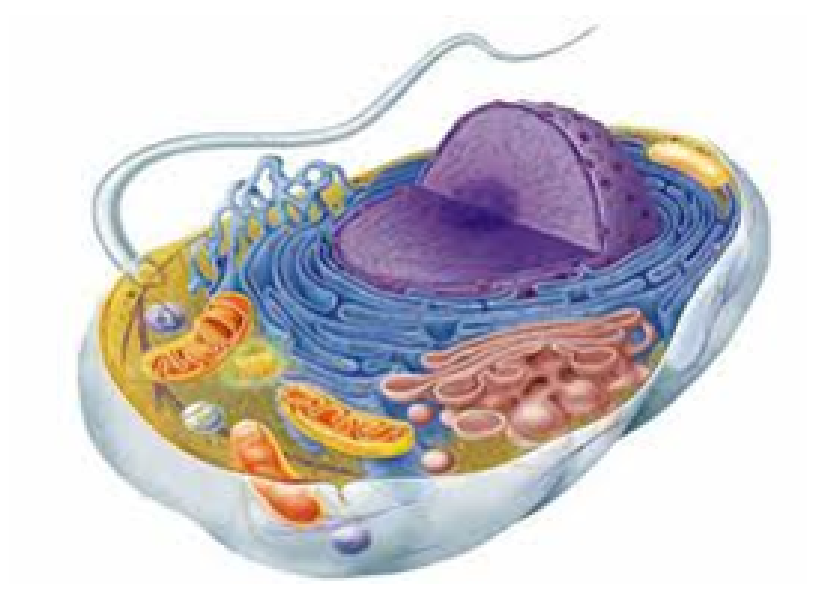

Figura i.ı.: Esquema de uma célula animal.

Mudanças na composição química da membrana são responsáveis por alterações em suas propriedades mecânicas [6], [7] que se manifestam em diferentes formas de organização tridimensional [8], [9] e influenciam processos de adesão de proteínas, fusão de mem-

Barbara Bianca Gerbelli - Propriedades estruturais e elásticas de fases lamelares: O efeito da composição da membrana. 
branas e interação entre proteínas membranares [6]. Flutuações locais de composição ou de concentração, chamados de "rafts" desempenham um papel importante na formação de protusões ou invaginações e estão associadas a mudanças locais na flexibilidade da membrana [6].
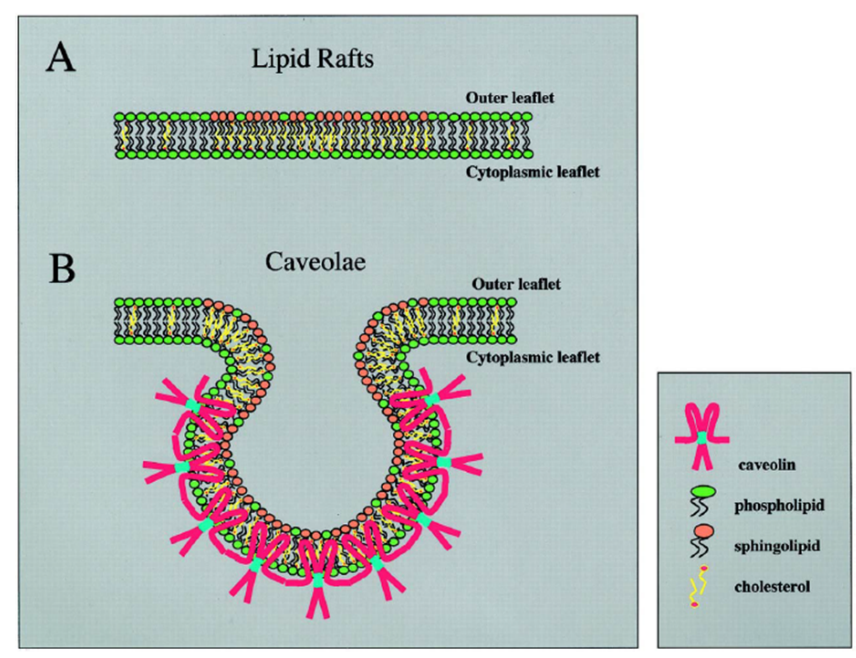

Figura I.2.: (A) Modelo esquemático de uma membrana de lipídios. (B) A formação da invaginação está ligada a formação de domínios ("rafts") ricos em caveolina (proteína membranaria) e colesterol extraído de [6]).

Sistemas lamelares são compostos por bicamadas de surfactantes regularmente espaçadas e tem sido bastante utilizados como sistema modelo para investigações de processos biológicos, pesquisa fundamental de interações entre membranas e para aplicações em biotecnologia. A estrutura periódica existe em apenas uma dimensão, enquanto nas outras duas dimensões, a bicamada comporta-se como um fluido. As flutuações térmicas que ocorrem na membrana dão origem á interações repulsivas resultantes do impedimento estérico mútuo quando as membranas estão próximas. Essas interações desempenham um papel fundamental na estabilização da fase lamelar uma vez que seu alcance e intensidade são comparáveis à interação atrativa de van der Waals [ Io], [ I I ], [ I 2], [ I3].

Experimentalmente foi observado que alguns sistemas lamelares apresentam um grande domínio de diluição, utilizando seja água ou óleo como solvente. Essa característica resulta da interação repulsiva de longo alcance devido a alta flexibilidade das bicamadas, que é obtida graças à adição de um co-surfactante á bicamada [I4] e [I s]. Do ponto de vista microscópico, a flexibilidade da membrana está relacionada à desordem orientacional e posicional das moléculas de surfactantes e também à dimensão da cadeia carbônica. Um exemplo do efeito da alteração da flexibilidade da membrana na estabilidade da fase lamelar é o sistema composto por DMPC e água, que atinge periodicidade lamelar máxima

Barbara Bianca Gerbelli - Propriedades estruturais e elásticas de fases lamelares: O efeito da composição da membrana. 
em torno de $45 \AA$, porém com a incorporação de um cosurfactante a estrutura lamelar estabiliza-se com periodicidade de até $200 \AA \AA$ [ I s].

As técnicas de espalhamento de raios $\mathrm{X}$ e nêutrons tem demonstrado serem ferramentas bastante úteis na descrição detalhada da estrutura lamelar [I6], [I7], [I8]. As flutuações térmicas destróem a ordem posicional de longo alcance e produzem um espalhamento difuso. A análise cuidadosa dos dados experimentais, incluindo o espalhamento difuso, pode fornecer informações relevantes, não apenas dos parâmetros estruturais, mas também das propriedades elásticas da estrutura lamelar [18] [19].

\subsection{Estado da arte e objetivos}

Existe na literatura, desde a década de $\mathbf{8 0}$, o interesse de compreender o mecanismo estrutural e funcional de macromoléculas que estão presente em sistemas biológicos, tais como, descrito anteriormente.

$\mathrm{Na}$ literatura existem vários trabalhos voltados para o estudo de propriedades estruturais de membranas utilizando vesículas ou fases lamelares [20] compostas por um único fosfolipídio, como DPPC (dipalmitoil fosfatidilcolina), ou DMPC (dimiristoil fosfatidilcolina), entre outros. Uma abordagem bastante utilizada para o estudo das interações entre as bicamadas é a preparação da fase lamelar em uma solução de polímeros [2 I ], onde a pressão osmótica exercida sobre as membranas é controlada pela concentração de polímeros em solução. Essa abordagem combinada com espalhamento de raios $\mathrm{X}$ permite a determinação da separação entre as membranas em equilíbrio para uma dada pressão osmótica e os resultados podem ser utilizados para obtenção de parâmetros que caracterizam as interações entre as membranas, tais como constante de Hamaker, constante de rigidez, e módulo de compressão [22], [23]. Existem ainda alguns trabalhos onde a composição da membrana é variada introduzindo combinação de fosfolipídios [2] ou ainda colesterol [24].

Partindo desse cenário este trabalho tem como objetivo apresentar um estudo sistemático das propriedades estruturais e elásticas de um sistema lamelar composto de lecitina e um co-surfactante comercial, Simulsol ${ }^{\circledR}$, que é uma mistura de moléculas de cadeia única etoxiladas. Devido ao caráter zwiterionico dos grupos polares da lecitina e o caráter não-iônico do Simulsol, a carga total da membrana permanece neutra. A incorporação sistemática de moléculas com cadeia carbônica simples deve promover aumento na flexibilidade da membrana, porém há um efeito secundário relacionado as cadeias etoxiladas que as compõem. Essas cadeias são hidrofílicas e estendem-se na camada aquosa, no espaço

Barbara Bianca Gerbelli - Propriedades estruturais e elásticas de fases lamelares: O efeito da composição da membrana. 
entre as bicamadas, introduzindo uma repulsão extra devido aos efeitos estéricos.

Os parâmetros relevantes que caracterizam a estrutura lamelar são determinados para membranas com composição variando de ı oo\% de lecitina á ı $\mathrm{da}$ linha de diluição. $\mathrm{Na}$ análise dos dados experimentais de espalhamento de raios $\mathrm{X}$, o perfil de densidade eletrônica é modelado utilizando um conjunto de funções Gaussianas simétricas, cujas amplitude e largura são ajustadas. O método de ajuste é aplicado diretamente á intensidade espalhada, utilizando uma rotina de mínimos quadrados com vínculo, o que permite a determinação simultânea dos fatores de forma e estrutura, além de vários parâmetros que descrevem o modelo, tais como a espessura da bicamada, a distância de separação entre as bicamadas e o parâmetro de Caillé que relaciona as duas constantes elásticas.

A abordagem experimental proposta neste trabalho nos permite investigar o efeito das interações estéricas na estabilidade da fase lamelar pelas mudanças introduzidas na composição da membrana. Por um lado, a membrana torna-se mais fluida pela incorporação de moléculas de cadeia simples, e por outro lado, há um aumento da interação repulsiva pela presença de uma cabeleira na interface da membrana. A varredura da linha de diluição nos permite investigar diferentes regimes de interações, desde um regime confinado, com as membranas muito próximas, até regimes muito diluídos, onde as interações repulsivas devido às flutuações térmicas devem ter um papel predominante.

Um outro aspecto importante deste trabalho consiste no método utilizado para a análise dos dados experimentais de espalhamento de raios X que levam á determinação do perfil de densidade eletrônica, independente de considerações prévias sobre a configuração das cadeias carbônicas e grupos polares. Assim, mudanças importantes que ocorrem na interface entre a bicamada e a camada aquosa, dependentes da composição da membrana, podem ser detectadas. Esse método de análise foi aplicado inicialmente à fase lamelar com membranas compostas apenas de lecitina e os resultados obtidos revelam que o processo de desidratação da fase lamelar promove alterações na estrutura da bicamada. Além da técnica usual de SAXS aplicada ao estudo das propriedades estruturais da fase lamelar, neste trabalho foi desenvolvida uma montagem experimental para experimentos de difração de raios $\mathrm{X}$ em filmes orientados, na geometria de reflexão em incidência rasante (GISAXS). Os filmes orientados são colocados em uma câmara com umidade controlada, o que permite fazer um estudo termodinâmico da hidratação (ou desidratação) do filme para uma dada composição da membrana. A partir da umidade relativa, pode-se determinar a pressão osmótica ou o potencial químico da água na fase lamelar e esses resultados dão acesso à determinação do módulo de compressibilidade $(\overline{\boldsymbol{B}})$.

Barbara Bianca Gerbelli - Propriedades estruturais e elásticas de fases lamelares: O efeito da composição da membrana. 
O comportamento do parâmetro de Caillé ao longo da linha de diluição é obtido para as diferentes composições da membrana e revela a existência de dois regimes de interação distintos entre as bicamadas. Combinando os valores obtidos para o parâmetro de Caillé e os valores obtidos para o módulo de compressão é possível, então, obter informações sobre a constante de rigidez da membrana e o seu comportamento em função da hidratação.

\subsection{Organização}

No Capítulo 2 serão apresentados modelos teóricos que descrevem as interações entre as membranas e suas propriedades elásticas. Nesse capítulo será apresentado também o método utilizado para análise dos resultados experimentais de espalhamento de raios $\mathrm{X}$. Os protocolos utilizados para a preparação das amostras para os experimentos de SAXS e GISAXS são descritos no Capítulo 3, assim como detalhes sobre a montagem de GISAXS desenvolvida neste trabalho.

No Capítulo 4 são apresentados os resultados obtidos neste trabalho, organizados em duas partes. Em primeiro lugar serão apresentados os resultados obtidos para as membranas compostas apenas de lecitina, com sua caracterização estrutural. Os resultados obtidos são comparados a resultados existentes na literatura. Em seguida são apresentados os resultados obtidos para as membranas onde foi introduzido co-surfactante em diferentes quantidades. O efeito da adição do co-surfactante é analisado observando-se o comportamento dos parâmetros estruturais, tais como espessura da bicamada, período lamelar, fator de forma, e do parâmetro de Caillé.

Em segundo lugar são apresentados os resultados dos estudos termodinâmicos, onde a fase lamelar está em contato com um reservatório de vapor de água e o processo cinético de hidratação ou desidratação da fase lamelar pode ser estudado. A partir desses experimentos é determinado o módulo de compressibilidade, que combinado com o parâmetro de Caillé leva a determinação da constante de rigidez, para duas composições de membrana.

O Capítulo s traz uma discussão dos principais resultados obtidos neste trabalho e a contribuição desses resultados para a compreensão das propriedades elásticas das membranas de lipídios.

Barbara Bianca Gerbelli - Propriedades estruturais e elásticas de fases lamelares: O efeito da composição da membrana. 

CAPÍTULO 2

FUNDAMENTAÇÃO TEÓRICA

\subsection{Fenômenos de auto-organização}

Algumas moléculas, chamadas de anfifílicas, apresentam regiões com diferentes solubilidades, conforme mostrado de maneira esquemática na figura 2. I, onde pode-se identificar uma parte polar, hidrofílica e uma parte apolar, hidrofóbica. Na presença de um solvente, e a partir de uma certa concentração, essas moléculas tendem a se auto-organizar formando estruturas mais complexas. O tipo de estrutura que será formado depende de

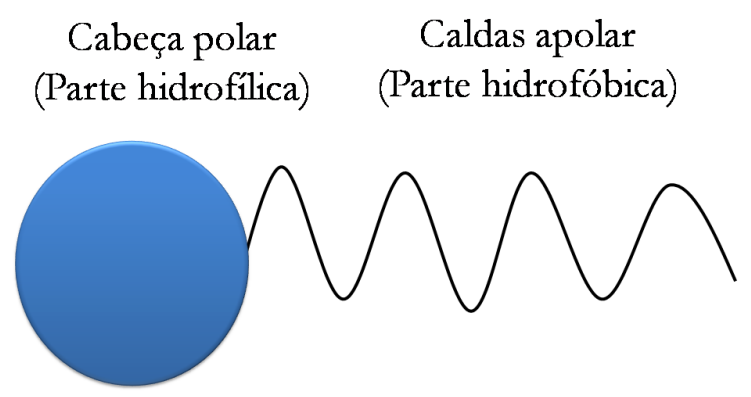

Figura 2. I.: Desenho esquemático de uma molécula anfifílica.

fatores geométricos da molécula, (figura 2.2) ,ou seja, da razão entre o volume ocupado pela parte polar e o volume ocupado pela parte apolar. Assim, utilizando a água como solvente, moléculas com geometria tipo cone tendem a formar micelas esféricas, com a

Barbara Bianca Gerbelli - Propriedades estruturais e elásticas de fases lamelares: O efeito da composição da membrana. 
parte polar na superfície exposta ao solvente, enquanto moléculas com geometria mais próxima de um cilindro tendem a formar bicamadas, com as cadeias carbônicas voltadas para o interior da bicamada. [25]

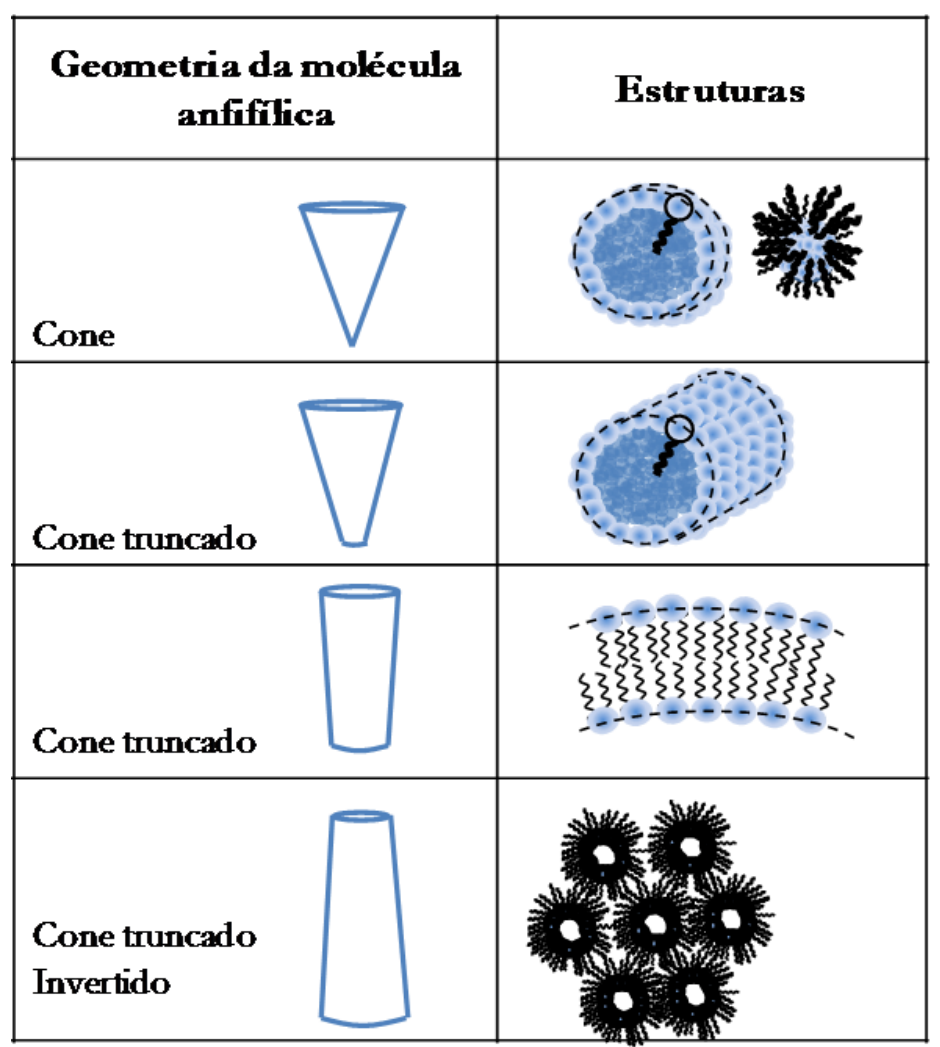

Figura 2.2.: Estruturas formadas a partir do fenômeno de auto-organização de moléculas anfifílicas.

\subsection{Fosfolopídios}

A palavra lipídio vem do grego “lipos” que significa gordura. Os lipídios são moléculas anfifílicas que possuem duas cadeias carbônicas ligadas a um grupo polar e na presença de água, devido à alta hidrofobicidade das cadeias carbônicas, tendem a formar bicamadas (Figura 2.3).

A membrana celular é um exemplo de uma bicamada, composta de diferentes tipos de lipídios, majoritariamente fosfolipídios, isto é que apresentam um grupo fosfato na parte polar. Na membrana celular os fosfolipídios mais abundantes são fosfatidilcolina, fosfatidilserina e fosfatidiletaloamina, com cadeias carbônicas variando entre 16 á i 8 car-

Barbara Bianca Gerbelli - Propriedades estruturais e elásticas de fases lamelares: O efeito da composição da membrana. 


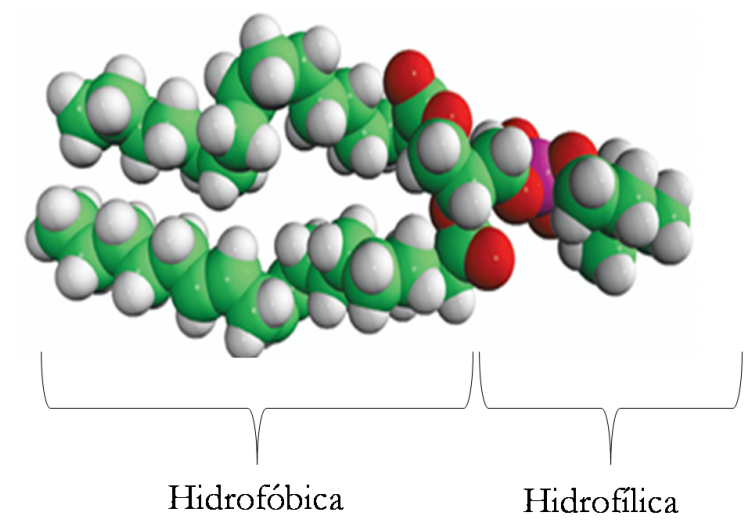

Figura 2.3.: Estrutura da molécula da fosfatidilcolina.

bonos [26]. Quanto ao grupo polar, estes podem ou não apresentar carga, ou ainda podem apresentar um momento de dipolo elétrico, e nesse caso são denominados de zwiterioni$\cos [27]$.

A membrana celular desempenha um papel muito importante no aparecimento da vida, pois ao mesmo tempo em que delimita uma região onde podem ser acomodadas outras macromoléculas que passam a interagir, ela é também bastante flexível, pois não existem ligações covalentes entre as moléculas de fosfolipídios. A membrana serve também como substrato para outras biomoléculas, como proteínas, enzimas e receptores que permitem a comunicação do meio externo com o meio interno.

\subsection{Fases Lamelares liotrópicas}

A fase lamelar é formada por um arranjo de bicamadas periodicamente espaçadas, ou seja, há um ordenamento posicional ao longo de uma direção, enquanto nas outras duas direções as moléculas anfifílicas podem apresentar diferentes arranjos. Esse é um sistema liotrópico onde as transições de fase ocorrem principalmente por variações de concentração relativa, mas podem também ocorrer por variação de temperatura. Essa estrutura é conhecida como fase esmética observada em cristais líquidos [28].

Os primeiros estudos sobre a morfologia das fases lamelares foram conduzidos por Luzatti [29], nos anos 6o e 70, com a identificação de três tipos de fases lamelares, $\boldsymbol{L}_{\boldsymbol{\alpha}}$, $\boldsymbol{L}_{\boldsymbol{\beta}}$ e $\boldsymbol{P}_{\boldsymbol{\beta}}$, que se distinguem pelos diferentes tipos de arranjo adotados pelas moléculas na bicamada (Figura 2.4). A fase $\boldsymbol{L}_{\alpha}$, é a que ocorre à temperatura mais alta, e alta hidratação. Nessa fase, as moléculas apresentam uma ordem líquida, com as cadeias carbônicas sem qualquer tipo de ordenamento e por isso essa fase é também chamada de fase lamelar

Barbara Bianca Gerbelli - Propriedades estruturais e elásticas de fases lamelares: O efeito da composição da membrana. 
fluida. As outras duas fases se caracterizam por um arranjo mais ordenado das cadeias carbônicas, como mostrado na figura 2.4. Nessas duas fases as cadeias carbônicas estão estendidas e podem apresentar um certo grau de ordenamento posicional $2 \mathrm{D}$ de curto alcance, o que confere maior rigidez à bicamada.
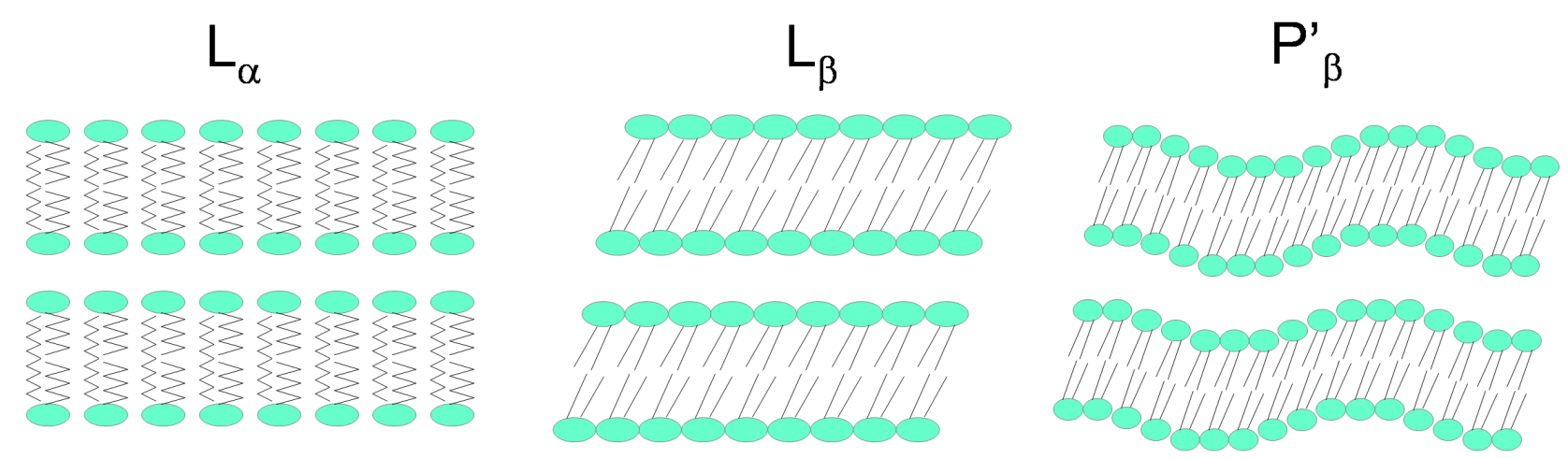

Figura 2.4.: Diferentes tipos de organização das moléculas de lipídios nas fases lamelares

As transições de fase podem ocorrer por variação da temperatura ou quantidade de água, como ilustrado na figura 2.5 para o fosfolipídio DMPC [30], [ I 4]. De modo geral, o aumento do número de carbonos na cadeia carbônica resulta em temperaturas de transição mais elevadas. [3I][32]

\subsection{Interações entre bicamadas em fases lamelares}

A estabilização da estrutura lamelar pode ser descrita no contexto clássico da teoria DLVO, como resultado do balanço entre as interações eletrostáticas devido à dupla camada e interações atrativas de van der Waals [33]. Experimentalmente, foi observada uma força repulsiva extra para pequenas distâncias de separação entre as bicamadas, com decaimento exponencial, e alcance da ordem de 2 Å. A natureza dessa força, denominada de força de hidratação, no entanto, ainda é um tema controverso na literatura, [25] [34] [35] e existem várias proposições que consideram a estruturação dos grupos polares na interface ou polarização e organização das moléculas de água [36] [37] [34]. Assim a energia de interação entre as bicamadas poderia ser escrita diretamente em termos de um potencial efetivo (por unidade de superfície), como a soma dos potencias de van der Waals $\left(\boldsymbol{V}_{\boldsymbol{W}}\right)$, eletrostático $\left(\boldsymbol{V}_{\boldsymbol{E}}\right)$ e um potencial de hidratação $\left(\boldsymbol{V}_{\boldsymbol{H}}\right)$. [22]

Barbara Bianca Gerbelli - Propriedades estruturais e elásticas de fases lamelares: O efeito da composição da membrana. 


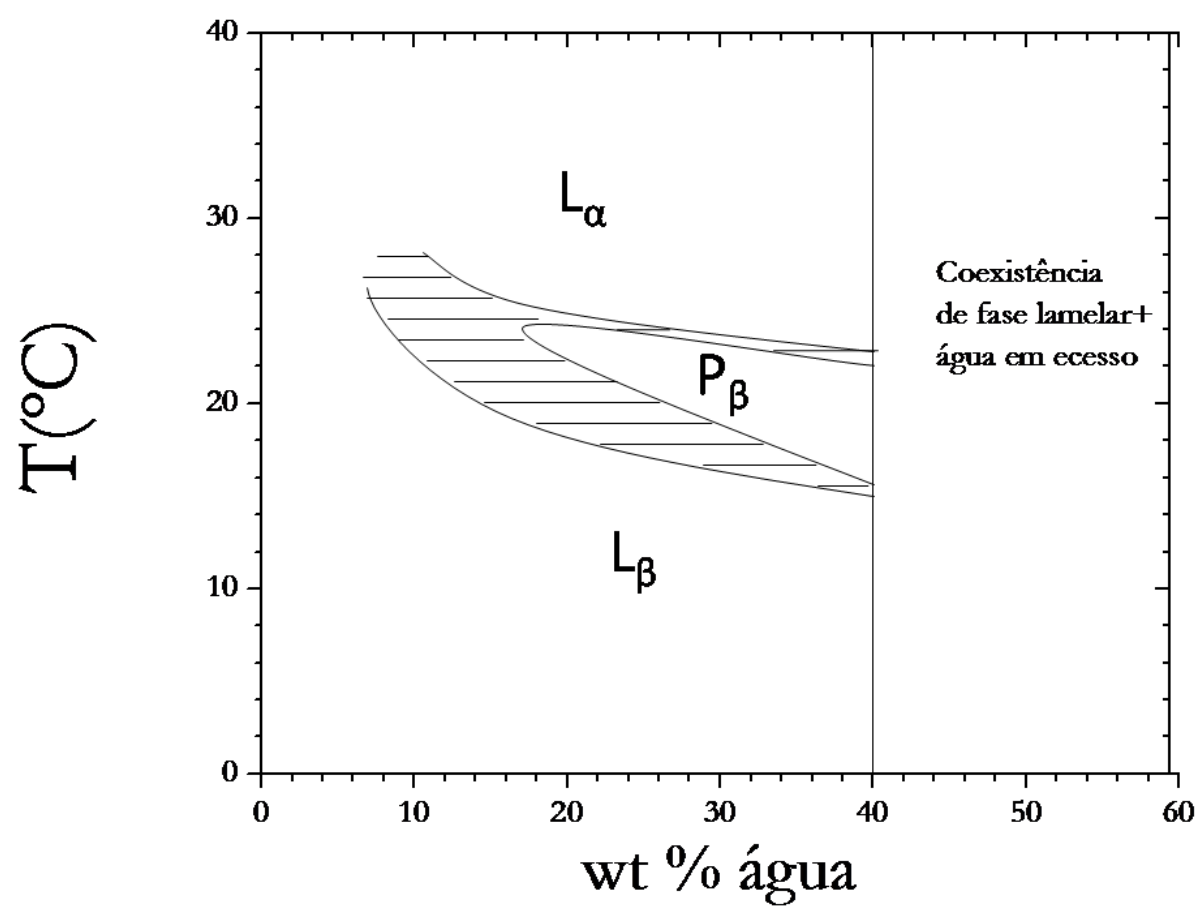

Figura 2.5.: Diagrama de fases utilizando raios X em amostras de DMPC (adaptada de [I4]).

$$
V=-\frac{W}{12 \pi}\left[\frac{1}{D^{2}}-\frac{2}{\left(D+\delta_{w}\right)^{2}}+\frac{1}{\left(D+2 \delta_{w}\right)^{2}}\right]+P_{H} e^{-\frac{\delta_{w}}{\lambda_{H}}}+P_{E} e^{-\frac{\delta_{w}}{\lambda_{E}}}
$$

onde $\delta_{w}$ é a distância de separação entre as bicamadas de espessura $\boldsymbol{\delta}_{M}$. O alcance das interações de eletrostática e hidratação é dado pelas constantes $\boldsymbol{\lambda}_{\boldsymbol{E}}$ e $\boldsymbol{\lambda}_{\boldsymbol{H}}$, e, W, $\boldsymbol{P}_{\boldsymbol{E}}$ e $\boldsymbol{P}_{\boldsymbol{H}}$ representam a intensidade das interações de van de Waals, eletrostática e de hidratação, respectivamente.

Como a constante de Hamaker (W) é positiva, de acordo com essa descrição, o potencial efetivo apresenta sempre um mínimo, que corresponde a um conjunto de duas membranas interagindo e separadas por uma dada distância, como resultado da interação atrativa de van der Waals. Quando há um equilíbrio entre as interações repulsivas e atrativas, a distância de separação atinge um valor máximo, que é chamado de limite de hidratação. Para alguns sistemas lamelares, no entanto, observa-se experimentalmente que a distância entre as bicamadas aumenta indefinidamente com a hidratação, ou seja não existe um mínimo no potencial de interação. [38] [39] [ I I]

Para explicar essas observações, Helfrich [40] propôs a existência de uma interação re-

Barbara Bianca Gerbelli - Propriedades estruturais e elásticas de fases lamelares: O efeito da composição da membrana. 
pulsiva de longo alcance, de natureza entrópica. A amplitude das flutuações de ondulação é reduzida quando as membranas são aproximadas e isso resulta na redução da entropia, porém esta é acompanhada do aumento da interação repulsiva. A energia elástica por unidade de volume devido às flutuações pode ser escrita usando a teoria elástica de cristais líquidos esméticos e para pequenas amplitudes de flutuação em torno de uma posição de equilíbrio (Fig. 2.8), pode ser escrita como:

$$
F=B \frac{1}{2}\left(\frac{\partial u}{\partial z}\right)^{2}+K \frac{1}{2}\left(\frac{\partial^{2} u}{\partial y^{2}}+\frac{\partial^{2} u}{\partial x^{2}}\right)^{2}
$$

onde $\mathrm{u}(\mathrm{x}, \mathrm{y})$ é amplitude da flutuação da separação entre as bicamadas em torno da posição de equilíbrio, B é definido como o módulo de compressão da camada (bicamada + água) à concentração constante, e K é o módulo de curvatura ou "splay" da estrutura lamelar. A constante $\boldsymbol{B}$ leva em conta as interações que fazem com que camadas vizinhas permaneçam ligadas, portanto, para uma camada isolada o valor de $\boldsymbol{B}$ é igual a zero [4I].

Considerando que as interações entre as membranas devem-se apenas aos efeitos de volume excluído, a energia de interação entre as bicamadas devido a esses efeitos, segundo o modelo de Helfrich, é dada por [40]:

$$
V_{s}=\mu \frac{\left(k_{B} T\right)^{2}}{\kappa \delta_{w}^{2}}
$$

onde $\boldsymbol{\kappa}$, é o módulo elástico de uma única membrana, ( também chamado de módulo de rigidez da membrana) [40] e está relacionado com a constante elástica $\mathrm{K}$, que representa o módulo de curvatura do "bulk" pela expressão:

$$
\kappa=K D
$$

Somando-se todas as contribuições, o potencial de interação entre as bicamadas poderia ser escrito então, como:

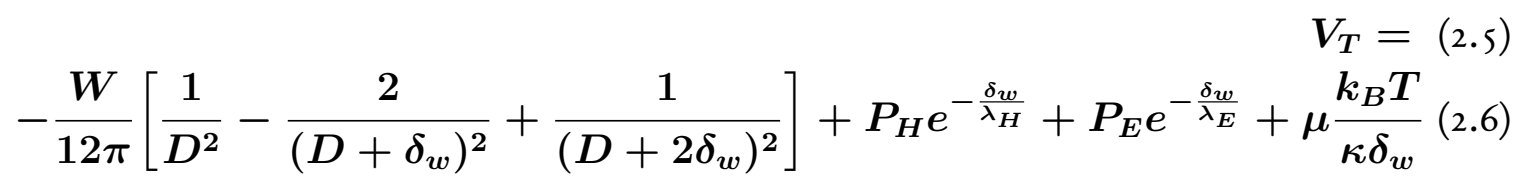

A simples adição da expressão obtida por Helfrich à expressão do potencial, no entanto,

Barbara Bianca Gerbelli - Propriedades estruturais e elásticas de fases lamelares: O efeito da composição da membrana. 
ainda não permite a descrição satisfatória das propriedades de transição de fase do sistema lamelar, pois esse potencial apresenta um mínimo. Portanto, poderia se esperar uma transição de primeira ordem entre um sistema de membranas ligadas e interagentes, para um sistema de membranas completamente separadas. Alguns trabalhos utilizam outras abordagens, como métodos estatísticos ou variacionais para avaliar o balanço entre as diferentes interações, em função da constante de Hamaker (W) ou da constante de rigidez da membrana [Io] [ I 2] [39] [42]. Essas abordagens resultam na previsão de uma transição de segunda ordem, de um sistema ligado para um sistema não ligado, com a distância de separação entre as membranas divergindo quando a constante de Hamaker assume um valor crítico $\boldsymbol{W}_{c}, \boldsymbol{a} \approx\left(\boldsymbol{W}-\boldsymbol{W}_{c}\right)$ [1 2] [39].. Essa transição também poderia ocorrer pela diminuição da constante de rigidez da membrana como mostrado na figura 2.6 [ I I ]

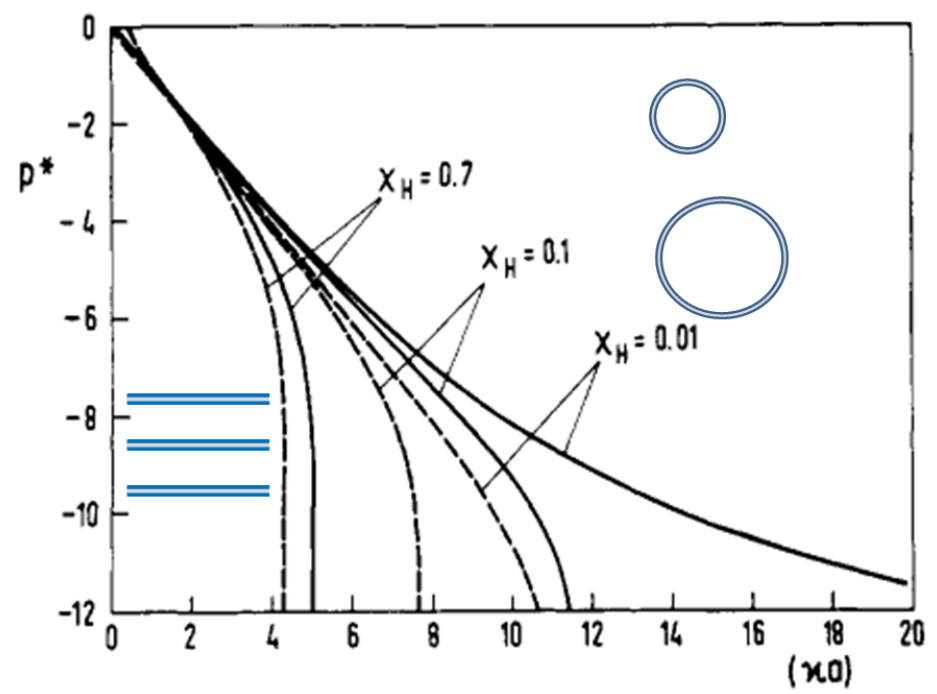

Figura 2.6.: Previsão teórica para o comportamento da pressão (adimensional) em função da distância de separação entre as bicamadas ( $\chi_{0}$, adimensional) para diferentes parâmetros $\chi_{\boldsymbol{H}}$, onde $\chi_{\boldsymbol{H}}$ é um parâmetro adimensional proporcional à $\kappa^{3}$ Figura adaptada de [42].

Barbara Bianca Gerbelli - Propriedades estruturais e elásticas de fases lamelares: O efeito da composição da membrana. 


\subsection{Teoria de espalhamento de raios $X$ em fases lamelares}

Desde o trabalho pioneiro de Luzatti [29] a técnica de espalhamento de nêutrons e raios $\mathrm{X}$ tem sido empregada para o estudo de vesículas e sistemas lamelares [17] [22] [43]. A posição dos picos de Bragg permite a determinação da periodicidade lamelar, porém devido às flutuações térmicas, há também um espalhamento difuso e esses picos tornam-se largos e não podem ser descritos por funções delta. A análise da intensidade de espalhamento, incluindo o espalhamento difuso pode fornecer uma descrição mais detalhada da fase lamelar quanto à sua estrutura global, assim como das flutuações térmicas.

Métodos de análise dos dados experimentais de espalhamento que levem em conta não apenas os picos de Bragg, mas também o espalhamento difuso têm recebido bastante atenção [44], pois informações mais detalhadas podem ser obtidas, mesmo quando existem poucas ordens de difração. Esses métodos levam em conta o máximo possível do sinal detectado experimentalmente para garantir a confiabilidade nos valores obtidos, e ao mesmo tempo devem ser aplicáveis à uma variedade de situações, como por exemplo, membranas com diversas composições, formando vesículas ou estruturas lamelares.

A intensidade de espalhamento de um conjunto de camadas espaçadas periodicamente está relacionada com a modulação na densidade eletrônica e pode ser descrita como o resultado da convolução da função que descreve a forma e contraste do objeto; fator de forma $(\mathrm{P}[\mathrm{q}])$ e da função fator de estrutura $(\mathrm{S}[\mathrm{q}])$ que descreve o arranjo periódico dos objetos e também carrega informações sobre a interação entre eles;

$$
I_{o}(q)=\frac{S(q)|P(q)|^{2}}{q_{z}{ }^{2}}
$$

$\mathrm{Na}$ equação acima, $q$ é o vetor de onda de espalhamento relacionado ao ângulo de espalhamento $2 \boldsymbol{\theta}$ pela expressão, $q=(4 \pi / \lambda) \operatorname{sen} \theta$, onde $\boldsymbol{\lambda}$ é o comprimento de onda da radiação incidente e o fator $\boldsymbol{q}^{2}$ no denominador é uma correção para espalhadores isotrópicos. A função $\mathrm{S}[\mathrm{q}]$ corresponde à transformada de Fourier da função de rede descrevendo o arranjo periódico unidimensional, chamado de fator de estrutura. Uma aproximação usual consiste em desacoplar o fator de forma e o fator de estrutura e trata-los separadamente. A seguir serão apresentados modelos para descrever o fator de estrutura e o fator de forma, assim como as aplicações para dados experimentais.

Barbara Bianca Gerbelli - Propriedades estruturais e elásticas de fases lamelares: O efeito da composição da membrana. 


\subsubsection{Fator de estrutura e parâmetro de Caillé}

De acordo com a Teoria de Caillé modificada, [45] o fator de estrutura para um sistema multilamellar, com periodicidade D é descrito de maneira satisfatória para uma média estatística:

$S_{0}(q)=1+2 \sum_{k=1}^{N-1}\left(1-\frac{n}{N}-k\right) \cos (k q D) \exp \left[-\left(\frac{D}{2 \pi}\right)^{2} q^{2} \eta \gamma\right](n \pi)^{-\left(\frac{D}{2 \pi}\right) q^{2} \eta} \cdot(2.8)$

$\mathrm{Na}$ equação acima, $\mathrm{N}$ representa o número de camadas correlacionadas na estrutura multicamadas, $\gamma$ é a constante de Euler e $\boldsymbol{\eta}$ é o parâmetro de Caillé. Esse parâmetro relaciona as constantes elásticas $\bar{B}$ e $\mathrm{K}$ :

$$
\eta=\frac{q_{0}^{2} k_{B} T}{8 \pi \sqrt{K \bar{B}}}
$$

onde $\mathrm{K}$ é o módulo de curvatura e $\overline{\boldsymbol{B}}$ o módulo de compressibilidade à potencial químico constante.

A figura 2.7 representa de maneira esquemática o efeito das flutuações térmicas na função S[q]. Para uma estrutura cristalina unidimensional, formada por bicamadas rígidas, periodicamente espaçadas, a função $\mathrm{S}[\mathrm{q}]$ seria descrita por picos de Bragg de mesma largura, centrados em $\boldsymbol{q}_{n}=2 \pi / D$, onde n corresponde à ordem de difração. A largura do pico é inversamente proporcional à dimensão do domínio; $L=N D$, onde $N$ é o número de camadas correlacionadas. Para $N \rightarrow \infty$, a largura dos picos tende a zero, e a função $S[q]$ se aproxima de funções delta, com largura finita, determinada pela resolução instrumental.

Pequenas flutuações na posição das bicamadas em torno da posição de equilíbrio, assim como flutuações do tipo "bending” resultam em alargamento dos picos e decaimento da intensidade dos picos. Na figura 2.7 (b), o fator ${\sigma_{D}}^{2}$ representa a amplitude quadrática média das flutuações do espaçamento entre as bicamadas em torno da posição de equilíbrio (Figura 2.8):

$$
\left.\sigma_{D}^{2}=<\left(u_{n}\right)-u_{0}\right)^{2}>
$$

Barbara Bianca Gerbelli - Propriedades estruturais e elásticas de fases lamelares: O efeito da composição da membrana. 

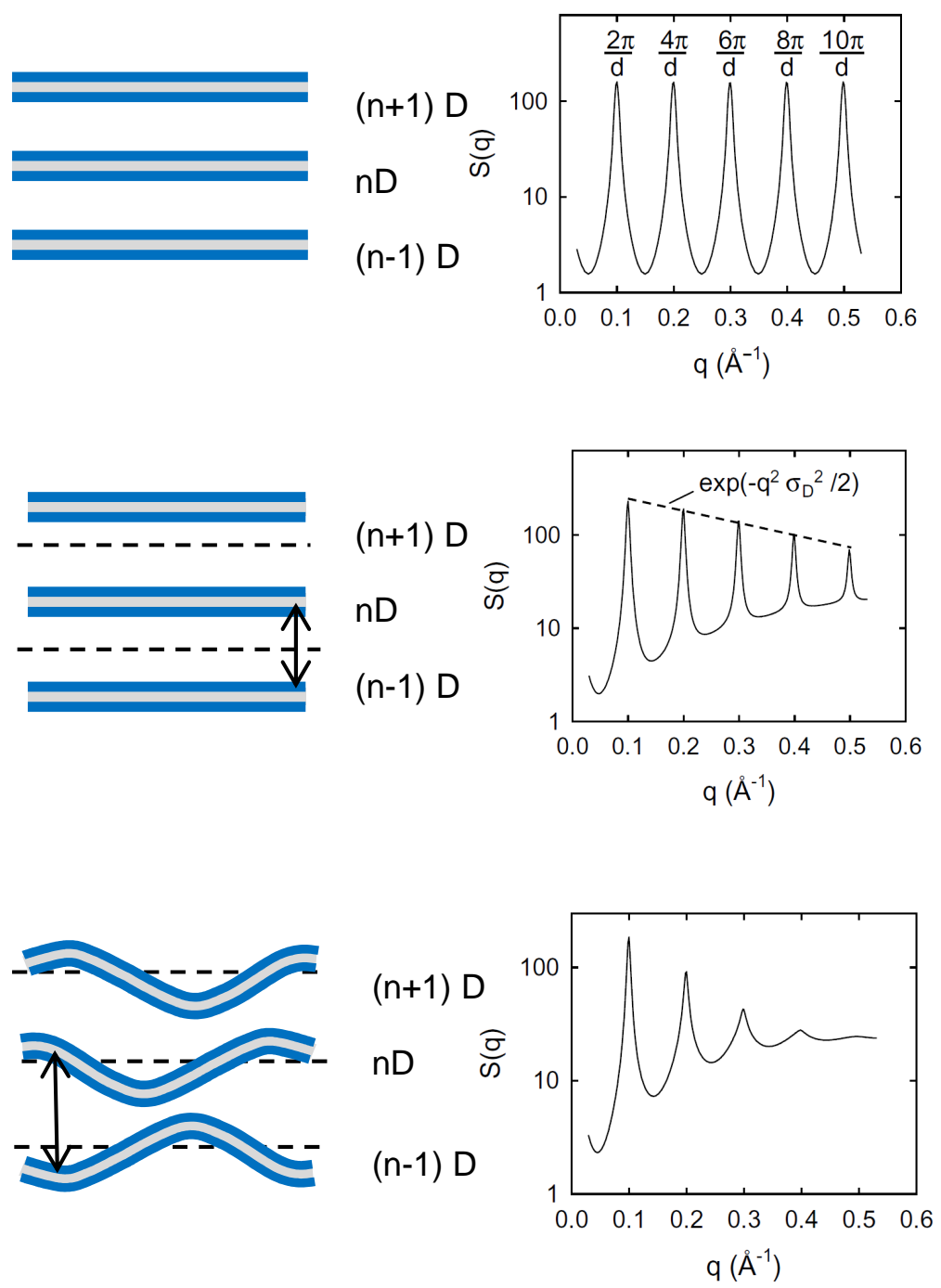

Figura 2.7.: Representação esquemática da fase lamelar e a intensidade de espalhamento I (q) correspondente :(a) para membranas rígidas; (b) com flutuações da periodicidade lamelar (c) com flutuações de ondulações da membrana. Figura adaptada de [19]

que por sua vez, está relacionado ao parâmetro de Caillé pela relação: [22]

$$
\sigma_{D}^{2}=\frac{\eta D^{2}}{\pi^{2}}
$$

Barbara Bianca Gerbelli - Propriedades estruturais e elásticas de fases lamelares: O efeito da composição da membrana. 

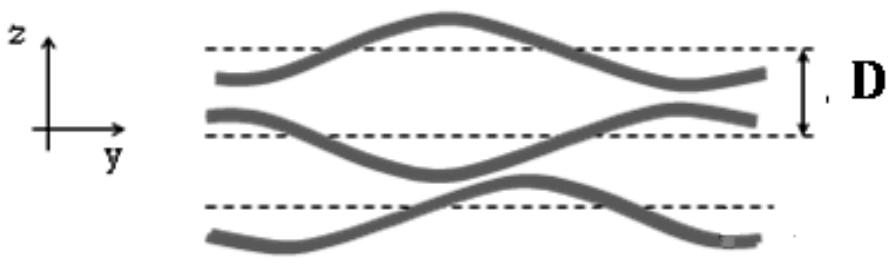

Figura 2.8.: Flutuações térmicas das membranas em uma fase lamelar de periodicidade igual a D.

\subsubsection{Fator de forma}

A função $\mathrm{P}[\mathrm{q}]$ é a transformada de Fourier da modulação de densidade eletrônica ao longo de uma bicamada. Alguns métodos de análise dos dados experimentais consideram que na vizinhança dos picos de Bragg o valor da função $\mathrm{P}[\mathrm{q}]$ é aproximadamente constante; $\mathrm{P}[\mathrm{q}] \approx \boldsymbol{P}_{\boldsymbol{n}}$. Com essa aproximação a densidade eletrônica pode ser escrita como:

$$
\rho(z)=\sum_{n=1}^{n_{\max }} \alpha_{n}\left|P_{n}\right| \cos \left[\frac{2 \pi n z}{D}\right]
$$

onde $\boldsymbol{\alpha}_{n}= \pm 1$ é o fator de fase, $\boldsymbol{P}_{\boldsymbol{n}}$ corresponde à raiz quadrada da amplitude do pico. Para minimizar os erros de truncamento na série de Fourier, a aplicação da equação acima requer a existência de pelo menos 4 picos de Bragg, o que não é muito comum em sistemas lamelares, principalmente no regime muito hidratado. A grande limitação dessa aproximação, no entanto, está no fato de que $\mathrm{P}[\mathrm{q}]$ varia bastante, sobretudo na região de picos de Bragg de ordem mais baixa, evidenciado pela assimetria nos picos. Esse modelo não é capaz de ajustar a cauda difusa desses picos [19].

Uma abordagem alternativa e simples foi proposta por Nallet et all [ [ 8], e consiste em descrever a modulação na densidade eletrônica por meio de funções degrau;

$$
P(q)=\frac{4 \Delta \rho_{H}^{2}}{q^{2}}\left\{\sin \left(q\left(\delta_{H}+\delta_{T}\right)\right)-\sin \left(q \delta_{T}\right)+\frac{\Delta \rho_{T}}{\Delta \rho_{H}} \sin \left(q \delta_{T}\right)\right\}^{2} .
$$

Onde $\Delta \rho_{H}$ e $\Delta \rho_{T}$, representam a densidade eletrônica do grupo polar e da cauda carbônica, respectivamente e $\delta_{H}$ e $\delta_{T}$ a dimensão (ao longo do eixo z), do grupo polar e da cauda carbônica, respectivamente de acordo com figura 2.9.

Esse modelo, embora aproximativo, é fácil de ser implementado para fins de ajustes aos dados experimentais, e fornece uma descrição quantitativa satisfatória na região de

Barbara Bianca Gerbelli - Propriedades estruturais e elásticas de fases lamelares: O efeito da composição da membrana. 


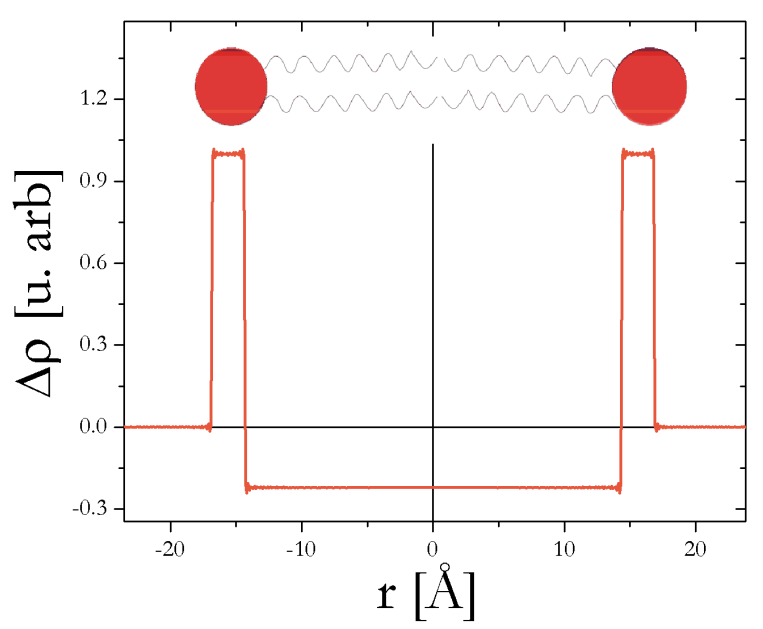

Figura 2.9.: Representação esquemática do contraste de densidade eletrônico da bicamada obtida pelo modelo de 3 degraus. Nesse modelo a parte positiva do contraste se refere a cabeça polar e negativa as caudas carbônicas.

pequenos ângulos.

Uma descrição mais detalhada da modulação da densidade eletrônica pode ser obtida utilizando funções gaussianas, onde a função $\rho(z)$ apresenta variações mais suaves [17]. Usando duas funções gaussianas, a parte central da bicamada é representada por uma gaussiana com amplitude negativa em relação à agua, centrada no meio da bicamada, e o grupo polar é representado por uma segunda gaussiana, com amplitude positiva, cujo pico corresponde à posição da cabeça polar. A suavização da função $\rho(z)$ melhora bastante a qualidade dos ajustes aos resultados experimentais, mas ainda falha na região de valores altos de $q$, e com dados de alta resolução. Uma nova abordagem consiste na utilização de um conjunto de funções gaussianas, regularmente espaçadas, aliado a um método de otimização que determina a amplitude e largura das funções que melhor ajustam os dados experimentais. Esse método, chamado de método de deconvolução gaussiana [44], mostrou-se bastante adequado para descrever bicamadas simétricas, com o uso de 4 funções gaussianas para descrever metade da bicamada. De acordo com esse modelo a densidade eletrônica passa a ser descrita por:

Barbara Bianca Gerbelli - Propriedades estruturais e elásticas de fases lamelares: O efeito da composição da membrana. 


$$
\begin{array}{r}
\rho(z)=\sum_{n=1}^{4} \frac{a_{n}\left[G_{s}\left(z, z_{n}, \sigma_{n}\right)+G_{s}\left(z-z_{n}, \sigma_{n}\right)\right]}{1+\delta_{i j}} \\
\text { podendo } \delta_{i 1}=1, i=1 \text { e } \delta_{i 1}=0, i=2,3,4 \\
G_{s}\left(z, z_{n}\right), \sigma_{n}=\frac{1}{\sigma \sqrt{2 \pi}} \exp \left\{\frac{-\left(z-z_{n}\right)^{2}}{2 \sigma^{2}}\right\} \\
z_{n}=(n-1) 2 \sigma, \sigma=\frac{Z}{2 n_{\max } \sqrt{2 \ln 2}}
\end{array}
$$

$\mathrm{Na}$ expressão acima, $\boldsymbol{a}_{\boldsymbol{n}}$ representa a amplitude da guassiana $\boldsymbol{G}_{\boldsymbol{s}}$, o parâmetro $\boldsymbol{\sigma}$ é o desvio padrão dessa gaussiana, que varia com z. A vantagem na utilização do conjunto de funções gaussianas, é que para uma bicamada simétrica, é possível obter uma função analítica para $\mathrm{P}(\mathrm{q})$;

$$
P(q)=\sum_{n=1}^{4} a_{n} P(q, n), \text { onde } P(q, n)=\sqrt{2 \pi \sigma} \exp \left\{-\frac{\sigma^{2} q^{2}}{2} \cos \left(q z_{n}\right)\right\}
$$

Um exemplo do perfil obtido para P[q] é apresentado na figura 2. Io, na simulação de uma bicamada simétrica. Esse método de análise foi implementado no GFCx e foi utilizado para ajuste dos dados experimentais de espalhamento obtidos neste trabalho. Uma descrição mais detalhada sobre o tratamento dos dados experimentais e determinação dos parâmetros ajustáveis será apresentados na seção 4 do capítulo 3 .

\subsubsection{Propriedades elásticas das fases lamelares}

Como foi discutido na seção 2.5, a análise global das curvas de espalhamento de raios X pode fornecer não apenas os parâmetros estruturais que caracterizam a fase lamelar, mas também informações sobre as flutuações térmicas, caracterizadas pelo parâmetro de Caillé que relaciona as constantes elásticas $\bar{B}$ e K.

Encontram-se na literatura previsões para o comportamento do parâmetro de Caillé para duas situações particulares; i) para sistemas lamelares onde a interação predominante entre as bicamadas é de natureza eletrostática e ii) sistemas lamelares dominados por flutuações térmicas de ondulações, onde as forças predominantes são de natureza entrópica.

Barbara Bianca Gerbelli - Propriedades estruturais e elásticas de fases lamelares: O efeito da composição da membrana. 


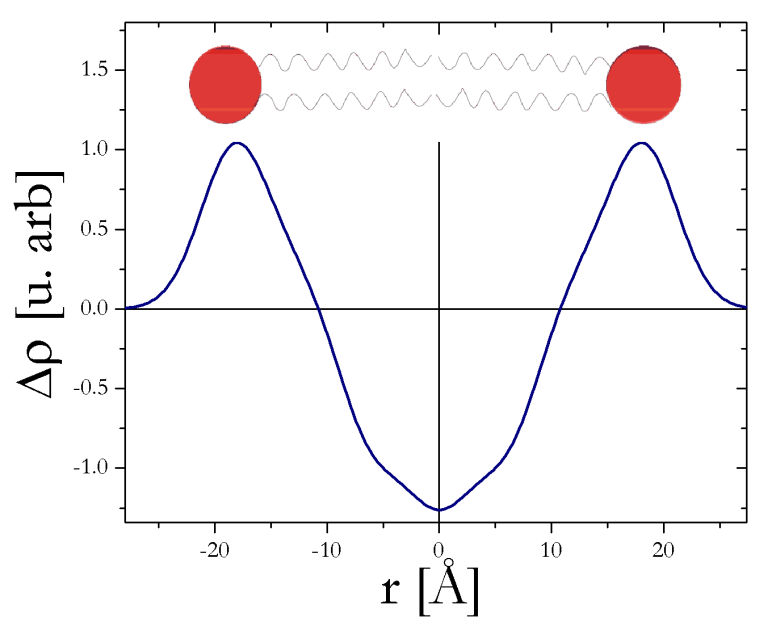

Figura 2. Iо.: Contraste de densidade eletrônico da bicamada obtida pelo modelo de 4 gaussianas .

A seguir serão apresentadas as previsões teóricas para o comportamento do parâmetro de Caillé para esses dois casos assim como resultados experimentais disponíveis na literatura. Veremos também, como a partir do parâmetro de Caillé podem ser determinadas a constante de rigidez da membrana e o módulo de compressão.

\section{Interações Eletrostáticas}

Sistemas lamelares compostos por surfactantes iônicos tais como, SDS (decil-sulfato de sódio), pentanol e água, ou SDS, hexanol e água, apresentam um grande domínio de diluição, com períodos lamelares atingindo valores da ordem de $130 \AA$ [46]. Nesses sistemas, a interação eletrostática entre as bicamadas é predominante em relação às outras interações devido à dupla camada na interface bicamada-água. As curvas de espalhamento de raios $\mathrm{X}$ observadas para esses sistemas lamelares mostram picos de espalhamento bastante estreitos, mesmo para diluições altas (fração volumétrica de água de $\mathbf{0 , 7 7}$ ). Esse fato experimental demostra que as flutuações térmicas não afetam o ordenamento da fase lamelar.

Para um sistema dominado por interações eletrostáticas [47], o comportamento do parâmetro de Caillé é dado por:

$$
\eta_{e l e}=\sqrt{\frac{\pi}{2} \frac{k_{B} T}{\kappa} \frac{L_{b}}{D}\left(1-\frac{\delta_{m}}{D}\right)^{3}},
$$

onde $\boldsymbol{L}_{b}$ é o comprimento de Bjerrum do solvente que no caso da água é da ordem $\approx 7$

Barbara Bianca Gerbelli - Propriedades estruturais e elásticas de fases lamelares: O efeito da composição da membrana. 
Å. Os dados obtidos e o ajuste feito a partir da equação 2.20 são representados na figura retirada da referência [47] (Figura 2. I I).

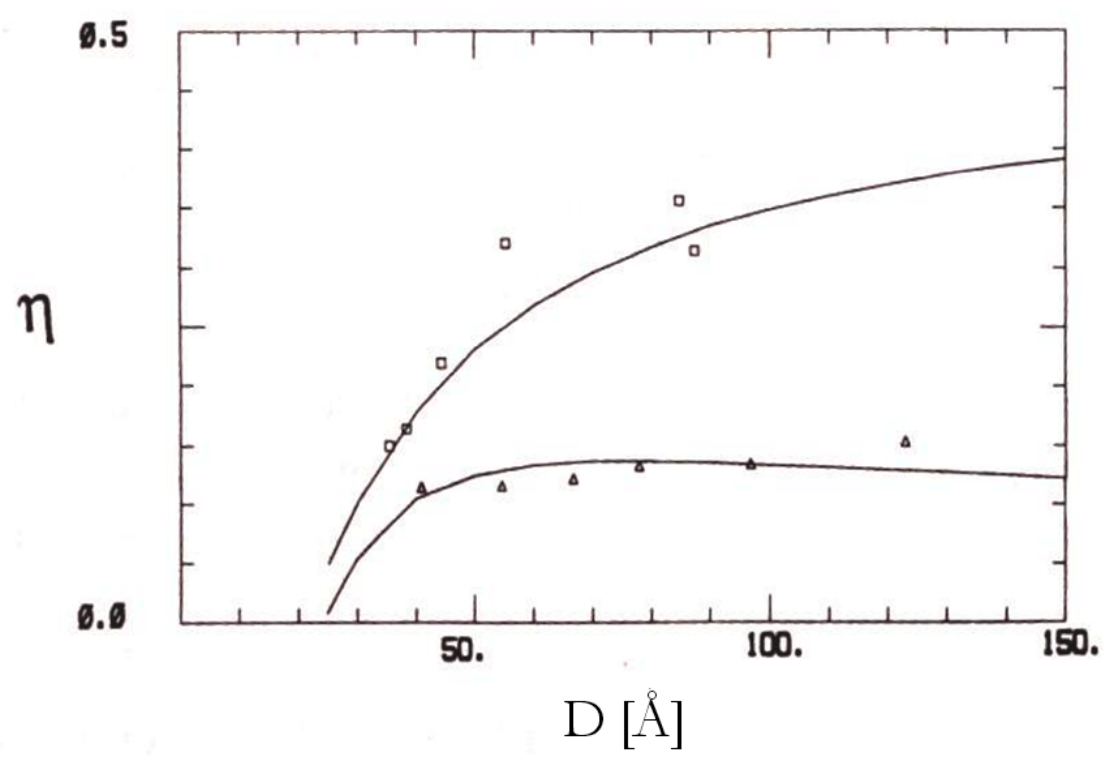

Figura 2. I r.: Parâmetro de Caillé vs. D, para sistemas lamelares compostos de moléculas ânionicas. (Figura extraída de [46])

Assim, a partir do ajuste dos dados experimentais de espalhamento, é possível obter o parâmetro de Caillé. Resultados experimentais obtidos para os dois sistemas citados acima mostram que o comportamento do parâmetro do parâmetro de Caillé é bem descrito pela equação 2.20. O ajuste da equação aos dados experimentais permite a determinação da constante de rigidez; que está entre 2 a $0,7 k_{B} T$ para o sistema com pentanol e entre 1 a $2 \boldsymbol{k}_{B} \boldsymbol{T}$ para o sistema com hexanol.

\section{Interações entrópicas}

Lipídios só formam fases lamelares para altas concentrações de surfactante, a partir de $60 \%$, e atingem período lamelar máximo em torno de $48 \AA$ A. A constante de rigidez das membranas foi estimada a partir da análise das flutuações térmicas em vesículas gigantes e é da ordem de $10-40 k_{B} \boldsymbol{T}$ [48]. Como a espessura da membrana é da ordem de $36-38$ Å, a distância de separação entre as bicamadas é muito pequena. Portanto, as flutuações térmicas são de pequena amplitude e a interação repulsiva de caráter estérico deve ter uma contribuição desprezível em relação às outras interações.

A adição de co-surfactantes, como um álcool, muda esse quadro de maneira extraordinária. A fase lamelar atinge periodicidade acima de $100 \AA$ [46] [47] [49], e o comportamento

Barbara Bianca Gerbelli - Propriedades estruturais e elásticas de fases lamelares: O efeito da composição da membrana. 
do parâmetro de Caillé segue uma lei de potências, compatível com a expressão obtida por Helfrich [40] ao considerar um sistema multilamelar dominado por interações de caráter estérico;

$$
\eta_{u n d}=\frac{4}{3}\left[1-\frac{\delta}{D}\right]^{2}
$$

Esse comportamento, ilustrado na figura (2.1 2) foi observado para inúmeros sistemas lamelares diretos (água como solvente ) e inversos (que utilizam óleo como solvente). No sistema lamelar composto por SDS-pentanol e água, as interações eletrostáticas podem ser blindadas pela adição de sal na água, e nesse caso, o comportamento do parâmetro de Caillé também é descrito pela expressão 2.2 I. (Figura 2.12)

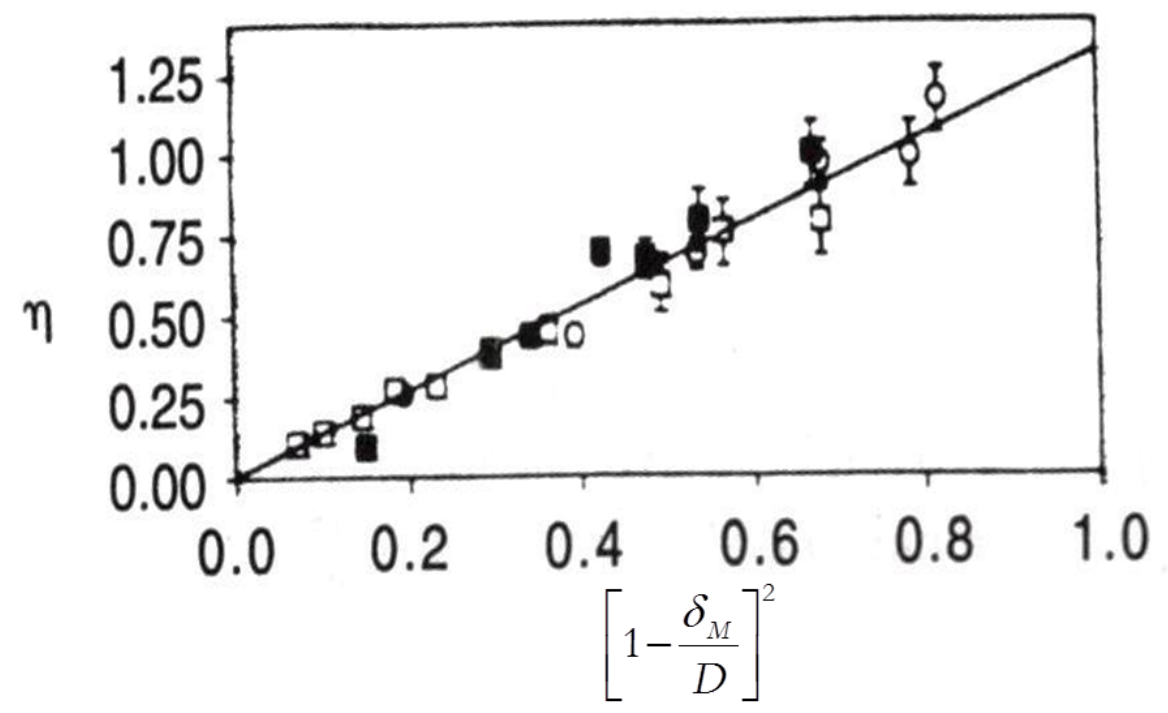

Figura 2. I 2.: (a) Valores experimentias obtidos para o parâmetro de Caillé em diferentes sistemas lamelares: DMPC-pentanol-água, SDS-pentanol-água+sal e SDSpentanol-água-óleo. A linha sólida corresponde ao ajuste dos dados experimentais à equação 2.2 I. (Extraída da referência de [49])

A incorporação do álcool como cosurfactante à membrana tem dois efeitos; promove o aumento da espessura da bicamada, e do período lamelar e reduz a constante de rigidez da membrana (figura 2.I 2) para valores da ordem de $\boldsymbol{k}_{B} \boldsymbol{T}$ [49].

\section{Módulo de compressibilidade para fases lamelares}

Podem ser definidas duas constantes de compressibilidade; B e $\overline{\boldsymbol{B}}$ que tem significado ligeiramente diferente; a constante $\overline{\boldsymbol{B}}$ (eq.2.22) representa o módulo de compressibilidade à potencial químico constante enquanto a constante $B$, que está presente na eq. 2.23

Barbara Bianca Gerbelli - Propriedades estruturais e elásticas de fases lamelares: O efeito da composição da membrana. 
representa a compressibilidade à concentração contante. B e $\bar{B}$ estão relacionadas pela expressão 2.2 ;

$$
\bar{B}=B-C \chi
$$

onde C é uma constante de acoplamento entre o deslocamento da camada e flutuações de concentração e $\chi$ é a compressibilidade osmótica, isto é a valor constante de $\mathrm{D}$. $\mathrm{O}$ significado dessas constantes é ilustrado na figura (2.13); a constante B está relacionada principalmente a elasticidade da membrana enquanto a constante $\bar{B}$ está relacionada às interações membrana-membrana e é dada por:

$$
\bar{B}=D \frac{\partial V^{2}}{\partial z^{2}}
$$

onde $\mathrm{V}$ é o potencial de interação entre as bicamadas.

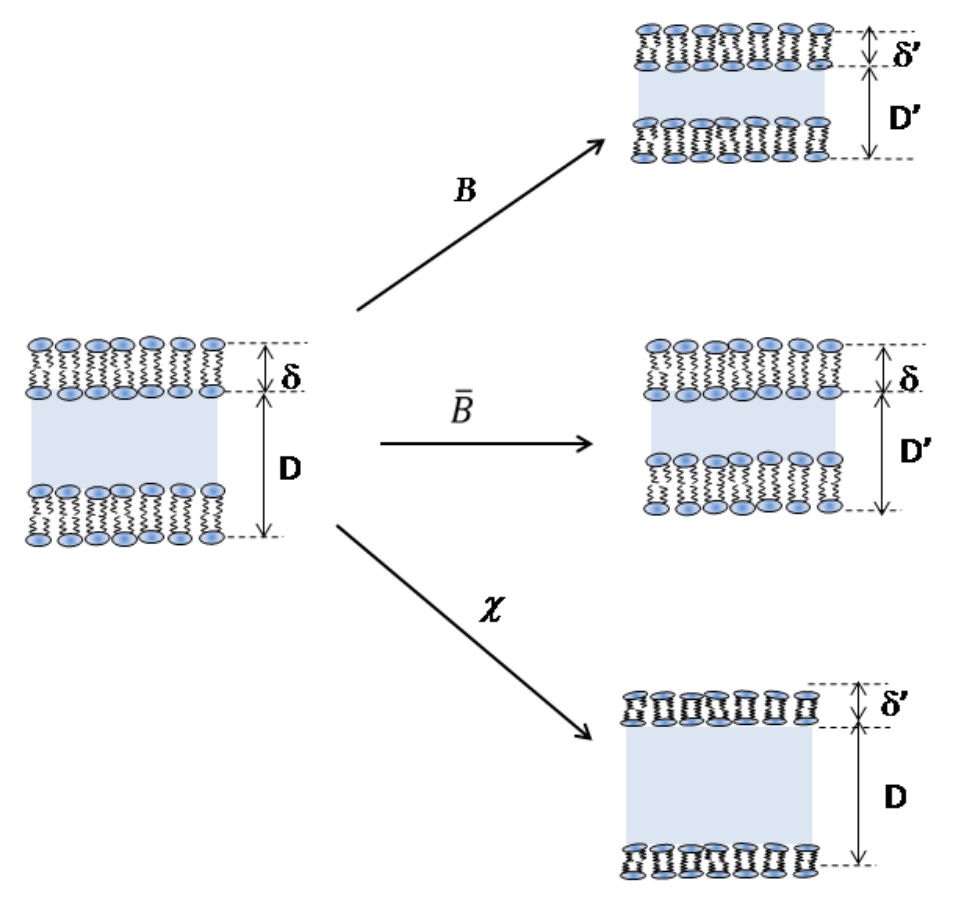

Figura 2.I 3.: Representação esquemática das deformações elásticas correspondentes às constantes elásticas $\mathrm{B}, \overline{\boldsymbol{B}}$ e $\chi$. A elasticidade da membrana está relacionada principalmente à $\bar{B}$ e $\chi$, enquanto $\mathrm{B}$ está relacionado ás interações entre as membranas.

Experimentalmente, a determinação do módulo de compressão é possível por meio de experimentos onde a fase lamelar é submetida á uma pressão osmótica controlada. Isso

Barbara Bianca Gerbelli - Propriedades estruturais e elásticas de fases lamelares: O efeito da composição da membrana. 
pode ser feito de duas formas; pela preparação da fase lamelar em uma solução, onde a pressão osmótica é controlada pela concentração de polímeros (PVP) [2 I] [43], ou por experimentos em que a fase lamelar está em contato com um reservatório à umidade controlada [3I].

Para bicamadas de DOPC (dioleoilfosfastidilcolina) a razão $\bar{B} / \boldsymbol{D}$ varia entre $\mathbf{1 0}-\mathbf{4 0} \times$ $10^{13} \mathrm{~J} / \mathrm{m}^{4}$, e decresce exponencialmente com o aumento da espessura da camada aquosa, independente da temperatura [3].

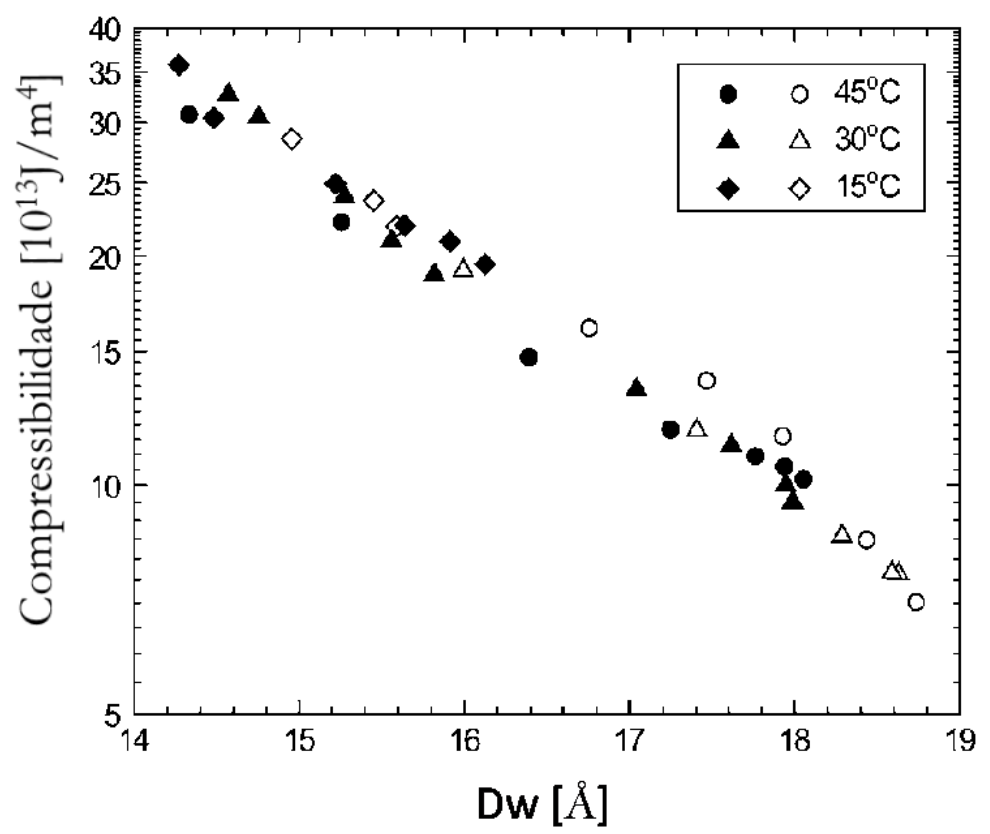

Figura 2. I 4.: Comportamento do módulo de compressibilidade (B/D) para a fase lamelar de DOPC, obtida por experimentos de difração de raios X em geometria de reflexão. (adaptada de [3])

Barbara Bianca Gerbelli - Propriedades estruturais e elásticas de fases lamelares: O efeito da composição da membrana. 
CAPÍTULO 3

MATERIAIS E MÉTODOS

\subsection{Preparação das Amostras}

\subsubsection{Composição das amostras}

Durante o trabalho realizado o surfactante utilizado para a preparação das amostras foi a lecitina de soja (Avati Polar ${ }^{\circledR}$ ) e um co-surfactante comercialmente conhecido como Simulsol ${ }^{\circledR}$ (Sigma Addrich). A lecitina é composta de uma mistura de fosfolipídios, majoritariamente de ácidos palmítico (DPPC); 63\% 18 : 2, 11, 4\% 18 : 1 e $5,7 \%$ de $18: 3^{\text {}}$. O segundo fosfolipídio predominante é o ácido miristoil, $14,9 \%$ e 3,7 de 16 : 1 de 16 : 0 . Levando em conta a proporção de fosfolipídios que constitui a lecitina calculou-se a massa molar média que correspondente à aproximadamente $776 \mathrm{~g} / \mathrm{mol} \mathrm{e}$ massa específica $\mathbf{1}, \mathbf{0 1} \mathrm{g} / \mathrm{cm}^{3}$.

O Simulsol é composto de uma mistura de moléculas monocaternárias etoxiladas. A cauda carbônica da molécula é funcionalizada a uma cadeia de polietilenoglicol com média de 10 monômeros. A massa molar média do Simulsol é de aproximadamente $278 \mathrm{~g} / \mathrm{mol}$ e massa específica de $1,02 \mathrm{~g} / \mathrm{cm}^{3}$.

\subsubsection{Processo de preparação e estabilização das amostras}

A homogeneidade da mistura do surfactante com o co-surfactante é obtida misturandose as quantidades desejadas de cada um dos componentes em um solvente orgânico (ciclo-

${ }^{\text {I}}$ Representado no gráfico são denotados $\mathrm{CN}$ :M carbonos que constitui a cadeia hidrofóbica e o número de insaturações dessas mesmas respectivamente

Barbara Bianca Gerbelli - Propriedades estruturais e elásticas de fases lamelares: O efeito da composição da membrana. 


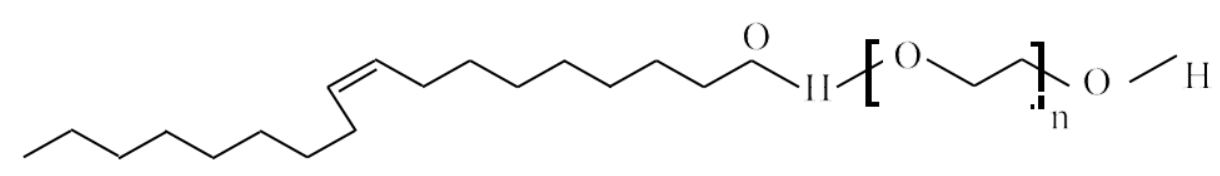

(a)

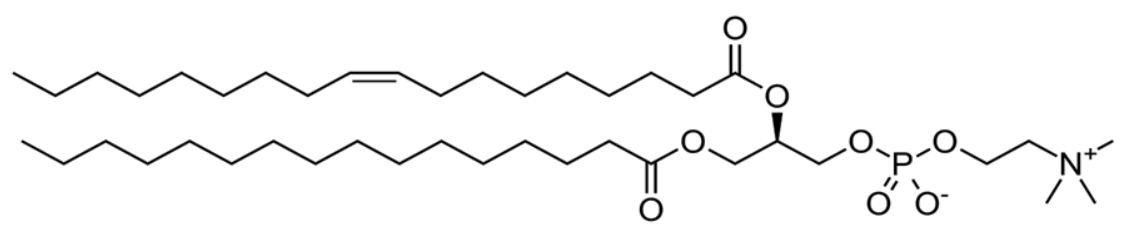

(b)

Figura 3. I.: Fórmula estrutural química (a) cadeia monocaternária + cadeia polimérica e (b) da fosfatidilcolina

hexano) numa proporção de $100 \mathrm{~g}$ de lipídio (Simulsol+lecitina) preparado para $\mathbf{1 , 5}$ $\mathrm{ml}$ de solvente. A mistura é submetida a ciclos de sonicação até que a solução fique homogênea. Para evaporação do solvente a solução é distribuída em tubos tipo "ependorf” que são colocados em uma estufa á vácuo por períodos que podem variar de $\mathbf{2 4}$ horas á $\mathbf{7 2}$ horas. Para cada composição de membrana estudada foi explorada uma linha de diluição, variando-se a fração volumétrica de lipídio (ou água) dada por:

$$
\phi_{i}=\frac{m_{i}}{\rho_{i} V_{T}}
$$

onde $\phi_{i}$ é a fração volumétrica do constituinte, $\boldsymbol{m}_{\boldsymbol{i}}$ é a massa de água ou do lipídio usado na preparação da amostra, $\boldsymbol{\rho}_{\boldsymbol{i}}$ é a massa específica do composto e $\boldsymbol{V}_{\boldsymbol{T}}$ é o volume total. Foi adotado o protocolo de preparação das amostras, com um volume total $V_{T}=100 \mu L$, e a pesagem da massa de lipídios realizada em uma balança analítica com precisão de $\mathbf{0 , 1} \mathrm{mg}$ à temperatura ambiente. Os componentes foram armazenados em tubos tipo "ependorf" e mantidos em temperatura da ordem de $5^{\circ} \mathrm{C}$. Para a homogeneização das amostras foram realizados ciclos alternados de centrifugação de $\mathbf{3 0}$ minutos de "cabeça para cima" e depois de "cabeça para baixo" juntamente com variações de temperaturas da ordem de $30^{\circ}$. A velocidade de centrifugação utilizada foi de $600 \mathrm{rmp}$ (rotações por minuto), onde esse processo foi realizado pelo menos 4 vezes por dia com intervalos de $\mathbf{2}$ dias de descanso após cada ciclo durante 1 mês. $\mathrm{O}$ número total de amostras que foram formuladas para a realização deste trabalho foi de mais de 205 .

Barbara Bianca Gerbelli - Propriedades estruturais e elásticas de fases lamelares: O efeito da composição da membrana. 


\subsection{Experimentos de SAXS e WAXS}

Para a técnica de Espalhamento de Raios X a Baixo Ângulo (SAXS) e Espalhamento de Raios X a Alto Ângulo (WAXS) foi utilizado uma máquina convencional Nanostar Bruker instalada no Laboratório de Cristalografia do Instituto de Física. Nestes experimentos foram utilizados capilares de vidro de $1,5 \mathrm{~mm}$ de diâmetro onde por centrifugação as amostras eram depositadas no fundo do capilar. Esse procedimento resulta em uma orientação aleatória das bicamadas no interior do capilar. Além da utilização da máquina NanoStar experimentos em luz sincrontron foram realizados (linha SWING - SOLEIL França). Esses últimos experimentos foram realizados por um aluno doutoramento [5o], porém o tratamento dos dados bem como as análises foram realizados pela autora.

\subsubsection{Fontes convencionais - Nanostar}

Cerca de $\mathbf{7 0 \%}$ dos experimentos realizou-se no equipamento o Nanostar-Bruker (Figura 3.2). A máquina é utilizada para estudos estruturais na faixa de 0,003 á $0,85 \AA^{-1}$. O feixe é produzido por uma fonte convencional com a radiação pela desaceleração dos elétrons em um ânodo de $\mathrm{Cu}$, com duas linhas característica desse material; $\boldsymbol{K}_{\boldsymbol{\alpha}}$ e $\boldsymbol{K}_{\boldsymbol{\beta}}$.Um monocromador de silício 100 seleciona somente a radiação da $K_{\alpha 1}$ e $K_{\alpha 2}$ para formar o feixe de fótons que interage com a amostra, e o comprimento de onda médio das duas linhas $K_{\alpha}$ é de $1,5418 \AA^{-1}$. Além do monocromador, a ótica do equipamento consiste de um sistema de espelhos de Gobel e $\mathbf{3}$ fendas que geram um feixe de $\mathrm{Imm}$ de diâmetro e o fluxo do feixe incidente é de $2 \times 10^{6}$ fótons/s. Foi utilizado um detector a gás Hi-Star $1024 \times 1024$ pixels, cada um de $100 \mu \times 100 \mu^{2}$ e um "beamstopper" semi-transparente. As imagens $2 \mathrm{D}$ obtidas sofrem correções espaciais e de distorção e para isso utiliza-se um software fornecido pelo fabricante.

A distância média amostra-detector utilizada para a técnica de SAXS foi de $65 \mathrm{~cm}$ que nos permitia ter acesso a valores de $q$ no espaço recíproco no intervalo de $\mathbf{0 , 0 0 4 < \mathrm { q } <}$ $0,35 \AA^{-1}$ e para WAXS a distância utilizada foi de aproximandamente $6 \mathrm{~cm}$ varrendo, no espaço recíproco, o intervalo de $0,01<\mathrm{q}<0,85 \AA^{-1}$.

$\mathrm{Na}$ realização de experimentos de SAXS utilizou-se um porta amostra que acomodava até 15 capilares de vidro à temperatura ambiente de cerca de $22^{\circ}$. Nos experimentos de WAXS usou-se o mesmo porta-amostra, ligado a um banho térmico com circulação de água pelo suporte para o controle da temperatura abaixo e acima da temperatura ambiente.

${ }^{2}$ Essas informações foram obtidas através da pagina do Grupo de Cristalografia do IFUSP disponível em: http://web.if.usp.br/cristal/node/ ro3

Barbara Bianca Gerbelli - Propriedades estruturais e elásticas de fases lamelares: O efeito da composição da membrana. 


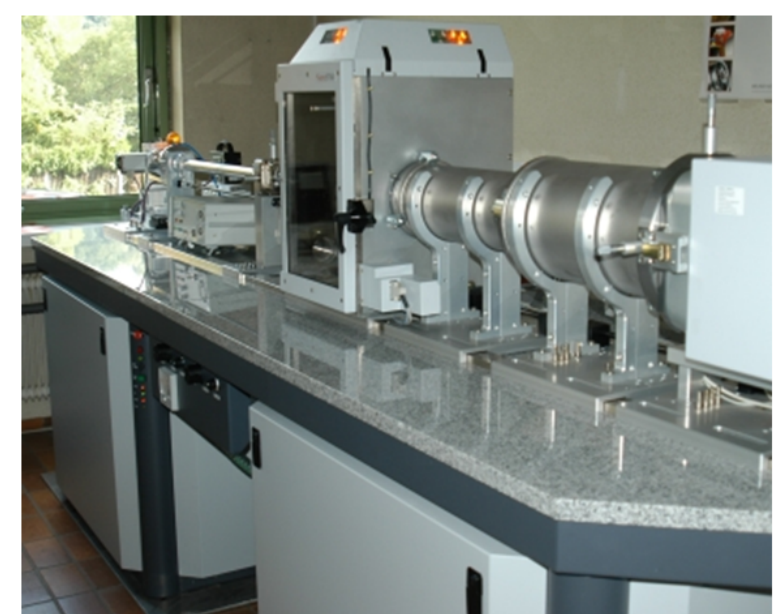

(a)

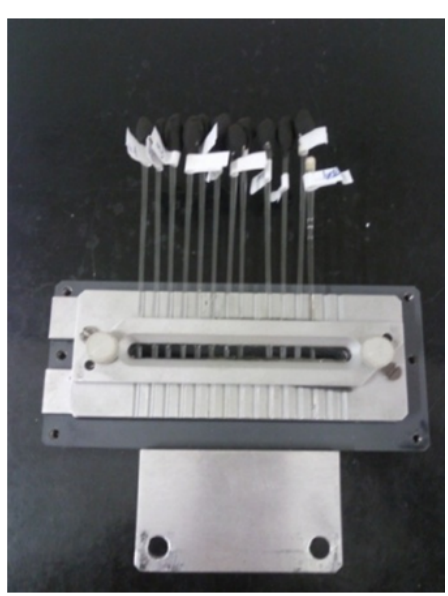

(b)

Figura 3.2.: (a) - Fonte convencional de raios X utilizada - Nanostar. (b) porta amostra no desenvolvimento desse trabalho.

O sistema de operação do Nanostar permite encontrar a posição ótima para realizarmos aquisição de cada amostra fazendo uma varredura nas coordenadas de X e Y de cada capilar. A realização da varredura permite ter uma idéia da transmitância das amostras, possibilitando a determinação do tempo de exposição para cada uma individualmente; esse tempo varia de $\mathbf{2 0}$ à $\mathbf{6 0}$ minutos dependendo da concentração de água de cada amostra.

$\mathrm{O}$ controle das aquisições foi realizado pelo próprio programa do Nanostar chamado SAXS - BRUKER, onde em cada aquisição feita, são geradas imagens bidimensionais de formato ".gfrm". Para análise desses arquivos foi utilizado o programa SUPERSAXS [5 I] que permite, desde a subtração de fundo e parasita, até a redução dessas imagens em curvas unidimensionais $(\mathrm{I}(\mathrm{q})$ ) com as respectivas incertezas dos dados experimentais.

\subsection{Métodos de tratamento e ajustes das curvas experimentais de SAXS}

\subsubsection{Tratamento dos dados}

Antes de realizar as aquisições dos dados é necessário seguir o procedimento de calibração padrão para determinar com precisão a distância amostra-detector. Como padrão

Barbara Bianca Gerbelli - Propriedades estruturais e elásticas de fases lamelares: O efeito da composição da membrana. 
utilizou-se o behenato de prata (BeAg) obtendo uma distância média de $65 \mathrm{~cm}$.

Para o tratamento dos dados deve-se levar em conta todas as contribuições possíveis para I(q). No caso das bicamadas de lipídios, quando preparadas estão imersas em um solvente (água) e temos que considerar que este meio deve contribuir para o espalhamento final que observamos. Além do solvente as membranas estão contidas dentro do capilar de vidro que também contribui para o espalhamento final. Levando-se em conta do que o espalhamento possui de 3 contribuíções, a análise dos dados passa por três momentos.

Para obter apenas o espalhamento da fase lamelar são feitas aquisições de dados para um capilar de vidro vazio e outro capilar preenchido com o solvente, e os espalhamentos gerados por ambos são subtraídos da curva de espalhamento obtida para a fase lamelar no capilar de vidro. Após este processo obtêm-se um sinal I(q) que é chamado de $\boldsymbol{I}(\boldsymbol{q})_{\text {tratada }}$ (equação 3.2).

$I_{\text {tratada }}=\left[\left(\frac{I_{\text {amostra }}(q)}{\Phi_{s} T_{s} t_{s}}-\frac{I_{\text {back }}(q)}{\Phi_{b} T_{b} t_{b}}-\frac{I_{\text {ruido }}(q)}{t_{\text {ruido }}\left(\Phi_{s} T_{s}-\Phi_{b} T_{b} t_{b}\right)}\right) \frac{1}{I_{\text {sombra }}(q)}\right] \frac{\frac{d \sum}{d \Omega_{a g u a, 20^{\circ}}}}{I(0)_{a g u a, 20^{\circ}}},(3.2)$

onde, $\boldsymbol{I}_{\text {amostra }}$ corresponde ao espalhamento do conjunto amostra + solvente+capilar; $\boldsymbol{I}_{\text {back }}$ corresponde ao espalhamento do solvente + capilar ("background").

As intensidades $\boldsymbol{I}_{\text {ruido }}, \boldsymbol{I}_{\text {sombra }}$ são pré-aquisições que correspondem a corrente de fundo do equipamento e a sombra do feixe direto incidem no "beamstopper", respectivamente. $\Phi_{i}$ representa o fluxo do feixe incidente onde os índices $s$ e $b$ indicam a amostra e o parasita, respectivamente. As transmitâncias são representadas pelas letras $T$. Para o termo $\frac{d \sum / d \Omega_{a g u a, 20^{\circ}}}{I(\mathbf{0})_{a g u a, 20^{\circ}}}$ utilizamos como um padrão secundário a água, por ser um padrão cujo espalhamento não depende do ângulo de espalhamento na faixa angular de interesse. À temperatura de $20^{\circ} \mathrm{o}$ valor para a seção de choque da água é de $0,01632 \mathrm{~cm}^{-1}$ [52].

O cálculo da transmitância da amostra é dada pela equação 3.3

$$
T_{S}=\frac{I_{S+G C}-T_{G C} I_{G C}-I_{\text {ruido }}}{I+G C}
$$

onde $\boldsymbol{I}_{\boldsymbol{S}+G C}$ são aquisições com um centro espalhador (carvão vítreo) colocado na frente da amostra, no caso do Nanostar. $\boldsymbol{I}_{G C}$ corresponde a uma aquisição em um intervalo de tempo de 30 minutos á I hora, a transmitância do carvão vítreo é obtida juntamente nesse procedimento. $\mathrm{O}$ cálculo da incerteza é dado pela equação 3.4

Barbara Bianca Gerbelli - Propriedades estruturais e elásticas de fases lamelares: O efeito da composição da membrana. 


$$
\begin{gathered}
\sigma_{\text {tratado }}(q)=\left[\left(\frac{\sigma_{i}^{2}(q)}{I_{\text {sombra }}^{2}(q)}\right)+\left(\frac{I_{\text {amostra }}(q)}{\Phi_{s} T_{s} t_{s}}\right)^{2} \frac{\sigma_{\text {sombra }}{ }^{2}(q)}{I_{\text {sombra }}^{2}(q)}\right]^{1 / 2} \\
\sigma_{1}(q)=\sigma_{\text {amostra }}^{2}(q)+\sigma_{\text {back }}^{2}(q)+\sigma_{\text {ruido }}^{2}(q)\left[\frac{1}{\Phi_{s} T_{s}}-\frac{1}{\Phi_{b} T b}\right]
\end{gathered}
$$

onde $\sigma_{i}$ representa um erro estatístico para cada ponto com os mesmos índices utilizados para descrever as intensidades. O termo $\sigma_{\text {sombra }}$ representa um erro estimado para o erro correspondente à contribuição da sombra atrás do "beamstopper" [52]).

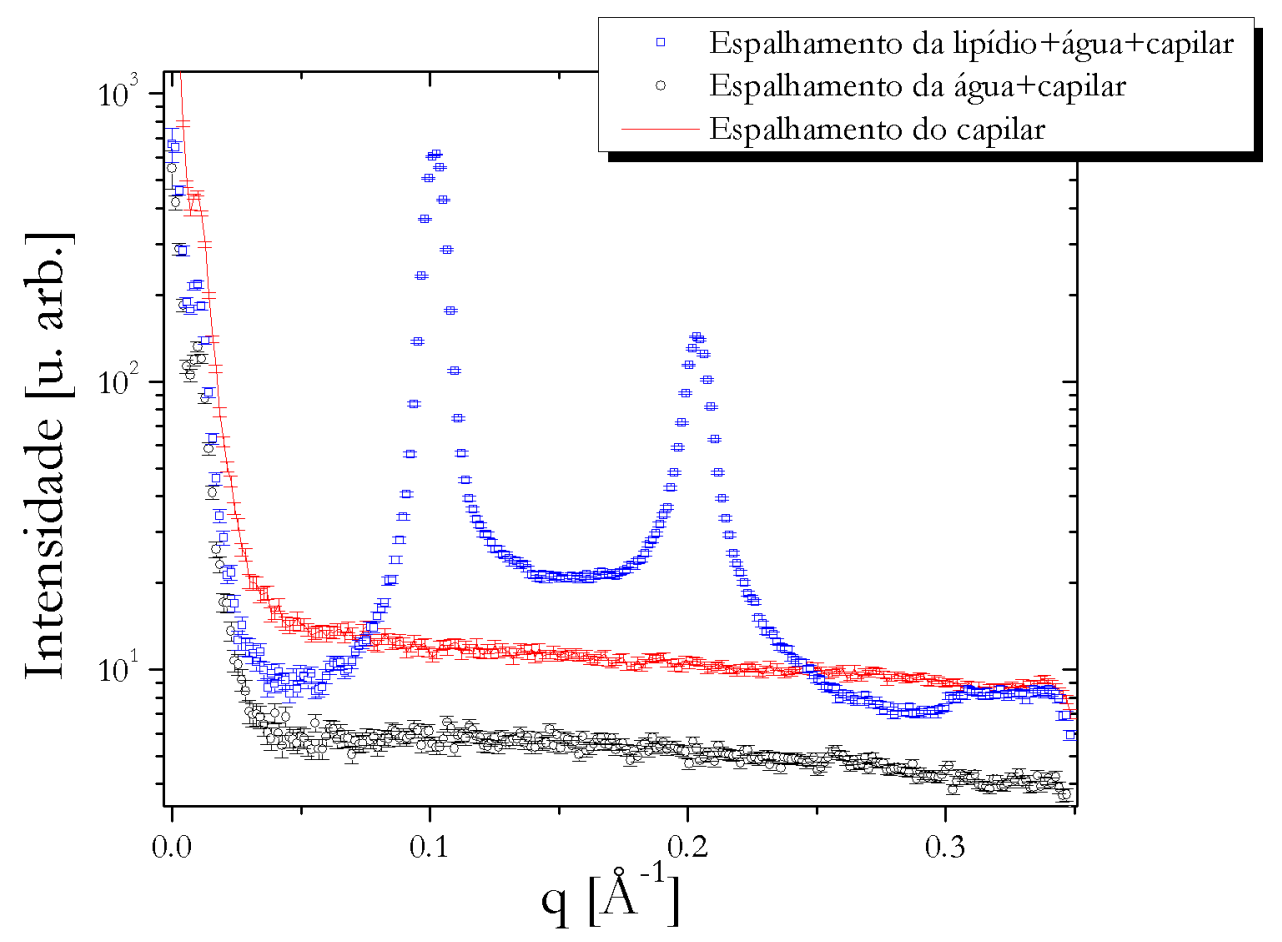

Figura 3.3.: Exemplo de curva de SAXS bruto e as contribuições do espalhamento de água e capilar vazio.

Barbara Bianca Gerbelli - Propriedades estruturais e elásticas de fases lamelares: O efeito da composição da membrana. 


\subsubsection{Ajuste das curvas de espalhamento}

Para uma fase lamelar a intensidade espalhada pode ser descrita como:

$$
I(q)=\frac{S(q)\left|P(q)^{2}\right|}{q^{2}}
$$

onde $q$ é o módulo do vetor de espalhamento de raios $\mathrm{X}, \mathrm{S}(\mathrm{q})$ representa a função do fator de estrutura, $\mathrm{P}(\mathrm{q})$ o fator de forma do sistema. O fator de forma depende somente do espalhamento gerado por uma única bicamada, enquanto o fator de estrutura deve corresponder ao espalhamento produzido por um conjunto de bicamadas, periodicamente espaçadas com orientação aleatória. Para descrever o fator de forma foram testados dois modelos chamados d: 3 degraus e deconvolução de gaussianas (4 gaussianas para este trabalho).

\section{Fator de estrutura}

Como discutido no Capítulo 3 os parâmetros que descrevem a estrutura e a flexibilidade da membrana podem ser determinados pelos parâmetros determindos no ajuste das curvas experimentais entre eles os principais são: periodicidade lamelar, número de camadas correlatas e parâmetro de Caillé.

Nesta seção tem-se como objetivo ilustrar a influencia de cada um dos parâmetros descritos acima na curva de espalhamento I(q) resultante. A periodicidade lamelar pode ser determinada pela lei de Bragg, o comportamente de $\boldsymbol{D}$ é inversamente proporcional ao vetor de onda, pode ter-se então uma ideia de como a periodicidade lamelar modifica I(q). $\mathrm{Na}$ figura 3.4 observa-se que os picos de espalhamento deslocam-se para valores menores de $q$ com o aumento da hidratação da fase lamelar.

O efeito da variação do parâmetro de Caillé e do número de camadas correlatas é ilustrado na figura 3.5 e $3.5(\mathrm{~b})$. O decrescimento de qualquer um desses parâmetros produz alargamento nos picos, e também a diminuição no número de picos observáveis.

Barbara Bianca Gerbelli - Propriedades estruturais e elásticas de fases lamelares: O efeito da composição da membrana. 


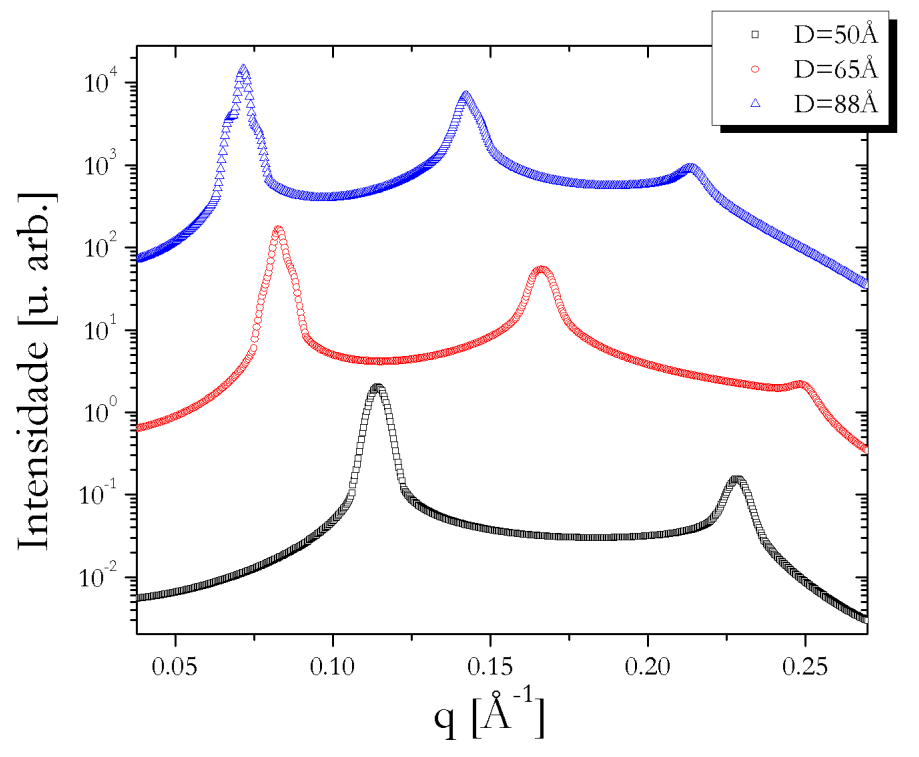

Figura 3.4.: Simulação de curvas de espalhamento variando a periodicidade lamelar, com $\mathrm{N}=50$ e $\boldsymbol{\eta}=0,1$ composição da membrana. 


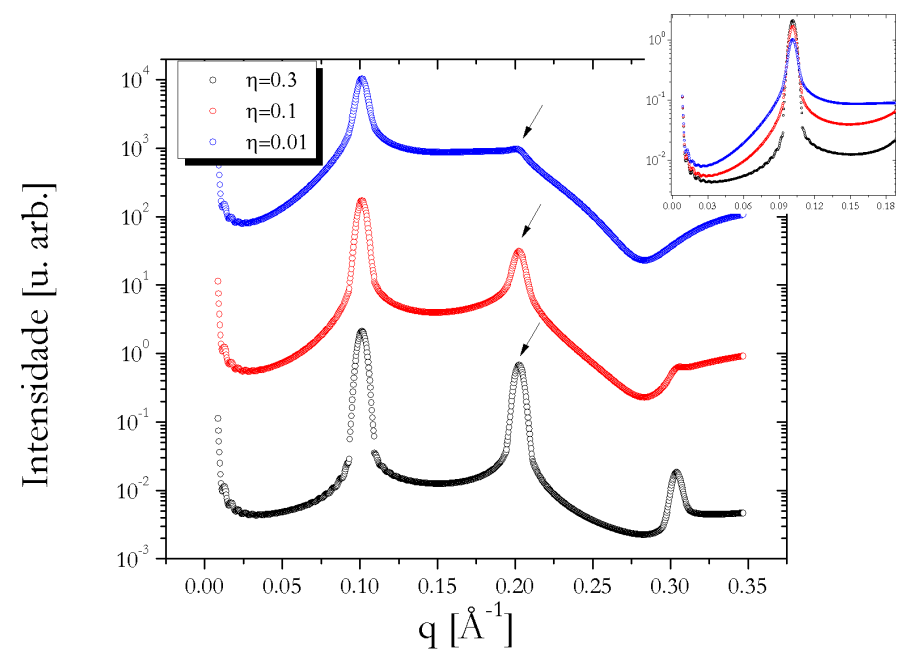

(a)

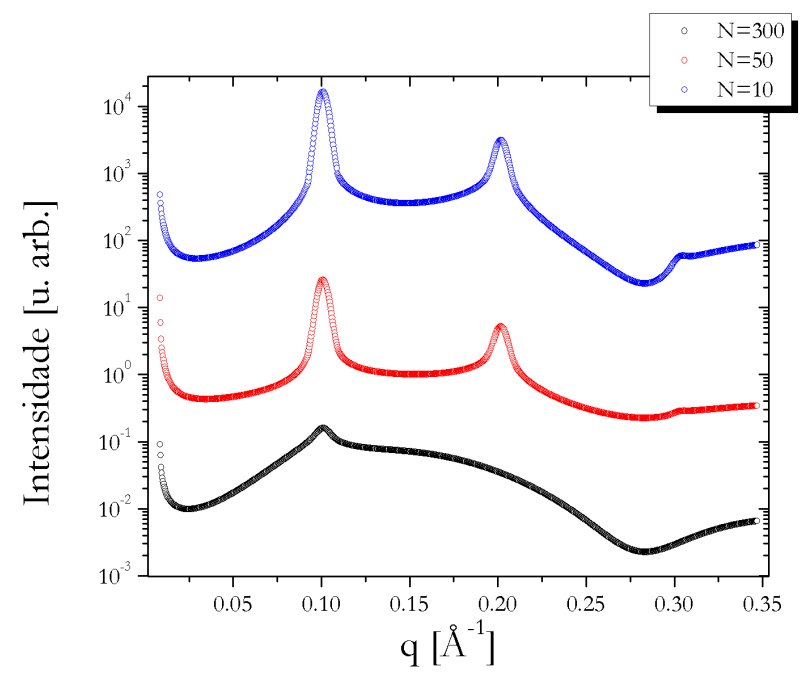

(b)

Figura 3.5.: Simulação de curvas de espalhamento variando (a) $\boldsymbol{\eta}$ e (b) $\mathrm{N}$ com $\mathrm{D}=\mathbf{6 5} \AA$.

Barbara Bianca Gerbelli - Propriedades estruturais e elásticas de fases lamelares: O efeito da composição da membrana. 


\section{Fator de forma}

O fator de forma descreve a densidade do contraste eletrônico da bicamada como discutido Capítulo 3. A diferença entre os modelos é que o modelo de 3 de graus representa uma mudança no contraste eletrônico de forma abrupta não apresentando uma descrição precisa da interface membrana-camada aquosa. Utilizando o modelo de deconvolução de gaussianas a variação do contraste eletrônico é descrita de maneira mais suave. (Figura 3.6)

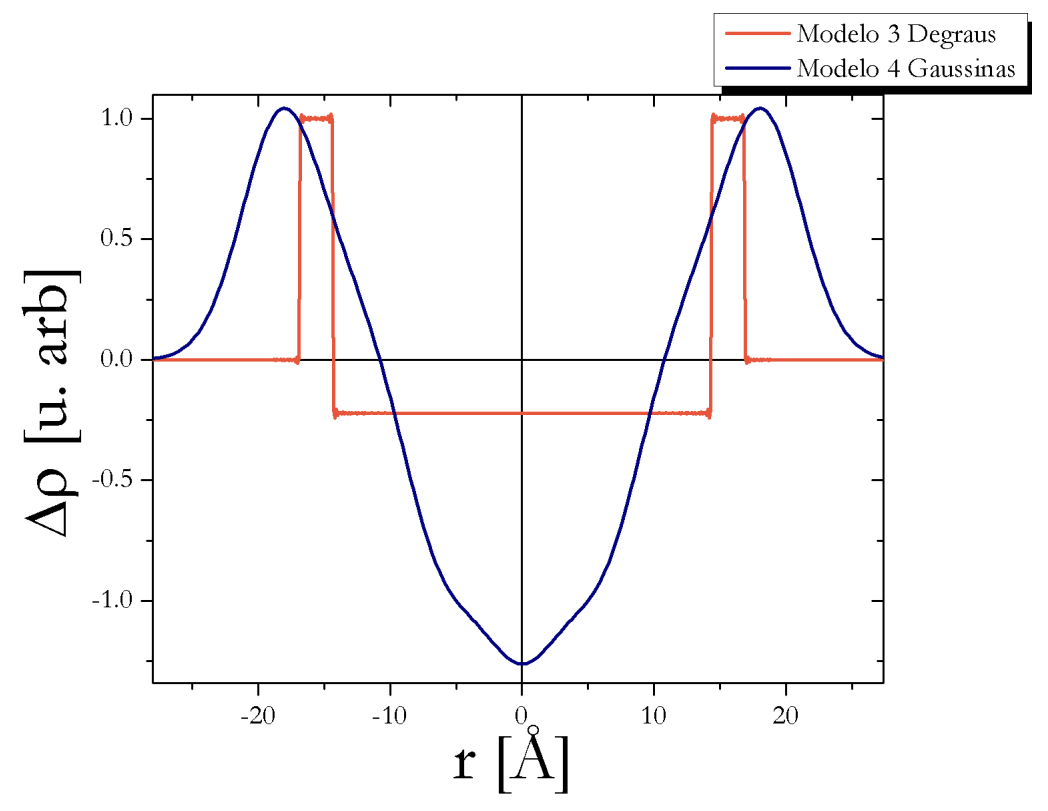

Figura 3.6.: Representação esquemática do contraste de densidade eletrônico da bicamada obtida pelo modelo de 3 degraus e pelo modelo de 4 gaussianas. Nesse modelo a parte positiva do contraste se refere a cabeça polar e negativa as caldas carbônicas. Contraste de densidade eletrônico da bicamada obtida.

$\mathrm{Na}$ figura 3.7 é possível comparar o ajuste utilizando os dois modelos de fator de forma para descrever a bicamada discutidos na seção 2.4.2. Na região de baixo ângulo (q pequenos) ambos os modelos fornecem um ajuste satisfatório, porém, à medida que $q$ aumenta, o modelo de 3 de graus já não descreve de maneira adequada os dados experimentais.

No Capítulo 4, a análise dos dados que serão apresentados fará uso do modelo de 4 gaussianas para o fator de forma da bicamada.

\subsubsection{Determinação da espessura da bicamada}

$\mathrm{O}$ ajuste dos dados experimentais de espalhamento fornece a periodicidade lamelar e o perfil de densidade eletrônica que descreve a bicamada. O pico representa a posição das

Barbara Bianca Gerbelli - Propriedades estruturais e elásticas de fases lamelares: O efeito da composição da membrana. 


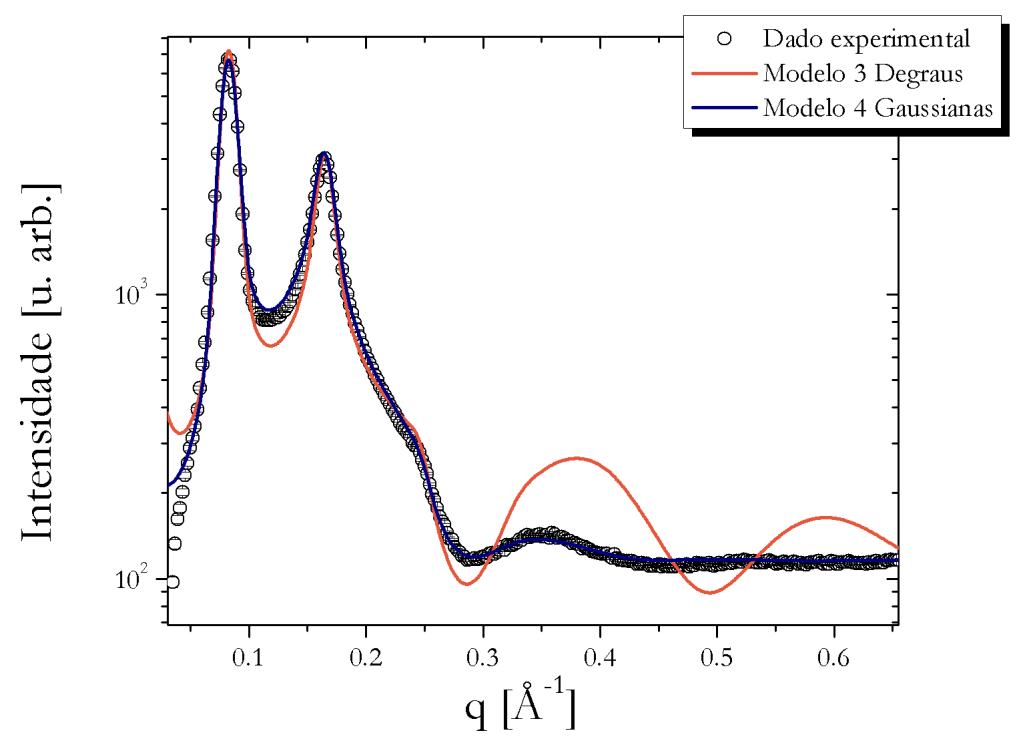

Figura 3.7.: Ajuste da curva experimental com os dois modelos para o fator de forma.

cabeças polares e no centro ( $\mathrm{r}=\mathrm{o}$ na figura 3.8 ) corresponde ao meio da bicamada. $\mathrm{O}$ contraste de densidade eletrônico da região é descrito por uma função gaussiana e tomando-se a distância do centro da bicamada até o ponto correspondente a meia altura da gaussiana, tem-se a metade da espessura da bicamada. Nesse ponto pode-se ter uma boa aproximação da interface dos grupos polares da molécula da bicamada e as moléculas de água.

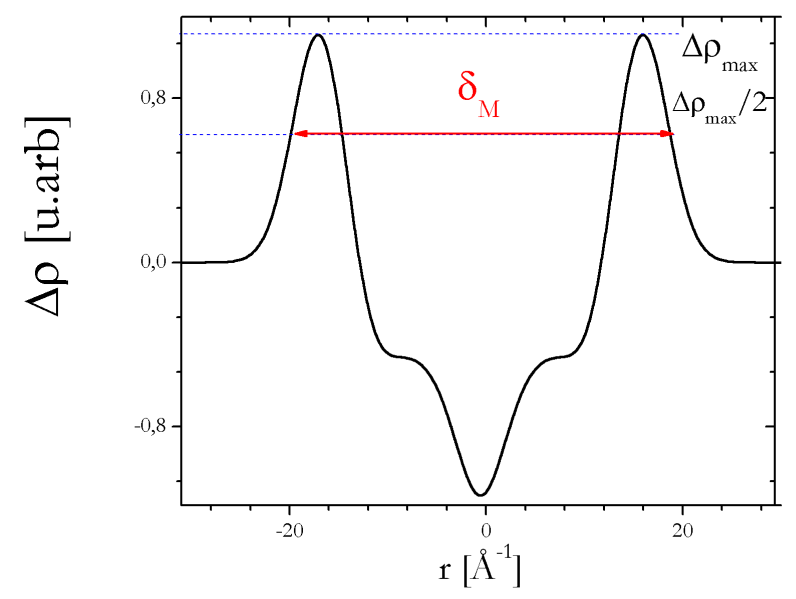

Figura 3.8.: Perfil de contraste de densidade eletrônico para uma membrana lipídica.

Barbara Bianca Gerbelli - Propriedades estruturais e elásticas de fases lamelares: O efeito da composição da membrana. 


\subsection{Experimentos de GISAXS}

Uma das principais contribuições deste trabalho foi o desenvolvimento da implementação da técnica de Incidência Razante de Raios X á Baixo Ângulo (GISAXS) realizando adaptações no equipamento NanoStar-Bruker. A primeira mudança foi na câmara do equipamento que passou a funcionar em pressão atmosférica. Para isso foi necessário introduzir uma janela entre a câmara e a fonte de raios X, e uma segunda montada em um cone entre a câmara e o detector (Figura 3.9 a e b).

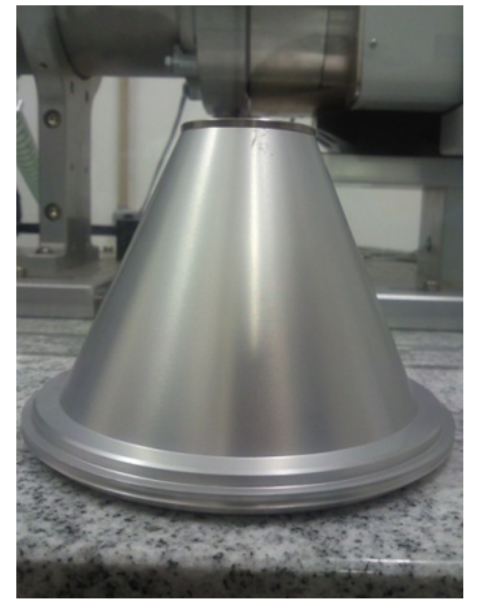

(a)

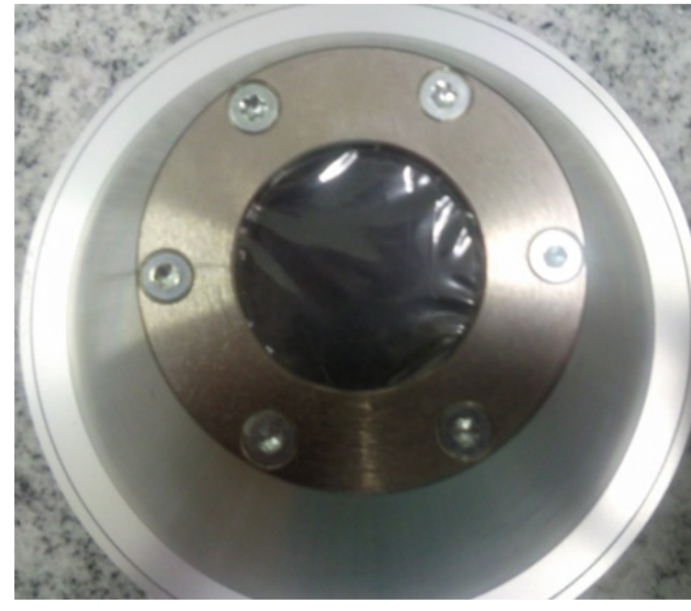

(b)

Figura 3.9.: Imagens do "cone" de adaptação entre a câmara e o detector vista (a) lateral e (b) superior.

Adaptações mecânicas foram desenvolvidas para conseguir acomodar o goniômetro WT-90 (Micos) à câmara de raios X, que são descritos com maiores detalhes no apêndice A.

Para estudar o processo cinético de hidratação/desidratação da fase lamelar foi desenvolvido um porta-amostra, com duas janelas de polímero transparente ao raios $\mathrm{X}$ permitindo a passagem do feixe de raios $\mathrm{X}$. No interior do porta-amostra é colocada uma lâmina de vidro, sobre o qual a fase lamelar é depositada e cisalhada unidirecionalmente. Esse processo leva à orientação das bicamadas paralelas à superfície da lâmina de vidro.

O porta-amostra está conectado à um umidificador e o fluxo de vapor de água pode ser controlado externamente. A umidade relativa no interior do porta-amostra é medida por

Barbara Bianca Gerbelli - Propriedades estruturais e elásticas de fases lamelares: O efeito da composição da membrana. 
um sensor de umidade, que está posicionado próximo à lâmina de vidro.

O porta-amostra é montado sobre uma mesa ótica que permite a rotação do conjunto em torno de um eixo vertical, para que as janelas do porta-amostra sejam alinhadas com o feixe. Esse conjunto é então montado sobre um goniômetro, que é usado para variar o ângulo de incidência do feixe de raios X sobre a lâmina contendo o filme. Para o estudo das fases lamelares os ângulos de incidência utilizados estão entre $\mathbf{0 , 5}$ a 2, $\mathbf{5}^{\mathbf{o}}$ (Figura 3. Io).

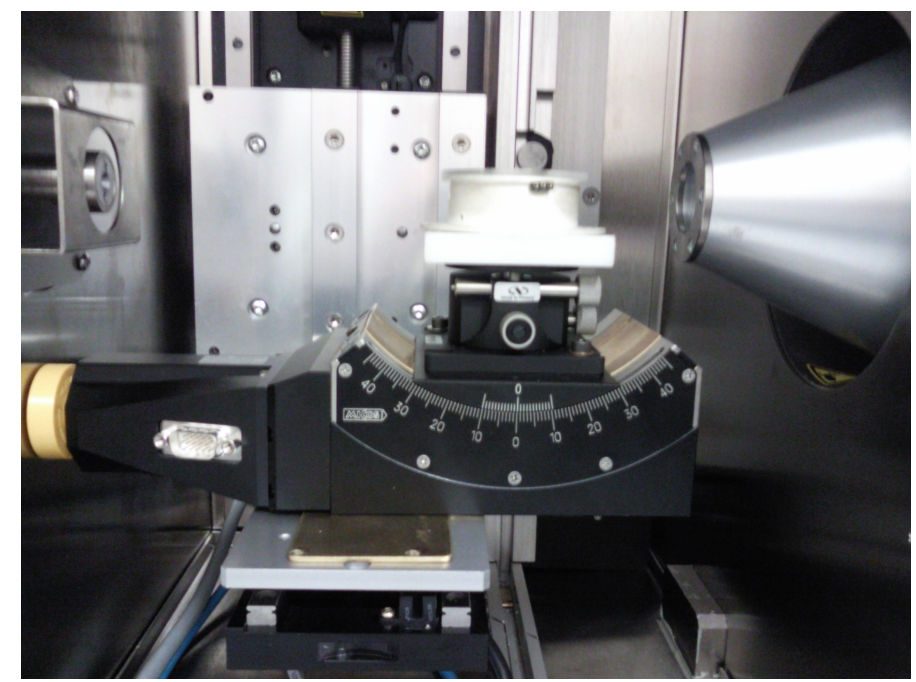

Figura 3. Io.: Porta amostra com controle de umidade instalado sobre o goniômetro na câmara do NanoStar.

Para iniciar os experimentos necessita-se uma primeira varredura em X-Y para estabelecer os limites da janela para acesso ao feixe incidente. Em seguida uma varredura mais fina no eixo Y é feita para determinar o plano da amostra, na Figura 3. I I tem-se um gráfico da intensidade do feixe em função da coordenada $\mathrm{Y}$ do porta amostra. $\mathrm{O}$ plano da amostra é escolhido, tomando o de y para qual a intensidade do feixe cai para a metade do valor máximo.

Para a calibração da distância amostra-detector foi preparado um filme de behenato de prata (BeAg) sobre uma lâmina de vidro semelhante à que é usada como substrato para a fase lamelar. Para encontrar o ângulo de Bragg foram feitas aquisições de $30 \mathrm{~s}$ variando o ângulo de incidência com passos de $\mathbf{0}, \mathbf{2}^{\boldsymbol{o}}$ na inclinação do plano da amostra.

No detector são observados bandas circulares, concêntricas, que correspondem aos picos de espalhamento do BeAg Figura 3 . I 2 cujos valores de $\boldsymbol{q}_{\boldsymbol{e x p}}$ são conhecidos. A integração da imagem do detector usando o valor aproximado para a distância detector-amostra $\left(\boldsymbol{L}_{a p}\right)$ fornece valores de $\boldsymbol{q}_{\boldsymbol{m e d}}$. A distância experimental amostra-detector $\left(\boldsymbol{L}_{\text {exp }}\right)$ é deter-

Barbara Bianca Gerbelli - Propriedades estruturais e elásticas de fases lamelares: O efeito da composição da membrana. 


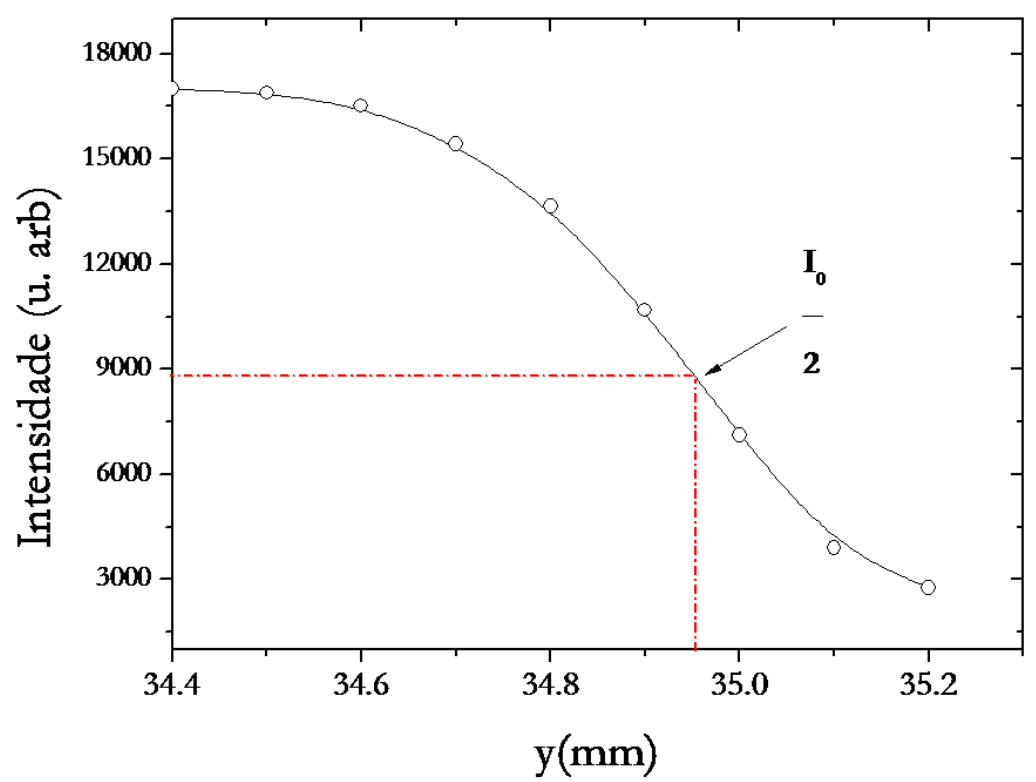

Figura 3. I I.: Intensidade pela posição y, para encontrar o plano da amostra.

minada usando a equação 3.6. Esse procedimento é ilustrado na figura 3.1 3

$$
L_{e x p}=L_{a p} \frac{q_{e s p}}{q_{m e d}}
$$

Barbara Bianca Gerbelli - Propriedades estruturais e elásticas de fases lamelares: O efeito da composição da membrana. 

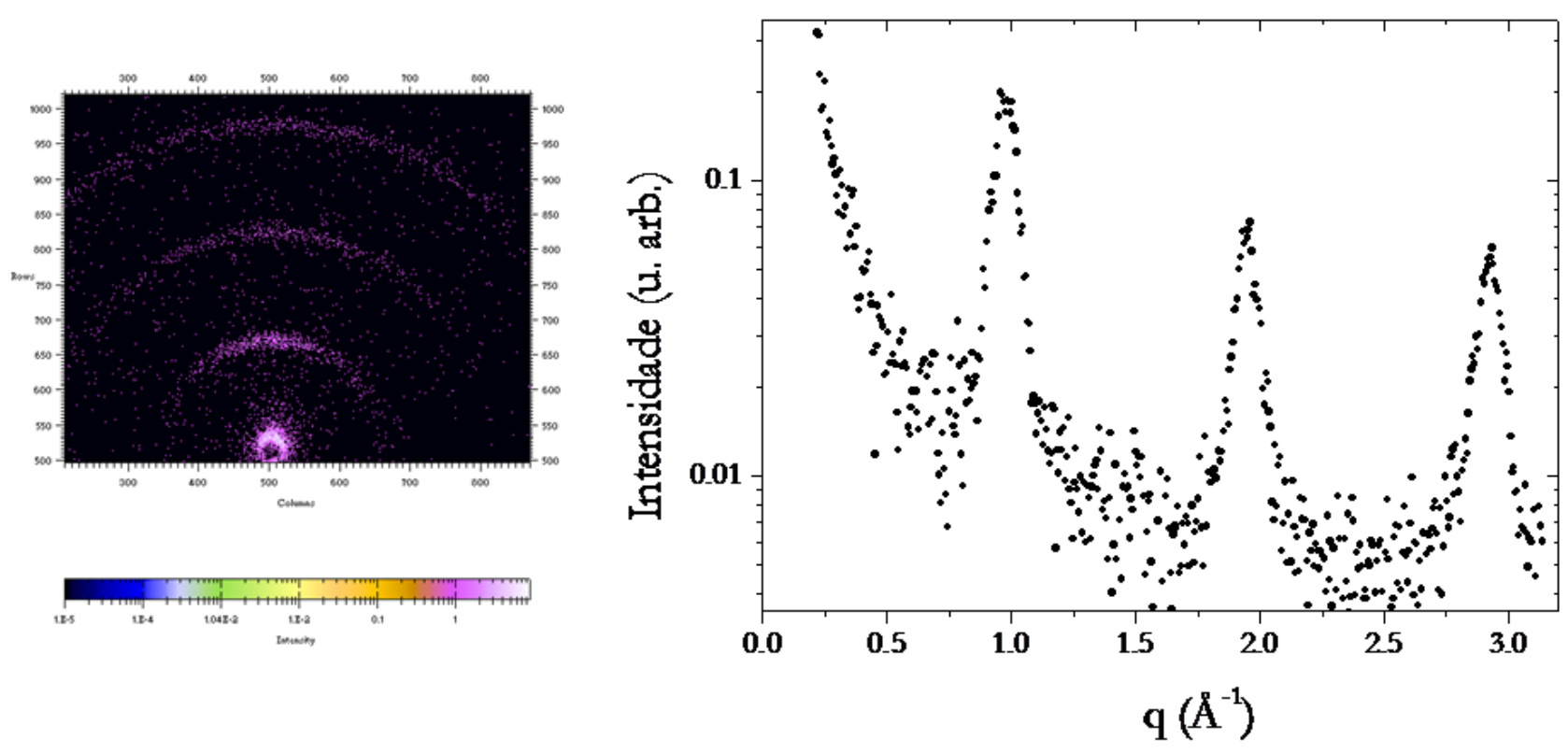

Figura 3. I 2.: (a) Difratograma obtido para o BeAg e (b) Intensidade em função do vetor de onda $\mathrm{q}$, nos experimentos de GISAXS.

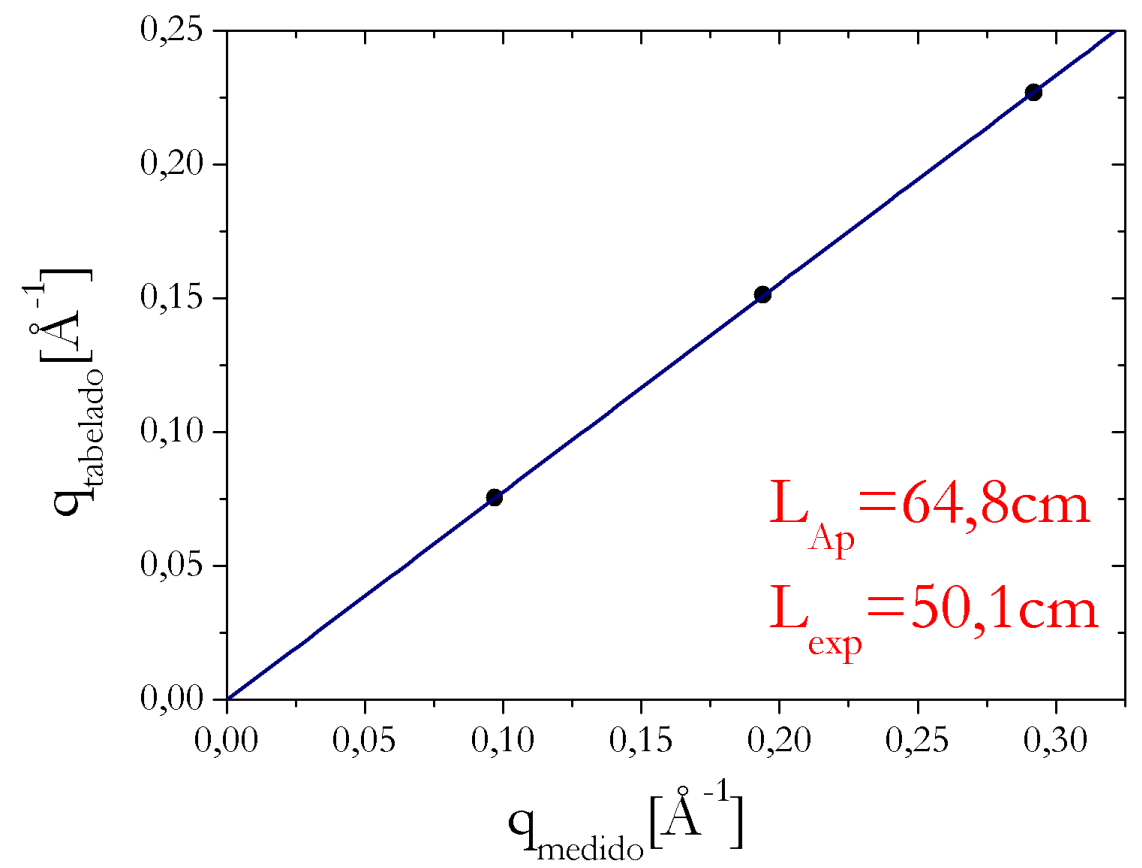

Figura 3.ı 3.: (a) Calibração da distância do detector-amostra para os experimentos de GISAXS.

Barbara Bianca Gerbelli - Propriedades estruturais e elásticas de fases lamelares: O efeito da composição da membrana. 

Nesse capítulo será apresentadp os resultados experimentais obtidos no estudo estrutural da fase lamelar variando a composição da membrana. Em primeiro lugar serão investigadas as propriedades estruturais de membranas compostas apenas por lecitina. Em seguida a investigação do efeito da incorporação do co-surfactante comparando as alterações nos parâmetros estruturais e no parâmetro de Caillé. Também serão apresentados os resultados experimentais obtidos no estudo termodinâmico para duas composições de membrana, que levam à determinação do módulo de compressibilidade. A combinação desses resultados com o parâmetro de Caillé permite a determinação da constante de rigidez da membrana.

\subsection{Estudo Estrutural}

\subsubsection{Membranas de lecitina}

\section{Apresentação dos resultados}

O padrão de espalhamento característicos de fases lamelares registrados em um detector bidimensional (Figura 4. I) consiste de anéis concêntricos e igualmente espaçados. Algumas imagens típicas são mostradas na figura 4.I para diferentes diluições. Em alguns difratogramas a intensidade espalhada não se dá de maneira uniforme, apresentando reforço em alguma direção (Figura 4.I (e)). Esse efeito pode ser explicado pela existência de pequenos domínios orientados que são gerados no processo de inserção das amostras nos capilares de vidro por centrifugação.

Barbara Bianca Gerbelli - Propriedades estruturais e elásticas de fases lamelares: O efeito da composição da membrana. 


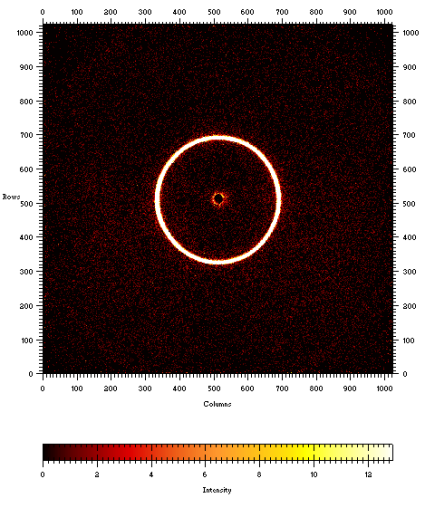

(a) $\phi_{\text {lip }}=0,87$

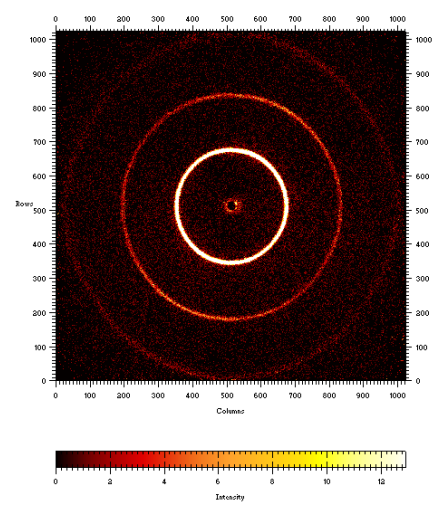

(d) $\phi_{\text {lip }}=0,73$

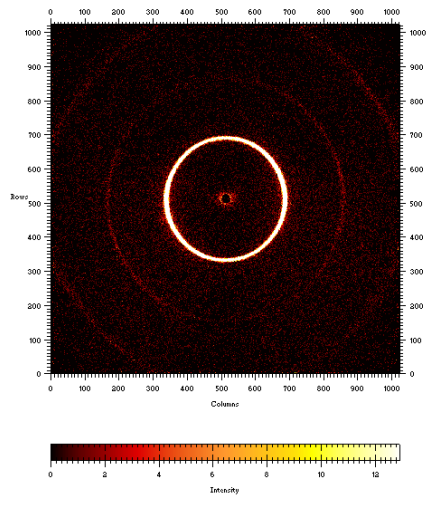

(b) $\phi_{\text {lip }}=0,86$

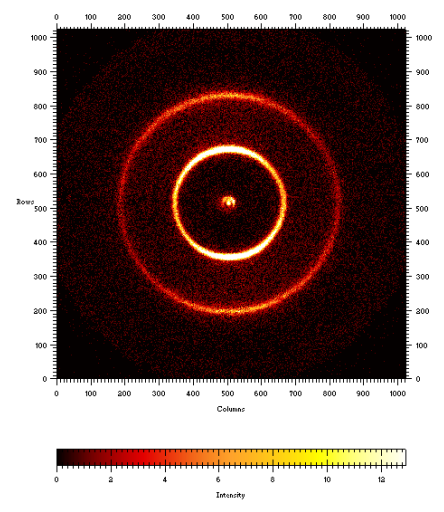

(e) $\phi_{\text {lip }}=0,60$

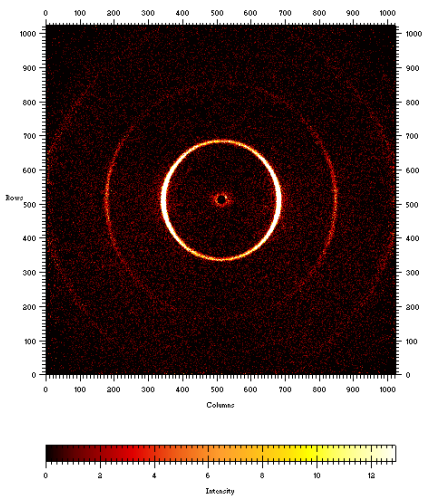

(c) $\phi_{\text {lip }}=0,78$

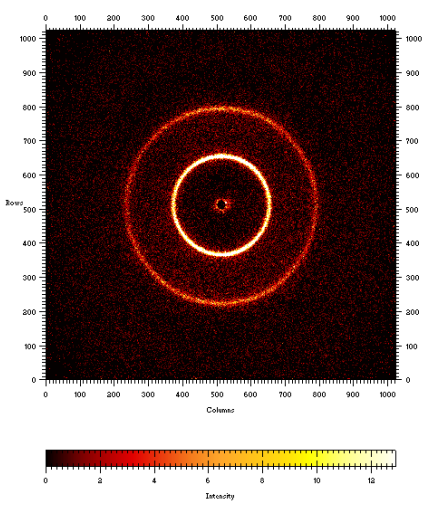

(f) $\phi_{\text {lip }}=0,58$

Figura 4. I.: Difratogramas de fases lamelares compostas de lecitina em diferentes díluções.

É realizada a integração dos difratogramas onde os dados reduzidos são curvas unidimensionais que representam a intensidade espalhada $(\mathrm{I}(\mathrm{q})$ ) em função do vetor de onda $q$, apresentadas na figura 4.2. Para todo o domínio estudado observa-se uma fase lamelar. À medida que o sistema é hidratado observa-se claramente um deslocamento dos picos de Bragg para valores de $q$ pequenos. Utilizando a lei de Bragg pode-se obter a periodicidade lamelar $\boldsymbol{q}_{1}=\frac{2 \pi}{D}$, onde $\boldsymbol{q}_{1}$ é o vetor de onda do pico de primeira ordem.

Considerando que a espessura da bicamada não é alterada com a hidratação, com um modelo geométrico simples (descrito no apêndice C) pode-se obter uma relação entre a fração volumétrica de lipídio $\left(\phi_{\text {lip }}\right)$ na solução aquosa e a periodicidade lamelar (equação C.3).

$$
D=\frac{\delta_{M}}{\phi_{l i p}}
$$

Barbara Bianca Gerbelli - Propriedades estruturais e elásticas de fases lamelares: O efeito da composição da membrana. 


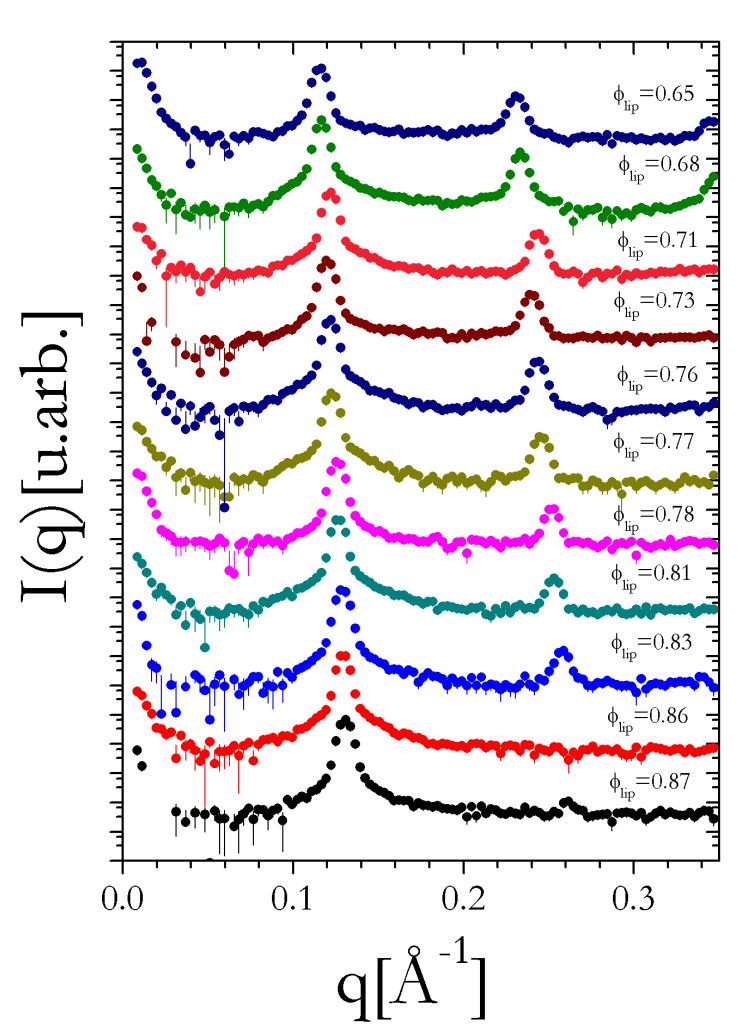

(a)

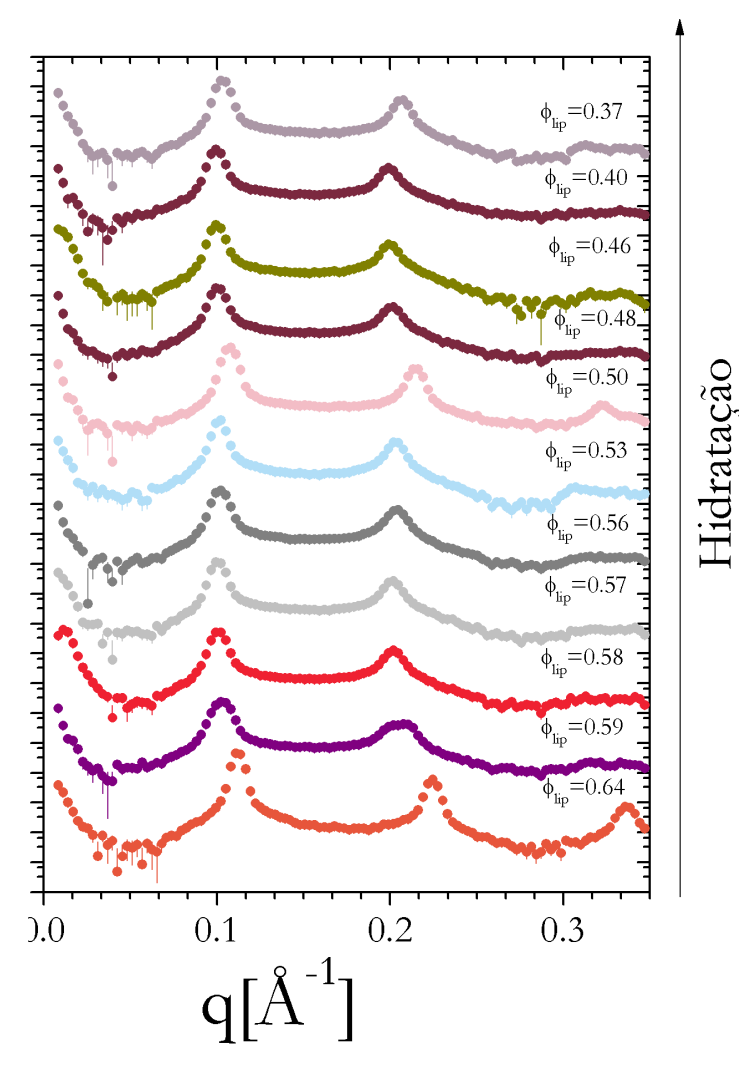

(b)

Figura 4.2.: Curvas de I(q) vs. q para fases lamelares compostas de lecitina em diferentes diluições.

De acordo com a equação C.3 podemos construir um gráfico de D vs. $1 / \phi_{\text {lip }}$ onde se espera uma reta cujo coeficiente angular é a espessura da membrana. Na Figura 4.3 são observadas duas regiões distintas; na primeira região os pontos seguem um comportamento linear, porém não seguem a lei de diluição usual (eq. C. 5). Essa região corresponde à periodicidade lamelar variando de $48 \AA$ á $62 \AA$, porém a reta que ajusta os pontos não passa pela origem. Isso mostra que o modelo de membranas rígidas não é adequado para descrever o comportamento observado. Na segunda região, a partir de $\phi_{\text {lip }}=\mathbf{0 , 6 5}$, a periodicidade lamelar se mantém constante $\left(D_{\max }\right)$ em torno de $\mathbf{6 2}, 8 \AA$, indicando o limite de hidratação da membrana. Isto é, pode-se aumentar a quantidade de água e mesmo assim a periodicidade lamelar se mantém constante. Acima dessa fração volumétrica de lipídio a fase lamelar com periodicidade máxima coexiste com excesso de água.

Utilizou-se o método de análise de deconvolução gaussiana descrito no Capítulo 2 para

Barbara Bianca Gerbelli - Propriedades estruturais e elásticas de fases lamelares: O efeito da composição da membrana. 


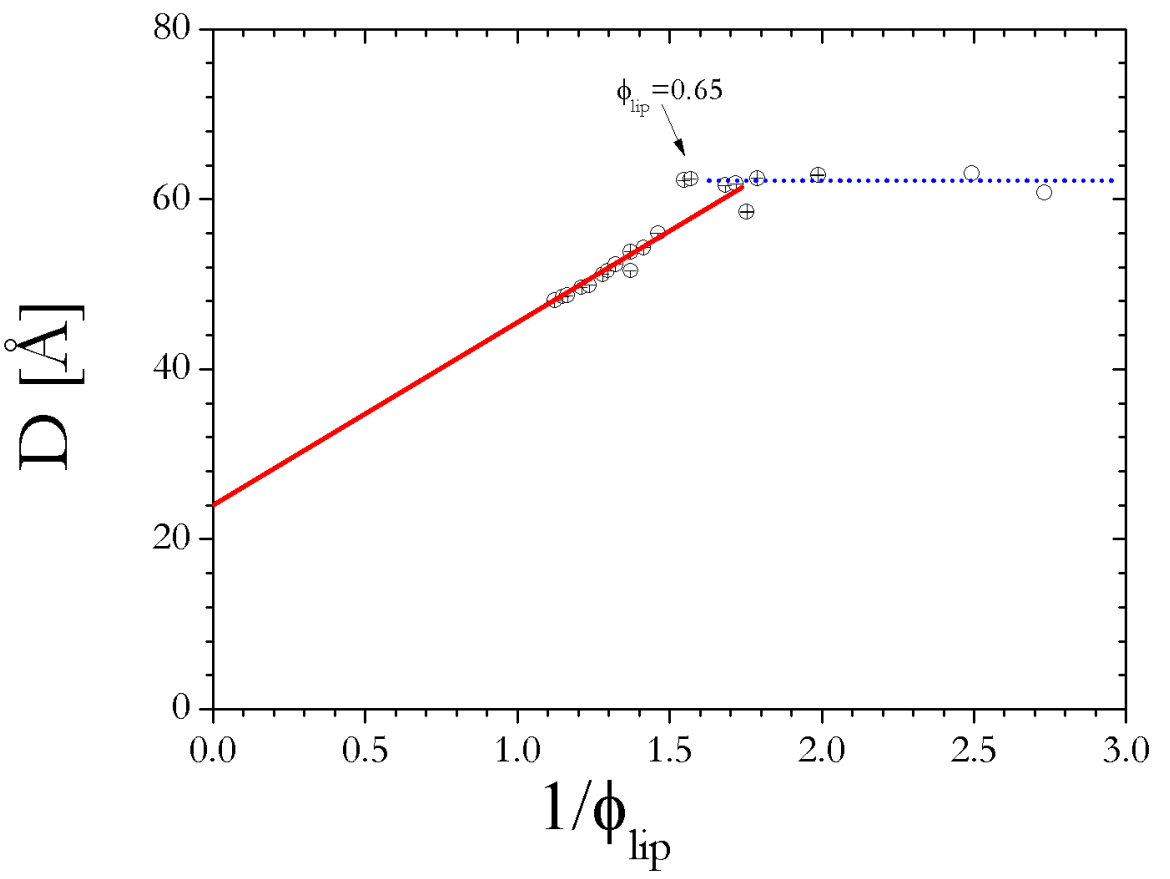

Figura 4.3.: Periodicidade lamelar $v$ s. $1 / \phi_{\text {lip }}$.

o ajuste dos dados experimentais de espalhamento. Obtém-se, então, para cada curva o fator de forma $(\mathrm{P}[\mathrm{q}])$ e o fator de estrutura $(\mathrm{S}[\mathrm{q}])$ que melhor ajustam a intensidade de espalhamento. A comparação das curvas experimentais e o ajuste obtido é apresentada na figura 4.4 como as respectivas funções $P[q]$ e S[q].

Observa-se na figura 4.4, que para altas concentrações de lipídio $\left(\phi_{\text {lip }} \approx 0,86-\right.$ $0,87)$ o pico de Bragg de segunda ordem está ausente ou pouco visível reaparecendo para concentração de $\phi_{l i p}=0,83$. A ausência do pico de segunda ordem é incompatível com o aumento da quantidade de lipídio, pois seria esperado o aumento do comprimento de correlação da ordem esmética. Isso pode ser explicado pelo fato de que, para essas concentrações o mínimo local do fator de forma ocorre para um valor de $q$ próximo do pico de segunda ordem do fator de estrutura. O conjunto dos resultados obtidos mostra que o fator de forma não se mantém constante com a hidratação da membrana, evidenciando alterações na estrutura da bicamada.

O ajuste dos dados experimentais fornece também o valor da periodicidade lamelar compatível com as calculadas pela posição do pico de Bragg como descrito acima, porém a determinação da espessura da bicamada segue o procedimento descrito na seção 3.3.3. O comportamento desses parâmetros em função da fração volumétrica de lipídio é apresentado na figura 4.5. Nessa figura observa-se que para frações volumétricas inferiores a

Barbara Bianca Gerbelli - Propriedades estruturais e elásticas de fases lamelares: O efeito da composição da membrana. 
0,65 , a periodicidade lamelar se mantém praticamente constante.

$\mathrm{Na}$ figura 4.6 são apresentados os perfis de contraste eletrônico para duas frações volumétrica de lipídios deslocados de uma periodicidade lamelar. Para a amostra com fração volumétrica de lipídio igual a 0,87 observa-se a superposição correspondente a região das cabeças polares enquanto para $\phi_{\text {lip }}=0,73$ o contraste entre a região polar e a camada aquosa é bem nítido. A superposição das cabeças polares é acompanhada do aumento da área por cabeça polar $\left(\sum\right)$ sendo acompanhada de uma diminuição da espessura da bicamada C.6.

A partir de $\phi_{\text {lip }} \gtrsim \mathbf{0 , 6 5}$ a desidratação da bicamada resulta não apenas na redução da periodicidade lamelar, mas também produz alterações na espessura da bicamada, que passa de $35 \AA$ para 41 A. Esse efeito mostra que a desidratação da fase lamelar não consiste apenas na remoção de moléculas de água da camada aquosa entre as bicamadas, e que, a desidratação tem efeitos também sobre a estrutura da membrana. Uma possível interpretação para o arranjo das moléculas de lipídio na interface é representada na figura 4.7.

Para obter informações referentes à organização das caudas hidrocarbonadas utilizou-se a técnica de WAXS. Para esses experimentos foram escolhidas três composições distintas; uma no regime muito concentrado, uma no limite de hidratação e uma intermediária. Os experimentos foram realizados á várias temperaturas $5,10,22$ e $35,5^{\circ} \mathrm{C}$. Os dados obtidos são apresentados na figura 4.8 onde observa-se um pico difuso em torno de $1,5 \AA$, para todas as composições e temperaturas estudas. Este pico corresponde à distância média entre as caudas carbônicas, igual á 4, 4 Å. A largura do pico não é alterada evidenciando o caráter líquido na organização das cadeias carbônicas. Sendo assim, conclui-se que em todo o domínio de diluição estudado, e no intervalo de temperatura considerado, todas as amostras apresentam se na fase fluída $\left(\boldsymbol{L}_{\boldsymbol{\alpha}}\right)$. Além do pico difuso, em algumas curvas observou-se um segundo pico difuso com menor intensidade indicado pela seta que se refere ao espalhamento das moléculas de água.

Barbara Bianca Gerbelli - Propriedades estruturais e elásticas de fases lamelares: O efeito da composição da membrana. 


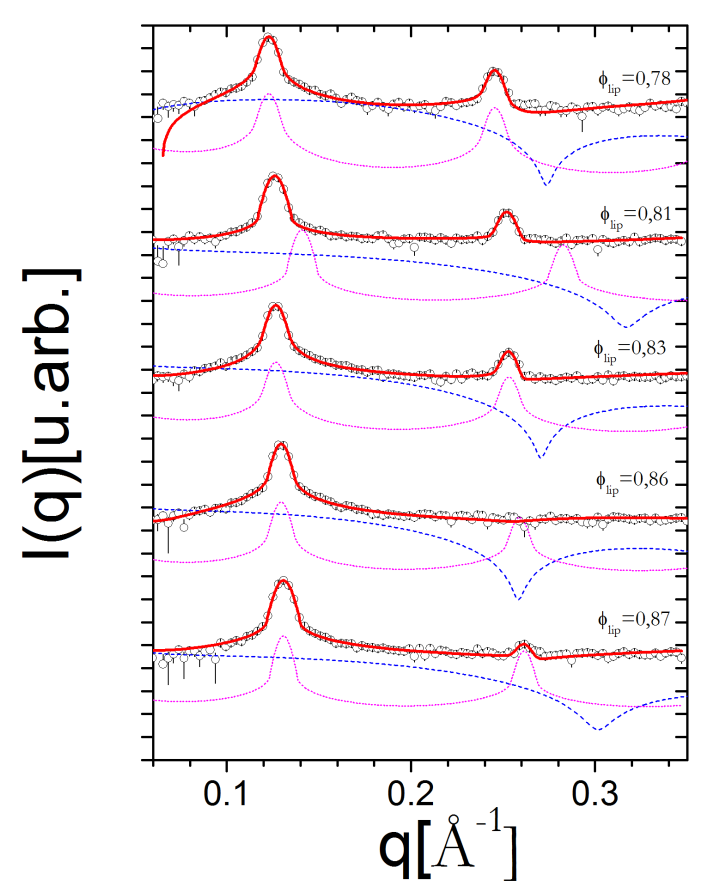

(a)

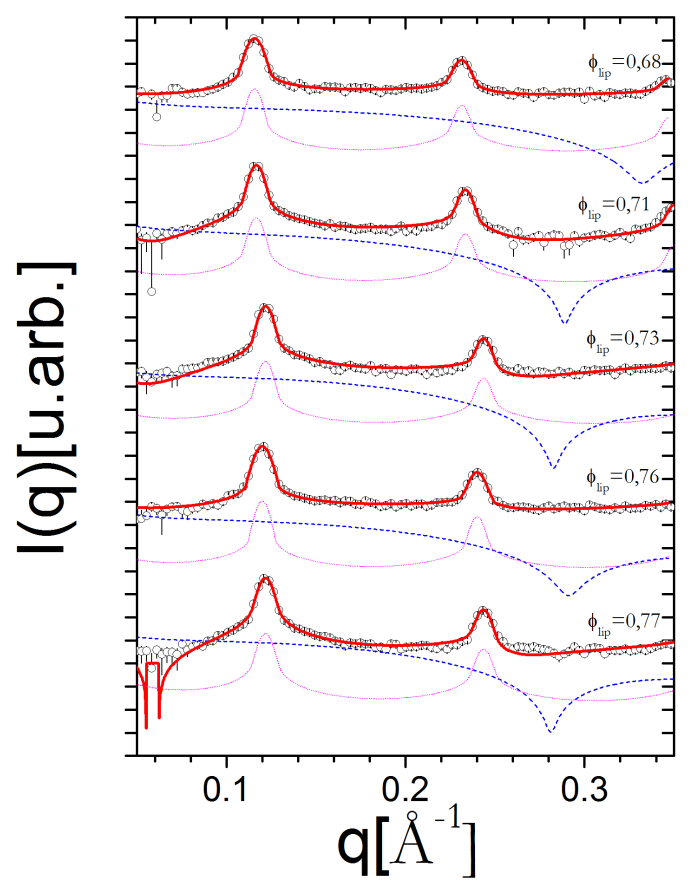

(c)

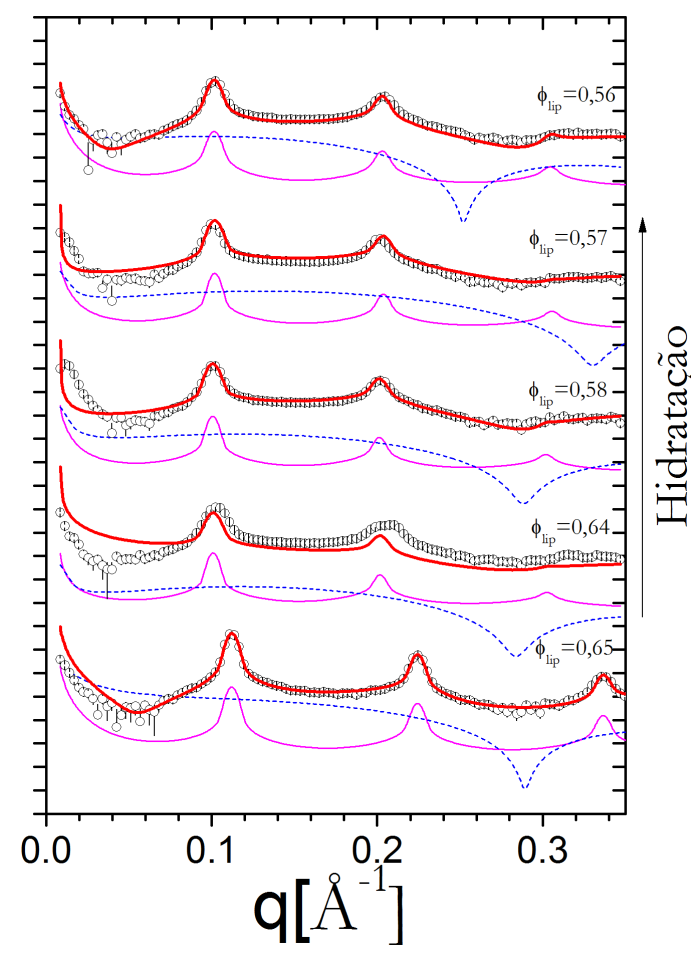

(b)

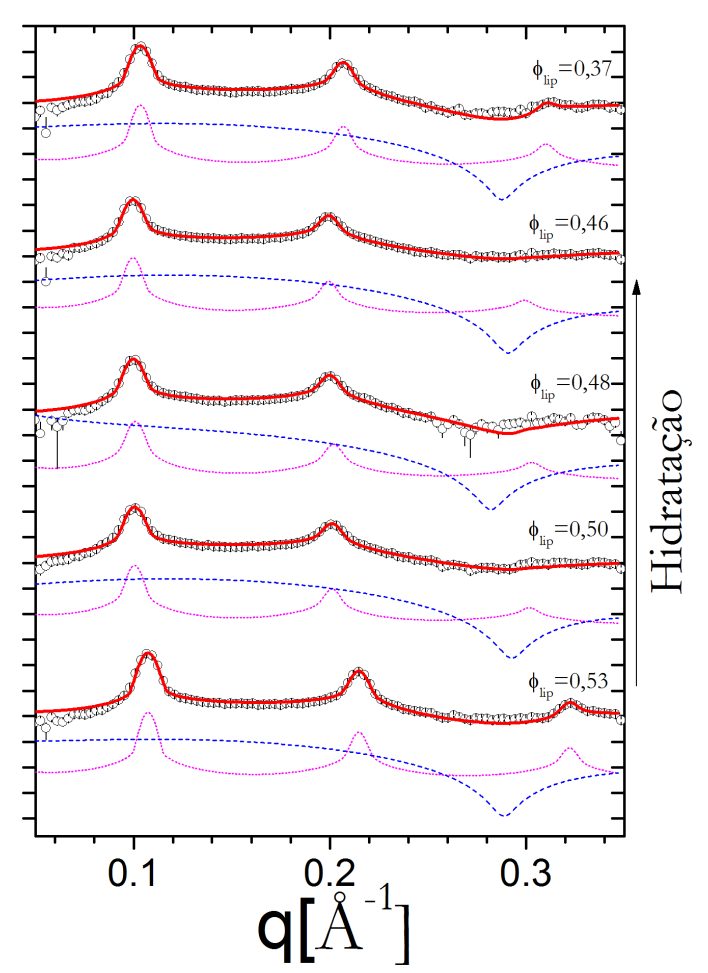

(d)

Figura 4.4.: Dados ajustados para diversas frações volumétricas de lecitina. Os pontos representam os dados experimentais, a linha cheia representa o ajuste dos dados, a linha pontilhada representa o fator de forma e a linha tracejada o fator de estrutura.

Barbara Bianca Gerbelli - Propriedades estruturais e elásticas de fases lamelares: O efeito da composição da membrana. 


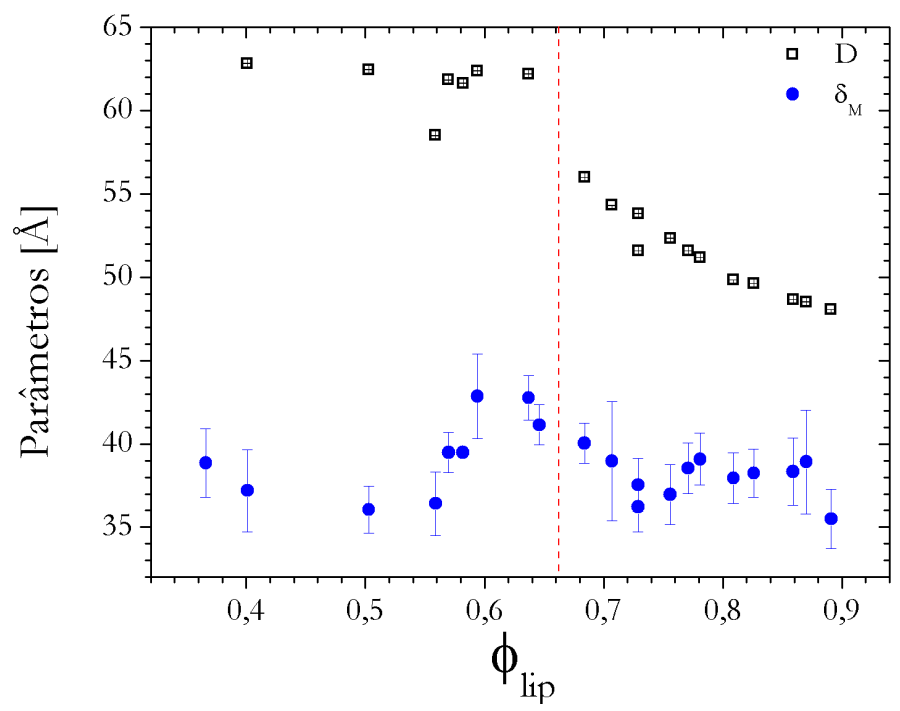

Figura 4.5.: Parâmetros estruturais (D e $\delta_{M}$ ) em função de $\phi_{l i p}$

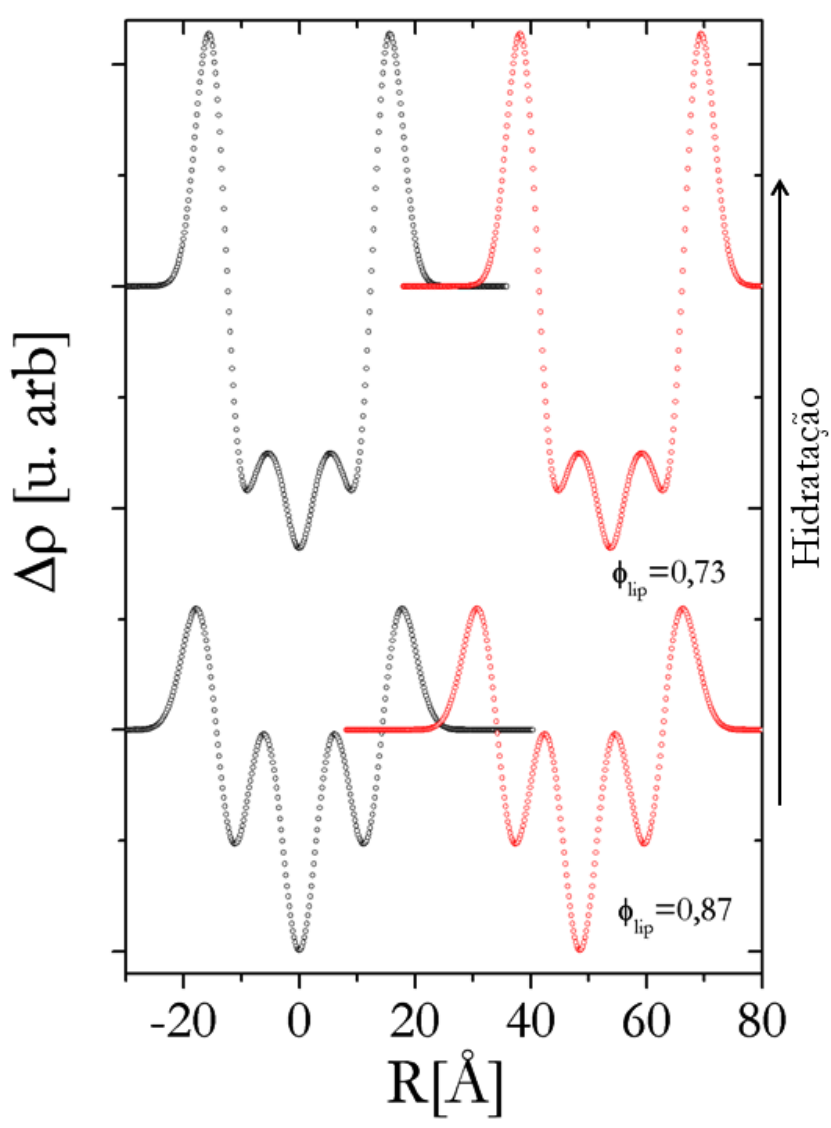

Figura 4.6.: Contraste de densidade eletrônica para duas frações volumétricas de lipídio.

Barbara Bianca Gerbelli - Propriedades estruturais e elásticas de fases lamelares: O efeito da composição da membrana. 

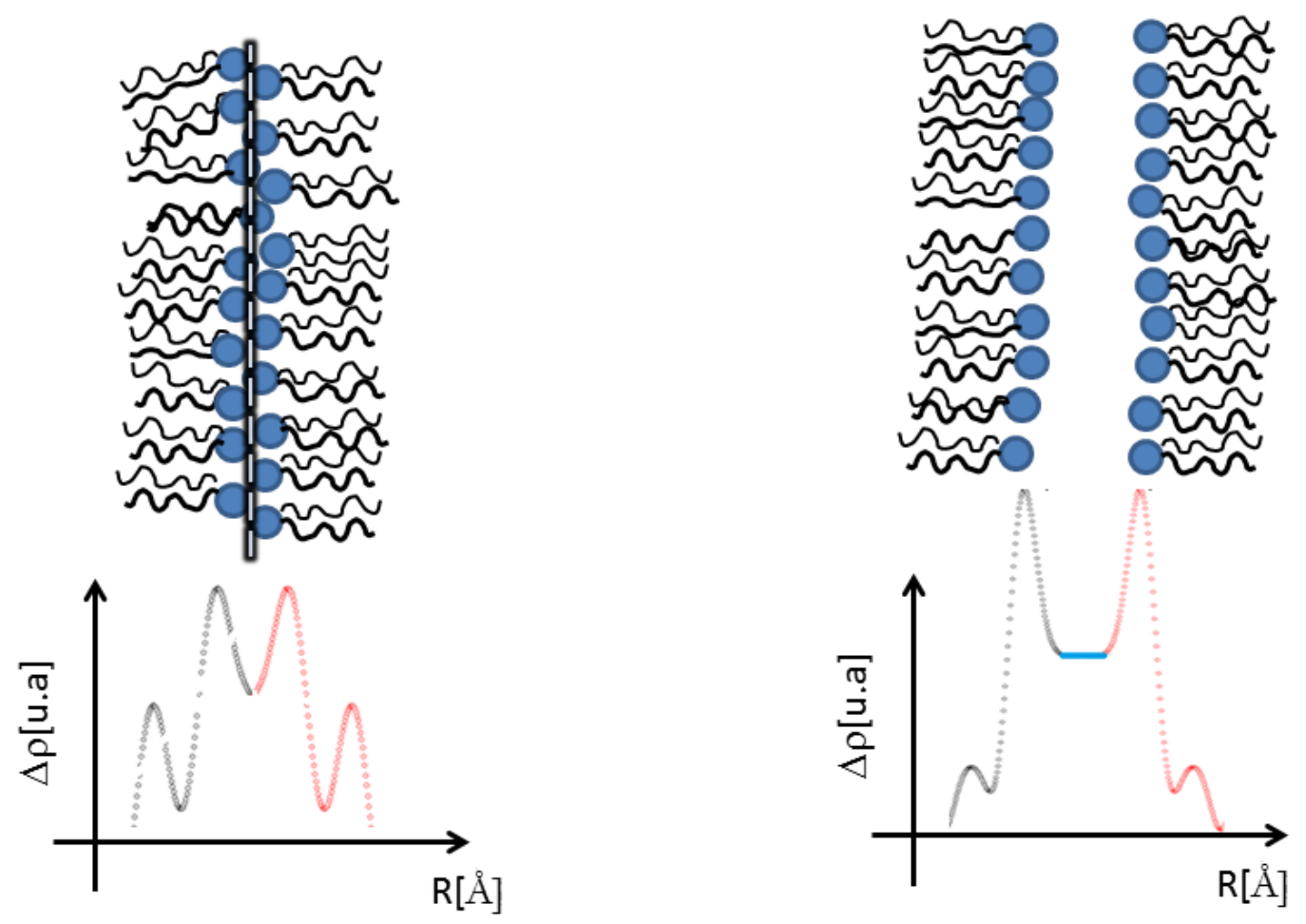

Figura 4.7.: Representação esquemática da interface cabeças polares-água de acordo com o perfil de densidade eletrônica obtido do ajuste dos dados experimentais de espalhamento de raios X; (a) No regime concentrado com superposição da região polar da bicamada e, (b) no regime diluído, com as regiões polares da bicamada separadas por uma camada aquosa.

Barbara Bianca Gerbelli - Propriedades estruturais e elásticas de fases lamelares: O efeito da composição da membrana. 


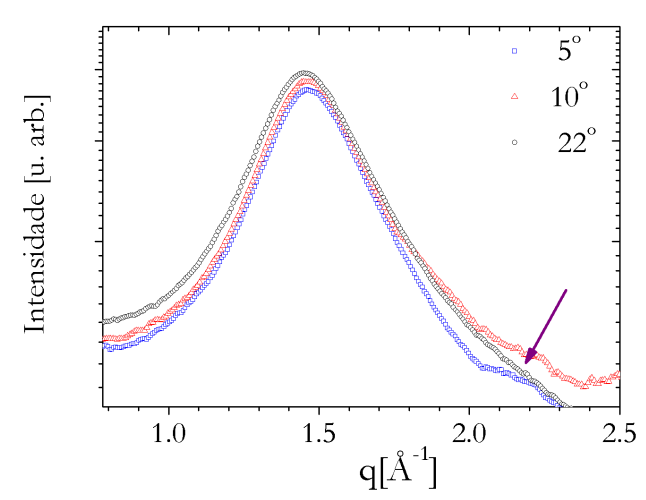

(a) $\phi_{\text {lip }}=0,82$

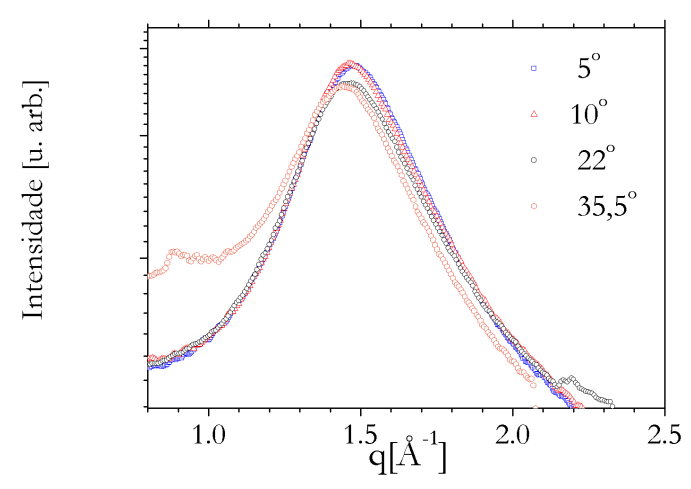

(b) $\phi_{\text {lip }}=0,77$

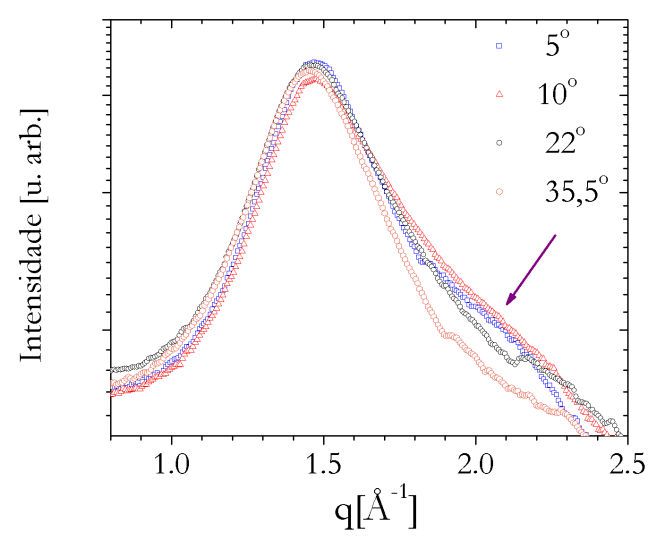

(c) $\phi_{\text {lip }}=0,68$

Figura 4.8.: Dados de WAXS para três frações de lipídio diferentes, variando-se a temperatura no intervalo de 5 a $35,5^{\circ} \mathrm{C}$

Barbara Bianca Gerbelli - Propriedades estruturais e elásticas de fases lamelares: O efeito da composição da membrana. 
O outro parâmetro que é obtido do ajuste das curvas experimentais é o parâmetro de Caillé $(\boldsymbol{\eta}$,$) que está relacionado às propriedades elásticas da membrana. O comporta-$ mento desse parâmetro $(\boldsymbol{\eta})$ é representado na figura 4.9 onde pode-se observar três regimes distintos. O primeiro regime, onde $\boldsymbol{\eta}$ é praticamente constante, corresponde a região da coexistência da fase lamelar com hidratação máxima e excesso de água. $\mathrm{Na}$ segunda região observa-se um decrescimento exponencial do parâmetro de Caillé até aproximadamente $\phi_{\text {lip }}=0,8$. No terceiro regime, correspondente a região de forte confinamento das bicamadas, $\boldsymbol{\eta}$ apresenta novamente um valor constante e muito pequeno, próximo de $\mathbf{1 0}^{-\mathbf{2}}$. Nesse regime a distância típica de separação entre as bicamadas é menor que $8 \AA$.

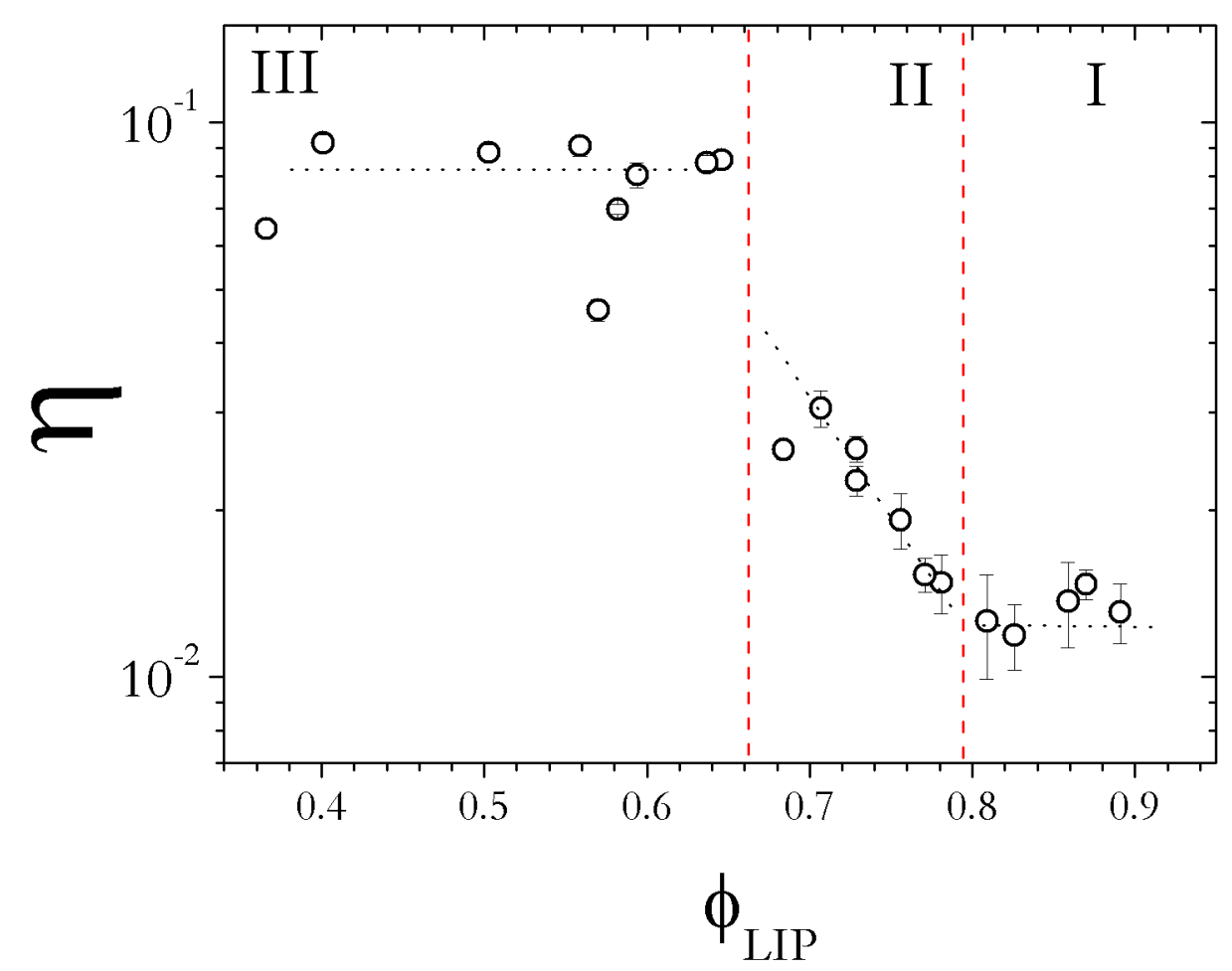

Figura 4.9.: Parâmetro de Caillé em função de $\phi_{l i p}$.

A partir do parâmetro de Caillé pode-se calcular a amplitude quadrática das flutuações da espessura da camada aquosa $\left(\sigma^{2}\right)$ de acordo com a equação 2. I I. A figura 4. Io ilustra o comportamento de $\boldsymbol{\sigma}$ em função da periodicidade lamelar, que é comparável com resultados existentes na literatura para a lecitina de ovo, no intervalo de periodicidade lamelar compreendido entre 50 e $60 \AA$ [ [22]. Em ambos os gráficos observa-se o crescimento aproximadamente linear de $\sigma$ com a hidratação das membranas, mas a taxa de crescimento de $\sigma$ não é constante. A amplitude de flutuação da distância de separação entre as membra-

Barbara Bianca Gerbelli - Propriedades estruturais e elásticas de fases lamelares: O efeito da composição da membrana. 
nas no limite de diluição atinge o valor máximo, da ordem de 6 A o que é compatível com dados disponíveis na literatura.

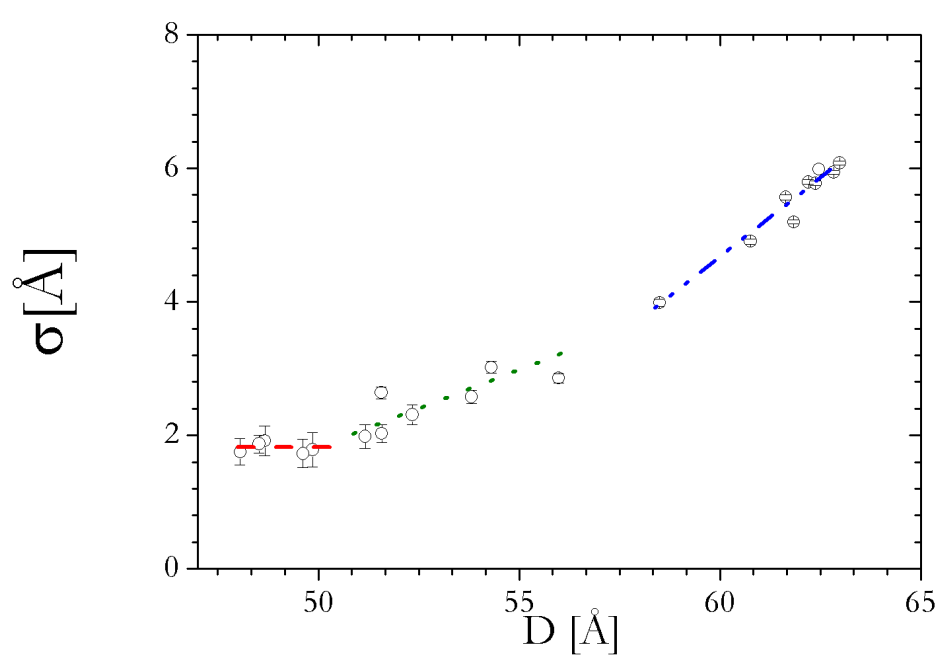

(a) Lecitina de soja

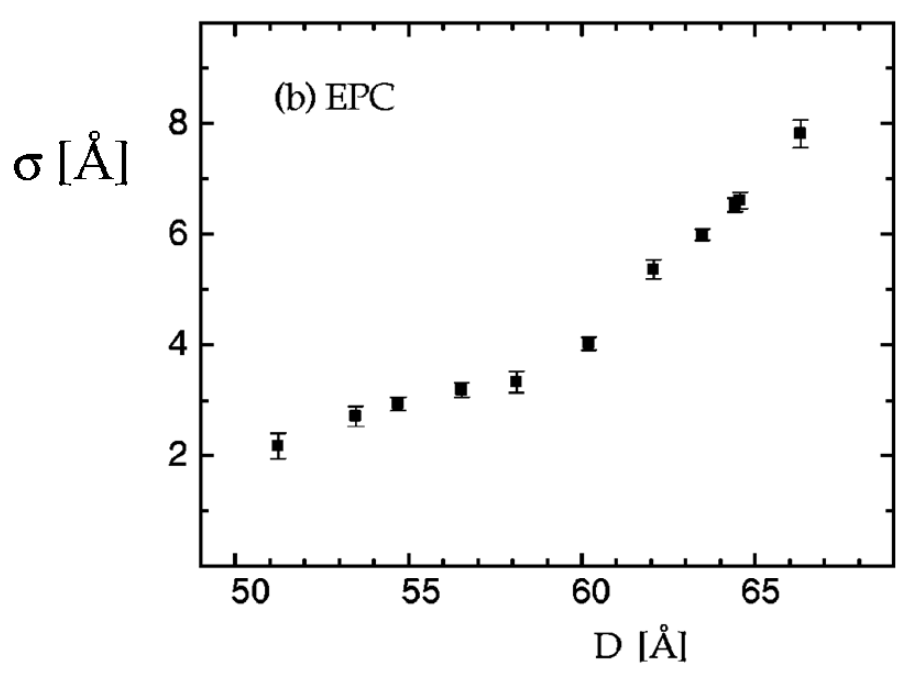

(b) Lecitina de ovo

Figura 4. Io.: Amplitude das flutuações da espessura da camada aquosa em função da periodicidade lamelar. Lecitina de (a) soja (b) ovo. Na figura (a) as linhas pontilhadas são apenas guia para os olhos. A figura (b) foi adaptada da ref. [22]

Barbara Bianca Gerbelli - Propriedades estruturais e elásticas de fases lamelares: O efeito da composição da membrana. 


\section{Discussão dos resultados}

Nesta seção foram apresentados os resultados obtidos na investigação das propriedades estruturais da fase lamelar de lecitina de soja. Foi observado que, em todo domínio de concentração e temperatura explorados, as membranas se mantém na fase fluida.

Existem na literatura vários estudos de fases lamelares de lecitina, porém neste trabalho a aplicação do método de deconvolução gaussiana para o ajuste dos dados experimentais de espalhamento de raios $\mathrm{X}$ revelou novos aspectos sobre a estrutura e propriedades elásticas desse sistema. Neste trabalho foi explorado um regime fortemente concentrado, trazendo à luz novos aspectos sobre a estrutura da fase lamelar que não estão relatados na literatura.

Uma vantagem do método utilizado para obter a informação da variação da espessura da bicamada, é que esta é extraída do perfil de densidade eletrônico, não dependendo, portanto, de modelos que se baseiam na conformação das caudas carbônicas ou grupos polares. Essa abordagem permite uma descrição mais detalhada da estrutura da fase lamelar. Os resultados aqui apresentados mostram que a desidratação da fase lamelar não consiste apenas na remoção de água do espaço entre as bicamadas, mas afeta também a estrutura da membrana com consequência em suas propriedades elásticas.

Foi observada uma variação da espessura da bicamada em torno de 5 Å compatível com previsões de Luzzati utilizando argumentos geométricos [29]. Na literatura, a variação da espessura relatada para a bicamada é da ordem de $2 \AA$ [43], porém o modelo utilizado para descrever a modulação da densidade eletrônica assume previamente uma configuração das moléculas de lipídios [45] [53] [54].

O parâmetro de Caillé é de importância fundamental para revelar mudanças no regime de interações entre as membranas. Os dados experimentais no regime confinado cobrem distâncias de separação entre as membranas de 5 á 8 Å e nesse domínio, embora a quantidade de água aumente, o parâmetro de Caillé se mantém constante, indicando que as moléculas de água incorporadas provavelmente passam a se ligar fortemente aos grupos polares. A dependência exponencial do parâmetro de Caillé não é descrita por modelos existentes na literatura. No intervalo onde o parâmetro de Caillé varia, o mecanismo de ondulação, previsto por Helfrich, provavelmente compete com as forças de hidratação (repulsiva) e van der Waals (atrativa).

É possível que existam outros modos de flutuação, como por exemplo peristáltico, que sejam relevantes, além de interações dipolares entre as moléculas de água com os grupos polares das moléculas anfifílicas [34].

Barbara Bianca Gerbelli - Propriedades estruturais e elásticas de fases lamelares: O efeito da composição da membrana. 


\subsubsection{Variação na composição da membrana}

Nesta seção serão apresentados os resultados obtidos no estudo estrutural da fase lamelar variando a composição da membrana com a incorporação do co-surfactante Simulsol. Para cada composição de membrana foi explorada a linha de diluição. A análise dos dados experimentais de raios $\mathrm{X}$ foi realizada seguindo a mesma metodologia descrita na seção 3.3.3. Para algumas composições da membrana, ao fazer a varredura na linha de diluição, foi identificado um domínio bifásico, caracterizado pela presença de um pico de Bragg extra na região de baixo ângulo. Em alguns casos foi possível identificar apenas um pequeno ombro superposto ao pico de primeira ordem da fase lamelar predominante, como ilustrado na figura 4. I I.

A incorporação de até ı \% em massa de Simulsol à membrana não resulta em domínios bifásicos, e para composições de membrana contendo entre $10 \%$ e $50 \%$ em massa de Simulsol o domínio bifásico ocorre para frações volumétricas de lipídio em torno de $\mathbf{0 , 7}$. A partir daí, a incorporação de mais Simulsol desloca o domínio bifásico para fração volumétrica de lipídio em torno $\mathbf{0 , 8}$. Esse domínio bifásico corresponde à coexistência de duas fases lamelares; uma mais diluída e uma mais concentrada. O diagrama de fase à temperatura constante é apresentado na figura 4. 12 onde os pontos correspondem à observações experimentais de coexistência de fase. A identificação dos limites precisos desse domínio necessitam, no entanto, de uma investigação mais detalhada.

O comportamento da periodicidade lamelar em função de $1 / \phi_{\text {lip }}$ é mostrado na figura 4. I3, para algumas composições de membrana. Como no caso da lecitina pura, duas regiões com comportamentos diferentes são observadas. A primeira segue um crescimento linear semelhante ao comportamento observado para membranas compostas de lecitina. Com a inserção de Simulsol à membrana a inclinação da reta aumenta, indicando um aumento na espessura da bicamada. Na segunda região que corresponde ao limite de diluição, observa-se que para pequenas quantidades de Simulsol é possível identificar uma periodicidade máxima que, no entanto, aumenta com o aumento da quantidade de Simulsol. Aumentando-se ainda mais a quantidade de Simulsol na membrana, esse limite não é claramente identificado. Para uma mesma fração volumétrica de lipídio, diferentes periodicidades lamelares podem ser obtidas variando-se a quantidade de Simulsol.

Barbara Bianca Gerbelli - Propriedades estruturais e elásticas de fases lamelares: O efeito da composição da membrana. 


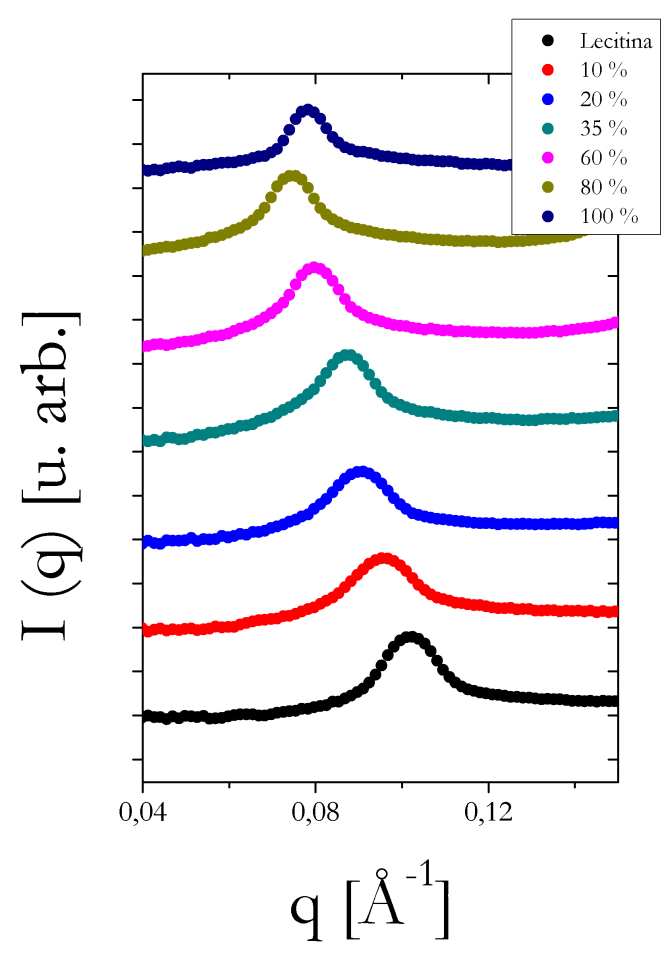

(a) $\phi=0,6$

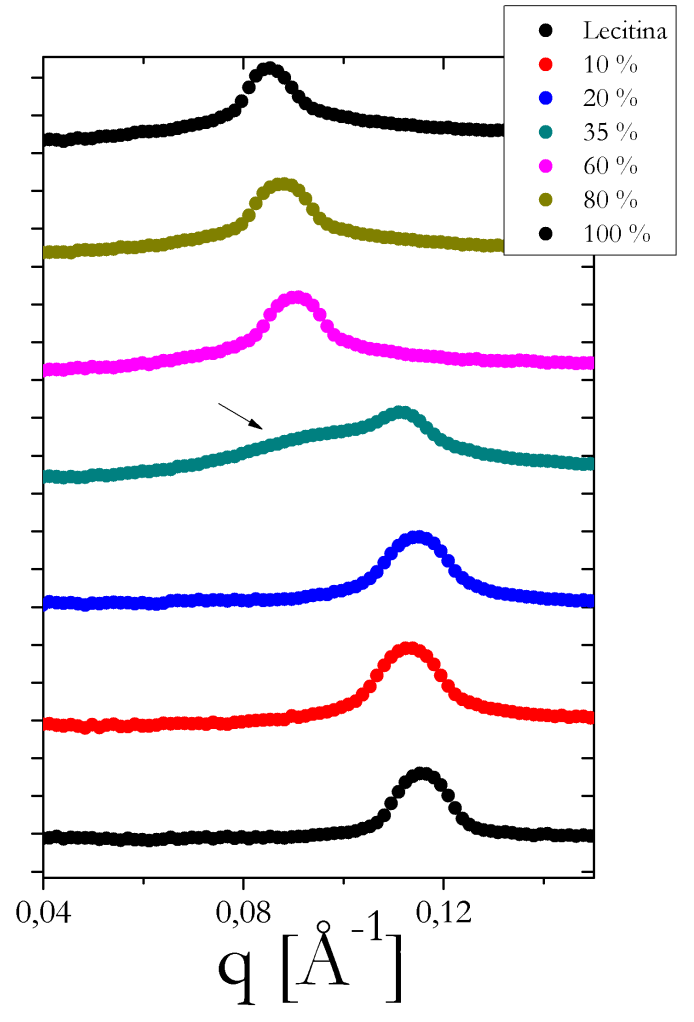

(b) $\phi=0,7$

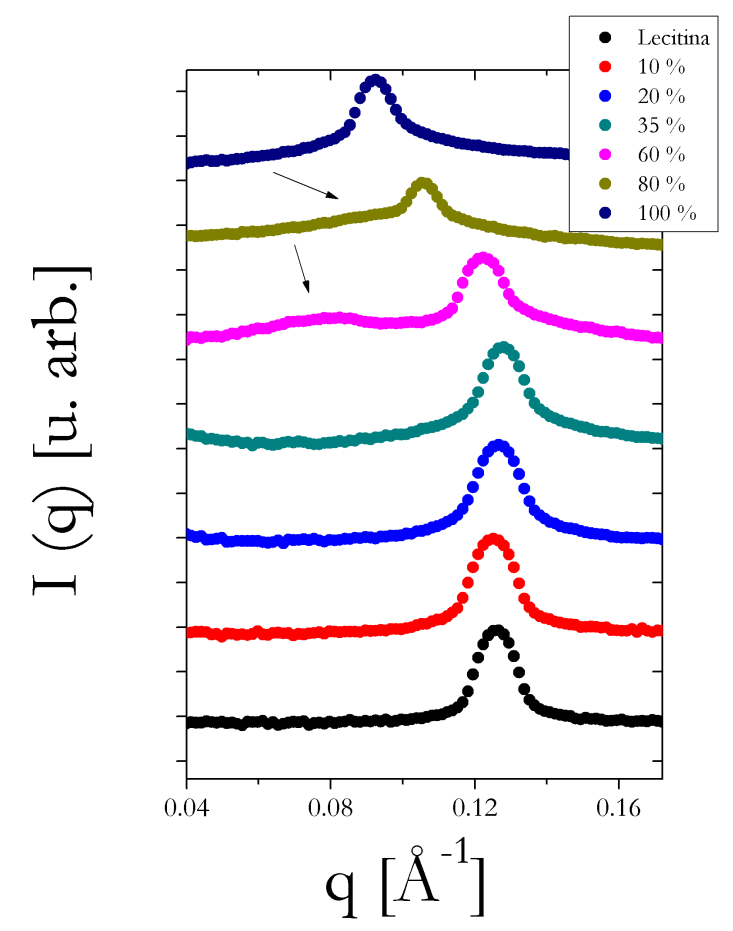

(c) $\phi=0,8$

Figura 4. I I.: Curvas de espalhamento I(q) obtidos para fases lamelares com diferentes composições de membrana (\% em massa de Simulsol).

Barbara Bianca Gerbelli - Propriedades estruturais e elásticas de fases lamelares: O efeito da composição da membrana. 


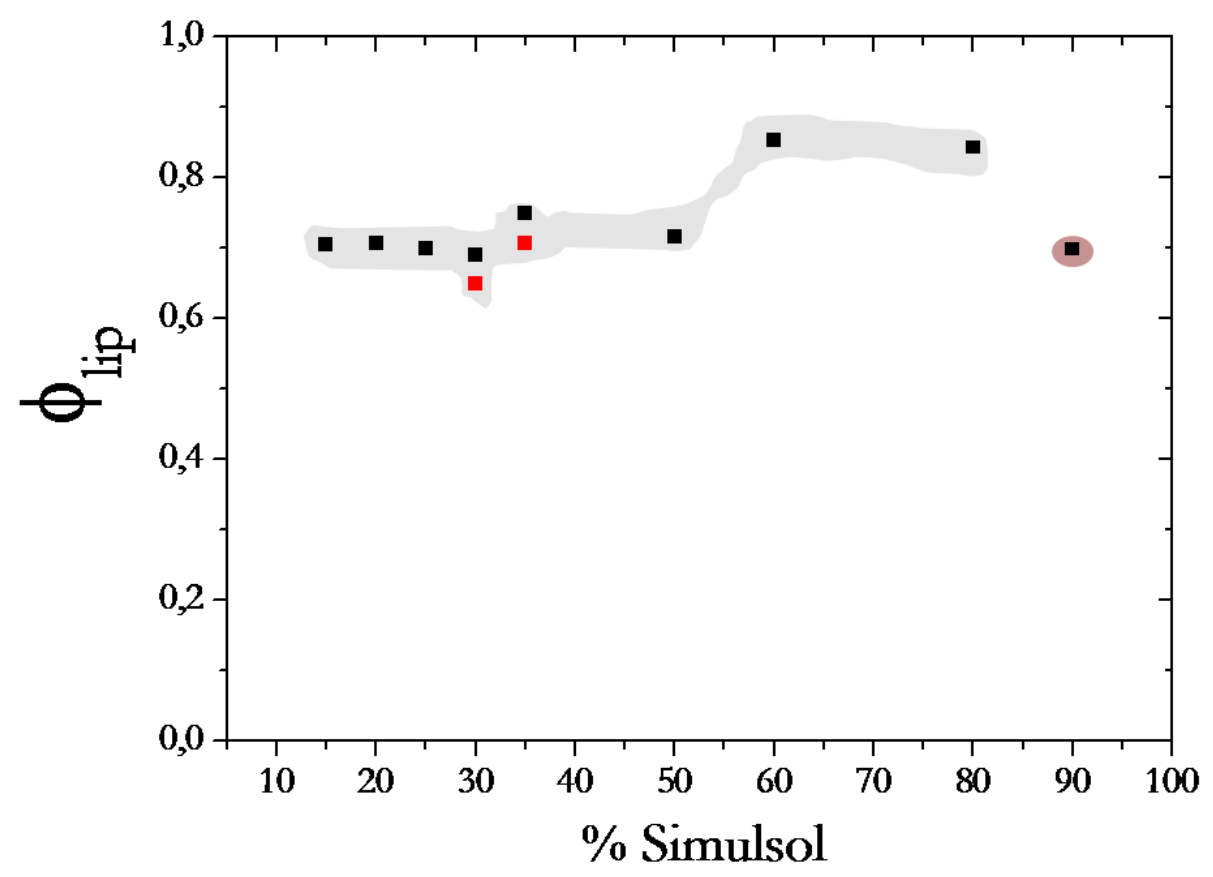

Figura 4. I 2.: Diagrama da fase lamelar contendo Simulsol, com a identificação de um domínio de coexistência de duas fases lamelares, representado pelos pontos experimentais (\% Simulsol em massa).

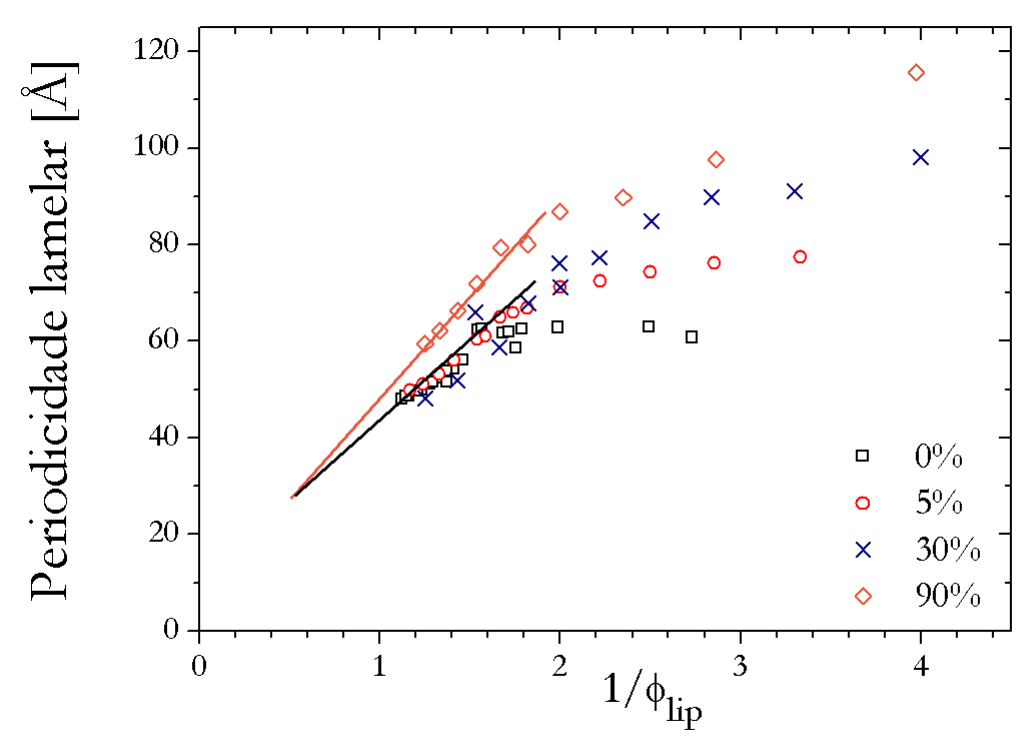

Figura 4. I 3.: Periodicidade lamelar vs. $1 / \phi_{\text {lip }}$ variando a quantidade de Simulsol incorporado à membrana. As linhas são apenas um guia para os olhos.

Barbara Bianca Gerbelli - Propriedades estruturais e elásticas de fases lamelares: O efeito da composição da membrana. 
$\mathrm{Na}$ figura 4.I 4 são mostradas curvas experimentais de espalhamento de raios $\mathrm{X}$ para algumas composições de membranas ao longo da linha de diluição. As curvas ajustadas e seus respectivos fatores de forma e estrutura $(\mathrm{P}[\mathrm{q}]$ e $\mathrm{S}[\mathrm{q}])$ também são apresentados. $\mathrm{O}$ fator de forma otimizado pelo ajuste é muito susceptível à presença de uma região de coexistência de duas fases lamelares, o que influencia no aspecto global da curva ajustada. À medida que o Simulsol é incorporado à membrana observa-se que o fator de forma sofre alterações. Essas mudanças são mais visíveis quando se compara o perfil de contraste de densidade eletrônica $(\Delta \boldsymbol{\rho})$ para diferentes quantidades de Simulsol incorporado à membrana.

O comportamento de $\boldsymbol{\Delta} \boldsymbol{\rho}$ em função da porcentagem em massa de Simulsol na membrana é representado na figura 4. I s. Para composições da membrana com baixa concentração de Simulsol a curva $\Delta \boldsymbol{\rho}$ na região central da bicamada exibe ombros que representam certa interdigitação das caudas carbônicas (figura 4. I 5 (a)) e com o aumento da quantidade de Simulsol na membrana esses ombros desaparecem (figura 4. I $5(\mathrm{~d})$ ) tornando a curva mais suave. As moléculas de Simulsol apresentam uma única cauda carbônica e ocupam um volume menor do que as moléculas de lecitina, resultando no aumento do volume disponível para as acomodação das cadeias carbônicas.

O outro efeito da incorporação do Simulsol à membrana é visível na interface da bicamada com a água. Para membranas com baixa concentração de Simulsol observa-se um pico estreito, representando os grupos polares na superfície da bicamada, porém, com o aumento da quantidade de Simulsol esses picos tornam-se mais difusos. Esse efeito é bastante visível quando a quantidade de Simulsol na membrana é de $60 \%$ em massa (Figura 4. I $5(\mathrm{c}))$.

A incorporação de Simulsol à membrana resulta também na mudança da interface com a água devido às cadeias etoxiladas ligadas aos ácidos graxos. Na figura 4. 16 observa-se a evolução do contraste de densidade eletrônica, para duas bicamadas espaçadas de $\boldsymbol{D}$. Para membranas contendo $3 \%$ de Simulsol em massa, observa-se superposição da região dos grupos polares no regime concentrado, semelhante ao que foi observado para membrana composta de lecitina. À medida que a quantidade de Simulsol na membrana aumenta, a região polar torna-se menos definida e ocorre maior superposição das interfaces.

Barbara Bianca Gerbelli - Propriedades estruturais e elásticas de fases lamelares: O efeito da composição da membrana. 


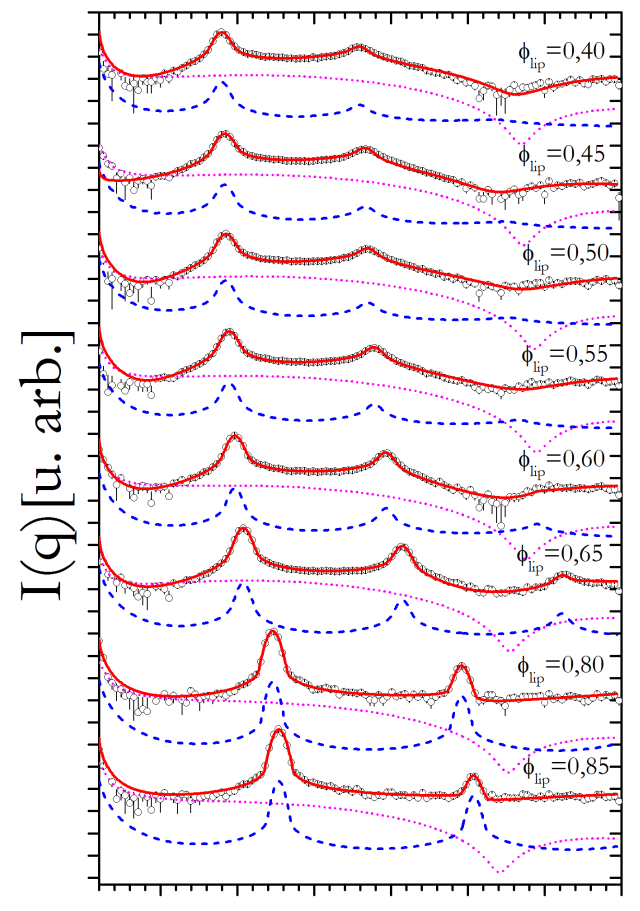

(a) $\mathbf{3 \%}$ de Simulsol

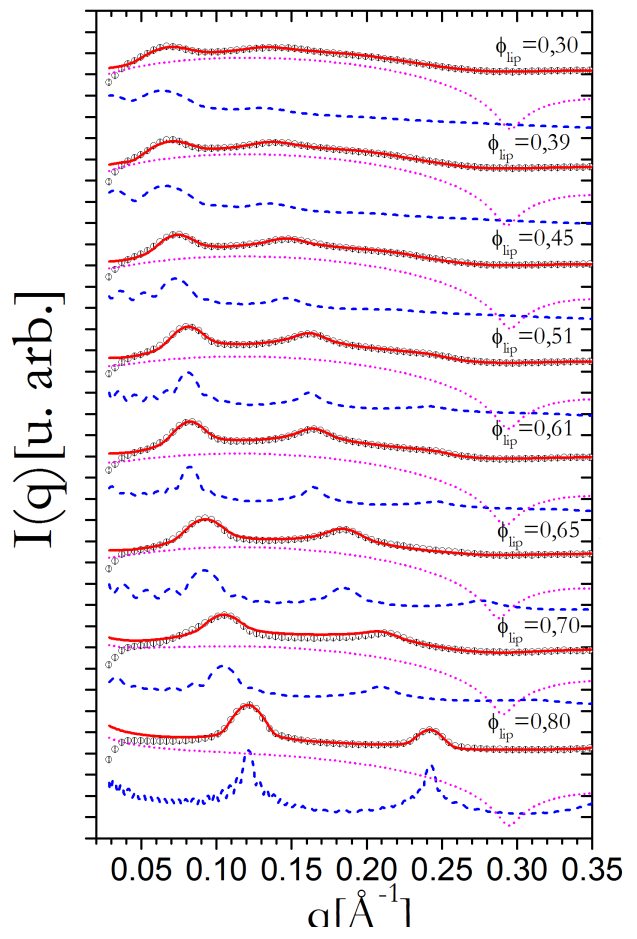

(c) $30 \%$ de Simulsol

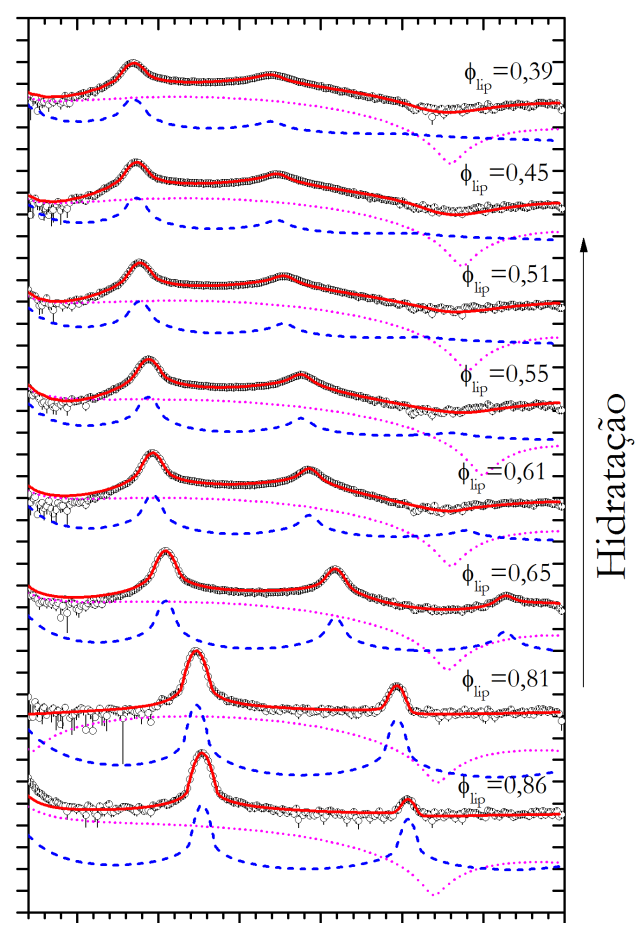

(b) $\mathbf{5 \%}$ de Simulsol

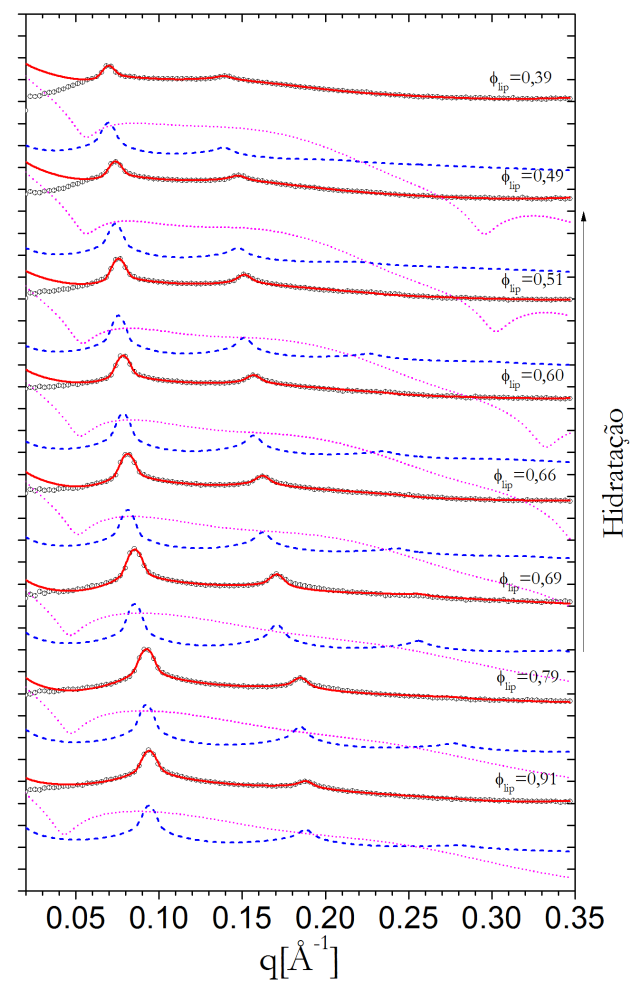

(d) $100 \%$ de Simulsol

Figura 4. I 4.: Curvas de espalhamento I(q) obtidos para fases lamelares com diferentes composições de membrana \% em massa de Simulsol. A linha cheia representa o ajuste dos dados, a linha pontilhada representa o fator de forma e a linha tracejada o fator de estrutura.

Barbara Bianca Gerbelli - Propriedades estruturais e elásticas de fases lamelares: O efeito da composição da membrana. 


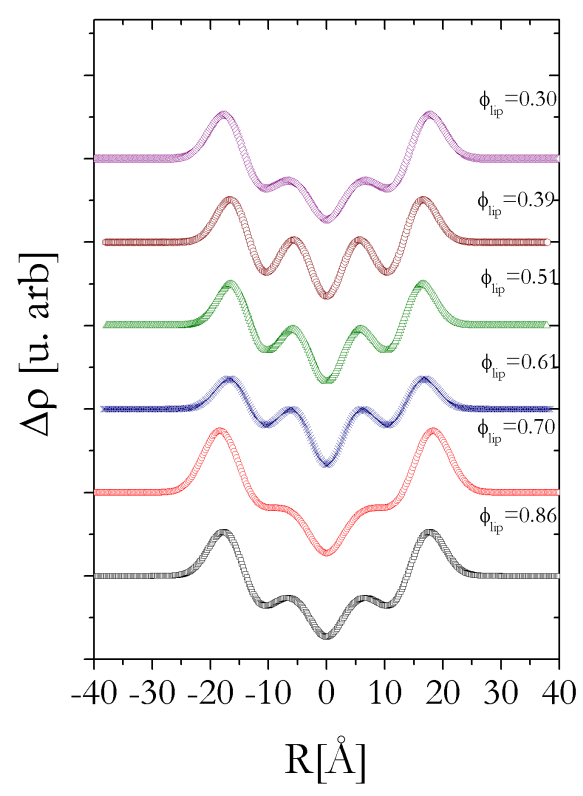

(a) $\mathbf{3 \%}$ de Simulsol em massa na membrana

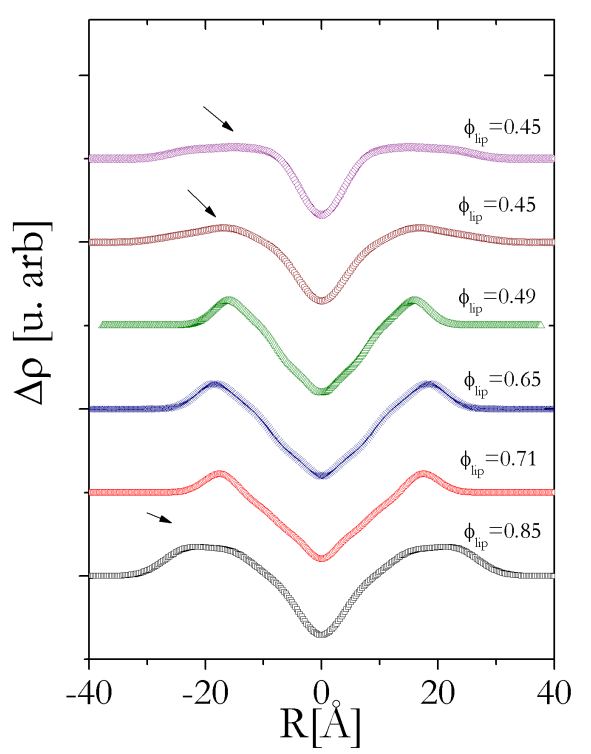

(c) $60 \%$ de Simulsol em massa na membrana

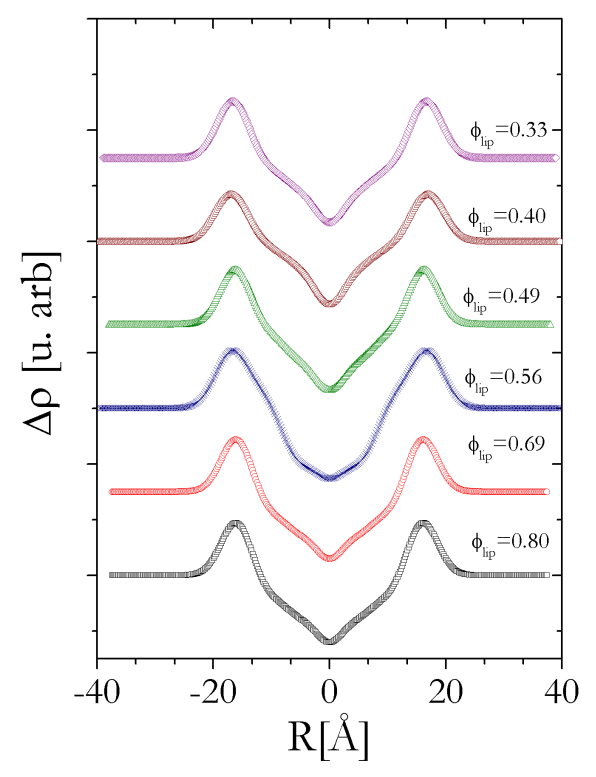

(b) $\mathbf{5 0 \%}$ de Simulsol em massa na membrana

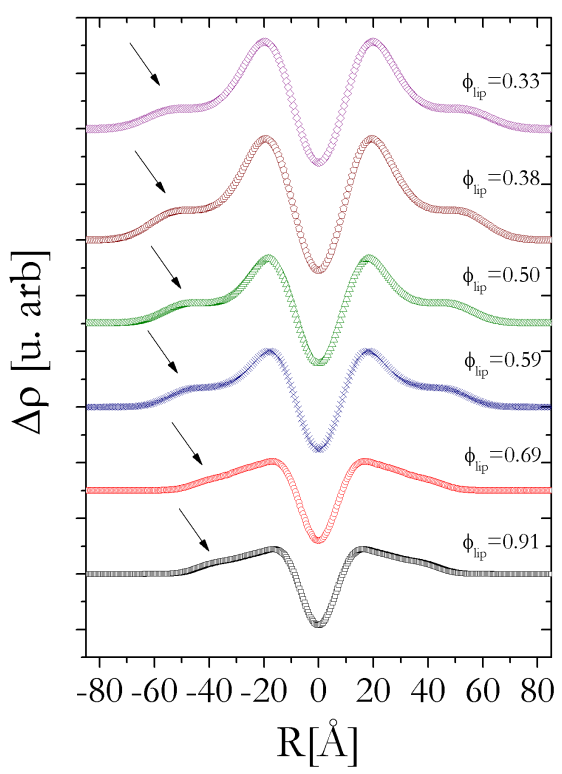

(d) $\mathbf{1 0 0 \%}$ de Simulsol em massa na membrana

Figura 4. I 5.: Contraste de densidade eletrônica para diversas composições de membrana. Nessas figuras a escala vertical de cada curva é a mesma, e as curvas são apenas deslocadas para efeito de visualização. A setas indicam o aparecimento de ombros na interface com a água.

Barbara Bianca Gerbelli - Propriedades estruturais e elásticas de fases lamelares: O efeito da composição da membrana. 


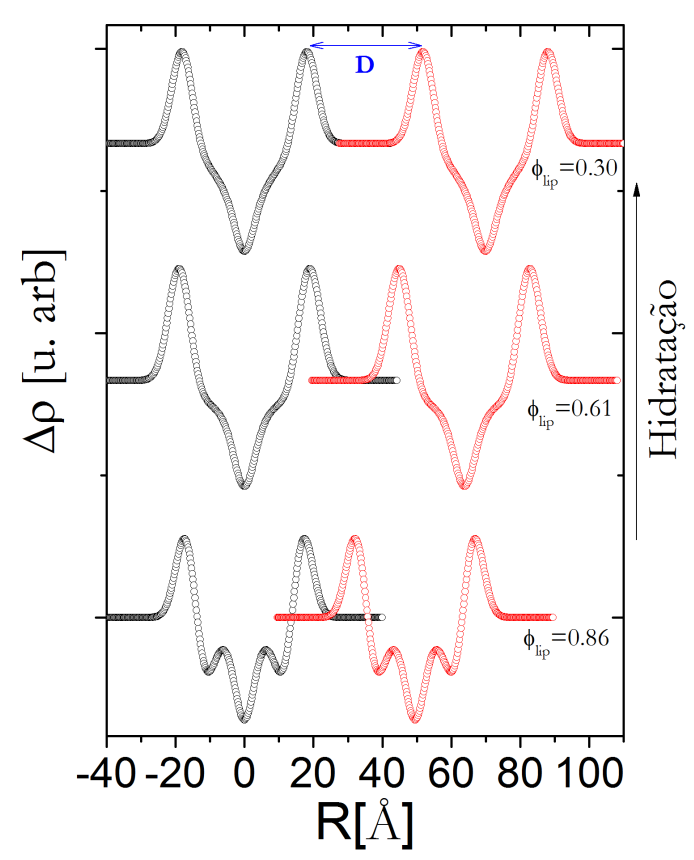

(a) $3 \%$ de Simulsol em massa

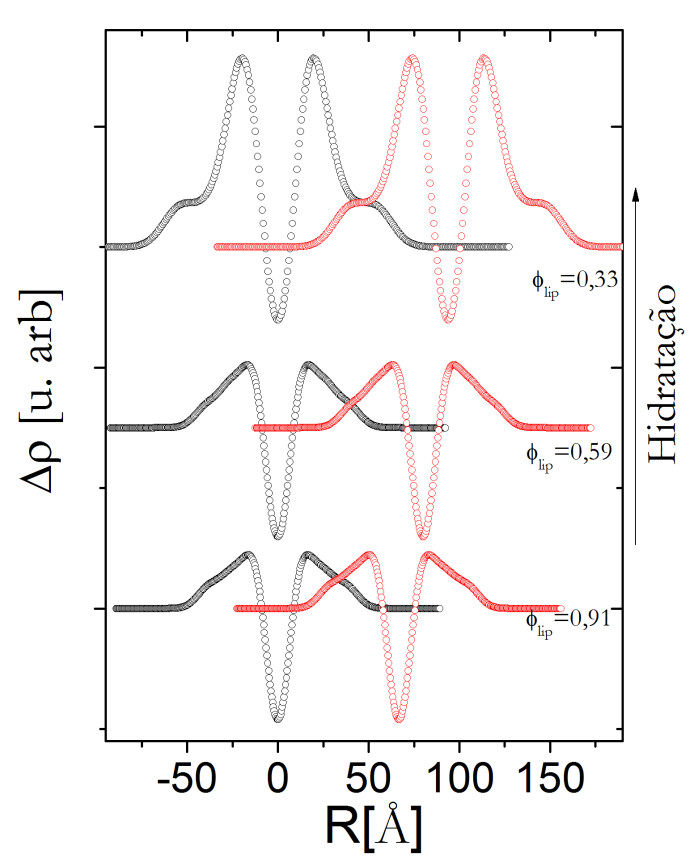

(b) $100 \%$ de Simulsol

Figura 4. I6.: Contraste de densidade eletrônica para duas bicamadas espaças de D, para duas composições de membrana.

Barbara Bianca Gerbelli - Propriedades estruturais e elásticas de fases lamelares: O efeito da composição da membrana. 
As moléculas anfifílicas de Simulsol apresentam uma cadeia de polímero hidrosolúvel ligada à cadeia carbônica. A incorporação do Simulsol modifica a interface da membrana, pois essas cadeias poliméricas se estendem para a camada aquosa podendo adquirir diferentes conformações (Figura 4.17). Para pequenas quantidades de Simulsol a conformação mais provável das cadeias poliméricas é enovelada. Devido ao efeito de volume excluído, aumentando-se a quantidade de Simulsol na membrana, a conformação mais provável da cadeia polimérica passa a ser a conformação estendida e a interface membranaágua adquire um aspecto semelhante á um tapete. Essa mudança de conformação das cadeias poliméricas na interface é conhecida na literatura como uma transição "cogumelo"escova"(mushroom-brush).

O efeito da diluição também é representado na figura (4. I7) ilustrando que para membranas ricas em Simulsol, mesmo no regime diluido, pode ocorrer a superposição das cadeias poliméricas. Quando a interface adquire esse aspecto a interface torna-se difusa e ocorre superposição das interfaces de bicamadas vizinhas.

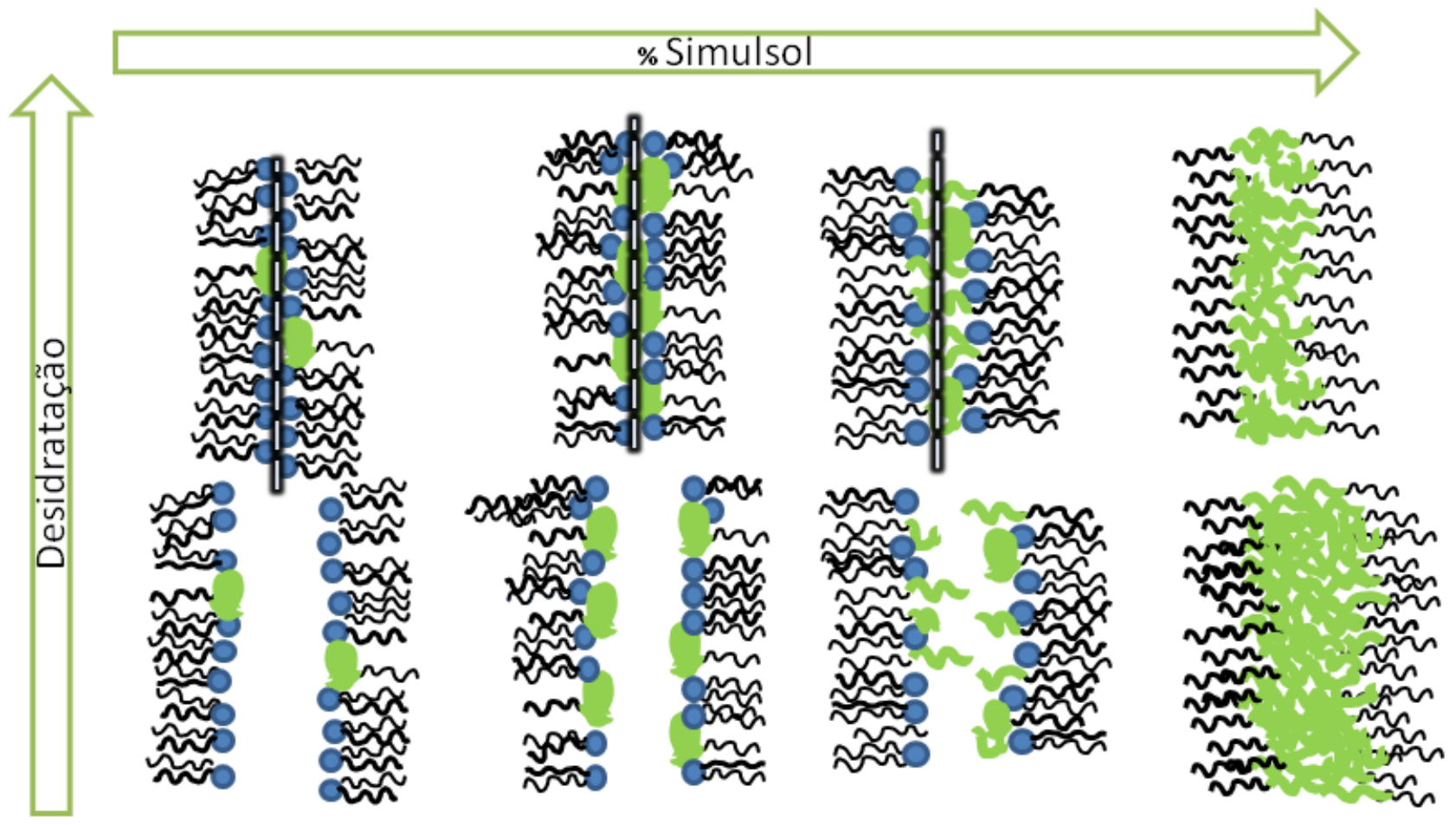

Figura 4. 17.: Representação esquemática do arranjo das moléculas anfifílicas nas bicamadas contendo Simulsol cujas moléculas apresentam uma cadeia polimérica ligada à cauda carbônica. À medida que aumenta a quantidade de Simulsol na membrana as cadeias poliméricas mudam de conformação e a interface passa ter o aspecto de um tapete.

Barbara Bianca Gerbelli - Propriedades estruturais e elásticas de fases lamelares: O efeito da composição da membrana. 
Os parâmetros estruturais obtidos nos ajustes das curvas I(q) são apresentados na figura 4. I 8 em função da quantidade de Simulsol incorporado à membrana para três frações volumétricas de lipídio; $\phi_{l i p}=0,6 ; 0,7 e 0,8$. A fração vomulétrica de lipídio 0,6 corresponde a um domínio monofásico, e para essa concentração de lipídio observamos que a periodicidade lamelar aumenta com o aumento de Simulsol na membrana, enquanto a espessura da bicamada se mantém praticamente constante em torno de $40 \AA$ (Figura 4. I $8(\mathrm{a}))$.

Para quantidades de Simulsol na membrana entre $\mathbf{1 0 \%}$ e $\mathbf{5 0 \%}$ em massa, a fração volumétrica $\phi_{l i p}=0,7$ corresponde ao domínio de coexistência de duas fases lamelares e para quantidades acima de $\mathbf{6 0 \%}$, correspondente a um domínio monofásico, com periodicidade lamelar constante. Os valores obtidos para a espessura da bicamada apresentam maior flutuação, associados à mudanças na interface membrana-água que se torna mais difusa. (Figura 4. I8(b)).

Para a fração volumétrica de lipídio $\phi_{l i p}=0,8$ o domínio bifásico ocorre para quantidades de Simulsol na membrana entre $60 \%$ e $70 \%$ em massa. No domínio mais rico em Simulsol observa-se que a periodicidade lamelar e a espessura da bicamada aumentam com o aumento da quantidade de Simulsol (Figura 4. I 8(c)).

Barbara Bianca Gerbelli - Propriedades estruturais e elásticas de fases lamelares: O efeito da composição da membrana. 


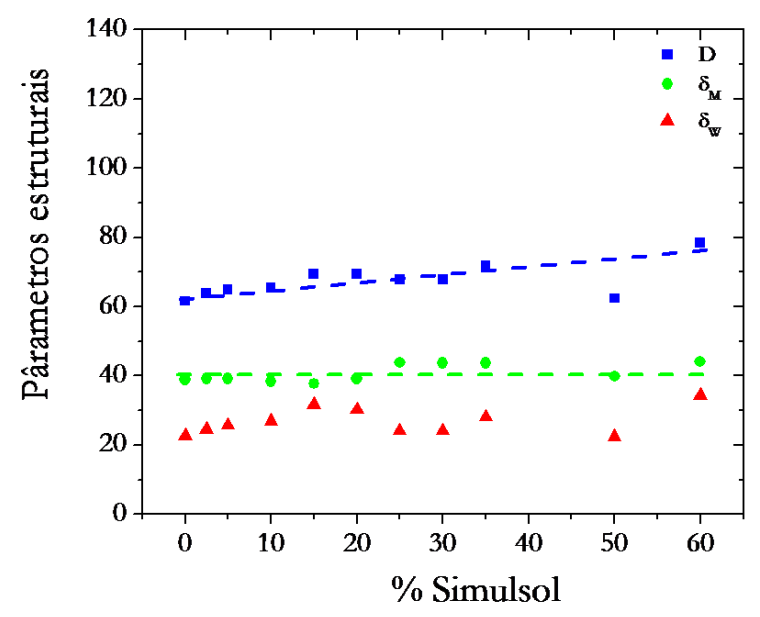

(a) $\phi_{\text {lip }}=0,6$

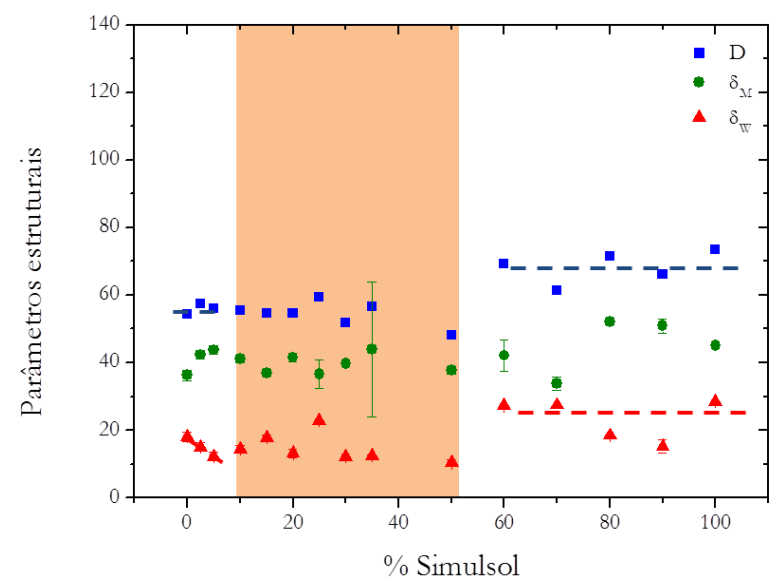

(b) $\phi_{\text {lip }}=0,7$

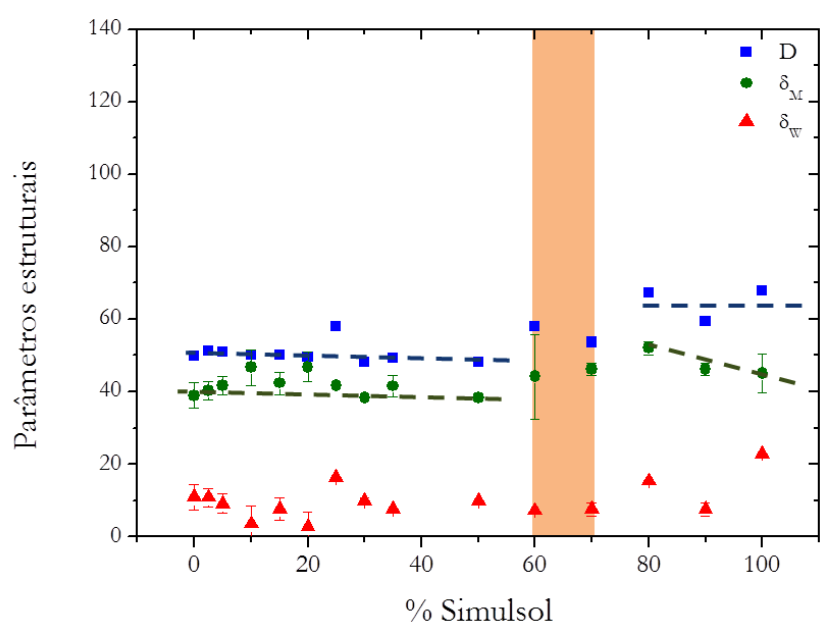

(c) $\phi_{\text {lip }}=0,8$

Figura 4. I 8.: Parâmetros geométricos obtidos através do ajuste dos dados em função de \% Simulsol em massa. A área em laranja representa o domínio de coexistência de duas fases lamelares. Na figura (b) os pontos que estão nesse domínio representam apenas uma das fases lamelares (mais concentrada).

Barbara Bianca Gerbelli - Propriedades estruturais e elásticas de fases lamelares: O efeito da composição da membrana. 
O parâmetro de Caillé é obtido do ajuste dos dados experimentais de espalhamento de raios $\mathrm{X}$ e seu comportamento para diferentes composições de membrana é mostrado na figura 4. I. Para pequenas quantidades de Simulsol na membrana, o comportamento de $\boldsymbol{\eta}$ nas regiões II e III são semelhantes ao que foi observado para membrana composta apenas de lecitina (Figura 4.r 9(a)). No domínio diluído (I), para membranas contendo Simulsol o parâmetro de Caillé apresenta um decrescimento exponencial com o aumento da fração volumétrica de lipídio, enquanto para membrana composta apenas de lecitina esse parâmetro se mantém constante.

Para membranas contendo quantidades de Simulsol maior que $\mathbf{5 0} \%$ em massa observase mudanças no comportamento do parâmetro de Caillé nos domínios II e III (Figura 4. I 9(b)) quando comparados ao comportamento da membrana composta apenas de lecitina. Nesses domínios o parâmetro de Caillé apresenta uma tendência de crescimento, que não é observada para membranas contendo quantidades menores de Simulsol.

A mudança da flexibilidade da membrana devido à incorporação do co-surfactante pode ser visualizada também pelo comportamento da amplitude média de flutuação da distância de separação entre as bicadas, $\sigma$, que é apresentado na figura 4.20. Para periodicidades até cerca de $62 \AA$ (linha pontilhada na figura 4.20(a)) $\sigma$ cresce exponencialmente seguindo o mesmo comportamento observado para membranas compostas apenas de lecitina. Com a incorporação de Simulsol à membrana abre-se um novo regime de interações onde se observa que $\boldsymbol{\sigma}$ de até $40 \AA$ A , que é comparável à espessura da bicamada.

Barbara Bianca Gerbelli - Propriedades estruturais e elásticas de fases lamelares: O efeito da composição da membrana. 


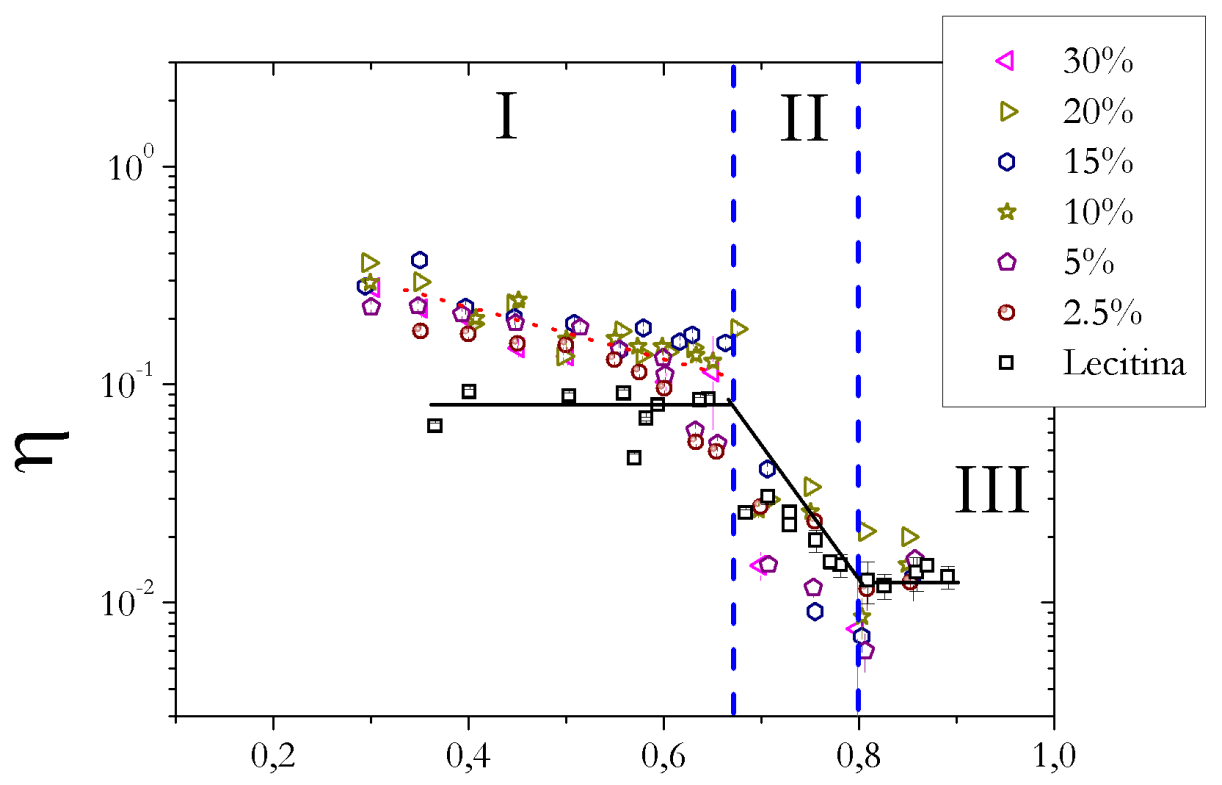

(a)

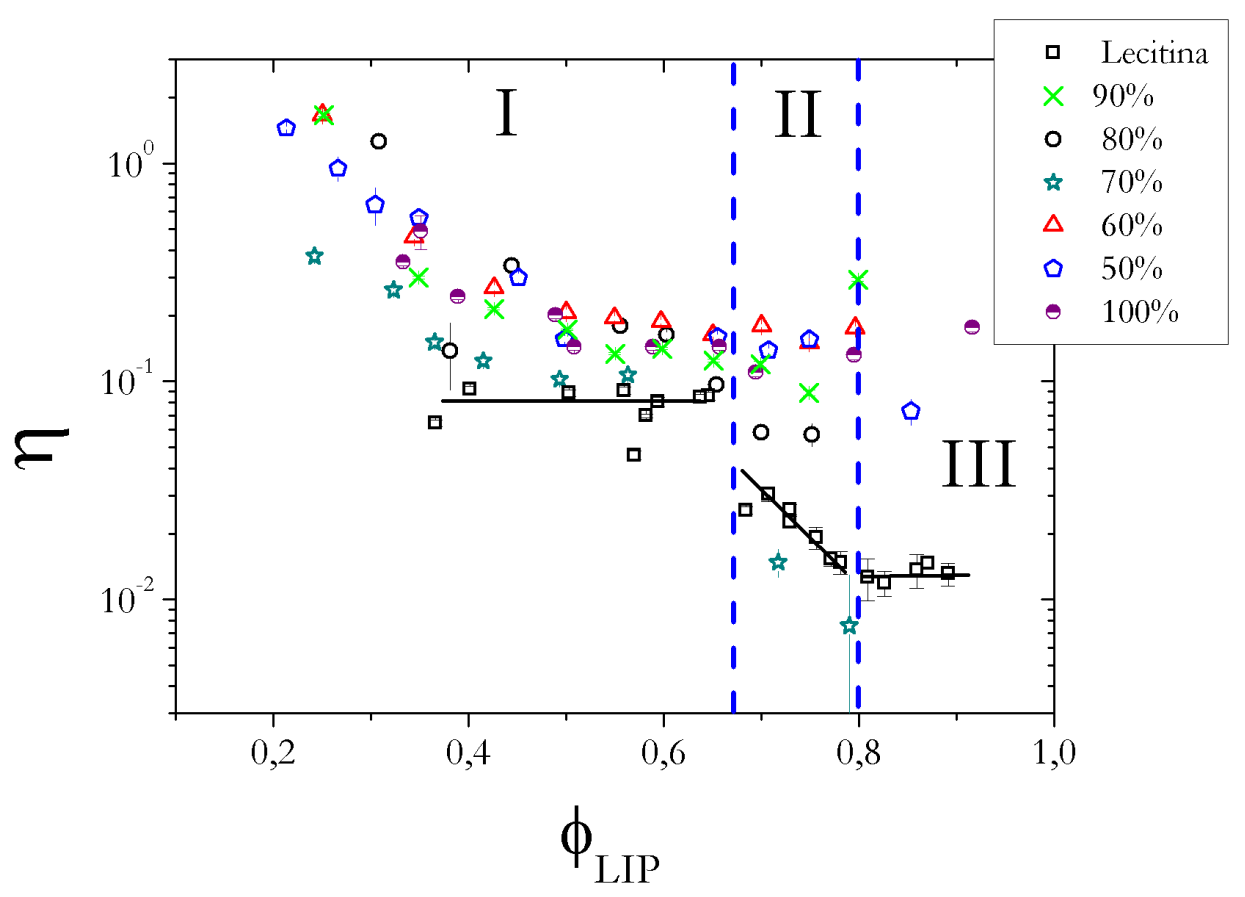

(b)

Figura 4. 19.: Comportamento do prâmetro de Caillé para diferentes quantidades de Simulsol em massa incorporados à membrana de lecitina. As linhas sólidas são apenas um guia para os olhos e as linhas tracejadas separam regiões com comportamento distintos.

Barbara Bianca Gerbelli - Propriedades estruturais e elásticas de fases lamelares: O efeito da composição da membrana. 


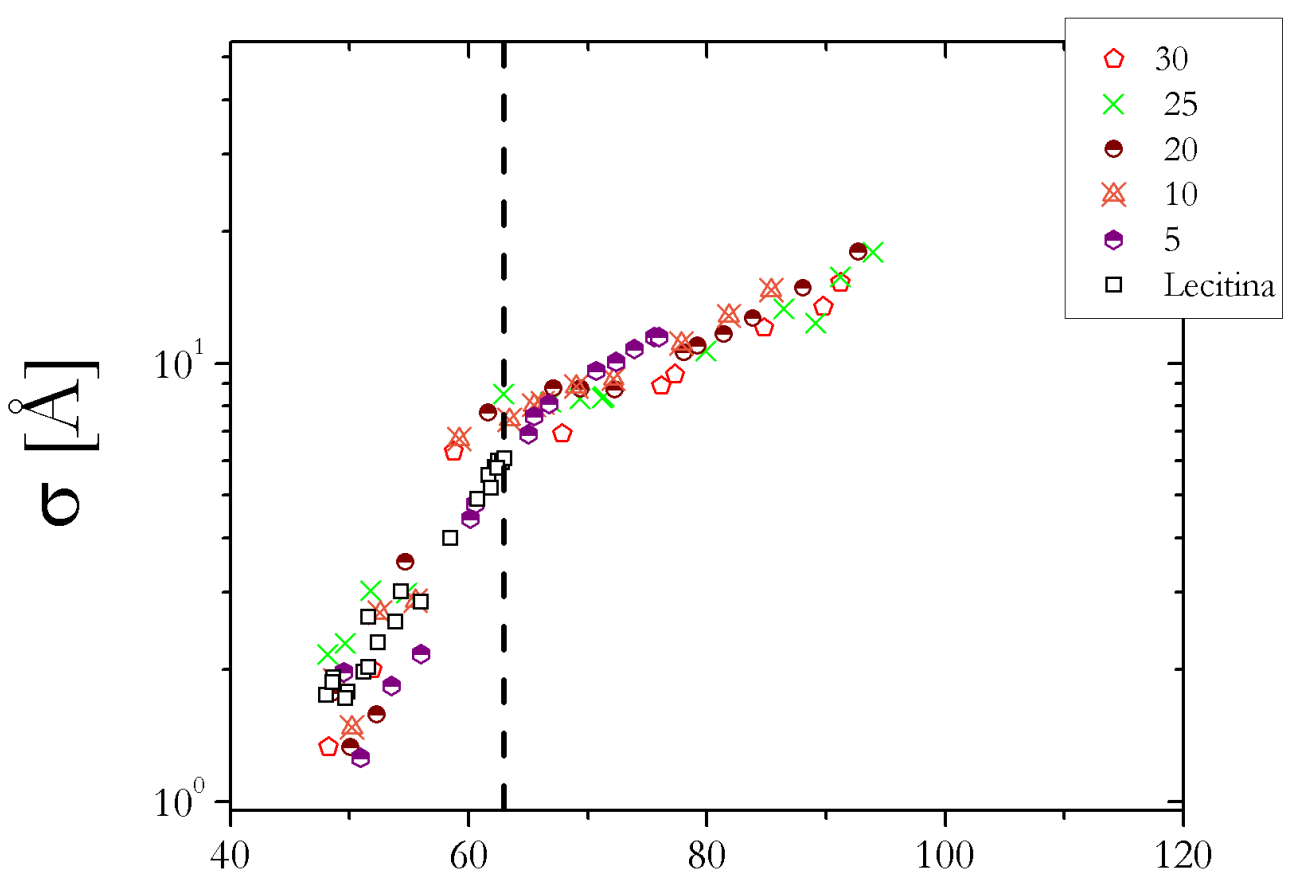

(a)

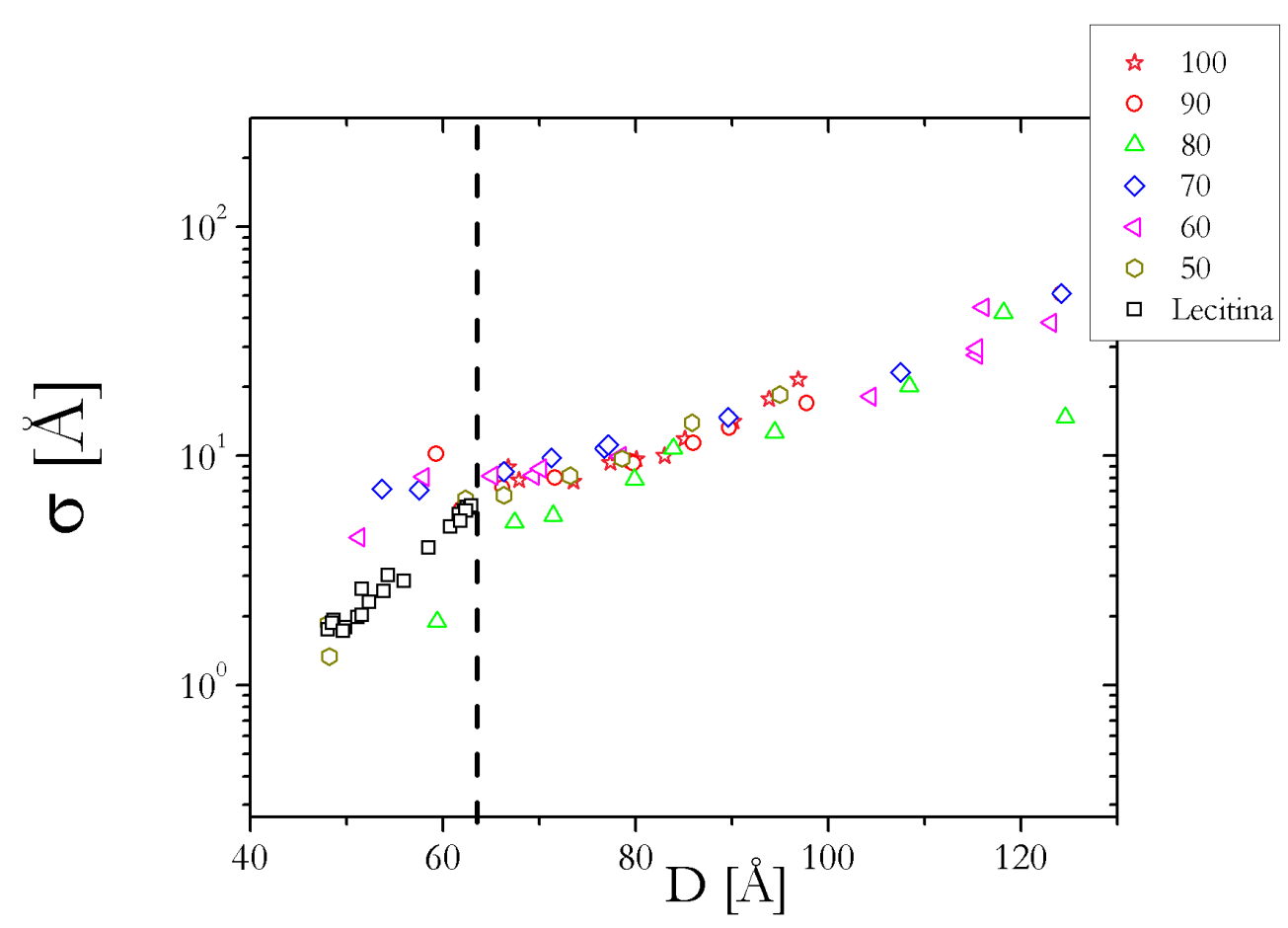

(b)

Figura 4.20.: Amplitude média de flutuação da distância de separação entre as bicamadas para diferentes quantidades de Simulsol em massa. As linhas tracejadas separam regiões com comportamento distintos de $\sigma$

Barbara Bianca Gerbelli - Propriedades estruturais e elásticas de fases lamelares: O efeito da composição da membrana. 


\section{Discussão dos resultados}

A incorporação do Simulsol à membrana promove um aumento do domínio de diluição da fase lamelar que atinge periodicidades de até 120 Å. Esse valor, no entanto, não representa uma periodicidade limite para a fase lamelar, pois não foi possível identificar claramente esse valor limite para membranas ricas em Simulsol. Observa-se também o surgimento de um domínio bifásico para $\phi_{\text {lip }}$ entre 0,7 e 0, 8 dependendo da quantidade de Simulsol na membrana.

As moléculas de Simulsol apresentam uma única cauda carbônica ligada a uma cadeia polimérica, e a presença dessas moléculas na membrana resulta em mudanças estruturais e na interface bicamada-água, ilustrada pelas curvas de perfil de contraste eletronico.

As alterações nas propriedades elásticas da fase lamelar são caracterizadas pelo comportamento do parâmetro de Caillé e da amplitude de flutuações das membranas. Observa-se que o parâmetro de Caillé tem uma tendência a decrescer quando a distância de separação entre as membranas diminui, que resulta na diminuição da amplitude de flutuação das membranas. Como o parâmetro de Caillé é inversamente proporcional a $(\mathrm{K} \overline{\boldsymbol{B}})$ a diminuição de $\boldsymbol{\eta}$ corresponde a um aumento da rigidez da membrana. De fato esse comportamento é observado para membranas compostas de lecitina e para membranas contendo baixa quantidade de Simulsol (menor que 50 \% em massa).

Para quantidades de Simulsol acima desse valor, entretanto, observa-se, que o parâmetro de Caillé apresenta uma tendência de crescimento com a diminuição da distância de separação entre as bicamadas. Esse resultado pode ser interpretado como devido à diminuição do produto $(\mathrm{K} \overline{\boldsymbol{B}})$, ou seja, o aumento da flexibilidade da membrana.

$\mathrm{Na}$ próxima seção serão apresentados resultados de experimentos que levam a determinação do módulo de compressibilidade de maneira independente. A combinação dos valores de $\overline{\boldsymbol{B}}$ obtidos combinados com os valores do parâmetro de Caillé permitem a determinação da constante de rigidez da membrana.

Barbara Bianca Gerbelli - Propriedades estruturais e elásticas de fases lamelares: O efeito da composição da membrana. 


\subsubsection{Estudo termodinâmico}

Para o estudo termodinâmico de membranas lipídicas utilizou-se a técnica de GISAXS com o controle da umidade relativa $(\mathrm{RH})$ do meio em temperatura ambiante aproximadamente $22^{\circ}$. O procedimento de preparação, bem como de calibração dos experimentos foram descritos no Capítulo 3. O processo de hidratação da fase lamelar foi investigado pela técnica de GISAXS apenas para membranas compostas de lecitina e membranas contendo $\mathbf{3 0 \%}$ de Simulsol em massa.

A figura 4.2 I apresenta os difratogramas obtidos variando a umidade relativa $(\mathrm{RH})$, onde observam-se agora pontos de Bragg, ao invés de anéis com espalhamento isotrópico.

A distância entre os pontos de Bragg são influenciadas pela umidade relativa do meio, sendo que, à medida que RH aumenta, a distância entre os picos diminui, como ilustrado pelas curvas I(q) para o filme binário de lecitina e água (figura 4.22). Observa-se que, aumentando a umidade relativa, o pico de primeira ordem se desloca para valores de $q$ menores, indicando aumento da periodicidade lamelar.

Uma das dificuldades desse experimento reside na calibração do sensor de umidade. Quando a umidade relativa se aproxima do ponto de condensação da água pode ocorrer a formação de gotas de água sobre o sensor, e a partir daí a leitura da umidade passa a ser superior a $\mathbf{1 0 0} \%$, embora a periodicidade medida para fase lamelar não tenha atingido o limite de hidratação que foi obtido para as amostras em capilares (SAXS). Verifica-se que a periodicidade ainda aumenta com o aumento do fluxo de vapor de água (Figura 4.23).

No entanto, é possível utilizar a periodicidade lamelar determinada pelo experimento de GISAXS para calibrar o sensor de umidade, combinando resultados de um experimento independente, onde a fase lamelar está submetida à pressão osmótica controlada [55]. Na figura 4.24 observa-se que a pressão descrese com o aumento da periodicidade lamelar para as duas composições de membrana estudadas. Os dados experimentais foram ajustados por uma função exponencial $\boldsymbol{P}(\boldsymbol{D})=e^{\boldsymbol{A}+\boldsymbol{B} \boldsymbol{D}}$, onde A e B são constantes arbitrárias determinadas a partir do ajuste.

A variação no potencial químico da água $\Delta \boldsymbol{\mu}$ é a energia necessária para remover I mol de água do espaço entre as membranas e transferi-la para o vapor saturado à uma dada pressão $(\mathrm{P})$ e temperatura $(\mathrm{T})$, ou seja é igual à variação da energia livre de Gibbs. A variação do potencial químico pode ser expressa como

$$
\Delta \mu=R T \ln \frac{P}{P o}
$$

Barbara Bianca Gerbelli - Propriedades estruturais e elásticas de fases lamelares: O efeito da composição da membrana. 


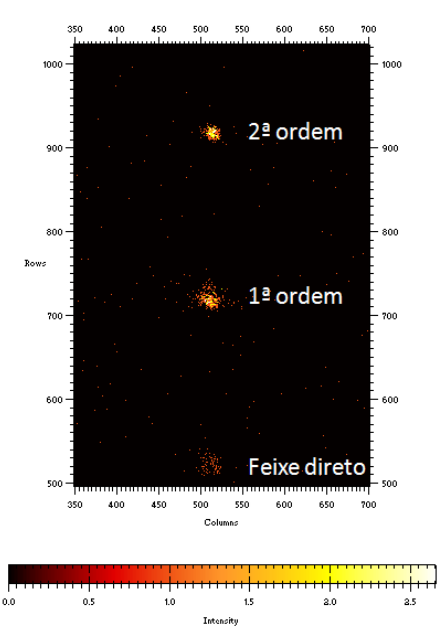

(a) $\mathrm{RH}=91,4 \%$

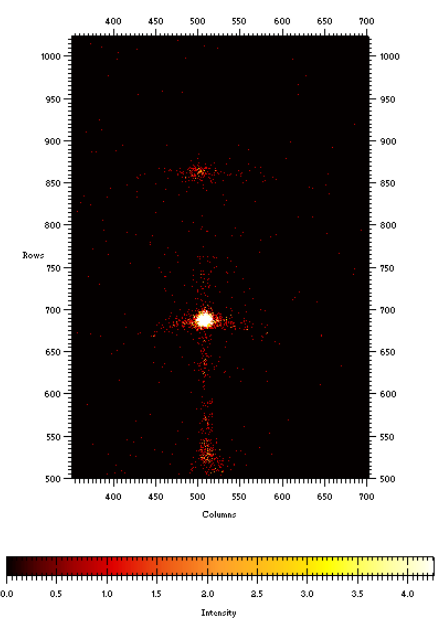

(d) $\mathrm{RH}=99,2 \%$

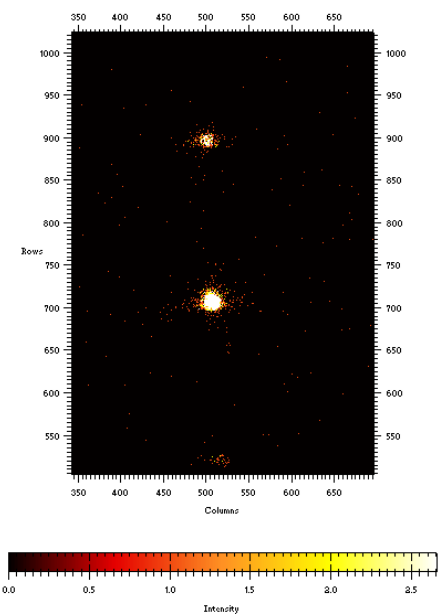

(b) $\mathrm{RH}=93,4 \%$

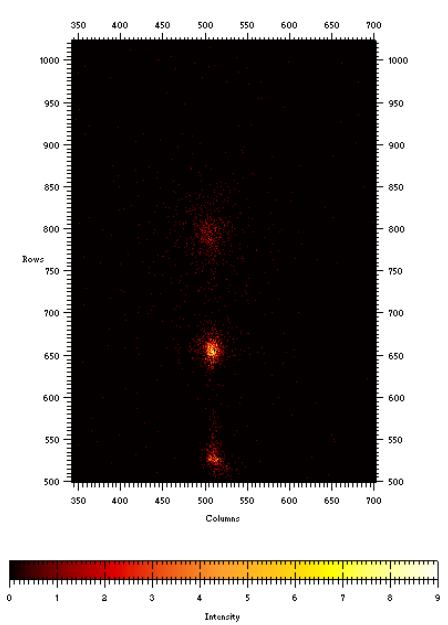

(e) $\mathrm{RH}=\mathbf{9 9}, \mathbf{8 \%}$

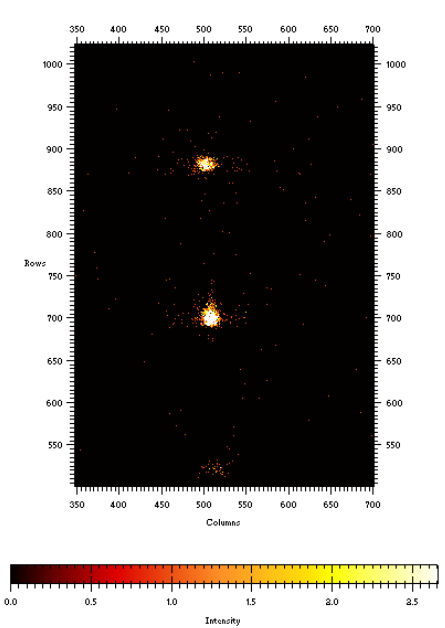

(c) $\mathrm{RH}=97,1 \%$

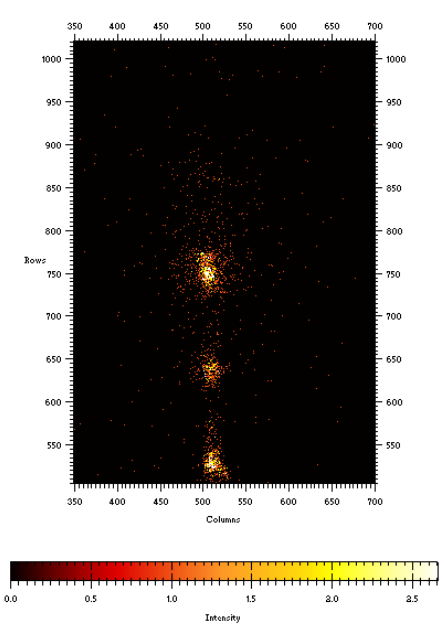

(f) $\mathrm{RH}=0,999$

Figura 4.2 I.: Difratogramas de padrões de fases lamelares para diferentes hidratações utilizando a técnica de GISAXS.

onde $\mathrm{P}$ é a pressão na água, $\boldsymbol{P}_{0}$ é a pressão de saturação do vapor, que à temperatura ambiente $\left(22^{\circ}\right)$ é igual a $2,3 \times{ }^{3} \mathrm{~Pa}$ e $\boldsymbol{R}$ é a constante universal dos gases, igual $8,21 \mathrm{~J} / \mathbf{m o l}$. A umidade relativa do meio formado pela mistura liquído+vapor saturado é expressa por:

$$
R H=100 \frac{P}{P_{0}} .
$$

Portanto a variação do potencial químico pode ser expressa em termos de $\mathrm{RH}$;

$$
\Delta \mu=R T \ln \frac{R H}{100} .
$$

Barbara Bianca Gerbelli - Propriedades estruturais e elásticas de fases lamelares: O efeito da composição da membrana. 


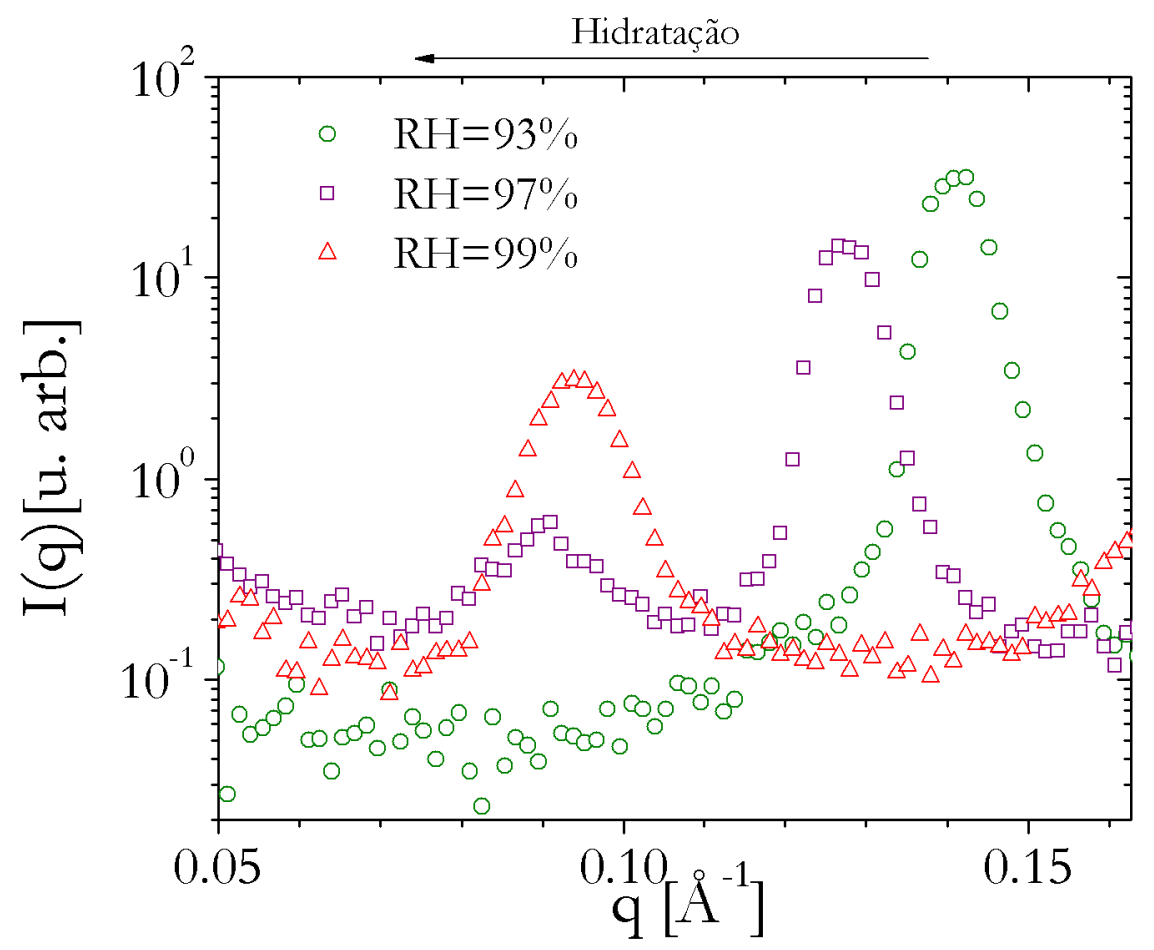

Figura 4.22.: I(q) vs. $q$ variando $\mathrm{RH}$.

Dividindo $\boldsymbol{\Delta} \boldsymbol{\mu}$ pelo volume molar parcial da água $\left(\boldsymbol{v}_{\boldsymbol{w}}\right)$;

$$
\frac{\Delta \mu}{v_{w}}=\frac{\text { energia }}{\text { mol }} \cdot \frac{\text { mol }}{\text { vol }}=\frac{\text { Forca }}{\text { Area }}
$$

tem-se a pressão diferencial aplicada à bicamada, quando uma molécula de água é removida. Assim tem-se uma expressão que relaciona a pressão exercida sobre a fase lamelar e a umidade relativa;

$$
P=\frac{R T}{v_{w}} \ln \frac{R H}{100}
$$

Portanto, para uma dada periodicidade lamelar, utilizando a equação ajustada aos dados experimentais com pressão osmótica controlada, é possível determinar a pressão correspondente a esse estado de equilíbrio (P[D]). Esse procedimento é aplicado apenas para o domínio de periodicidades lamelares comuns aos dois experimentos (pressão osmótica controlada e GISAXS). De posse do valor de P[D] obtém-se o valor correspondente da umidade relativa utilizando a equação $4.6 ; \boldsymbol{R} \boldsymbol{H}_{\text {calc }}(D)$.

Barbara Bianca Gerbelli - Propriedades estruturais e elásticas de fases lamelares: O efeito da composição da membrana. 


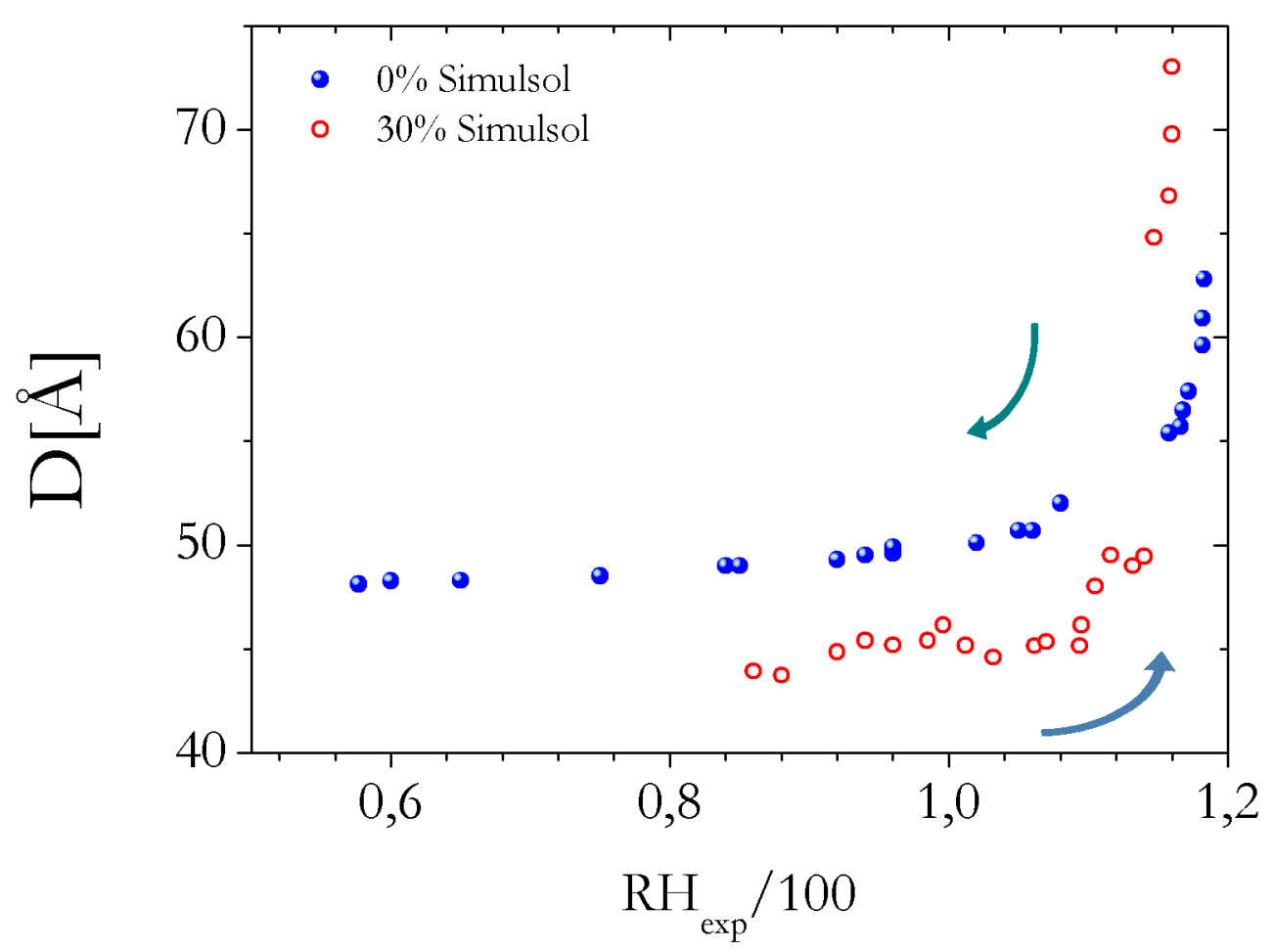

Figura 4.23.: Periodicidades lamelares obtidas por GISAXS no experimento com controle de umidade; para duas composições da membrana. As flechas na figura, indicam o processo de hidratação ou desidratação.

A calibração do sensor de umidade é feita representando em um gráfico os valores de $\boldsymbol{R} \boldsymbol{H}_{\text {calc }}(\boldsymbol{D})$ em função dos valores de $\boldsymbol{R} \boldsymbol{H}_{\text {exp }}(\boldsymbol{D})$, como mostrado na figura 4.25. Os dados experimentais são ajustados com uma função que melhor descreva o comportamento observado, fornecendo uma função $\boldsymbol{R} \boldsymbol{H}^{*}=\boldsymbol{R} \boldsymbol{H}^{*}\left(\boldsymbol{R} \boldsymbol{H}_{\text {exp }}\right)$, que permite calcular os valores de umidade relativa para todo o domínio de periodicidade lamelar varrido no experimento de GISAXS.

Barbara Bianca Gerbelli - Propriedades estruturais e elásticas de fases lamelares: O efeito da composição da membrana. 


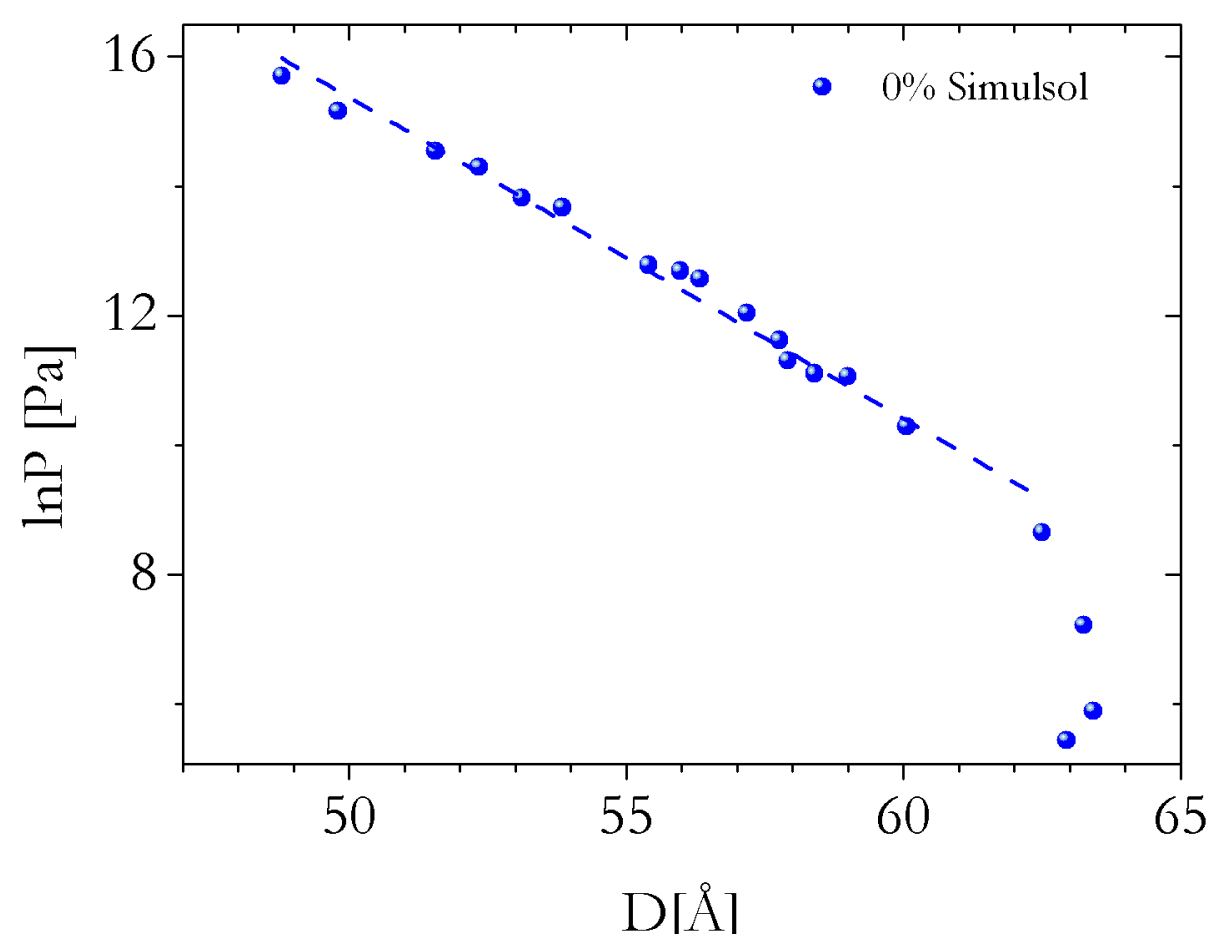

Figura 4.24.: $\ln (\mathrm{P})$, onde $\mathrm{P}$ é a pressão osmótica em função da periodicidade lamelar (Cedidos por RUBIM. R. L.).

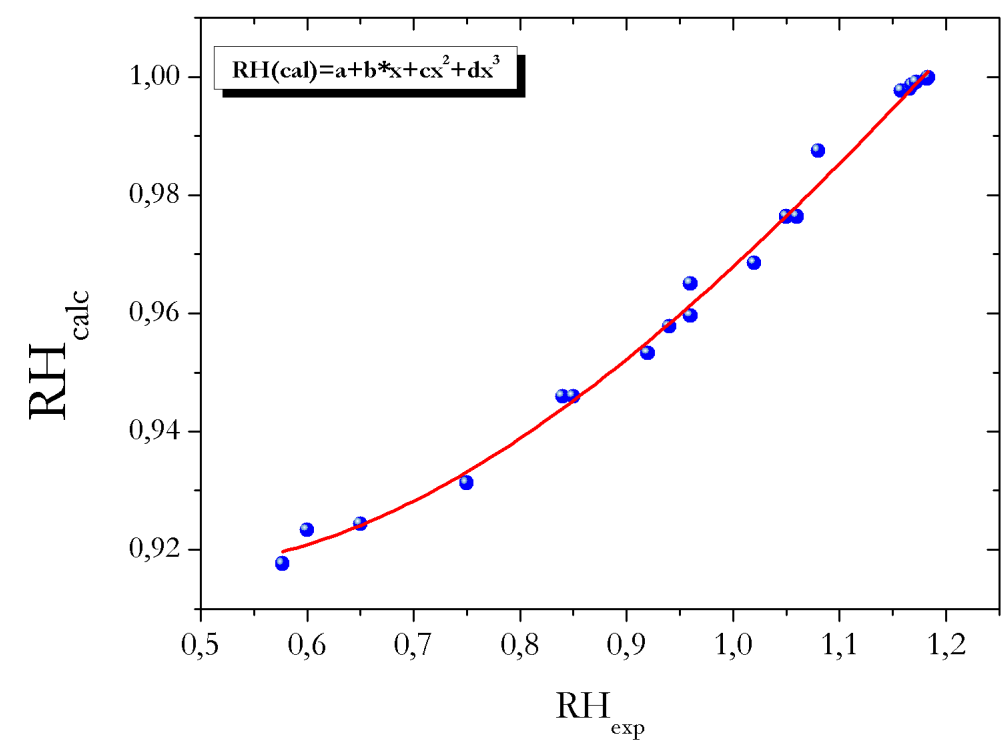

Figura 4.25.: Calibração do sensor de umidade com $\boldsymbol{R} \boldsymbol{H}_{\text {cal }}$ dado pela equação 4.6

Barbara Bianca Gerbelli - Propriedades estruturais e elásticas de fases lamelares: O efeito da composição da membrana. 


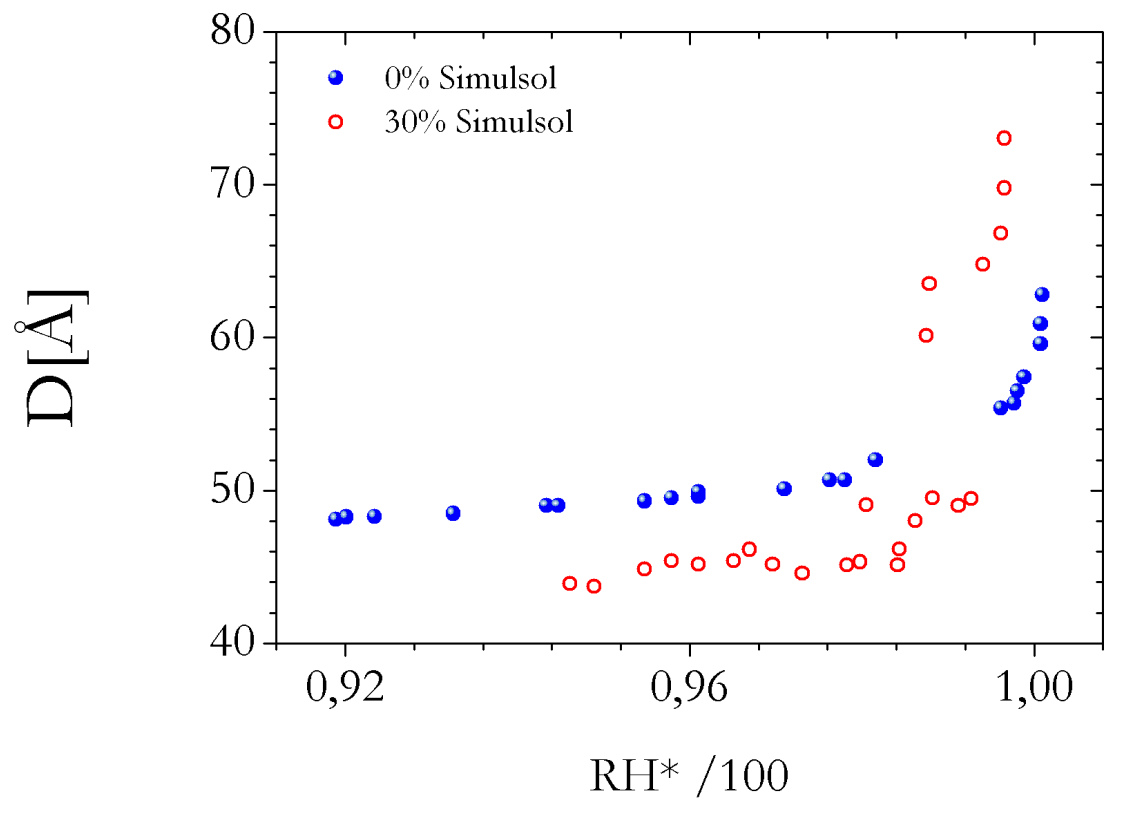

(a)

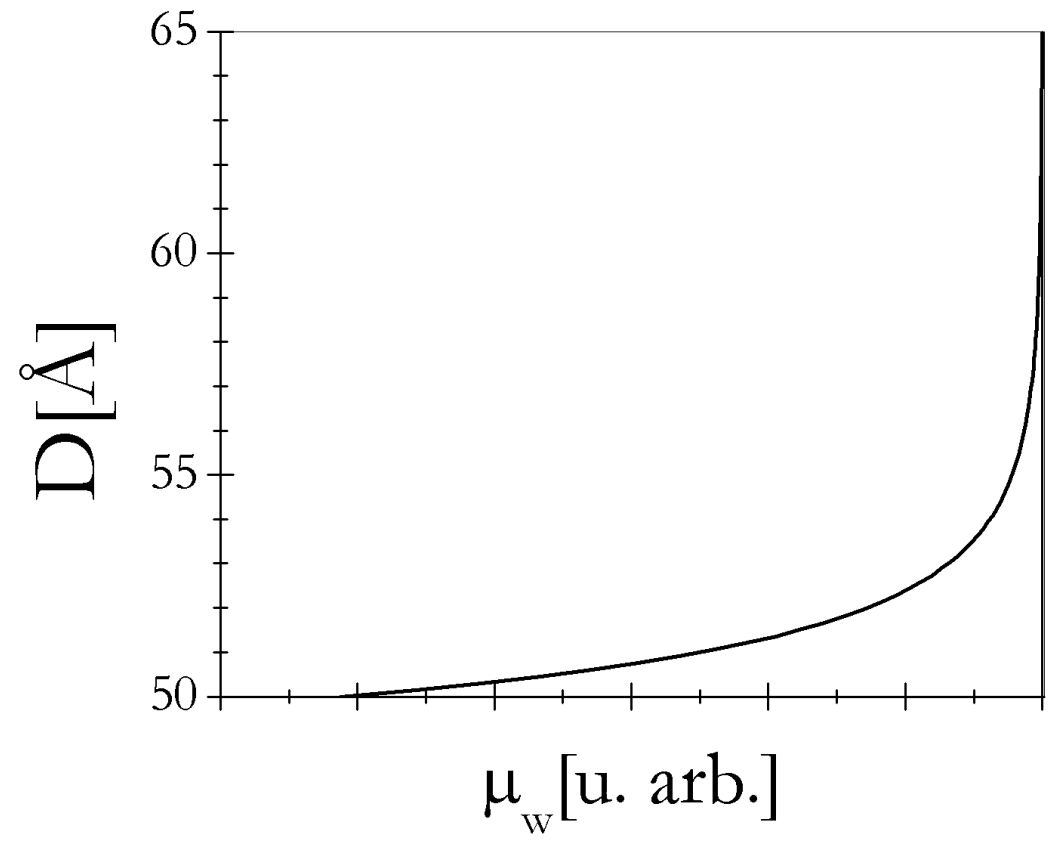

(b)

Figura 4.26.: (a) Comportamento da periodicidade lamelar determinada por experimentos de GISAXS após a calibração do sensor de umidade (b) Comportamento esperado para $\mathrm{D}$ em função do potencial químico da água $\boldsymbol{\mu}$ de acordo com a equação B. I 4.

Barbara Bianca Gerbelli - Propriedades estruturais e elásticas de fases lamelares: O efeito da composição da membrana. 
Observa-se que, grandes variações da periodicidade lamelar ocorrem somente para umidades relativas altas acima de $\mathbf{9 5 \%}$.

Para a membrana contendo $30 \%$ de Simulsol observa-se que, tanto no processo de hidratação, como no de desidratação da membrana, há um salto no intervalo de periocidades lamelares entre 50 e $60 \AA$, que corresponde ao domínio de coexistência de fase observado nos experimentos com amostras em capilares.

Esses resultados podem ser comparados com o comportamento esperado para o potencial químico da água $\left(\boldsymbol{\mu}_{\boldsymbol{w}}\right)$ na fase lamelar. A expressão abaixo foi obtida a partir de um modelo termodinâmico para fases lamelares apresentado no apêndice B [56]

$$
\mu_{s}=\frac{-8 \phi_{w}^{9}}{\left(1+\phi_{w}\right)^{9}}+\frac{C 2 \phi_{w}^{3}}{\left(1-\phi_{w}\right)^{9}}
$$

$C$ é uma constante que está relacionada à razão entre o potencial atrativo de van der Waals $\left(\boldsymbol{V}_{\boldsymbol{W}}\right)$ e o potencial repulsivo de curto alcance $\left(\boldsymbol{V}_{\boldsymbol{H}}\right)$.

Os valores de $\mathrm{RH}^{*}(\mathrm{D})$ são, então, utilizados para a determinação da pressão correspondente, que será denominada $\mathrm{P}$ *, para distingui-la da pressão osmótica obtida por outro método experimental. Na figura 4.27 é apresentado o comportamento de $\ln \mathrm{P} *$ em função da periodicidade lamelar para as duas composições estudadas Nessa figura observa-se que a pressão decresce exponencialmente com a hidratação da fase lamelar; $P^{*}(D)=P_{0} \exp [-D / \lambda], \operatorname{com} \lambda$ igual à 2 Å. Esse comportamento é compatível com resultados encontrados na literatura para fases lamelares de alguns lipídios, ilustrando o predomínio de uma interação repulsiva de curto alcance, e que tem sido denominada de força de hidratação [54]. Na mesma figura são apresentados os dados obtidos no experimento com controle da pressão osmótica com uma solução de polímero [55] para fins de comparação.

A incorporação do Simulsol à membrana modifica o comportamento de P* (figura $4.27(b))$, introduzindo dois regimes de interações entre as membranas, no entanto, ainda é possível descrever de maneira aproximada o comportamento dos dados experimentais por uma função com decaimento exponencial. Para periodicidades lamelares entre $50 \AA$ e $60 \AA$ A existe um domínio de coexistência de duas fases lamelares; uma mais diluída e uma mais concentrada. Nessa passagem pelo domínio bifásico, o potencial químico da água é igual para a fase lamelar mais concentrada e para a fase lamelar mais diluída, e isso pode ser observado na figura 4.27(b) para $\ln \boldsymbol{P}^{*} \approx 14$.

Os resultados experimentais obtidos para a membrana contendo Simulsol podem ser comparados à previsão de um modelo teórico, que considera um sistema lamelar onde a

Barbara Bianca Gerbelli - Propriedades estruturais e elásticas de fases lamelares: O efeito da composição da membrana. 
interação predominante é a interação repulsiva devido às flutuações, a partir da proposição de Helfrich. Com esse modelo é possível obter uma função analítica para $\mathrm{P}(\mathrm{D})$, que segue uma lei de potência dada por [57]:

$$
P_{D}=\frac{3 \pi^{2}}{64} \frac{\left(k_{B} T\right)^{2}}{\kappa}\left[\frac{1}{D-\delta_{m}}\right]^{3} .
$$

Na figura 4.28 são traçadas algumas curvas utilizando a expressão acima, assumindo alguns valores para a constante de rigidez da membrana. Dentro da precisão das medidas, os resultados experimentais poderiam ser descritos por duas curvas; uma para o regime concentrado, com $\kappa=1,5 k_{B} T$, e outra curva no regime diluído, com $\kappa=0,1 k_{B} T$. No entanto, esse modelo não será utilizado para a determinação do módulo de compressibilidade por duas razões; a primeira é que o modelo de Helfrich não é capaz de descrever o comportamento do parâmetro de Caillé observado, e a segunda razão, é que os perfis de densidade eletrônica obtidos mostram que a estrutura da bicamada sofre alterações com a hidratação. Portanto, é razoável supor que $\kappa$ possa também possa variar com a hidratação da membrana.

Barbara Bianca Gerbelli - Propriedades estruturais e elásticas de fases lamelares: O efeito da composição da membrana. 


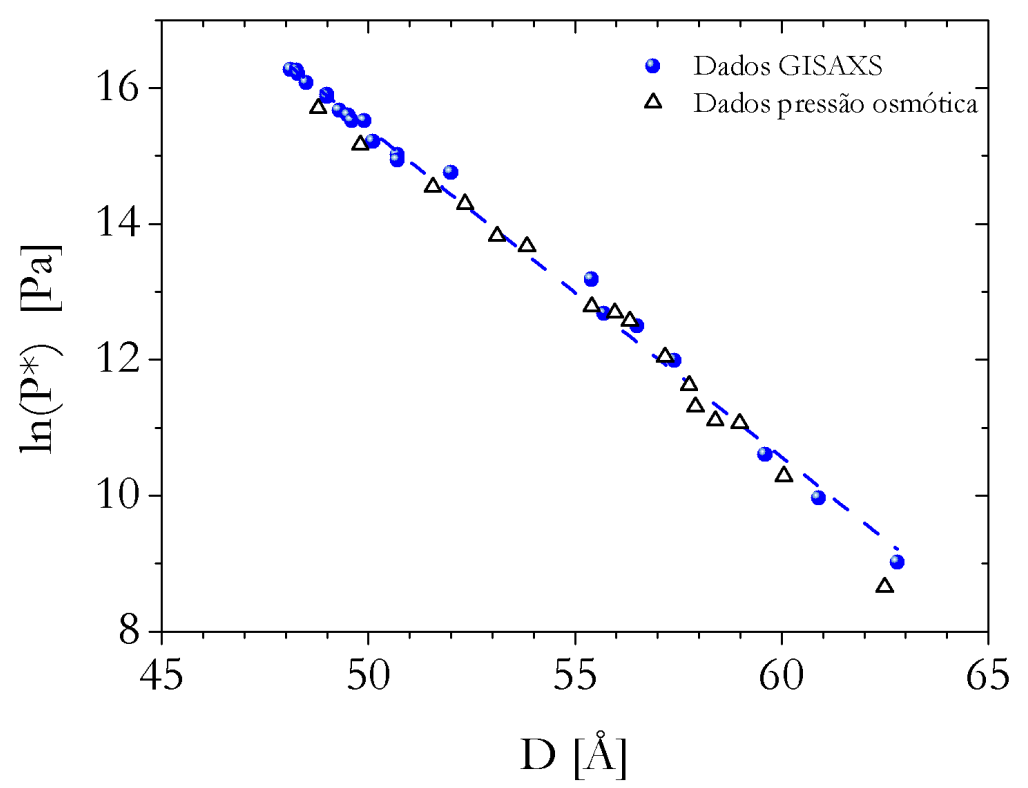

(a) $\circ \%$ de Simulsol

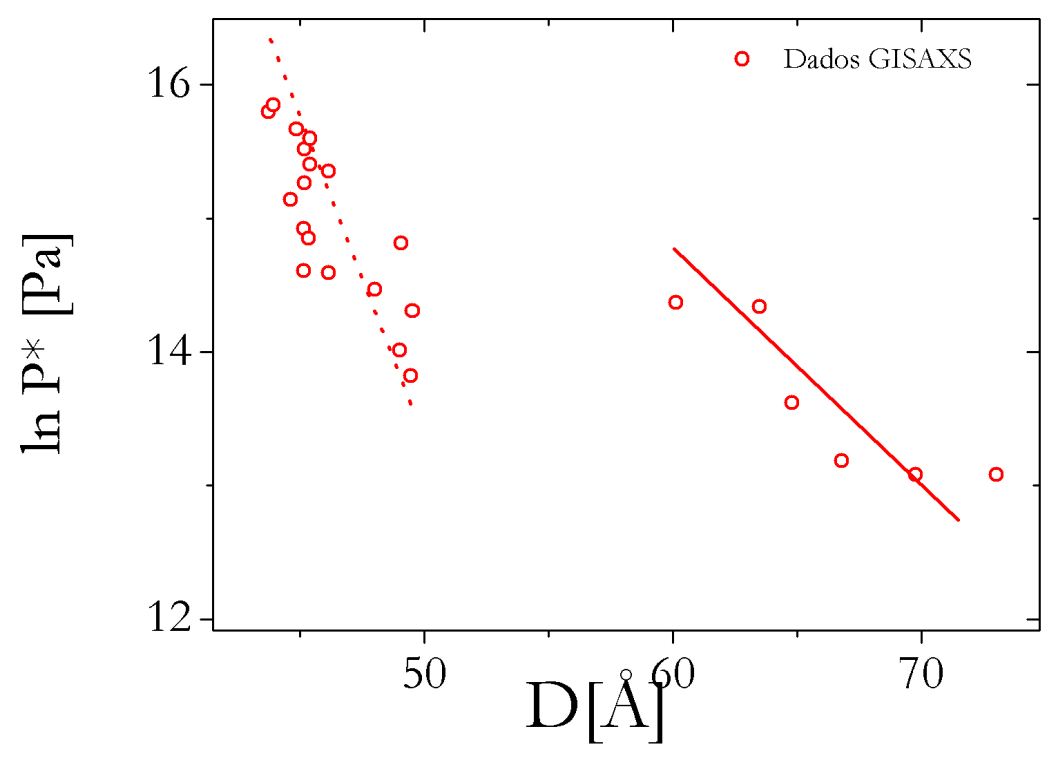

(b) $30 \%$ de Simulsol em massa

Figura 4.27.: $\ln \mathrm{P}^{*}$ em função da periodicidade lamelar (a) para as membranas de lecitina e (b) membrana contendo $30 \%$ de Simulsol em massa

Barbara Bianca Gerbelli - Propriedades estruturais e elásticas de fases lamelares: O efeito da composição da membrana. 


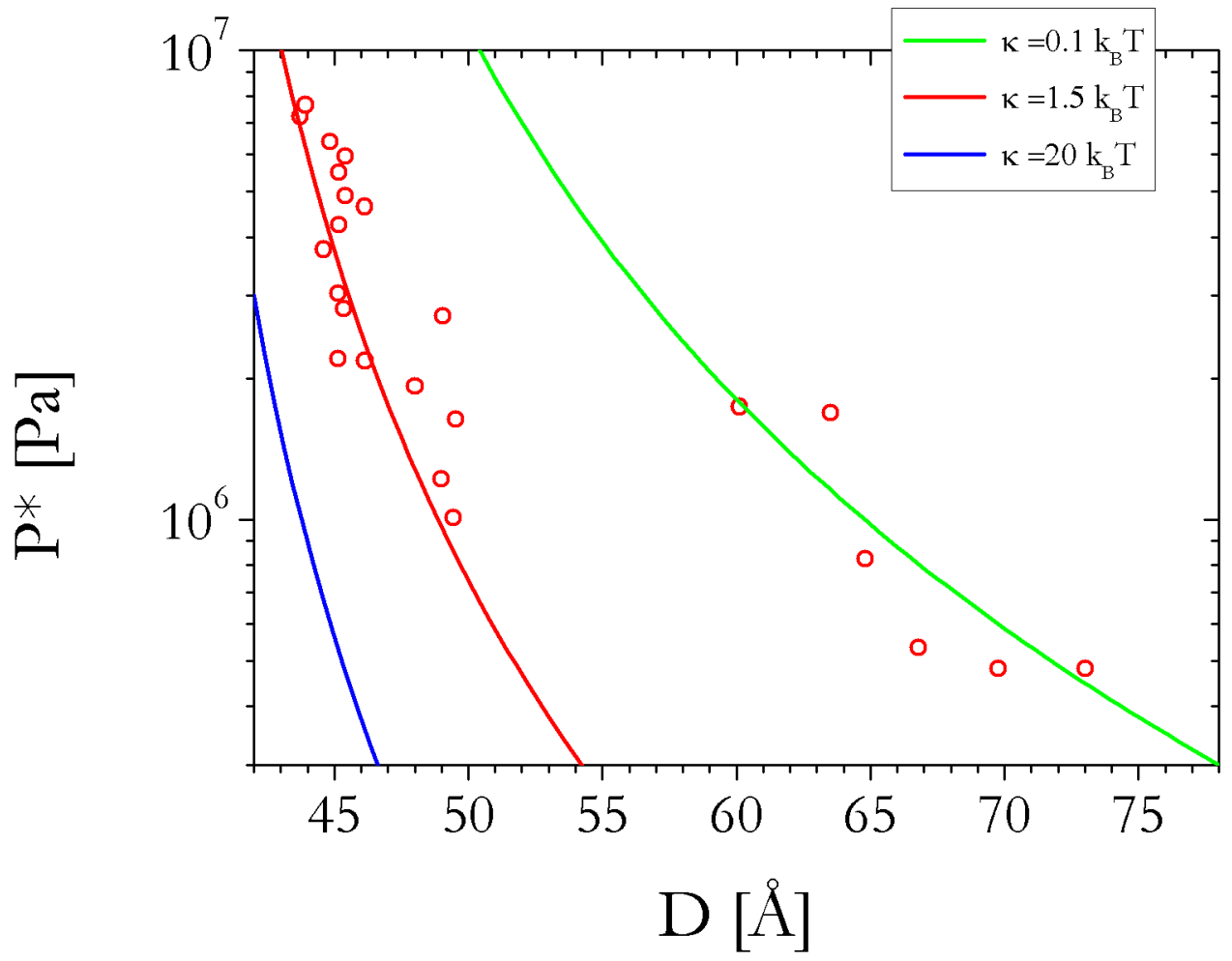

Figura 4.28.: Comparação entre os valores experimentais e valores calculados pela expressão $4 \cdot 8$. composição da membrana. 
O módulo de compressibilidade da membrana é determinado pela expressão $2.23(\bar{B}=$ $\left.-D \frac{\partial P}{\partial D}\right)$. Para o cálculo de $\boldsymbol{\partial P} / \boldsymbol{\partial D}$, os dados experimentais foram ajustados por funções exponenciais, para as duas composições de membrana estudadas. Na figura 4.29 é apresentado o comportamento de $\ln B$ em função de $D$ e, novamente, observa-se que para a membrana de lecitina os pontos experimentais estão sobre uma única reta.

O comportamento da constante de rigidez é representado na figura 4.30(b) em função da periodicidade lamelar, para as duas composições de membrana estudadas, e para ambas, observa-se, novamente, a existência de dois regimes diferentes. No primeiro regime, com periodicidade lamelar inferior a $50 \AA$, a constante de rigidez cresce exponencialmente com o aumento da hidratação da membrana, porém, a membrana contendo Simulsol é mais rígida, atingindo valores de aproximadamente $\mathbf{1 0}^{2} \boldsymbol{k}_{B} \boldsymbol{T}$. A superposição das cadeias poliméricas das moléculas de Simulsol quando as membranas estão muito próximas, poderia explicar a maior rigidez dessa membrana, em relação à membrana contendo lecitina. Os valores de $\boldsymbol{\kappa}$ são calculados utilizando os valores de $\overline{\boldsymbol{B}} / \boldsymbol{D}$ obtidos nos experimentos termodinâmicos combinados com os com valores de $\boldsymbol{\eta}$, obtidos pelo ajuste das curvas de SAXS. Na figura 4.30(a) são reapresentados os valores de $\boldsymbol{\eta}$ para a membrana de lecitina e para membrana com $\mathbf{3 0 \%}$ de Simulsol em função da periodicidade lamelar. As linhas sólidas representam ajustes de funções exponenciais aos pontos experimentais e as linhas tracejadas delimitam domínio de coexistência de fases para membrana contendo Simulsol. Para uma dada periodicidade (D) determinada no experimento de GISAXS obtém-se o valor de $\boldsymbol{\eta}$ na figura 4.30(a) e esse valor é utilizado na equação 2.9 para o cálculo de $\boldsymbol{\kappa}$.

$$
\kappa=\frac{K}{D}=\frac{B}{D}\left[\frac{{q_{0}}^{2} k_{B} T}{8 \eta}\right]^{2} .
$$

Para a membrana de lecitina, os valores de $\boldsymbol{\kappa}$ obtidos situam-se no intervalo entre $\mathbf{0 , 2}$ a $20 k_{B} T$, que são compatíveis com resultados existentes na literatura, para a lecitina e para outros lipídios ( Tabela 4. I). Parece paradoxal que a constante de rigidez aumente com o aumento da hidratação, pois a incorporação de moléculas de água no espaço entre as bicamadas contribui para o aumento da distância de separação. Nesse regime de diluição, porém, como existe uma superposição das regiões polares de bicamadas vizinhas, é possível que as moléculas de água incorporadas venham a se ligar fortemente às cabeças polares, ou no caso do Simulsol, ás cadeias carbônicas que são hidrofílicas e assim não teriam mobilidade, como no estado líquido em "bulk".

A interação entre as regiões polares das bicamadas vizinhas deve ter uma contribuição importante para pequenas distâncias de separação, pois a passagem para a situação em que

Barbara Bianca Gerbelli - Propriedades estruturais e elásticas de fases lamelares: O efeito da composição da membrana. 


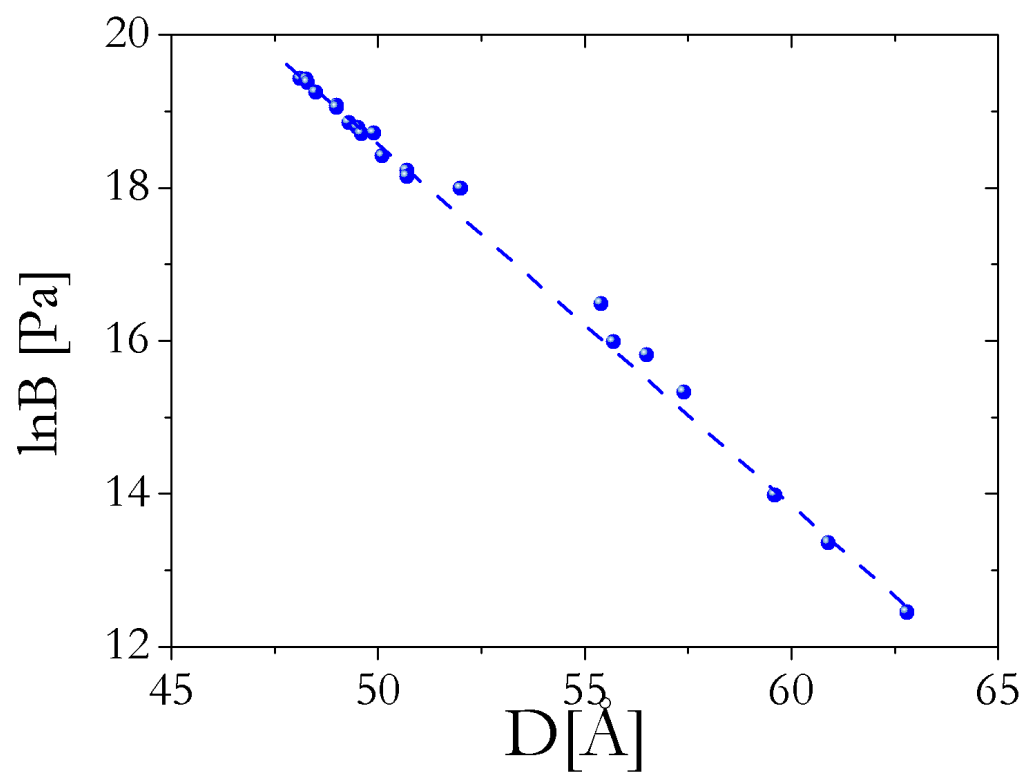

(a) ○\% de Simulsol

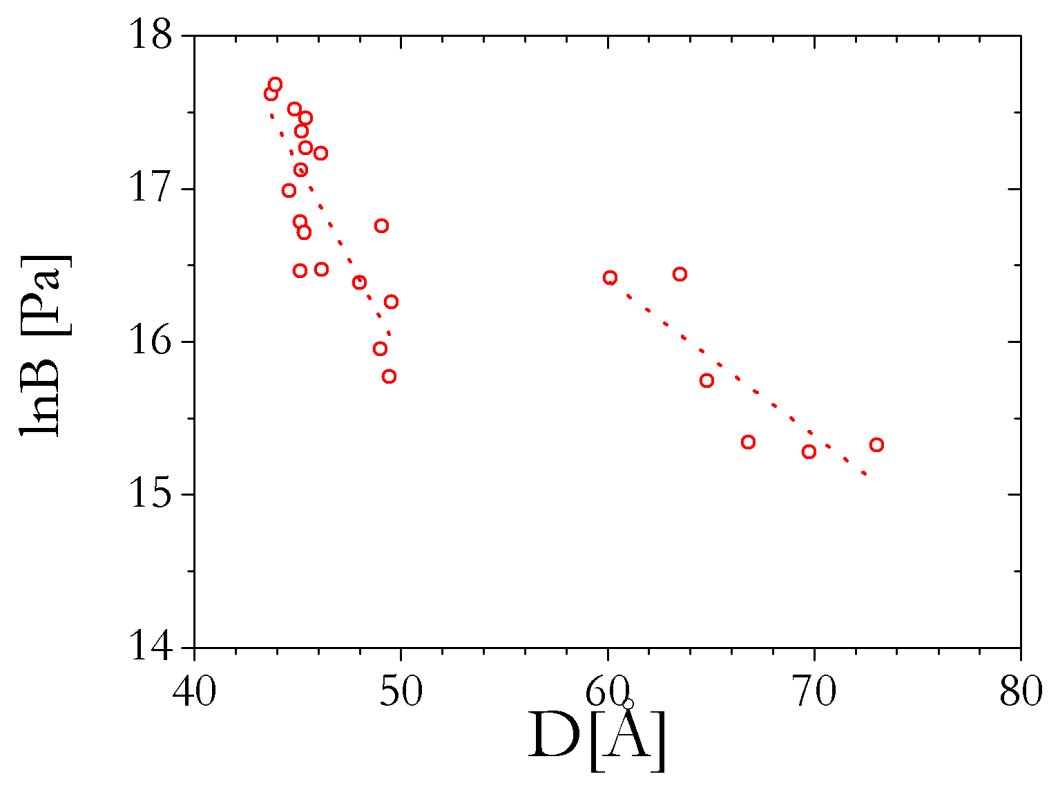

(b) $30 \%$ de Simulsol

Figura 4.29.: Módulo de compressibilidade para as duas composições de membrana estudadas.

não ocorre mais a superposição das regiões polares corresponde á mudanças no comportamento do parâmetro de Caillé e uma descontinuidade no comportamento de $\boldsymbol{\kappa}$.

Barbara Bianca Gerbelli - Propriedades estruturais e elásticas de fases lamelares: O efeito da composição da membrana. 


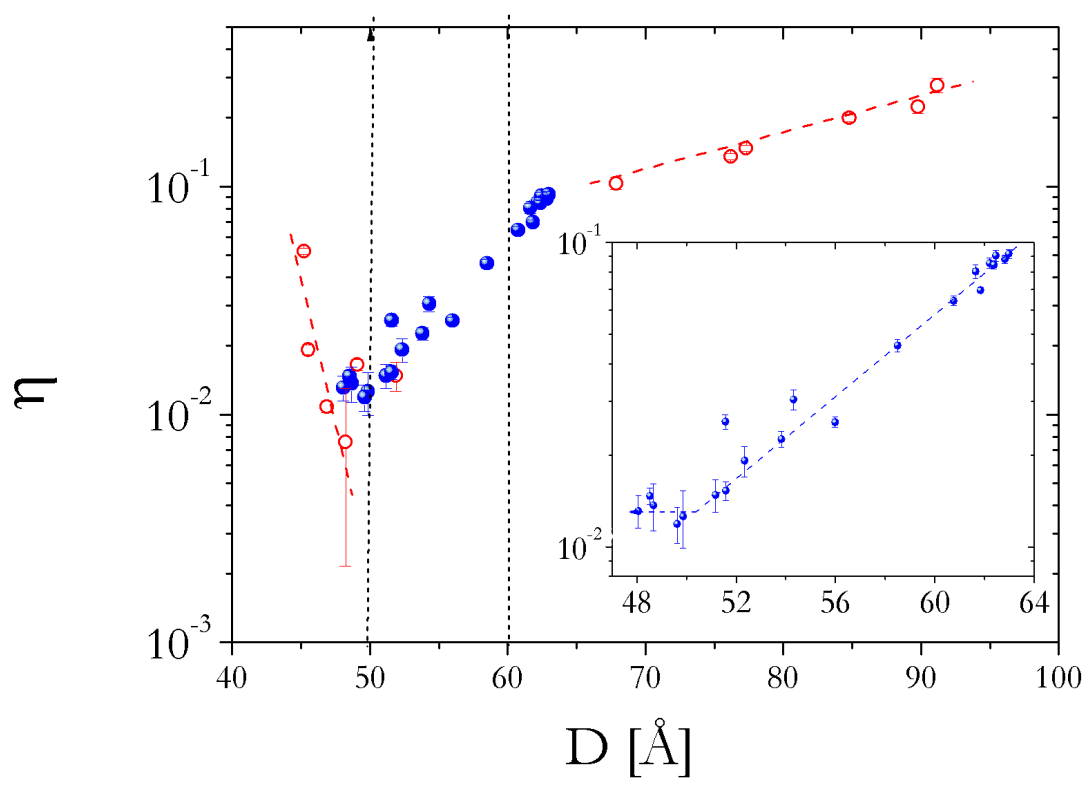

(a)

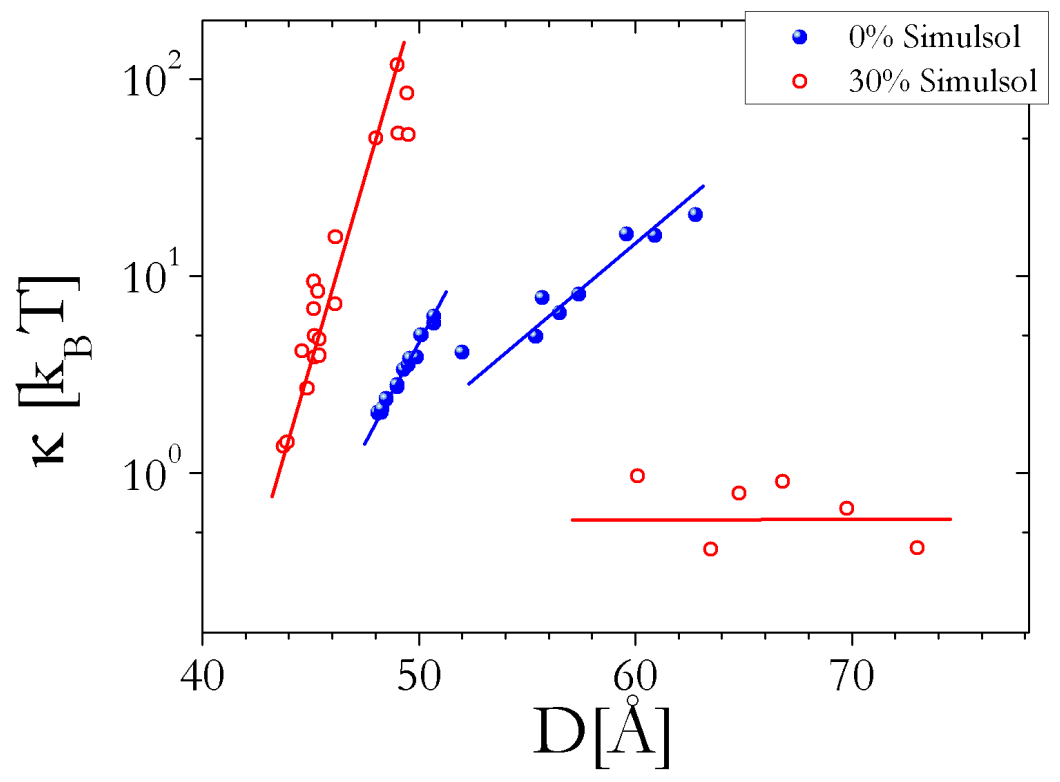

(b)

Figura 4.30.: (a) Comportamento do parâmetro de Caillé em função da periodicidade lamelar para duas composições de membranas. No quadro interno destaque para os valores obtidos para a membrana de lecitina. (b) Comportamento da constante de rigidez.

Barbara Bianca Gerbelli - Propriedades estruturais e elásticas de fases lamelares: O efeito da composição da membrana. 


\begin{tabular}{|c|c|c|c|}
\hline \hline $\boldsymbol{\kappa}\left(\boldsymbol{k}_{\boldsymbol{B}} \boldsymbol{T}\right)$ & Técnica & Referência & Lipídio \\
\hline \hline $\mathbf{1 5}-\mathbf{2 0}$ & SAXS & {$[23]$} & DOPE \\
\hline$\sim \mathbf{8}$ & SAXS/MLV & {$[\mathbf{2 2}]$} & DOPE \\
\hline $\mathbf{2 0}$ & SAXS & {$[\mathbf{2 2}]$} & Lecitina de ovo \\
\hline
\end{tabular}

Tabela 4. I.: Valores de constante de rigidez para membranas disponíveis na literatura.

A constante de rigidez da membrana contendo Simulsol, com periodicidade lamelar entre 60 e $80 \AA$ é aproximadamente constante $\approx 0,5 k_{B} \boldsymbol{T}$. Nesse regime, embora possa ocorrer superposição das cadeias poliméricas na camada aquosa, a presença das moléculas de Simulsol na membrana confere maior flexibilidade á membrana, pois essas moléculas ocupam um volume menor comparado às moléculas de lecitina. Esse valor é da mesma ordem de grandeza da constante de rigidez de membranas de DMPC e pentanol [49], e, para esse sistema lamelar o comportamento do parâmetro de Caillé segue o comportamento previsto pelo modelo de Helfrich, (figura 2. I 2), indicando a predominância de interações estéricas devido às flutuações da membrana. Para esse regime, a quantidade $\left[\mathbf{1}-\boldsymbol{\delta}_{m} / \boldsymbol{D}\right]^{2}$, que aparece na figura 2. I 2 corresponde a valores entre 0,12 e 0,25 da membrana contendo $30 \%$ de Simulsol em massa, portanto, um intervalo ainda muito pequeno para confirmar a previsão do modelo de Helfrich. Experimentos de SAXS, explorando o regime diluído até o limite de diluição, se ele existe, podem ser esclarecedores.

Barbara Bianca Gerbelli - Propriedades estruturais e elásticas de fases lamelares: O efeito da composição da membrana. 


\section{Discussão dos resultados}

Os experimentos realizados com filmes orientados em um ambiente com umidade controlada permitiram o estudo do processo cinético de hidratação e desidratação da fase lamelar para duas composições de membrana; apenas de lecitina e contendo $\mathbf{3 0} \%$ de Simulsol em massa. A dificuldade técnica de medir umidade relativa muito alta foi contornada, utilizando resultados de um experimento independente, com controle da pressão osmótica, que permitiram a calibração do sensor de umidade. Com esses experimentos tem-se acesso experimental á variação do potencial químico permitindo a investigação de propriedades termodinâmicas do sistema lamelar. A combinação do experimento termodinâmico com estudos estruturais da fase lamelar permite a determinação das constantes elásticas que caracterizam a flexibilidade das membranas; o módulo de compressibilidade e a constante de rigidez.

O aumento do período lamelar com o aumento da umidade relativa mostra um comportamento qualitativo compatível com o comportamento esperado para o potencial químico da água em uma fase lamelar e revela que, de fato, é preciso que a umidade relativa seja bastante alta, para que as variações no período lamelar sejam observadas. A coexistência das duas fases lamelares, identificado para a membrana contendo Simulsol nos experimentos de SAXS, é observada claramente no experimento de GISAXS, pelo salto da periodicidade lamelar para um dado valor da pressão osmótica (ou potencial químico).

É interessante notar que, no regime concentrado $(\boldsymbol{D}<50 \AA$ Å), o módulo de compressibilidade da fase lamelar contendo Simulsol é menor do que o da fase lamelar de lecitina, porém a constante de rigidez da membrana é maior. A constante de rigidez da membrana está relacionada às flutuações de ondulações e o aumento observado pode ser atribuído à superposição das cadeias poliméricas na camada aquosa entre as bicamadas, como mostrado na figura 4.20. Por outro lado, o módulo de compressibilidade está relacionado às flutuações na distância de separação entre as membranas (fig. 2.7), e a diminuição no valor de $\overline{\boldsymbol{B}}$ com a incorporação de Simulsol significa que a camada aquosa contendo algumas cadeias de polímero é mais fluida do que a camada aquosa entre as moléculas de fosfolipídios. O aumento de fluidez da camada aquosa poderia ser atribuído, então, à presença das cadeias poliméricas, que inibem a organização das moléculas de água.

A constante de rigidez da membrana de lecitina, no regime diluído, é reduzida de maneira significativa com a incorporação de Simulsol e esse efeito pode ser interpretado pela redução do volume ocupado pelas moléculas de Simulsol, que possuem uma cadeia carbônica. É difícil, no entanto, explicar que a constante de rigidez da membrana de lecitina continue a aumentar, com o aumento da distância de separação entre as membranas.

Barbara Bianca Gerbelli - Propriedades estruturais e elásticas de fases lamelares: O efeito da composição da membrana. 
Até o momento, os modelos teóricos que descrevem as interações entre as membranas não são adequados para descrever os resultados obtidos. Embora a interação eletrostática devido à presença da dupla camada de íons e contra-íons na interface da bicamada não esteja presente no sistema lamelar estudado, interações dipolares entre os grupos fosfatos presentes na cabeça polar das moléculas anfifílicas entre esses grupos e as moléculas de água, sobretudo no regime confinado, para o qual a distância de separação entre as bicamadas é da ordem de $5 \AA$ e $8 \AA$.

Barbara Bianca Gerbelli - Propriedades estruturais e elásticas de fases lamelares: O efeito da composição da membrana. 


\section{CAPÍTULO 5}

\section{CONCLUSÕES E PERSPECTIVAS}

\subsection{Conclusões}

Neste trabalho foi realizado um estudo das propriedades estruturais e elásticas de uma fase lamelar de lipídios. Os aspectos inovadores deste trabalho residem primeiro, na abordagem experimental que consiste na modificação sistemática da composição da membrana para investigar o papel dos mecanismos entrópicos (volume excluído) para a estabilização da fase lamelar. O segundo aspecto está na aplicação de um método global para a análise dos dados experimentais de espalhamento de raios $\mathrm{X}$ que permitem a determinação do perfil de densidade eletrônica e do fator de estrutura. A partir dessa análise é possível descrever mudanças importantes que ocorrem na interface entre a bicamada-água que dependem da composição da membrana. Finalmente, o terceiro aspecto consiste no desenvolvimento de uma montagem para experimentos de espalhamento de raios $\mathrm{X}$ com controle de umidade, adaptada ao NanoStar. Esses experimentos permitiram o estudo cinético do processo de hidratação-desidratação de maneira contínua explorando o comportamento termodinâmico da fase lamelar.

Os experimentos de espalhamento de raios X à baixo ângulo (SAXS) com análise global dos dados permitem a determinação do parâmetro de Caillé, que caracteriza as flutuações das membranas, assim como parâmetros estruturais. A incorporação de Simulsol à membrana aumenta o domínio de diluição e periodicidades da ordem de 120 Å são observadas. Para algumas composições de membrana, mais ricas em Simulsol, não está claro se existe uma periodicidade limite. Esse é um aspecto que será investigado no futuro. Outro efeito do co-surfactante na membrana são as mudanças estruturais na bicamada e na interface

Barbara Bianca Gerbelli - Propriedades estruturais e elásticas de fases lamelares: O efeito da composição da membrana. 
que se torna mais difusa.

O comportamento do parâmetro de Caillé revela que mudanças na composição da membrana abrem um novo regime de interação entre as membranas, que não era acessível para a fase lamelar de lecitina. Os modelos disponíveis na literatura que descrevem o comportamento do parâmetro de Caillé são adequados apenas para duas situações limites; quando a interação predominante entre as membranas é puramente de natureza eletrostática ou puramente de natureza entrópica devido às flutuações. Nenhum desses modelos é adequado para descrever os resultados obtidos neste trabalho, provavelmente porque para as fases lamelares estudadas há uma combinação dessas interações. A interação eletrostática no caso da membrana de lecitina seria possivelmente devido à interações dipolares entre os grupos fosfato em interfaces opostas ou ainda entre moléculas de água organizadas em torno desses grupos.

Combinando os resultados dos experimentos de SAXS com os experimentos à umidade relativa controlada foi possível determinar as constantes que caracterizam as propriedades elásticas da membrana; o módulo de compressibilidade e a constante de rigidez. A ordem de grandeza dos valores obtidos para a membrana de lecitina é compatível com valores disponíveis na literatura, porém mostram que rigidez da membrana varia com a hidratação.

A inserção de moléculas de Simulsol na membrana, no regime confinado, resulta no aumento da constante de rigidez, que pode ser explicado pela superposição das cadeias poliméricas das moléculas de Simulsol, porém no regime diluído, contribuem para o aumento da flexibilidade da membrana, reduzindo drasticamente a constante de rigidez.

Barbara Bianca Gerbelli - Propriedades estruturais e elásticas de fases lamelares: O efeito da composição da membrana. 


\subsection{Perspectivas}

Estão programados novos experimentos para determinar com maior precisão as fronteiras do domínio bifásico e a existência de um limite de diluição para as membranas mais ricas em Simulsol. Para descrever de maneira mais precisa o efeito da incorporação do Simulsol à membrana devem ser realizados experimentos de GISAXS para outras composições de membranas. Esses resultados serão utilizados para a redação de artigos científicos.

Os resultados obtidos neste trabalho fornecem subsídios para preparação de complexos de lipídios não catiônicos com DNA. A composição da membrana desempenha um papel fundamental no controle das interações de origem entrópicas e na estabilização da fase lamelar. É possível, para uma dada periodicidade lamelar, preparar membranas com propriedades elásticas distintas a partir da escolha da sua composição. Por outro lado, também foi mostrado que é possível preparar fases lamelares com espessuras de camada aquosa de cerca de $100 \AA$ Å, abrindo-se a possibilidade de encapsulação de fragmentos de DNA mais longos. Partindo primeiramente de tamanhos muito pequenos (menores de $150 \mathrm{pb}$ ), que se comportam como esferas rígidas podemos passar para fragmentos de $150 \mathrm{pb}$ que se comportam como bastões rígidos, e aumentando ainda mais o número de pares de base, os fragmentos devem se comportar como cadeia flexível.

Barbara Bianca Gerbelli - Propriedades estruturais e elásticas de fases lamelares: O efeito da composição da membrana. 



\section{A.1. Adaptações mecânicas para a instalação da técnica de GISAXS}

A primeira adaptação feita foi por meio de uma placa que é colocada entre a base do porta amostra fixo na câmara responsável pelo movimento do porta amostra nas coordenadas de X e Y e o goniômetro. As furações e a fixação na base do motor são mostradas na figura 2, com 2 parafusos M4 nas posições indicadas pelas setas. O goniômetro é fixado

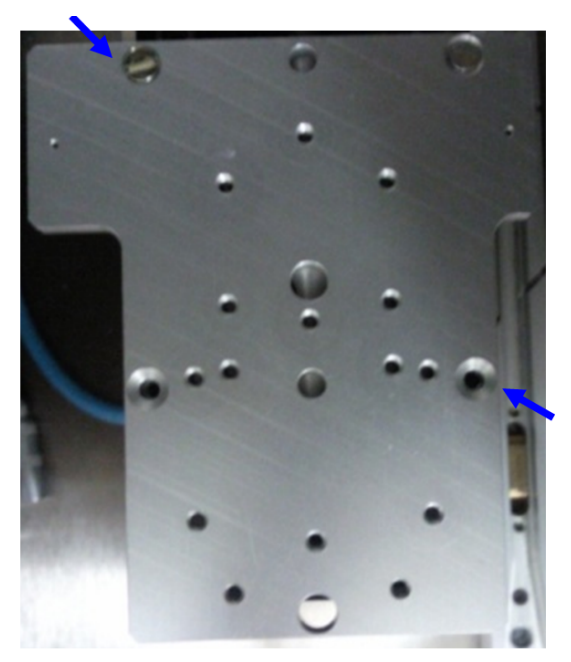

Figura A.r.: Adaptador que encaixa na base do porta amostra e no goniômetro

então na placa por meio de 2 parafusos mostrados com setas na figura A.2. Na figura A.2(b) pode-se ver o goniômetro já montado sobre a base.

Barbara Bianca Gerbelli - Propriedades estruturais e elásticas de fases lamelares: O efeito da composição da membrana. 


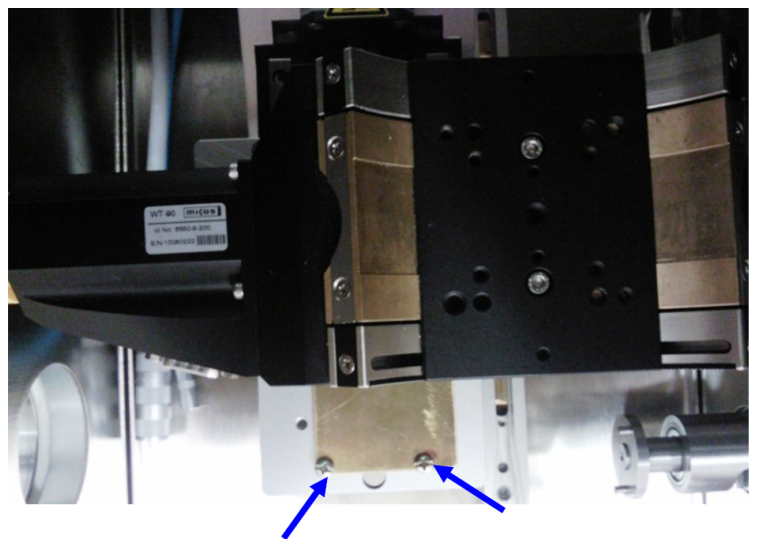

(a)

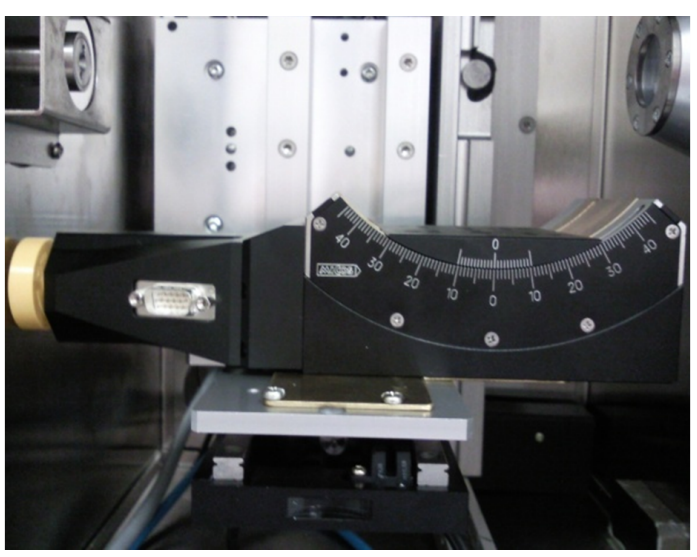

(b)

Figura A.2.: (a) Vista de cima e (b) lateral da base de adaptação entre o goniômetro e a base de adaptação da base do porta amostra.

Na parte superior do goniômetro foi instalada uma mesa ótica que permite um pequeno movimento na direção perpendicular ao movimento do goniômetro (Figura A.2(b) ). Essa mesa possui ajustes finos para a rotação da amostra e ajuste do ângulo azimutal (Figura A.3(a)e A.3(b)).

A regulagem do ângulo de Bragg (20) é feita por meio de rotações em torno do eixo $\mathrm{z}$, que são feitas no goniômetro. As rotações em torno dos eixos x e y permitem, regulagens dos ângulos $\Psi$ e $\varphi$, utilizando os parafusos da mesa ótica.

Barbara Bianca Gerbelli - Propriedades estruturais e elásticas de fases lamelares: O efeito da composição da membrana. 


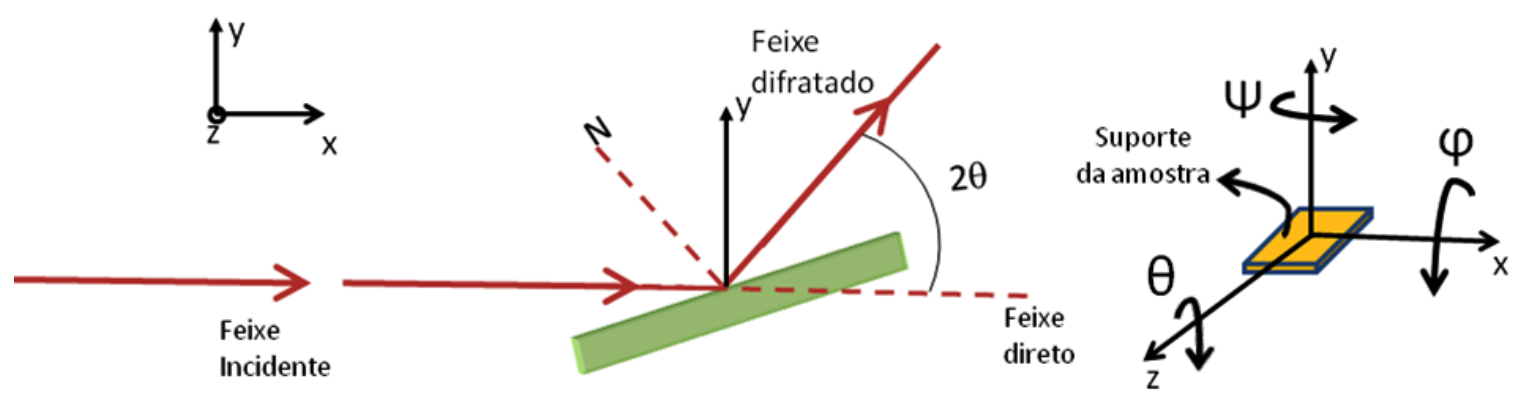

(a)

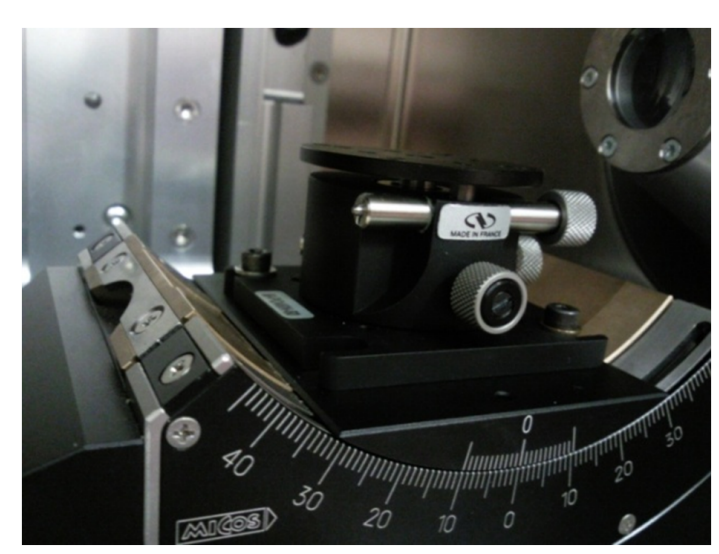

(b)

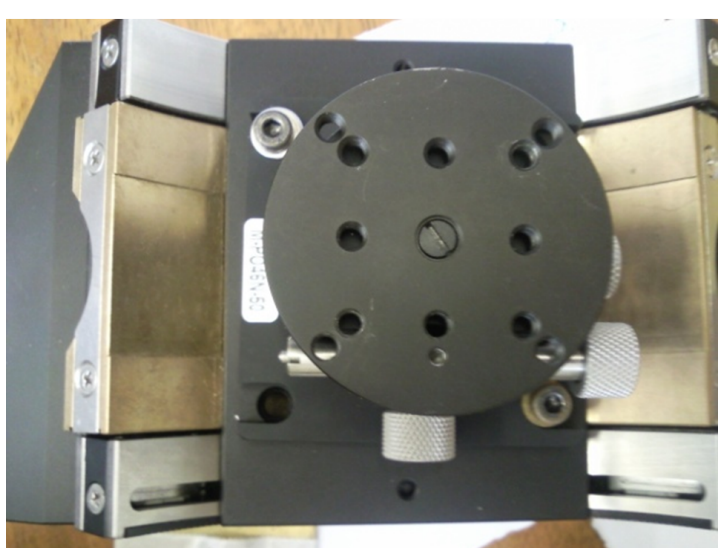

(c)

Figura A.3.: (a) Esquema da montagem experimental e descreve os possíveis movimentos em relação a amostra. (c) vista Lateral e (d) superior da mesa ótica instalada em cima do goniômetro.

Barbara Bianca Gerbelli - Propriedades estruturais e elásticas de fases lamelares: O efeito da composição da membrana. 



\section{B.1. Potencial químico da água na fase lamelar}

Para descrever o comportamento termodinâmico de uma fase lamelar parte-se da expressão do potencial de interação entre as bicamadas, que tem a contribuição de um potencial atrativo $\left(\boldsymbol{V}_{\boldsymbol{a}}\right)$ de van der Waals, de longo alcance, e um potencial repulsivo de curto alcance $\left(\boldsymbol{V}_{\boldsymbol{R}}\right)$. Para expressar o potencial repulsivo, utilizaremos uma expressão que assume uma forma analítica aproximada, para simplificação do modelo. Assim a energia de interação entre as membranas, por unidade de superfície pode ser escrita como:

$$
V_{\text {int }}(D)=\frac{V_{R}}{\left(D-\delta_{M}\right)^{8}}-\frac{V_{a}}{\left(D-\delta_{M}\right)^{2}}
$$

onde $\boldsymbol{V}_{\boldsymbol{A}}$ e $\boldsymbol{V}_{\boldsymbol{R}}$ são constantes que podem ser estimadas a partir de dados na literatura.

A energia livre de Helmholtz será escrita em função da fração volumétrica de lipídio $\left(\phi_{l i p}\right)$, usando a relação $D=\delta_{M} / \phi_{l i p}$;

$$
\begin{gathered}
f(\phi)=\frac{\phi}{\delta_{M}}\left[\frac{V_{R}}{\left(\delta_{m}\left(\phi_{l i p}-\delta_{M}\right)^{8}\right.}-\frac{V_{a}}{\left(\delta_{m}\left(\phi_{l i p}-\delta_{M}\right)^{2}\right.}\right] \\
f(\phi)=\frac{\phi_{l i p}}{\delta_{M}}\left[\frac{V_{R}}{\delta_{M}^{8}} \frac{\phi_{l i p}^{8}}{\left(1-\phi_{l i p}\right)^{8}}-\frac{V_{a}}{\delta_{M}^{2}} \frac{\phi_{l i p}^{2}}{\left(1-\phi_{l i p}\right)^{2}}\right] .
\end{gathered}
$$

Barbara Bianca Gerbelli - Propriedades estruturais e elásticas de fases lamelares: O efeito da composição da membrana. 


$$
\begin{gathered}
f(\phi)=\frac{\phi_{l i p}^{3}}{\delta_{M}^{9}}\left[V_{R}\left(\frac{\phi_{l i p}^{6}}{\left(1-\phi_{l i p}\right)^{2}}\right)-V_{a t r}\left(\frac{\phi_{l i p}^{6}}{\left(1-\phi_{l i p}\right)^{2}}\right)\right] \\
f(\phi)=\frac{V_{R} \phi_{l i p}^{3}}{\delta_{M}^{9}}\left[\frac{\phi_{l i p}^{6}}{\left(1-\phi_{l i p}\right)^{8}}-\frac{V_{a t r} \phi_{l i p}^{6}}{V_{R}} \frac{1}{\left(1-\phi_{l i p}\right)^{2}}\right] \\
f(\phi)=\bar{F} \phi_{l i p}^{3}\left[\frac{\phi_{l i p}^{6}}{\left(1-\phi_{l i p}\right)^{8}}-\frac{C}{\left(1-\phi_{l i p}\right)^{2}}\right]
\end{gathered}
$$

Na equação acima a $\overline{\boldsymbol{F}}$ e C são constantes dadas por: $\overline{\boldsymbol{F}}=\boldsymbol{V}_{\boldsymbol{R}} / \boldsymbol{\delta}^{\mathbf{9}}, \boldsymbol{C}=\boldsymbol{V}_{\boldsymbol{A}} \cdot \boldsymbol{\delta}^{\mathbf{6}} / \boldsymbol{V}_{\boldsymbol{R}}$ para a região de limite de diluição. O potencial químico do solvente pode então ser calculado;

$$
\mu_{s}=\frac{\partial F}{\partial N_{w}}
$$

onde $N_{s}$ refere-se ao número de moléculas do solvente, e $\boldsymbol{F}=\boldsymbol{V} \boldsymbol{f}\left(\phi_{\text {lip }}\right)$ e V é o volume total da fase lamelar. Por sua vez, o volume da fase lamelar pode ser expresso em termos do volume molecular do solvente $\left(\boldsymbol{v}_{\boldsymbol{w}}\right)$ do volume molecular do soluto, que nesse caso é a molécula de lipídio $\left(\boldsymbol{v}_{l i p}\right)$;

$$
\boldsymbol{V}=\left(\boldsymbol{N}_{\boldsymbol{w}} \boldsymbol{v}_{w}+\boldsymbol{N}_{\text {lip }} \boldsymbol{v}_{\text {lip }}\right)
$$

Portanto, a energia livre da fase lamelar pode ser expressa como:

$$
F=\left(N_{w} v_{w}+N_{l i p} v_{l i p}\right) f\left(\phi_{l i p}\right)
$$

e a fração volumétrica de lipídios será calculada por:

$$
\phi=\frac{N_{l i p} v_{l i p}}{N_{w} v_{w}+N_{l i p} v_{l i p}}
$$

Derivando a expressão (B.6) em relação a $\boldsymbol{N}_{\boldsymbol{w}}$, obtém-se o potencial químico do solvente, ou seja da água;

$$
\mu_{s}=\frac{\partial F}{\partial N_{w}}=v_{w} f(\phi)+\left(N_{w} v_{w}+N_{l i p} v_{l i p}\right)\left[\frac{N_{w} v_{w}}{\left(N_{w} v_{w}+N_{l i p} v_{l i p}\right)^{2}}\right]
$$

Barbara Bianca Gerbelli - Propriedades estruturais e elásticas de fases lamelares: O efeito da composição da membrana. 


$$
\mu_{s}=v_{w}\left[f\left(\phi_{l i p}\right)-\phi_{l i p} \frac{\partial f}{\partial \phi_{l i p}}\right]
$$

Derivando a expressão B. 3 com relação a $\phi$, tem-se:

$$
\frac{\partial f}{\partial \phi_{l i p}}=\frac{9 \phi_{l i p}^{8}}{\left(1-\phi_{l i p}\right)^{8}}+\frac{8 \phi_{l i p}^{9}}{\left(1-\phi_{l i p}\right)^{9}}-\frac{3 C \phi_{l i p}^{2}}{(1-\phi)_{l i p}^{2}}-\frac{3 C \phi_{l i p}^{3}}{\left(1-\phi_{l i p}\right)^{3}}
$$

rearranjando os termos:

$$
\begin{gathered}
\frac{\partial f}{\partial \phi}=\frac{9 \phi_{l i p}^{8}\left(1-\phi_{l i p}\right)+8 \phi_{l i p}^{9}}{\left(1-\phi_{l i p}\right)^{9}}-\frac{C}{\left(1-\phi_{l i p}\right)^{3}}\left[3 \phi_{l i p}^{2}\left(1-\phi_{l i p}\right)+2 \phi_{l i p}^{3}\right] \\
-\phi \frac{\partial f}{\partial \phi_{l i p}}=\frac{9 \phi_{l i p}^{9}\left(1-\phi_{l i p}\right)+\phi_{l i p}^{10}}{\left(1-\phi_{l i p}\right)^{3}}+\frac{C}{\left(1-\phi_{l i p}\right)^{3}}\left[3 \phi_{l i p}^{3}-\phi_{l i p}^{4}\right] \\
f\left(\phi_{l i p}\right)=\frac{\phi_{l i p}^{9}\left(1-\phi_{l i p}\right)}{\left(1-\phi_{l i p}\right)^{9}}-\frac{C \phi_{l i p}^{3}\left(1-\phi_{l i p}\right)}{\left(1-\phi_{l i p}\right)^{3}}
\end{gathered}
$$

Substituindo $f\left(\phi_{l i p}\right)$ e $\boldsymbol{\partial f} / \partial \phi_{\text {lip }}$ na expressão B.9, obtém-se finalmente o potencial químico da água na fase lamelar, dado por:

$$
\mu_{s}=\frac{-8 \phi_{l i p}^{9}}{\left(1+\phi_{l i p}\right)^{9}}+\frac{C 2 \phi_{l i p}^{3}}{\left(1-\phi_{l i p}\right)^{9}}
$$

Onde a constante $\mathrm{C}$ foi estimada e é aproximadamente igual a 1, 92.

Barbara Bianca Gerbelli - Propriedades estruturais e elásticas de fases lamelares: O efeito da composição da membrana. 



\section{APÊNDICE C}

\section{C.1. Modelo de bicamada rígida}

A fase lamelar pode ser descrita como um empilhamento regular de bicamadas de espessura $\boldsymbol{\delta}_{M}$ separadas por uma camada aquosa de espessura $\boldsymbol{\delta}_{\boldsymbol{w}}$. A periodicidade lamelar é determinada pelos experimentos de espalhamento de raios $\mathrm{X}$, dado por $\boldsymbol{D}=\mathbf{2 \pi} / \boldsymbol{q}_{1}$, onde $\boldsymbol{q}_{1}$, é o vetor de onda do pico de primeira ordem.

Considerando um certo volume ocupado pela fase lamelar, em uma caixa de comprimento H, e área transversal A (figura C. I), o número de bicamadas $\left(\boldsymbol{n}_{\boldsymbol{b}}\right)$ na caixa, é igual a $\boldsymbol{n}_{\boldsymbol{b}}=\boldsymbol{H} / \boldsymbol{D}$.

$\mathrm{O}$ volume da caixa pode ser expresso em termos do volume molar do solvente e do soluto (lipídio) como:

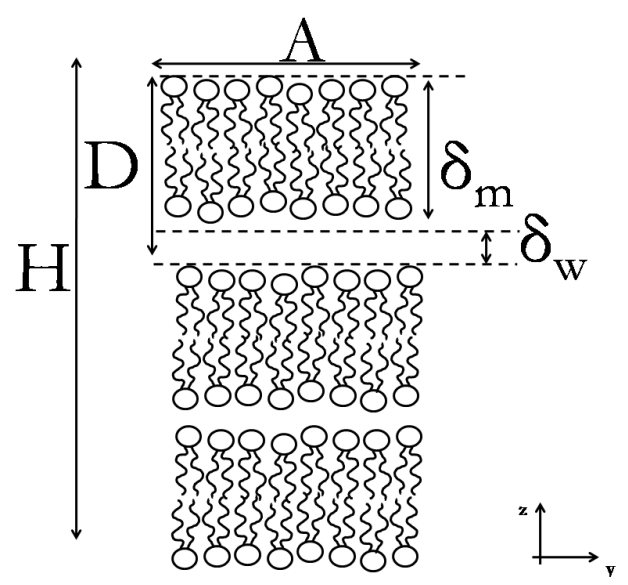

Figura C. .: Desenho esquemático representando a célula unitária de uma fase lamelar.

Barbara Bianca Gerbelli - Propriedades estruturais e elásticas de fases lamelares: O efeito da composição da membrana. 


$$
V=N_{w} \cdot v_{w}+N_{l} v_{l}=H . A .
$$

Onde os índices $s$ e $l$ referem-se ao solvente (água) e lipídio respectivamente, e $\mathrm{N}$ é o número de moléculas de cada espécie.

A razão entre o volume total ocupado pelas moléculas de lipídios e o volume da caixa, é a fração volumétrica de lipídios;

$$
\phi_{w}=\frac{N_{w} v_{w}}{H A}=\frac{n_{b} \delta_{M} A}{H A},
$$

ou ainda,

$$
D=\delta_{M} / \phi_{l i p}
$$

A equação acima, chamada de lei de diluição. Em um gráfico de $\mathrm{D}$ em função de $1 / \phi_{l i p}$, os pontos experimentais devem estar sobre uma reta, cujo coeficiente angular é a espessura da membrana.

Outro parâmetro importante para caracterizar o empacotamento das moléculas anfifílicas é área por cabeça polar na superfície da bicamada, $\sum$. Para uma dada área transversal A, da bicamada, pode-se escrever que:

$$
A=\frac{N_{l} \sum}{2 n_{b}} .
$$

Tomando $\mathrm{H}=\mathrm{D}$, na eq. C.2, e substituindo o valor de A, tem-se:

$$
\phi=\frac{2 v_{l i p}}{\sum D} .
$$

Combinando a expressão acima com a lei de diluição, pode-se ainda escrever que:

$$
\delta_{m}=\frac{2 v_{l i p}}{\sum},
$$

Barbara Bianca Gerbelli - Propriedades estruturais e elásticas de fases lamelares: O efeito da composição da membrana. 
Esse modelo geométrico foi utilizado como argumento para prever, que o aumento na espessura da bicamada está relacionado á diminuição da área ocupada por surfactante, considerando o volume molecular constante.

Barbara Bianca Gerbelli - Propriedades estruturais e elásticas de fases lamelares: O efeito da composição da membrana. 



\section{D.1. Artigo publicado no Journal of Applied Crystallography (J. Appl. Cryst. (2012). 45, 1278-1286)}

Gaussian Deconvolution: A powerful method for form-free modeling scattering data from mono and multilayered planar systems using constrained least-square fitting

Cristiano L. P. Oliveira, Barbara B. Gerbelli, Emerson R. T. Silva, Frédéric Nallet, Laurence Navailles, Elisabeth A. Oliveira and Jan Skov Pedersen

\section{Abstract}

A new method for analysis of scattering data from lamellar bilayer systems is presented. The method employs a form-free description of the cross-section structure of bilayer and the fit is performed directly to the scattering data introducing also a structure factor when required. The cross-section structure (elec-tron density profile in the case of x-ray scattering) is described by a set of Gaussian functions and it is termed Gaussian deconvolution. The coefficients of the Gaussians are optimized using a constrained least-squares routine that induces smoothness on the electron density profile. The optimization is coupled with the point-of-inflection method (Glatter, 1977) for determining the optimal weight of the smoothness. With the new approach, it is possible to optimize simultaneously the form factor, structure factor and several other parameters in the model. The applicability of this method is demonstrated by using it in a study of a multilamellar system composed of lecithin bilayers, where the form factor and structure factor are obtained

Barbara Bianca Gerbelli - Propriedades estruturais e elásticas de fases lamelares: O efeito da composição da membrana. 
simultaneously and the obtained results provided new insight into this very well-known system.

\section{Introduction}

Lamellar systems of lipid bilayers have been widely used as model system for biological membranes. They allow the study of, for example, phase transitions, and interactions between membranes and with biological macromolecules. The organization of the lipid molecules on the microscopic level is closely related to macroscopic properties of membrane, and determines the key properties such as elasticity, permeability to small molecules and interactions with proteins or peptides. X-ray scattering techniques are particularly suited for obtaining information of the microscopic organization of the molecules. Since the pioneering work of Luzzati (Luzzati et Tardieu, 1974), many researchers have developed new methods to analyze experimental data allowing determination of relevant parameters characterizing the lamellar structure.

Several methods for analyzing X-ray scattering profiles for lamellar systems have been proposed. Seminal works using Gaussians functions to describe the electron density profile were described by Pape and Kreutz (Pape et Kreutz, 1978) and by Nagle and Wiener (Nagle et Wiener, 1989), however, these me-thods are very sensitive to the first estimate of the parameters describing the profile, i.e., they require good a priori information about the signs and the positions of the Gaussians. The situation is even more complicated when multilayered lamellar systems have to be described and several strategies for modelling the form factor and structure factor effects have been proposed (Nallet et al., 1993; Nagle et al., 1996; Petrache et al., 1998). More recently, an approach was presented by Pabst (Pabst et al., 2000), where Gaussian functions were again used to describe the lamellar cross-section profile, and as the coupling between the form factor and structure factor was taken into account, the full scattering intensity profile could be described by this method. However, the approach was restricted to the use of only two Gaussian functions to avoid model instabilities as previously mentioned by other authors (Pape et Kreutz, 1978).

An approach to overcome instabilities in the modeling of electron density profiles was introduced in the so-called Square-Root Deconvolution Method, originally proposed by Glatter (Glatter, 1981). It enables retrieving of the electronic density profile for a given geometry (planar, cylindrical or spherical) by describing the electron density profile by a set of basis functions (step functions) and using these for modeling the pair distance distribution function. The method was originally applied to scattering data from uncorrelated objects (i.e., without structure factor effects) but later was also adapted for including possible high order arrangements (Fritz et Glatter, 2006). However, one of the limitations

Barbara Bianca Gerbelli - Propriedades estruturais e elásticas de fases lamelares: O efeito da composição da membrana. 
of this approach is the need of making the intermediate step of first determining the pair distance distribution function which might preclude the optimization of other structural features that might also be present, such as, for example, free bilayers (Pabst et al., 2000). On the other hand, the main advantage of this procedure is the possibility of retrieving the electron density profile on a free form without any previous assumptions. The number of degrees of freedom in the expression for the electron density profiles is usually large and this means that the solution has to be 'stabilized'. This stabilization can be done by the addition of a smoothness constraint to the usual chi-squared as a penalty function with a certain weight relative to chi-squared. The weight of the penalty function is determined by the use of point-of-inflection method during the minimization procedure (Glatter, 1977). A similar two-step analysis method for analysis of specular reflectivity data was introduced by Pedersen (Pedersen, 1992). A new and more sophisticated approach is to model the lipid membrane based on scattering density profile (SDP) methods (Heberle et al., 2012). This approach permits the inclusion of molecular information and the use of molecular dynamics simulations as inputs for the fitting of scattering data. Although this method can provide more advanced results, it requires several a priori information about the system, its composition and fine details about the lipid molecules. As will be described below, the Gaussian deconvolution approach, on the other hand, does not require any a priori information for the fitting procedure and the obtained profile can be used as input for other more sophisticated analysis.

In the present work a new method is presented for modelling Small-Angle X-ray Scattering experimental data for systems containing both unilamellar and multilamellar planar membranes. As will be discussed in next section, a model approach similar to the one used by Glatter in his deconvolution method (Glatter, 1981) can be used to stabilize the modeling of the electron density profile by a set of Gaussian functions. However, the main advantage of this approach is the fact that the scattering intensity is adjusted and not the pair distance distribution function. This is similar to the method introduced by Pedersen and Hamley (Pedersen et Hamley, 1994a, b) for analysis of specular reflectivity data. By the new method it is possible to retrieve a smooth electron density profile for which the calculated intensity gives the best fit of the experimental data. The approach allows, by the use of the least-squares procedure, the simultaneous determination of the amplitudes of the Gaussian functions that describe the electron density profile and several other parameters as, for example, the ones describing structure factor effects, backgrounds, etc.

The new method is applied for determining the swelling properties of a lamellar phase composed of lecithin bilayers, providing a good fit quality for the full set of experimental

Barbara Bianca Gerbelli - Propriedades estruturais e elásticas de fases lamelares: O efeito da composição da membrana. 
scattering curves for a series of increasing degree of membrane hydration. New insight emerges from the analysis of the experimental data on the structure of the bilayer as extracted from the electronic contrast profile. It is shown that the Caille parameter, which characterizes the flexibility of lipid membranes together with inter-membrane interactions, passes through two regimes. This provides information on the role of hydration and fluctuation forces for stabilizing the lamellar structure.

Theoretical Model description The scattering from a system of scattering objects can be described by the following expression:

$$
I(q)=c<P(q)_{3 D} S(q)>
$$

where $c$ is related to the concentration of particles in the system, $P(q)_{3 D}=|\boldsymbol{F}(q)|^{2}$ is the form factor of the three dimensional objects and carries information about particles shapes and contrasts and $\mathrm{S}(\mathrm{q})$ is the system structure factor, which carries information about possible interparticle interactions and ordering. The parameter $\mathrm{q}$ is the modulus of the scattering vector and it is given by $q=4 \pi \sin (\theta) / \lambda$, where $2 \theta$ is the scattering angle and $\boldsymbol{\lambda}$ is the wavelength of the radiation. The brackets " $\langle>$ " indicate that in the general case these two contributions have to be averaged together in the calculation. For locally highly anisotropic systems, like planar systems or large vesicles for example, where the transverse dimensions are much larger than the perpendicular dimension, the form factor can be described by a product of a form factor, for the perpendicular direction (ID function), and a factor $1 / q^{2}$ related to the transverse dimensions. With this, equation ?? can be rewritten as (Glatter and Kratky, i 982),

$$
I(q)=\frac{c}{q^{2}}<P(q) S(q)>
$$

An often used approach is to use the decoupling approximation and treat the form factor and structure factor separately (Kotlarchyk and Chen, i 983). For a multilayered lamellar system, with periodicity $d$, the structure factor is well described by the Modified Caillé Theory (MCT) (Zhang et al., i 994);

$$
S_{0}(q)=1+2 \sum_{k=1}^{N-1}\left(1-\frac{n}{N}-k\right) \cos (k q D) \exp \left[-\left(\frac{D}{2 \pi}\right)^{2} q^{2} \eta \gamma\right](n \pi)^{-\left(\frac{D}{2 \pi}\right) q^{2} \eta(\mathrm{D} .3)}
$$

The mean number of coherent scattering bilayers in the stack is denoted as $\mathrm{N}$, and $\gamma$

Barbara Bianca Gerbelli - Propriedades estruturais e elásticas de fases lamelares: O efeito da composição da membrana. 
is Euler's constant. The Caillé parameter $\boldsymbol{\eta}$ is related to both the bending modulus $\mathrm{K}$ of lipid bilayers and the bulk modulus $\bar{B}$ for compression (Zhang et al., I994; Caillé, 1972);

$$
\eta=\frac{q_{0}^{2} k_{B} T}{8 \pi \sqrt{K \bar{B}}}
$$

In this expression $\mathrm{k}$ is Boltzmann's constant and $\mathrm{T}$ is the absolute temperature. The description of the form factor can be done at several levels of detail. The form factor has to describe the differences in electron density for the molecules constituting the bilayer. In addition, for several applications it is a good approximation to assume that the bilayer is symmetric with respect to its centre. One of the simplest ap-proximations is to consider a two-steps model given by (Worthington, 1969; Nallet et al., 1993; Brotons et al., 2005),

$$
P(q)=\frac{4 \Delta \rho_{H}^{2}}{q^{2}}\left\{\sin \left(q\left(\delta_{H}+\delta_{T}\right)\right)-\sin \left(q \delta_{T}\right)+\frac{\Delta \rho_{T}}{\Delta \rho_{H}} \sin \left(q \delta_{T}\right)\right\}^{2} .
$$

where $\Delta \rho_{H}$ and $\Delta \rho_{T}$ are, respectively, the head group and tail group electron density contrasts and $\delta_{H}$ and $\delta_{T}$ are the respective sizes of the head group and tail group regions. Since this expression is very simple, it usually gives a too crude description of the electron density profile and in several cases a better descrip-tion is needed. With a similar step-model strategy, Glatter and co-workers developed the deconvolution square-root method (Glatter, I98 I; Fritz and Glatter, 2006) where the electron density is described by a series of step functions. The height of the steps are determined using a constrained least-squares fitting procedure. However, in this approach the fit is done to the pair distance distribution and not directly to the experimental intensity. The intermediate step of determining the pair distance distribution function by a Fourier transform can cause several limitations for the method.

Another strategy was proposed by Pabst et al. (Pabst et al., 20oob), who suggested to model the bilayer using two Gaussian functions: A central Gaussian placed at the origin, which models the central part of the bilayer where hydrocarbon tails from the opposite sides meet and a second Gaussian placed at a certain distance for modeling the head group region. The central part is known to have negative con-trast with respect to a water buffer for X-Rays, whereas the headgroups are electron rich and have a posi-tive contrast. This model is also a crude approximation of the electron density profile since it assumes that the tail groups have almost the same electron density as the water and that it is only the most central part of the bilayers where the hydrocarbon chain meets that have a negative

Barbara Bianca Gerbelli - Propriedades estruturais e elásticas de fases lamelares: O efeito da composição da membrana. 
contrast. But surprisingly, the model is sufficient to obtain a reasonable description of the scattering data for several examples in the literature (Pabst et al., 200oa). The smoothness introduced by the Gaussian profiles seems to improve the fit quality as compared to the simple two step model. However, this model also fails for high quality data, in particular for high q values where high-resolution information on the structure is probed. In the present work we combine the advantages of the Gaussian description of the electron density profiles with the stability introduced by Glatter in the free-form method. This enables a more flexible and stable modeling procedure. In the new approach, the profile is described by a set of equally spaced Gaussian functions. During the optimization, it is necessary to constrain only the amplitudes of the Gaussian functions and not the other model parameters. As will be shown in the next section, this can be done by a proper change of the design matrix of the least-squares problem, introducing the Lagrange multiplier $\boldsymbol{\lambda}$ only at the necessary positions in the matrix. During the fitting procedure, the Lagrange multiplier is varied and the other parameters are optimized for each $\boldsymbol{\lambda}$ value. By using the point-of-inflection method, it is possible to obtain the $\lambda$ value by an automatic procedure that gives both a good fit to the scattering data and the optimum constraint of the Gaussians amplitudes. With this approach is possible to obtain a smooth profile that can describe more accurately the electron density of the lipid bilayer simultaneously with the optimization of the structure factor and other model parameters. In the following we present the procedure for symmetric and asymmetric bilayers.

\section{Symmetric Bilayer}

For the description of a symmetric planar bilayer, a given number, $\boldsymbol{n}_{\boldsymbol{m a x}}$, of Gaussian functions are used for describing the profile. This basis of functions is constructed in such a way that there is a central Gaussian at $z=\mathbf{0}$ and that the rest of the Gaussians are at equidistant positions mirrored so that a symmetric profile is obtained. The central Gaussian can represent the ends of the bilayer tail groups and the other centro-symmetric Gaussians build the remaining parts corresponding to the tail groups and the head groups. The electron density is thus described by:

$$
\begin{array}{r}
\rho(z)=\sum_{n=1}^{4} \frac{a_{n}\left[G_{s}\left(z, z_{n}, \sigma_{n}\right)+G_{s}\left(z-z_{n}, \sigma_{n}\right)\right]}{1+\delta_{i j}} \\
\delta_{i 1}=1, i=1 \text { e } \delta_{i 1}=0, i=2,3,4
\end{array}
$$

Barbara Bianca Gerbelli - Propriedades estruturais e elásticas de fases lamelares: O efeito da composição da membrana. 


$$
G_{s}\left(z, z_{n}\right), \sigma_{n}=\frac{1}{\sigma \sqrt{2 \pi}} \exp \left\{\frac{-\left(z-z_{n}\right)^{2}}{2 \sigma^{2}}\right\}
$$

and the Kronecker delta function $\delta_{i 1}$ is used to avoid double counting for the central Gaussian. $\mathrm{n}$ is the order of Gaussian used. The profile is defined by the values of the amplitudes an. The half bilayer width $\mathrm{Z}$ is defined as the distance between the center of the bilayer and the posi-tion where the last Gaussian reaches a width of two standard deviations $(\sigma)$. With this definition, the positions of the Gaussians and the standard deviations ( $\boldsymbol{\sigma}$ values) are calculated as follows:

$$
z_{n}=(n-1) 2 \sigma, \sigma=\frac{Z}{2 n_{\max } \sqrt{2 \ln 2}}
$$

This choice of the centers and standard deviation gives a reasonable overlap between the Gaussian func-tions enabling the construction of smooth profiles. The choice of the number of Gaussian functions (nmax) will depend on the level of details that is needed to build the profile. Since the scattering profile contains a low information content (Moore, 1980) a general rule that can be used is to use the minimum required number of terms for the modeling, which can be obtained from a few simple tests. If it is used a number of terms larger than the ideal value, the obtained profile will have the same shape but some artificial oscillations. A typical profile built using equation ?? is shown in Figure IA. One of the advantages of using a Gaussian set of functions for the representation of the profile is that, for the one-dimensional case of centro-symmetric bilayers, the Fourier transform of the electron density has an analytical solution, providing the scattering amplitudes (Abramowitz and Stegun, 1972; Pabst et al., 2000a):

$$
F(n, q)=\sqrt{2 \pi \sigma} \exp \left(-\frac{\sigma^{2} q^{2}}{2}\right) \cos \left(q z_{n}\right)
$$

The final profile scattering amplitude of the lamellar cross section is just the sum of all the $\mathrm{F}(\mathrm{q}, \mathrm{n})$ terms:

$$
F(q)=\sum_{n=1}^{n_{m} a x} a_{n} F(q, n)
$$

Barbara Bianca Gerbelli - Propriedades estruturais e elásticas de fases lamelares: O efeito da composição da membrana. 
and the form factor of the infinite lamellar system is:

$$
P(q)=|F(q)|^{2}
$$

A theoretical simulation is shown in Figure ${ }_{\mathrm{I}} \mathrm{B}$, where a profile with four Gaussian functions was used and combined with the structure factor of the Modified Caille theory to provide the combined I(q) intensity.

\section{Asymmetric Bilayer}

An expression for the form factor from the asymmetric bilayer was proposed by Brzustowicz and

$$
\begin{array}{rr}
P(q)= & \text { (D. I 5) } \\
<F(q)^{2}>=\sum_{k, k_{1}} a_{k} a_{k_{1}} \sigma_{k} \sigma_{k_{1}} \exp \left[-q^{2} \sigma_{k}+\sigma_{k_{1}} / 2\right] \cos \left[q\left(z_{k}+z_{k_{1}}\right)\right] &
\end{array}
$$

where $\sigma_{i}$ are the Gaussians widths, ai are the Gaussian amplitudes and zi are the Gaussians centers, with respect to a common origin. For this case the construction of the profile is slightly different. Since the symmetry is lost, the electron density profile is given by

$$
\rho(z)=\sum_{n=1}^{n} a_{n} G_{s}\left(z, z_{n}, \sigma\right)
$$

In this case, $\mathrm{Z}$, which is a measure of the full bilayer thickness, the centers of the Gaussians, and the stan-dard deviation ( $\boldsymbol{\sigma}$ value) are defined and related as follows:

$$
z_{n}=(n-1) 2 \sigma, \sigma=\frac{Z}{2 n_{\max } \sqrt{2 \ln 2}}
$$

This choice of the centers and standard deviation gives again a reasonable overlap between the Gaussian functions enabling the construction of smooth profiles. A typical profile made using equation D. I6 and the corresponding scattering intensity calculated by equation D. Is are shown in Figure 2.

\section{Final intensity expression}

The expressions given in the previous sections permit the calculation of the scattering intensity for the proposed electron density profiles. In the case of multilamelar planar

Barbara Bianca Gerbelli - Propriedades estruturais e elásticas de fases lamelares: O efeito da composição da membrana. 


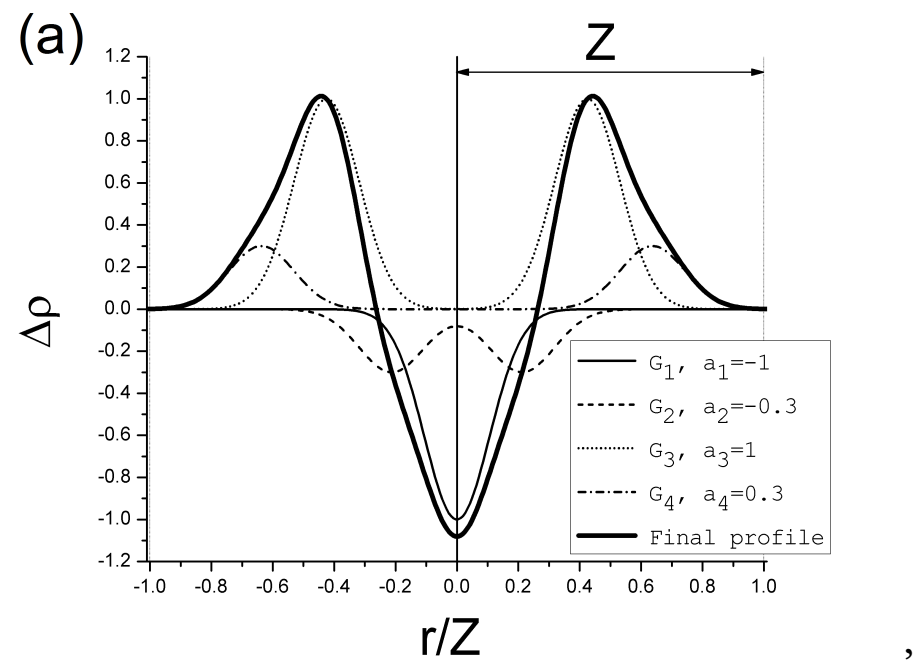

(a)

(b)

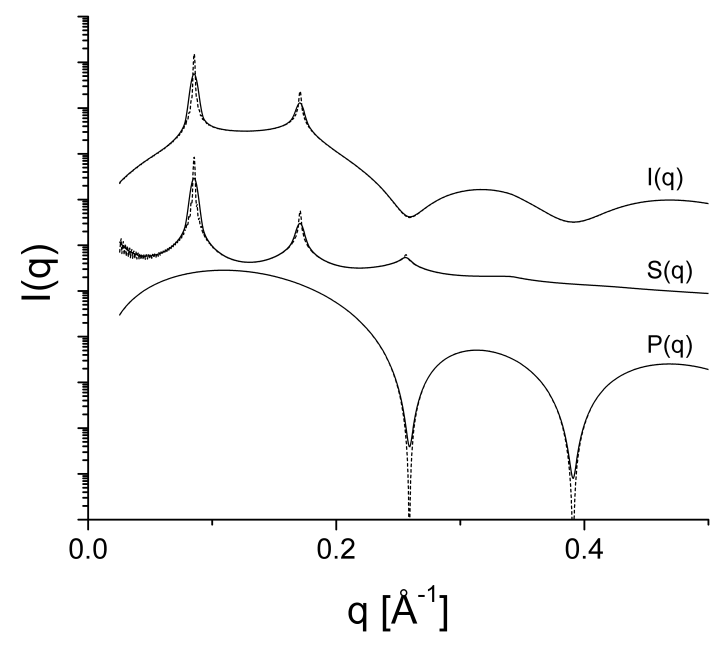

(b)

Figura D. I.: (a) Construction of the electron density profile using four Gaussians (GI, $\mathrm{G}_{2}, \mathrm{G}_{3}$ and $\left.\mathrm{G}_{4}\right)$ with four different amplitudes $(-1,-0.3,1,0.3)$. They are shown by four different line styles. The final resultant electron density profile from the Gaussian model is shown in solid thick line. (b) Final intensity and respective form factor and structure factor. In each figure the instrumentally smeared (solid line) and ideal non-smeared (dotted line) curves are presented. In this simulation the number of layers used was $N=\mathbf{5 0}$, the interplanar distance $d=73.5(0.2) \AA$, bilayer half size of $Z=30$ Åand the Caillé parameter $\boldsymbol{\eta}=\mathbf{0 . 1 2}$.

Barbara Bianca Gerbelli - Propriedades estruturais e elásticas de fases lamelares: O efeito da composição da membrana. 
(a)

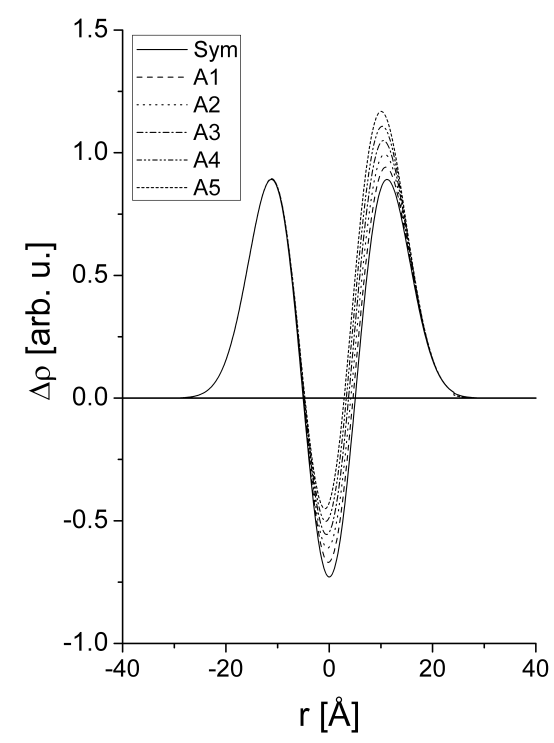

(a) (b)

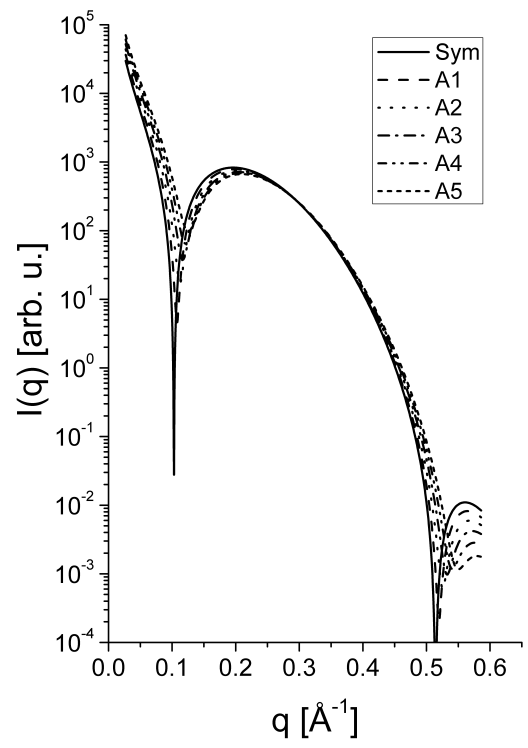

(b)

Figura D.2.: (a) An electron density profile cstructed using five Gaussians. The profile is symmetric (Sim, solid curve) and becomes increasingly more asymmetric (A I). This asymmetry is obtained by the proper choice of the Gaussians amplitudes. (b) Calculated intensity for the different profiles. Interestingly, the breaking of the symmetry induces significantly changes on the form factor profile.

membranes (or large vesicles), this electron density profile will be a repeating unit and a structure factor has to be included for describing the ordered stacks. In the present context it is the structure factor from the modified Caille theory (equation 3), which is used and the final scattering intensity of the cross-section structure of the system is then (Pabst et al., 2000a):

$$
I_{M L V}(q)=\left(|F(q)|^{2} S_{M C T}(q)+N_{d i f f}|F(q)|^{2)}\right.
$$

where the second term is included for describing the diffuse scattering which might appear from the presence of single bilayers in the system. Instrumental smearing effects, which are a consequence of the collimation of the camera pinholes, wavelength bandwidth, detector resolution, etc, can be taken into account by smearing the intensity expression by the resolution function $\boldsymbol{R}(\langle\boldsymbol{q}\rangle, \boldsymbol{q})$ as described by Pedersen and 
co workers (Pedersen et al., 1990). The final expression used to describe the data from multilamellar planar bilayers is:

$$
I_{f i t}(q)=S_{c 1} \frac{I_{M C T}(<q>)}{q^{2}}+B a c k
$$

where $\boldsymbol{S}_{\boldsymbol{c} 1}$ is an overall scale factor and Back is a background expression. All parameters are optimized during the data fitting. One of the main advantages of this approach is the fact that the scattering intensity is adjusted and not the pair distance distribution function. In this way, it is very simple to add additional contribution for the scattering like particle flexibility (which would lead to a polymer-like contribution at high angles) or a given low scattering angle contribution, and optimize all parameters in the same least-squares fit. Using the above mentioned procedure it is possible to perform a full curve fitting, retrieving simultaneously information about the form factor and structure factor.

\section{Optimization Method}

In the previous section, the expressions for the theoretical intensity $I^{\bmod }(\boldsymbol{q})$ for planar bilayers with or without structure factor effects has been given. As mentioned above, the next step is to optimize the model parameters, which is done by a least-squares procedure. The coefficients are obtained by the minimization of the weighted chi-squared (or mean deviation) $\chi^{2}$

$$
\chi^{2}=\sum_{i=1}^{M}\left[I(q)^{e x p}-I^{m o d}(q)\right]^{2} / \sigma_{i}^{2}
$$

where $\mathrm{M}$ is the number of experimental points, $I^{\exp }(q i)$ and $\sigma_{i}$ are the experimental data and the standard errors, respectively. Since the theoretical intensity is not expressed as a linear combination of the model coefficients, the minimization of (17) requires the application of a nonlinear least-squares procedure. There are several minimization methods available in the literature and in the present work we applied the MarquardtLevenberg algorithm (Pedersen, 1992, 1997; Glatter and Hainisch, 1984). The set of normal equations that need to be solved iteratively are:

Barbara Bianca Gerbelli - Propriedades estruturais e elásticas de fases lamelares: O efeito da composição da membrana. 


$$
\begin{array}{r}
\sum_{v=1}^{J} \alpha_{\mu v} \Delta \alpha_{v}=-\beta_{\mu} \\
\mu=1,2 \ldots J
\end{array}
$$

where $\mathrm{J}$ is the number of fitting parameters and $\Delta \boldsymbol{\alpha}_{\boldsymbol{v}}$ are the changes in the parameters $\boldsymbol{\alpha}_{\boldsymbol{v}}$ at each interaction. The iteration procedure is continued until the changes are zero within some specified limit. The matrix $\boldsymbol{\alpha}_{\boldsymbol{\mu}}$ and the vector $\boldsymbol{\beta}_{\boldsymbol{\mu}}$ are given as follows:

$$
\begin{array}{r}
\alpha_{\mu v}=\sum_{i=1}^{M} \frac{\partial I^{m o d}(q i)}{\partial \alpha_{\mu}} \frac{\partial I^{m o d}(q i)}{\alpha_{v}} / \sigma_{i}^{2} \\
\beta_{\mu v}=\sum_{i=1}^{M}\left[I^{m o d}(q i)-I^{m o d}(q i)\right] \frac{\partial I^{m o d}(q i)}{\partial \alpha_{\mu}} / \sigma_{i}^{2}
\end{array}
$$

As required for any nonlinear procedure, a set of starting values has to be used. For the case where only the electron density profile is modeled an initial square function where all parameters have the same value, chosen in such a way that the forward scattering value is reproduced, is good enough to ensure the convergence of the minimization procedure. For more complicated cases where both form factor and structure factor are optimized, some prior knowledge or manual adjustments are required. In all cases, due to the low information content in scattering profiles and the description of a very small fraction of the reciprocal space, the solution of the inverse scattering problem (obtain real space information from the reciprocal space data) usually leads to ill poised problems, generating solutions with large oscillations, which have no physical meaning. In order to overcome this problem, it is necessary to add model constraints, which lead to solutions that are more physically reasonable. One procedure is to add a stabilizing function $\left(\boldsymbol{N}_{c}\right)$ to $\chi_{\mathbf{2}}$.

$$
\begin{array}{r}
N_{c}=\sum_{n=1}^{P-1}\left(\alpha_{n+1}-\alpha_{n}\right)^{2} \\
\chi^{2}=\lambda N_{c}=m i n
\end{array}
$$

where $\mathrm{P}$ are the number of parameters that are wanted to be constrained $(\boldsymbol{P} \leqslant \boldsymbol{J})$. The modified normal equations are (Pedersen, I992; Pedersen and Hamley, r 994a, b)

Barbara Bianca Gerbelli - Propriedades estruturais e elásticas de fases lamelares: O efeito da composição da membrana. 


$$
\sum_{v=1}^{P}\left(\alpha_{\mu v}+\lambda K_{\mu v}\right) \Delta \alpha_{v}=-\left(\beta_{\mu}+\lambda L_{\mu}\right)
$$

The matrix $\boldsymbol{K}_{\boldsymbol{\mu} v}$ is defined as,

$$
\mathrm{K}=\left(\begin{array}{ccccccc}
1 & -1 & & & & & \\
-1 & 2 & -1 & & & 0 & \\
& -1 & 2 & -1 & & & \\
& & \cdots & \cdots & \cdots & & \\
& 0 & & & -1 & 2 & -1 \\
& & & & & -1 & 1
\end{array}\right)
$$

On the right hand of (2I) $\boldsymbol{L}_{m u}$ is given by,

$$
L_{\mu}= \begin{cases}\alpha_{i}-\alpha_{i+1} & \text { for } i=1 \\ 2 \alpha_{i}-\alpha_{i+1}-\alpha_{i-1} & \text { for } 2 \leq i \leq P-1 \\ \alpha_{i}-\alpha_{i-1} & \text { for } i=P\end{cases}
$$

Since it is in general only some of the fit parameters that describe the profile, only these parameters have to be constrained. Therefore $\boldsymbol{P}<\boldsymbol{J}$ and the sum in equation (20) will run only over the parameters that are to be constrained. For example, when fitting the form factor and structure factor, only the amplitudes related to the form factor (electron density profile) have to be constrained, whereas the parameters in the structure factor or background factors should not.

The weight of the constraint relative to $\chi^{2}$ is given by the parameter $\boldsymbol{\lambda}$ (also called Lagrange multi-plier), which can be determined by the point-of-inflection method, as described by Glatter (Glatter, 1977). In this method, the optimum lambda $\left(\boldsymbol{\lambda}_{\text {opt }}\right)$ value is obtained using graphs of the dependency of $N_{c}$, the first derivative of $N_{c}$, second derivative of $\boldsymbol{N}_{c}$, and the mean deviation as a function of $\boldsymbol{\lambda}$. The parameter $\boldsymbol{\lambda}$ is generally varied over ten or more orders of magnitude with equidistant steps on a logarithm scale. In this so-called stability plot, the value given by a position where the $\mathrm{Nc}$ is on a plateau, the first derivative is at a minimum, and the second derivative is positive, while the mean deviation is still low, is the optimum Lagrange multiplier. By a proper scaling of all these quantities, it is possible to build a functional that can be minimized in order to give the most suitable $\boldsymbol{\lambda}_{\text {opt }}$.

Barbara Bianca Gerbelli - Propriedades estruturais e elásticas de fases lamelares: O efeito da composição da membrana. 


$$
\lambda_{o p t}=\operatorname{Min}\left[\zeta_{1} \chi^{2}+\zeta_{2}\left|\left(N_{c}\right)^{\prime}\right|+\zeta_{3}\left|\left(N_{c}\right)^{\prime \prime}\right|\right]
$$

where $\zeta_{1}, \zeta_{2}$ and $\zeta_{3}$ are normalization parameters and the prime and double prime denotes first and second derivative of $\boldsymbol{N}_{C}$ against $\lambda$. The normalization is performed in such a way that the variables are restricted to the interval from 0 to 1 . This minimization can be easily performed by fitting a parabolic function to the functional and the minimum of the parabola provides the optimal value $\boldsymbol{\lambda}_{\text {opt }}$. This procedure can be implemented to give an automatic determination of $\boldsymbol{\lambda}_{\text {opt }}$. It has previously been successfully applied for indirect Fourier transformation of small-angle scattering from particulate systems (Oliveira et al., 2009), providing a reliable determination of $\boldsymbol{\lambda}_{\text {opt }} \mathrm{t}$. Automatic determinations of the Lagrange multiplier in indirect Fourier transformation procedures had already been proposed by other authors (Semenyuk and Svergun, I 99i; Hansen, 2000).

The constrained optimization method and the automated point-of-inflection procedure for deter-mining the optimum Lagrange multiplier $\boldsymbol{\lambda}$ were implemented for the Gaussian deconvolution method permitting the determination of the cross-section electron density profile through the simultaneous fitting of form factor and structure factor in systems of planar multilayers. An example of the automatic determination of the Lagrange multiplier is shown in Figure 3.

\section{Application}

\section{Hydration effects on PC lamellar multilayers}

Lamellar phases were prepared by hydrating soya lecithin (Avanti Polar), used without any further purification, using Milli-Q water in desired proportions. The density of lecithin was considered as $1,07 \mathrm{~g} / \mathrm{cm}^{3}$ and samples of various volume fractions were prepared scanning the dilution line. The homo-geneity of the samples was achieved by shaking and centrifugation processes. X-ray scattering was performed on 'powder' (randomly oriented lamellar) samples, placed in glass capillaries using the laboratory SAXS equipment (Nanostar-Bruker) placed at the Institute of Physics in the University of São Paulo. The experiments were performed at room temperature $\left(22^{\circ} \mathrm{C}\right)$. X-ray experiments in WAXS geometry have shown that for this temperature all the samples are in fluid lamellar phase, as confirmed by the broad band at $\mathrm{q}=1,5 \AA$ (not shown here). The beam diameter is about I $\mathrm{mm}$ at the sample position which leads to smearing effects that have to be taken into account in the modeling procedure (peak broadening)(Pedersen et al., I 990). In the standard SAXS setup $\left(0.01<q<0.34 \AA^{-1}\right)$, up to three Bragg reflections could be observed in the experimental scattering curves, as illustrated in figure 4 for varying li-

Barbara Bianca Gerbelli - Propriedades estruturais e elásticas de fases lamelares: O efeito da composição da membrana. 


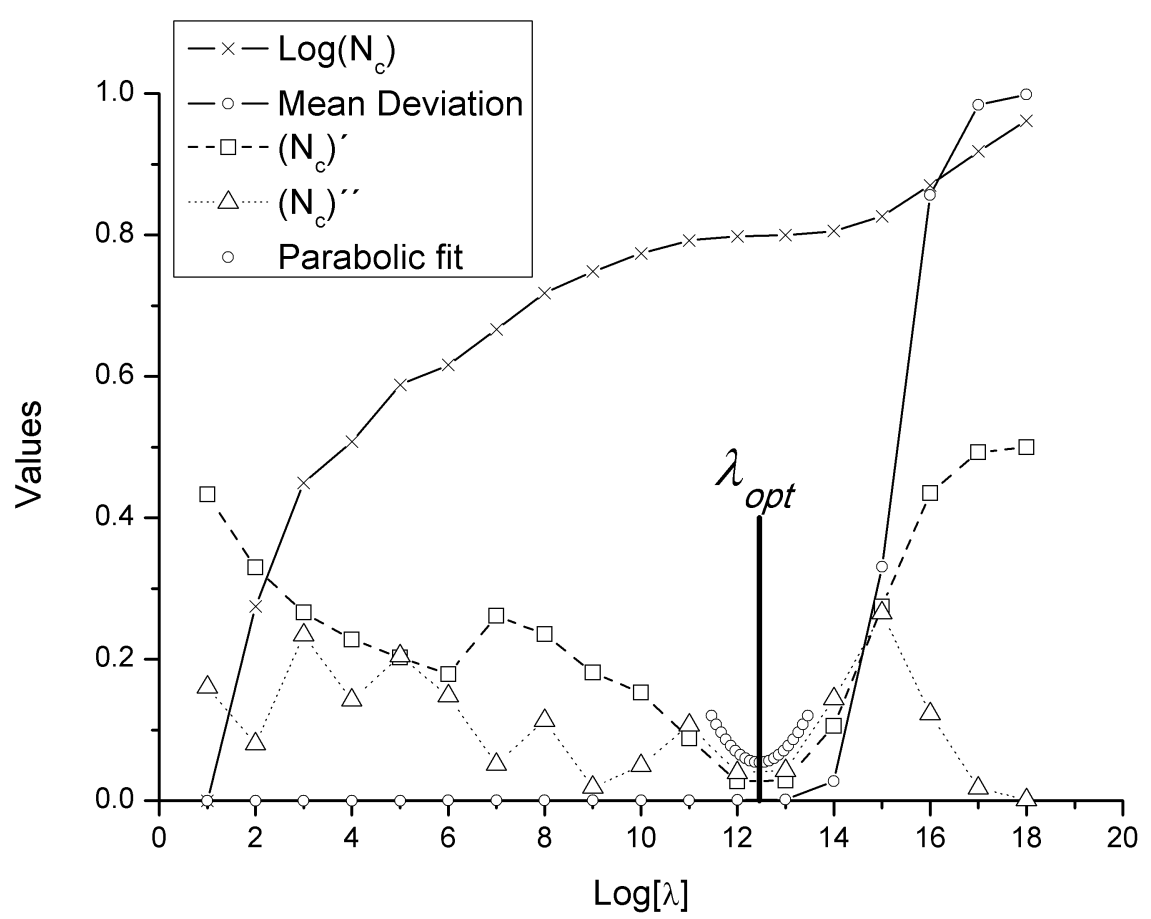

Figura D.3.: Automatic determination of the optimum Lagrange multiplier value. The information about the mean deviation value, smoothness parameter $N_{c}$ and its derivatives against $\boldsymbol{\lambda}$ are combined and adjusted by a parabolic fitting in order to provide the optimum value.

pid volume fractions $\left(\phi_{l i p}\right)$. In the figure, it can also be observed that the intensity of the second-order peak decreases with increasing lipid content and it is completely absent for the more concentrated sample: $\phi_{l i p}=\mathbf{0 . 8 7}$. This effect, however, cannot be ascribed to a decrease of smectic ordering, since the distance between the membranes is decreasing. Using the analysis method presented above (with nmax fixed and chosen equal to 4) it was possible to obtain the form and structure factors that give the best fit to the scattered intensity, shown in figure 4 as dashed and dotted lines, respectively. It was observed that the local minimum of the form factor moved in $\mathrm{q}$ space with decreasing amounts of water, which reflects structural changes in the bilayer. Therefore it is not possible to fit the set of experimental curves by the same form factor. The changes become more evident for samples with high concentrations, where the minimum of the form factor approaches the second-order peak of the structure factor.

In the diluted regime, for lipid volume fraction lower than 0,65 , the lamellar phase has attained the hydration limit, interplanar distance of approximately $62 \AA$, and coexists

Barbara Bianca Gerbelli - Propriedades estruturais e elásticas de fases lamelares: O efeito da composição da membrana. 
with water in excess. In this regime, the lamellar period and membrane thickness do not change significantly (Figure sa) when compared to the variations taking place for lipid volume fraction above $\mathbf{0 , 6 5}$. In this second regime the lamellar period decreases with dehydration, and the bilayer thickness $2 Z$ increases by approximately $6 \AA$. In the literature a smaller variation of bilayer thickness has been reported, about $2 \AA$ (McIntosh et al., I 987), however, the determination of this parameter is based on previous assumptions about the conformation of carbonic chains and polar groups to obtain the electronic contrast or from pair correlation function calculations which are not optimized during the fitting procedure (Nagle et al., I 996; Zhang et al., I 994; McIntosh et al., I 987).

The behavior of Caille parameter, $\boldsymbol{\eta}$, also indicates significant changes in membrane elasticity upon dehydration, as shown in Figure $5 b$. Three regimes can be identified; the first one, corresponding to maxi-mum hydrated lamellar phase, has a constant and high value of Caillé parameter; the second regime has an exponential decrease of the Caillé parameter with increasing lipid volume fraction. Finally, a third regime can be identified, corresponding to a strong confinement of bilayers and typical separation distances $(\mathrm{d}-2 \mathrm{Z})$ smaller than $8 \approx \AA$. For such small separation distances, the hydration force (McIntosh et al., 1987; Parsegian et al., 1979) is dominant and responsible for a strong repulsion between bilayers resulting in a constant value of Caillé parameter around $\approx 10^{-2}$.

Barbara Bianca Gerbelli - Propriedades estruturais e elásticas de fases lamelares: O efeito da composição da membrana. 
(a)

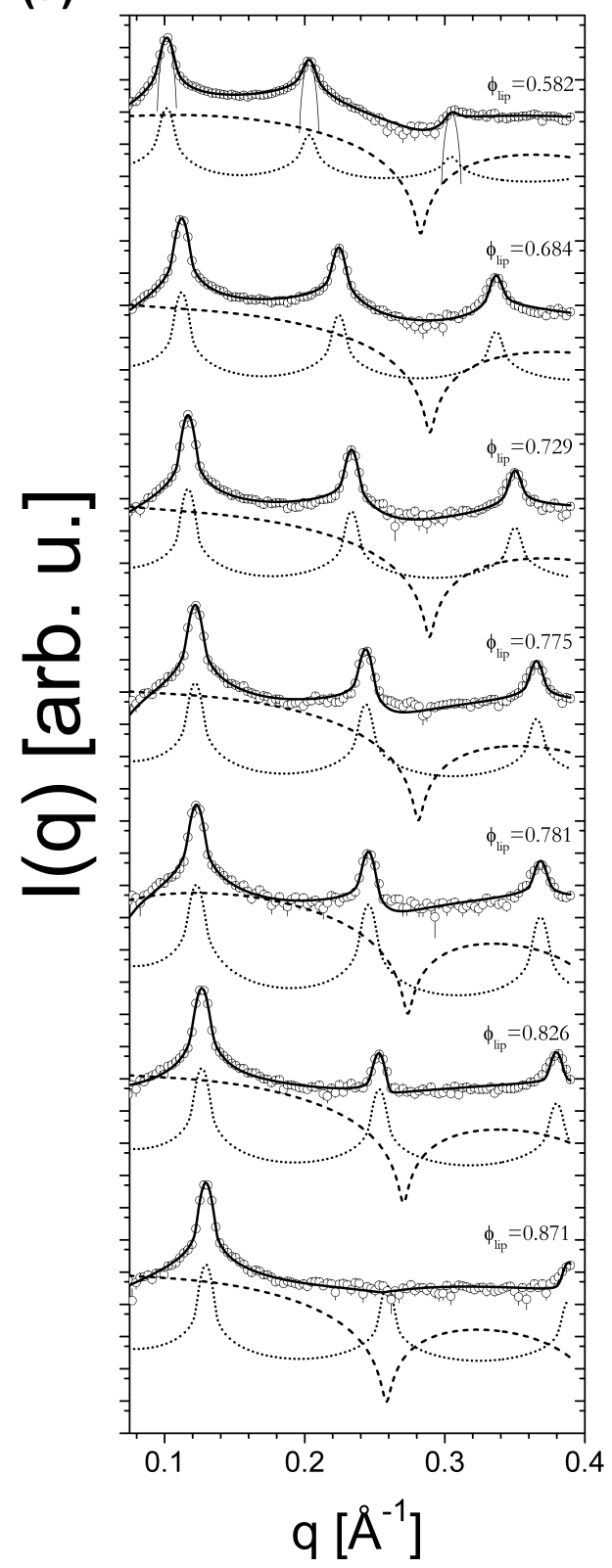

(a) (b)

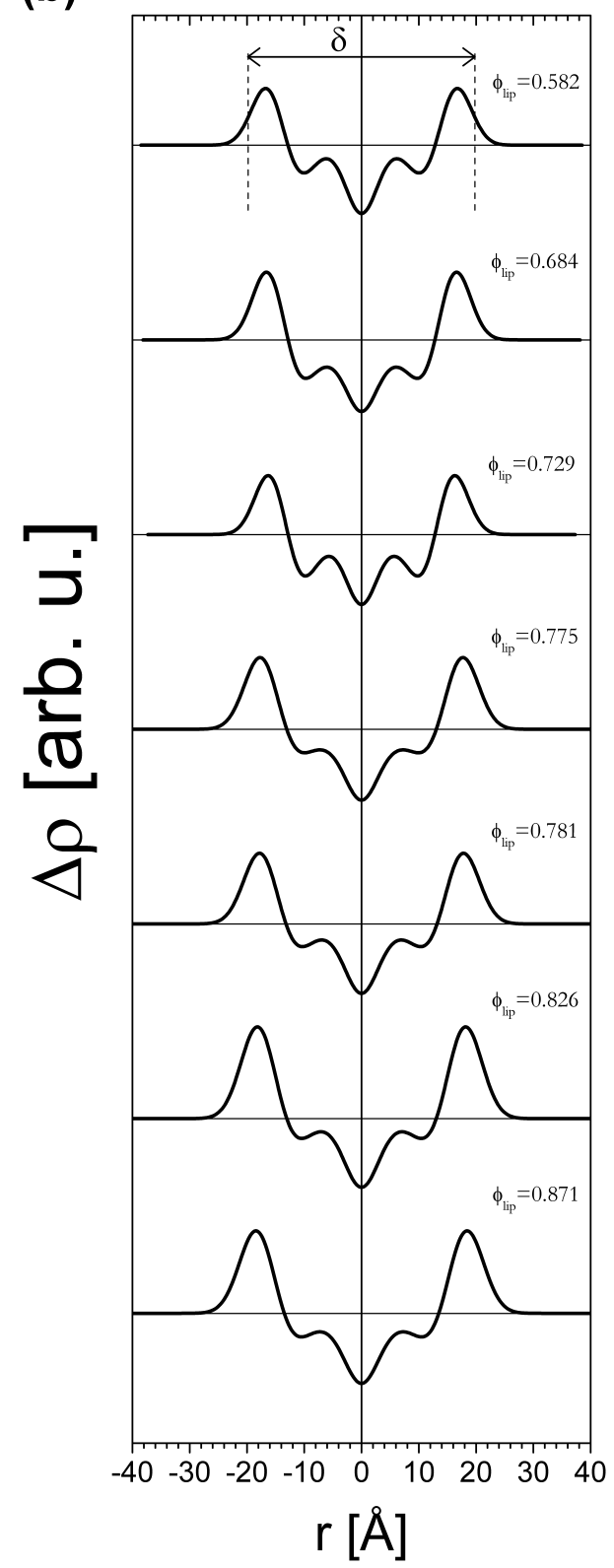

(b)

Figura D.4.: (a) Experimental scattering curves of the lamellar phase for varying lipid volume fraction $\left(\phi_{\text {lip }}\right)$. The solid thin line shown for $\phi_{\text {lip }}=\mathbf{0 . 5 8 2}$ represents the resolution functions at the three peaks positions. The dashed lines are the form factor and the dotted lines are the structure factors obtained from the minimization procedure described in the text. The solid lines represent the final fitted scattered intensity. (b) Electronic density profiles and definition of effective membrane thickness $\delta_{M}$. In all cases the curves were shifted for clarity.

Barbara Bianca Gerbelli - Propriedades estruturais e elásticas de fases lamelares: O efeito da composição da membrana. 


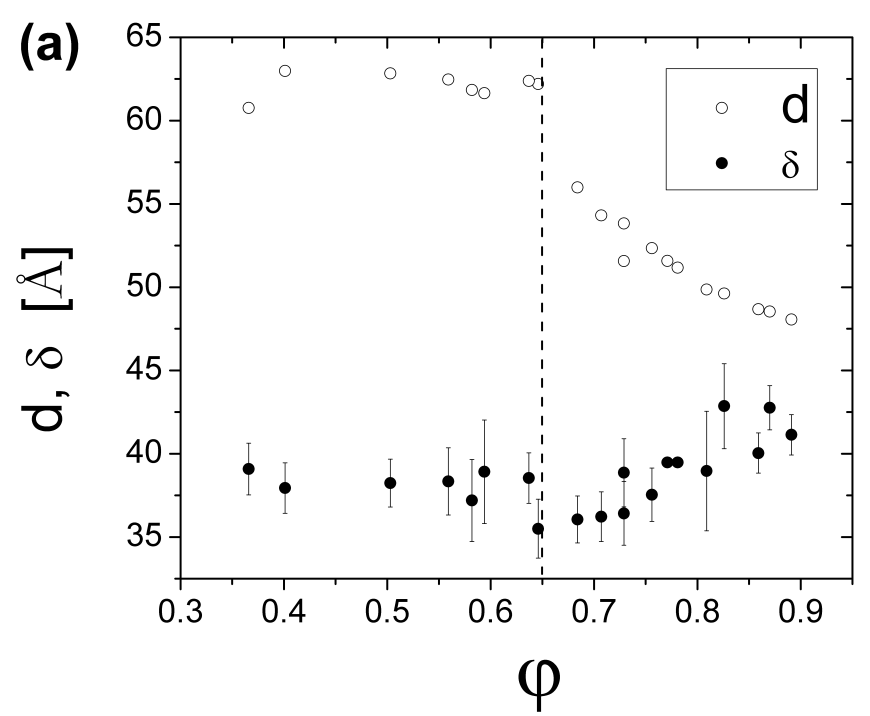

(a)

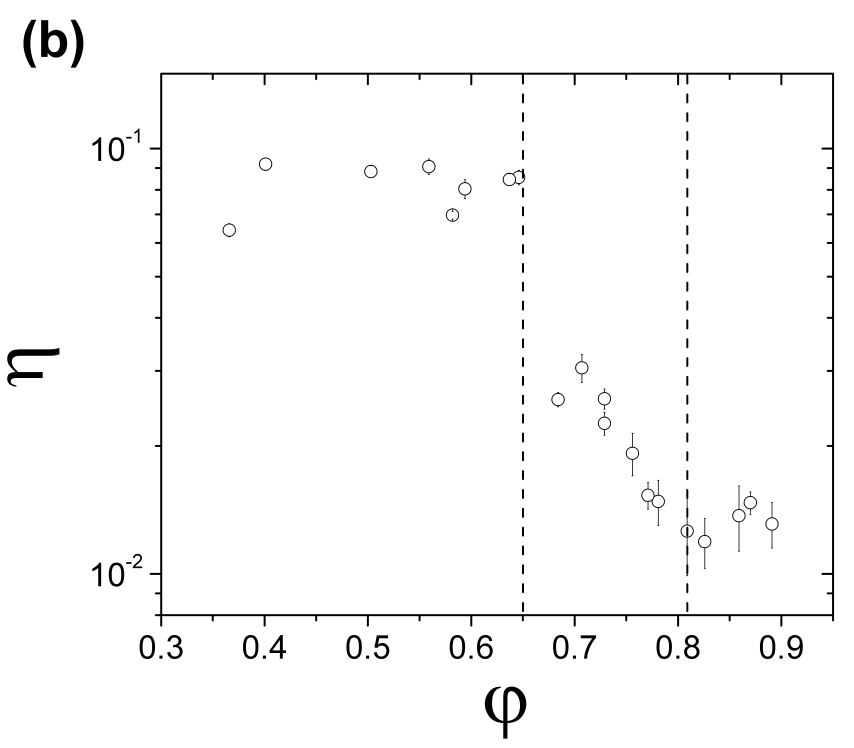

(b)

Figura D.5.: (a) Variation of lamellar period, d, and membrane effective thickness, $\boldsymbol{\delta}_{M}$, for varying lipid volume fractions. The dashed line indicates the separation of the two dilution regimes (see discussion in text). (b) Behavior of Caillé parameter. The second dashed line was added to identify the confined regime. composição da membrana. 


\section{Conclusions}

Although many studies of lecithin lamellar systems have been published in the literature, the application of Gaussian deconvolution method to fit the small-angle X-ray scattering experimental data of lecithin lamellar phase have revealed new aspects about structure and elastic properties of such system. The determination of the membrane thickness and the Caille parameter are of crucial importance for obtaining information on the interactions between the membranes. The results presented here showed that dehydration does not consist only of removing water from the space between bilayers, but that it also affects the membrane structure with consequences on its elastic properties. The change of behavior of the Caillé parameter in the confined regime occurs when water space between bilayers is reduced to a few layers of water molecules and this seems to play a major role for the elastic properties of the bilayers. The exponential dependence of the Caillé parameter on the lipid volume fraction shows that the interactions between the membranes in the dilution range are not dominated by steric interactions due to membrane fluctuations and that dipolar electrostatic interactions arising from water organization probably is also relevant.

These new aspects of lecithin lamellar system could only be obtained by the full curve fitting and simultaneous optimization of the form factor and structure factor, which is possible with the Gaussian deconvolution method. The obtained electron density profile can be subject to further analysis as, for example, specific assumptions about the membrane components (Heberle et al., 2012) which would permit the determination of the individual contributions. The new results obtained for simple lecithin systems demonstrates the robustness and potential of this modeling approach which can be applied to mono or multilayered systems. x"

\section{References}

Abramowitz, M. and Stegun, I. A. (1972). Handbook of mathematical functions : with formulas, graphs and mathematical tables. New York: Wiley.

Brotons, G., Dubois, M., Belloni, L., Grillo, I., Narayanan, T. and Zemb, T. (2005). Journal of Chemical Physics I 23.

Brzustowicz, M. R. and Brunger, A. T. (2005). Journal of Applied Crystallography 38, I26-izi.

Caillé, A. (1972). Comptes Rendus Hebdomadaires Des Séances De L Académie Des Sciences Série B 274, 891-.

Fritz, G. and Glatter, O. (2006). Journal of Physics-Condensed Matter I8, S2403-S24I9.

Glatter, O. (1977). Journal of Applied Crystallography Io, 41 5-42 I.

Barbara Bianca Gerbelli - Propriedades estruturais e elásticas de fases lamelares: O efeito da composição da membrana. 
Glatter, O. (1981). Journal of Applied Crystallography I4, IOI-Io8.

Glatter, O. and Hainisch, B. (1984). Journal of Applied Crystallography 17, 435-44I.

Glatter, O. and Kratky, O. (1982). Small Angle X-ray Scattering. Academic Press, London.

Hansen, S. (2000). Journal of Applied Crystallography 33, I4 I 5-I42 I.

Heberle, F. A., Pan, J., Standaert, R. F., Drazba, P., Kucerka, N. and Katsaras, J. (20 I 2). European Biophysics Journal 4I, 875-890.

Kotlarchyk, M. and Chen, S. H. (1983). Journal of Chemical Physics 79, 246 I-2469.

Luzzati, V. and Tardieu, A. (1974). Annual Review of Physical Chemistry 25, 79-94.

McIntosh, T. J., Magid, A. D. and Simon, S. A. (1987). Biochemistry 26, $7325-7332$.

Moore, P. B. (1980). Journal of Applied Crystallography i 3.

Nagle, J. F. and Wiener, M. C. (1989). Biophysical Journal 55, 309-313.

Nagle, J. F., Zhang, R. T., TristramNagle, S., Sun, W. J., Petrache, H. I. and Suter, R. M. (1996). Biophysical Journal 70, I419-I43I.

Nallet, F., Laversanne, R. and Roux, D. (1993). Journal De Physique Ii 3, 487-502.

Oliveira, C., Behrens, M., Pedersen, J. and al., e. (2009). Journal of Molecular Biology 387, I47-16I.

Pabst, G., Rappolt, M., Amenitsch, H. and Laggner, P. (2000a). Physical Review E 62, 4000-4009.

Pabst, G., Rappolt, M. P., Amenitsch, H. and Laggner, P. (20oob). Biophysical Journal 78, 2 IA-2IA.

Pape, E. H. and Kreutz, W. (1978). Journal of Applied Crystallography I I , 42 I-429.

Parsegian, V. A., Fuller, N. amd Rand, R. P. (1979). Proceedings of the National Academy of Sciences of the United States of America 76, $2750-2754$.

Pedersen, J. S. (1992). Journal of Applied Crystallography 25, I 29-I45.

Pedersen, J. S. (1997). Advances in Colloid and Interface Science 70, 17 7 -2 Io.

Pedersen, J. S. and Hamley, I. W. (1994a). Journal of Applied Crystallography 27, 36-49.

Pedersen, J. S. and Hamley, I. W. (1994b). Physica B I98, 16-23.

Pedersen, J. S., Posselt, D. and Mortensen, K. (1990). Journal of Applied Crystallography 23, 32 I-333.

Petrache, H. I., Gouliaev, N., Tristram-Nagle, S., Zhang, R. T., Suter, R. M. and Nagle, J. F. (1998). Physical Review E 57, 7014-7024.

Semenyuk, A. V. and Svergun, D. I. (199I). Journal of Applied Crystallography 24, 537-540.

Barbara Bianca Gerbelli - Propriedades estruturais e elásticas de fases lamelares: O efeito da composição da membrana. 
D. I Artigo publicado no Journal of Applied Crystallography (J. Appl. Cryst. (20 I 2).

Worthington, C. (1969). Biophysical Journal 9, 222-234.

Zhang, R. T., Suter, R. M. and Nagle, J. F. (1994). Physical Review E 50, 5047-5060. 



\section{REFERÊNCIAS BIBLIOGRÁFICAS}

[r] ANDERSON, R. G. W. The caveolae membrane system.

[2] MALCHAREK, A.; L., H.; HILTERHAUS; GALLA., H. Multilayer structures in lipid monolayer films containing surfactant protein c: Effects of cholesterol and pope. BIOPHYSICAL JOURNAL, v. 88, n. 4, p. 2638-2649, APR 2005.

[3] PAN, J.; TRISTAM-NAGLE, S.; NAGLE, J. Effect of cholesterol on structural and mechanical properties of membranes depends on lipid chain saturation. PHYSICAL REVIEWE, v. 80, n. 2, p. 02 I93 I, AUG 2009.

[4] A.SIDDHANTA; SHIELDS, D. Secretory vesicle budding from the trans-golgi network is mediated by phosphatidic acid levels. JOURNAL OF BIOLOGICAL CHEMISTRY, v. 273, n. 29, p. I7995-I7998, JUL I 998.

[5] HULL, M. C.; SAUER, D. B.; HOVIS, J. S. Influence of lipid chemistry on the osmotic response of cell membranes: Effect of non-bilayer forming lipids. JOURNAL OF PHYSICAL CHEMISTRY B, v. I08, n. 40, p. I 5890-I 5895, OCT 2004.

[6] SACKMANN, E. Thermo-elasticity and adhesion as regulators of cell membrane architecture and function. JOURNAL OF PHYSICAL CHEMISTRY B, v. I 8, n. 45, p. $\mathrm{R}_{785}-\mathrm{R}_{825}$, NOV 2006.

[7] WURGER, A. Bending elasticity of surfactant films: The role of the hydrophobic tails.

[8] WANG, W.; YANG, L.; HUANG, H. W. Evidence of cholesterol accumulated in high curvature regions: Implication to the curvature elastic energy for lipid mixtures.

Barbara Bianca Gerbelli - Propriedades estruturais e elásticas de fases lamelares: O efeito da composição da membrana. 
[9] TAKAHASHI, H.; SINODA, K.; HATTA, I. Effects of cholesterol on the lamellar and the inverted hexagonal phases of dielaidoylphosphatidylethanolamine. $B I O C H I$ MICA ET BIOPHYSICA ACTA-GENERAL SUBJECTS, v. I289, n. 2, p. 209-2 16, xx I 996.

[Io] SORNETTE, D.; OSTROWSKY, N. Importance of membrane fluidity on bilayer interactions. JOURNAL OF CHEMICAL PHYSICS, v. 84, n. 7, p. 4062-4067, APR I 986.

[i I] PODGORNIK, R.; PARSEGIAN, V. Thermal mechanical fluctuations of fluid membranes in confined geometries - the case of soft confinement. LANGMUIR, v. 8, n. 2, p. 557-562, FEV i 992.

[ I 2] MILNER, S.; ROUX, D. Flory theory of the unbinding transition. JOURNAL DE PHYSIQUE I, v. 2, n. 9, p. I74I-I754, SEP I 992.

[13] HELFRICH, W. Steric interaction of fluid membranes. BERICHTE DER BUNSENGESELLSCHAFT-PHYSICAL CHEMISTRY CHEMICAL PHYSICS, v. 82, n. 9, p. 927, XX 1978 .

[14] SAFINYA, C.; ROUX, D; SMITH, G.; ET AL. Steric interactions in a model multimembrane system - a synchrotron x-ray study. PHYSICAL REVIEW LETTERS, v. 57, n. 21, p. 2718-2721, NOV 1986.

[ I s] SAFINYA, C.; SIROTA, E.; ROUX, D. E. A. Universality in interacting membranes - the effect of cosurfactants on the interfacial rigidity. PHYSICAL REVIEW LETTERS, v. 62, n. Io, p. I I 34-I I 37, MAR 1989.

[ı6] NAGLE, J.; ZHANG, R.; TRISTRAMNAGLE, S.; ET AL. X-ray structure determination of fully hydrated (alpha) phase dipalmitoylphosphatidylcholine bilayers. BIOPHYSICAL JOURNAL, v. 70, n. 3, p. I4I 9-I43 I, MAR 1996.

[ 17] PABST, G.; RAPPOLT, M.; AMENITSCH, H.; ET LAGGNER, P. New evidence for gel-liquid crystalline phase coexistence in the ripple phase of phosphatidylcholines. EUROPEAN BIOPHYSICS JOURNAL WITH BIOPHYSICS LETTERS, v. 29, n. 2, p. I25-I33, Xx 2000.

[18] NALLET, F.; D., L.; ROUX, D. Modeling x-ray or neutron-scattering spectra of lyotropic lamellar phases - interplay between form and structure factors. JOURNAL DE PHYSIQUE II, v. 3, n. 4, p. 487-502, APR I 993.

Barbara Bianca Gerbelli - Propriedades estruturais e elásticas de fases lamelares: O efeito da composição da membrana. 
[19] PABST, G. Global properties of biomimetic membranes: Perspectives on molecular features. Biophysical Reviews and Letters, v. I, n. I, p. 57-84, NOV 2005.

[20] BARROSO, R. P.; RISKE, K. A.; HENRIQUES, V. B.; ET AL. Ionization and structural changes of the dmpg vesicle along its anomalous gel-fluid phase transition: A study with different lipid concentrations. LANGMUIR, v. 26, n. 17, p. 13805I 38 I 4 , SEP 2010.

[2 I] VINK, H. Precision measurements of osmotic pressure in concentrated polymer solutions. EUROPEAN POLYMER JOURNAL, v. 7, n. Iо, p. I4I I-, I 97 I.

[22] PETRACHE, H.; TRISTRAM-NAGLE, S.; GOULIAEV, N.; ET AL. Interbilayer interactions determined from high resolution x-ray scattering. BIOPHYSICAL JOURNAL, v. 74, n. 2, p. A203-A203, FEB 1998.

[23] PETRACHE, H. I.; TRISTRAM-NAGLE, S.; HARRIES, D.; KUčERKA, N.; NAGLE, J. F.; PARSEGIAN, V. A. Swelling of phospholipids by monovalent salt. JOURNAL OF LIPID RESEARCH, v. 47, n. 2, p. 302 - 309, FEB 2006.

[24] PETRACHE, H.; HARRIES, D.; PARSEGIAN, V. Alteration of lipid membrane rigidity by cholesterol and its metabolic precursors. MACROMOLECULAR SYMPOSIA, v. 2 19, p. 39 - 50, DEC 2004.

[25] ISRAELACHVILI, J. Entropic forces between amphiphilic surfaces in liquids. THE AMERICAN CHEMICAL SOCIETY, v. 20I, p. 5-, APR I99I.

[26] NELSON, D. L.; COX, M. M. Principles of biochemistry. v. FIFTH EDITION, p. -, Jan 2008 .

[27] JONES, R. A. L. Soft condensed matter. 2002.

[28] DE GENNES, P.; PROST, J. The physics of the liquid crystals. 1993.

[29] HUSSON, F.; MUSTACCHI, H.; LUZZATI, V. Entropic forces between amphiphilic surfaces in liquids. ACTA CRYSTALLOGRAPHICA, v. 1 3, n. 8, p. $668-677$, APR i 960.

[30] KARMAKAR, S.; RAGHUNATHAN, V. Structure of phospholipid-cholesterol membranes: An x-ray diffraction study. PHYSICAL REVIEW E, v. 7I, n. 6, p. 06I924, JUN 2005 .

Barbara Bianca Gerbelli - Propriedades estruturais e elásticas de fases lamelares: O efeito da composição da membrana. 
[3 I] SMITH, G.; SAFINYA, C.; ROUX, D.; CLARK, N. X-ray study of freely suspended films of a multilamellar lipid system. MOLECULAR CRYSTALS AND LIQUID CRYSTALS, v. I44, p. 235-255, I 987.

[32] SAFINYA, C. R.; SIROTA, E. B.; ROUX, D.; SMITH, G. S. Universality in interacting membranes: The effect of cosurfactants on the interfacial rigidity. PHYSICAL REVIEWLETTERS, v. 62, n. Io, p. I I 34-I I 37, MAR I 989.

[33] NINHAM, B. On progress in forces since the dlvo theory. ADVANCES IN COLLOID AND INTERFACE SCIENCE, v. 83, n. I - 3, p. I - i 7, DEC 1999.

[34] MANCIU, M.; RUCKENSTEIN, E. The polarization model for hydration/double layer interactions: the role of the electrolyte ions. ADVANCES IN COLLOID AND INTERFACE SCIENCE, v. I I 2, n. I - 3, p. I09- I 28, DEC 2004.

[35] FARAUDO, J.; BRESME, F. Origin of the short-range, strong repulsive force between ionic surfactant layers. PHYSICAL REVIEW LETTERS, v. I I 2, n. 2, p. 2I 3-2 I6, MAY 2005.

[36] GAWRISCH, K.; RUSTON, D.; ZIMMERBERG, J.; ET AL. Membrane dipole potentials, hydration forces, and the ordering of water at membrane surfaces. BIOPHYSICAL JOURNAL, v. 6i, n. 5, p. I21 3-I 223, MAY 1992.

[37] CARVELL, M.; HALL, D.; LYLE, I.; ET AL. Surfactant water interactions in lamellar phases - an equilibrium binding description of interbilayer forces. FARADAY DISCUSSIONS, v. 81, p. 223-237, MAY i 986.

[38] HELFRICH, W. Elastic properties of lipid bilayers: Theory and possible experiments. Z. Naturforsch, v. 28c, n. I, p. 693-703, JUL i 984.

[39] LIPOWSKY, R.; LEIBLER, S. Unbinding transitions of interacting membranes. PHYSICAL REVIEW LETTERS, v. 56, n. 23, p. 254I-2544, I 986.

[40] HELFRICH, W. Steric interation of fluid membrane in multilayer systems. $Z$. Naturforsch, v. 33-a, p. 305-3 i s, SEP 1977.

[4I] W. M. GELBART, A. BEN-SHAUL, D. R. Micelles, membranes, microemulsions and monolayers. Springer, I99I. Cap. 5 e 6, p. 25 I-346.

Barbara Bianca Gerbelli - Propriedades estruturais e elásticas de fases lamelares: O efeito da composição da membrana. 
[42] PODGOMIKT, R.; PARSEGIAN, V. A. Thermal-mechanical fluctuations of fluid membranes in confined geometries: The case of soft confinement. Langmuir, Washington, v. 8, p. 557-562, SEP I 991.

[43] MCINTOSH, T. J.; MAGID, A. D.; SIMON, S. A. Steric repulsion between phosphatidylcholine bilayers. Biochemistry, v. 25, p. 4948, JUN I 987.

[44] OLIVEIRA, C. L. P.; GERBELLI, B. B.; SILVA, E. R. T.; NALLET, F.; NAVAILLES, L.; OLIVEIRA, E. A.; PEDERSEN, J. S. Gaussian deconvolution: A powerful method for form-free modeling scattering data from mono and multilayered vesicles using constrained least-square fitting. Journal of Applied Crystallography, v. Accepted, 2 OI 2.

[45] ZHANG, RT; SUTER, R. N.-J. Theory of the structure factor of lipid bilayers. PHYSICAL REVIEWE, v. 50, n. 6, p. 5047-5060, DEC I 994.

[46] ROUX, D.; SAFINYA, C. R. A synchrotron x-ray study of competing undulation and electrostatic interlayer interactions in fluid multimembrane lyotropic phases. JOURNAL DE PHYSIQUE FRANCE, v. 49, p. 307-3 I 8, FEB I 988.

[47] SAFINYA, C. R.; ROUX, D.; SMITH, G. S.; SINHA, S. K.; DIMON, P.; CLARK, N. A.; BELLOCQ. Steric interactions in a model multimembrane system - a synchrotron x-ray study. PHYSICAL REVIEW LETTERS, v. 57, n. 2 I, p. 27 I 8-272 I, FEB 1986.

[48] KUCERKA, N.; LIU, Y.; CHU, N.; PETRACHEZ, H. I.; STEPHANIE TRISTRAM-NAGLE, Y.; NAGLE, J. F. Structure of fully hydrated fluid phase dmpc and dlpc lipid bilayers using x-ray scattering from oriented multilamellar arrays and from unilamellar vesicles. Biophysical Journal, v. 88, p. 2626-2637, APR 2005 .

[49] SAFINYA, C. R. Undulation forces in dilute model membrane lamellar phases. ABSTRACTS OF PAPERS OF THE AMERICAN CHEMICAL SOCIETY, v. i 98, p. 48-COLL, SEP I 989.

[5o] DA SILVA, E. R. Estrutura e dinÂmica de dna confinado entre membranas lipiidicas nẤo-catiÔnicas. 20I I. Tese (Doutorado em Física) - Universidade de São Paulo, SP, 2OII.

Barbara Bianca Gerbelli - Propriedades estruturais e elásticas de fases lamelares: O efeito da composição da membrana. 
[5 I] DE OlIVEIRA, C. L. P.; PEDERSEN, J. Program package for data treatment, analysis and modeling. ., 2009.

[52] DE OLIVEIRA, C. L. P. Estudos de espalhamento de raios $x$ a baixos Ângulos por sistemas biológicos: Teoria e aplicações. 2005. Tese (Doutorado em Física) - IFGW UNICAMP, Campinas, 2005.

[53] MCINTOSH, T.; SIMON, S. Area per molecule and distribution of water in fully hydrated dilauroylphosphatidylethanolamine bilayers. BIOCHEMISTRY, v. 25 , n. I7, p. 4948-4952, AUG i 986.

[54] MCINTOSH, T.; SIMON, S. Hydration force and bilayer deformation - a reevaluation. BIOCHEMISTRY, v. 25, n. I4, p. 4058-4066, JUL 1986.

[5s] RUBIM, R. L.; GERBELLI, B. B.; DE OLIVEIRA, E. A. Effects of fatty acids in strutural parameters of lipids membranes by saxs investigation. $20 \mathrm{I} 2$.

[56] Nallet, 2005.

[57] BAGGERJORGENSEN, H.; OLSSON, U. Experimental study of undulation forces in a nonionic lamellar phase. LANGMUIR, v. I 2, n. 17, p. 4057-4059, AUG I 996.

Barbara Bianca Gerbelli - Propriedades estruturais e elásticas de fases lamelares: O efeito da composição da membrana. 